\title{
Guided Wave Resonant Optical Structures and LED Micro Resonators for Biosensing Applications
}

\author{
Rohit Goswami \\ West Virginia University
}

Follow this and additional works at: https://researchrepository.wvu.edu/etd

\section{Recommended Citation}

Goswami, Rohit, "Guided Wave Resonant Optical Structures and LED Micro Resonators for Biosensing Applications" (2012). Graduate Theses, Dissertations, and Problem Reports. 4859.

https://researchrepository.wvu.edu/etd/4859

This Dissertation is protected by copyright and/or related rights. It has been brought to you by the The Research Repository @ WVU with permission from the rights-holder(s). You are free to use this Dissertation in any way that is permitted by the copyright and related rights legislation that applies to your use. For other uses you must obtain permission from the rights-holder(s) directly, unless additional rights are indicated by a Creative Commons license in the record and/ or on the work itself. This Dissertation has been accepted for inclusion in WVU Graduate Theses, Dissertations, and Problem Reports collection by an authorized administrator of The Research Repository @ WVU.

For more information, please contact researchrepository@mail.wvu.edu. 


\title{
Guided Wave Resonant Optical Structures and LED Micro Resonators for Biosensing Applications
}

\author{
by \\ Rohit Goswami \\ Dissertation submitted to the \\ College of Engineering and Mineral Resources \\ at West Virginia University \\ in partial fulfillment of the requirements \\ for the degree of
}

Doctor of Philosophy

in

Electrical Engineering
Lawrence A. Hornak, Ph.D., Chair
Dimitris Korakakis, Ph.D.
Jeremy Dawson, Ph.D.
Mark Jerabek, Ph.D.
Lloyd Carroll, Ph.D.

Lane Department of Computer Science and Electrical Engineering

Morgantown, West Virginia

2012

Keywords: Evanescent wave sensors, Microspheres, Optical cavity modes, RCLEDs 


\begin{abstract}
Guided Wave Resonant Optical Structures and LED Micro Resonators for Biosensing Applications

Rohit Goswami
\end{abstract}

Integrated opto-electronic and nanophotonic devices for sensing application in the fields of medicine, microbiology, environmental, safety and defense have attracted considerable attention due to their potential for achieving greater compactness, shorter response times and higher sensitivities as compared to non-optical sensing systems. Optical cavity resonant devices such as Fabry-Perot interferometers have been extensively used in lasing applications and optical sensing has been accomplished through many similar technologies.

Fiber optic and planar waveguide based resonant devices which use evanescent waves for detection of refractive index changes are one of the most widely used approaches for photonic sensors. In this work we investigate the simulations, fabrication and characterization of resonant optical cavity devices for sensing applications. Morphology Dependent Resonances (MDRs) of planar, micro-spherical and micro-cylindrical cavities were reviewed for resonance line widths, spacing between modes, and density of resonances and experimental observations of internal and external field distributions. We focus on planar thin film stacked resonant waveguide geometries, microsphere-waveguide coupled resonances, cylindrical Gallium Nitride $(\mathrm{GaN})$ microdisks for passive detection of Whispering Gallery Modes (WGMs) and electrically pumped active Resonant Cavity (RC) LED disk geometries for Vertical Cavity Modes (VCMs) as structures of interest.

Advances in stacked thin film coupled waveguide sensors enhance the selectivity and sensitivity by measuring the changes of the resonant optical modes and provide an integrated platform for label-free molecular detection. The effective surface loading detection sensitivity of the planar coupled alumina waveguide transducer was determined to be $20 \mathrm{pg} / \mathrm{mm}^{2}$ with a bulk index sensitivity of $5.6 \times 10^{-4}$ Refractive Index Units (RIU) for aqueous sucrose solutions. For circular geometry based resonators, as the physical device size approaches the wavelength of light the MDRs are enhanced by retaining longer photon 
path length times and enhancing detection due to its high $\mathrm{Q}$ factors. Circular micro-cavities not only modify the optical resonances but also distribute the resonant frequencies as compared to a planar macro-cavity. The waveguide-coupled microspheres were experimentally detected to have a minimum surface coverage limit of $0.192 \%$. Passive WGMs in GaN micro-disks showed a variation in mode spacing of $3 \mathrm{~nm}$ to $7 \mathrm{~nm}\left(\lambda^{2} / 2 \pi R n\right)$ as disk radius was reduced from $4.5 \mu \mathrm{m}$ to $2 \mu \mathrm{m}$. Micro-cylindrical Distributed Bragg Reflector (DBR) RCLEDs were designed for layer thicknesses and Multi Quantum Well (MQW) placement to enhance VCMs and LED emission output. Experimental and simulated LED spectral minima matched at $432 \mathrm{~nm}$ and $451 \mathrm{~nm}$ confirming VCMs related to $(\lambda / 2)$ cavity resonances. 


\section{Acknowledgements}

First of all, I owe my gratitude to Professor Lawrence Hornak, as he took a gamble on me. My dream of obtaining a PhD would not have been possible without his intellectual support and the physical resources he made available to me, that made my $\mathrm{PhD}$ an enriching experience. All the valuable discussions, criticisms and the training he provided will benefit me throughout my career.

I would like to thank Dr. Dimitris Korakakis for everything from learning in his classes, to group meetings and the support provided through his lab resources, which greatly helped in the successful accomplishment of this project.

I would also like to thank Dr. Jeremy Dawson, Dr. Lloyd Carroll and Dr. Mark Jerabek for participating in my committee and providing their guidance and support.

I cannot thank enough to Dr. Kolin Brown, Dr. Min Soo Lim, Dr. Sridhar Kuchibhatla and Bashar Hamza for all the technical discussions related to my academic research.

I have also received great help from all of my lab mates and the people in the LED research group. I will not attempt to list their names for fear of leaving someone out. You all know how important you are to me.

Thanks to all of my friends for making my life a lot more balanced in addition to academic research. I thank Danielle Brady for supporting me when I needed encouragement. Finally, I thank my family for their constant love and support.

I dedicate this dissertation in loving memory of my grandparents. 


\section{Index of Contents}

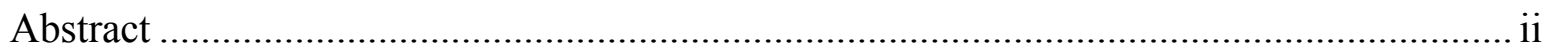

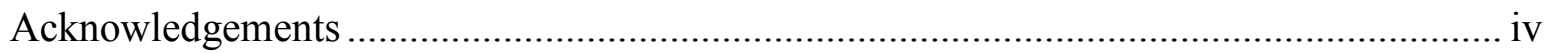

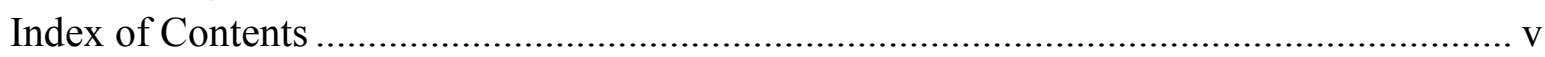

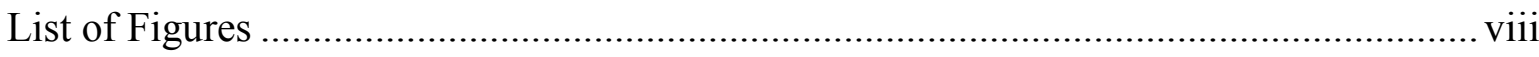

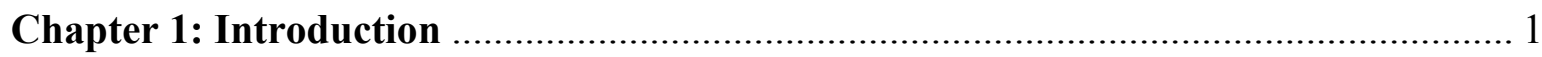

1-1 Background of Optical Biosensors .............................................................. 1

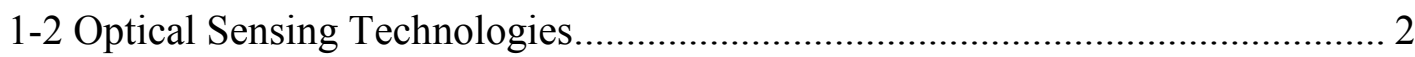

1-2-1 Fluorescent Biosensors ................................................................... 2

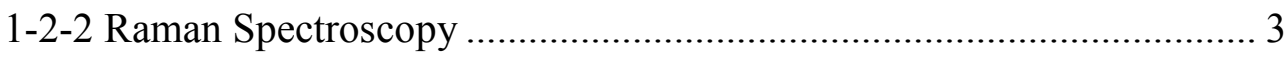

1-2-3 Surface Plasmon Resonance .............................................................. 5

1-2-4 Resonant Planar Waveguide Sensors …………................................. 6

1-3 Resonant Micro Cavity Sensors................................................................... 7

1-3-1 Planar Waveguide Coupled Resonators: The SPARROW Device ...... 8

1-3-2 Whispering Gallery Mode Devices ..................................................... 10

1-3-3 LED Resonant Devices.................................................................. 10

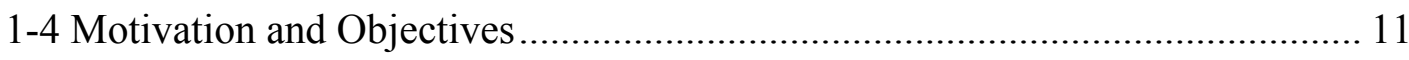

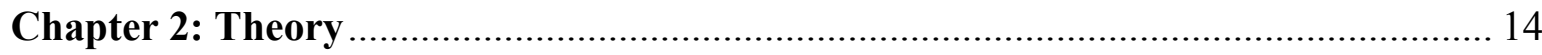

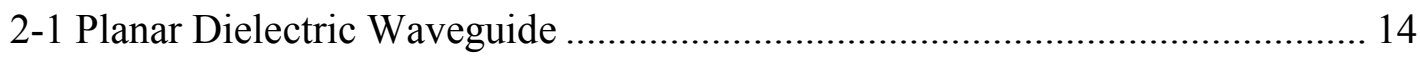

2-1-1 Maxwell's Equations and Electromagnetic Analysis.......................... 14

2-1-1-1 Maxwell's Equations in Integral Form ................................. 14

2-1-1-2 Maxwell's Equations in Differential Form........................... 16

2-1-1-3 Maxwell's Equations for Harmonic Fields ........................... 17

2-1-1-4 Helmholtz Equation.......................................................... 18

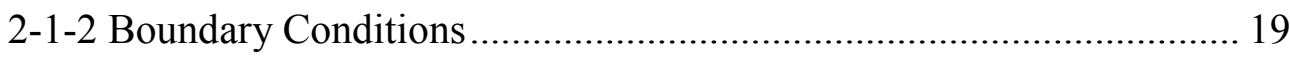

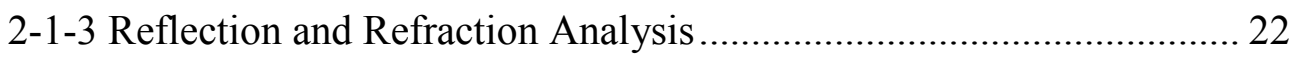

2-1-4 Goos-Hanchen Effect and Evanescent Waves.................................... 24

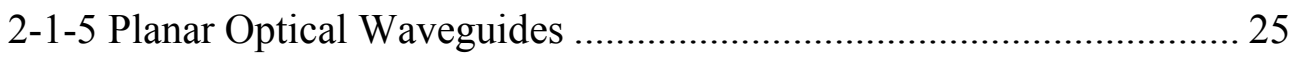

2-1-5-1 Basic Waveguide Equations.................................................. 26

2-1-6 Scattering/Radiation Losses in a Planar Waveguide ........................... 29

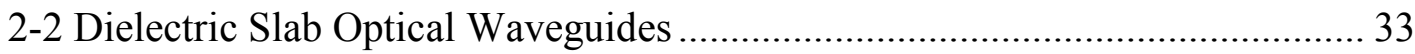

2-2-1 Three Layer Step Index Dielectric Slab Waveguide .......................... 33

2-2-2 Guided Modes of the Symmetric Waveguide...................................... 35

2-2-2-1 Transverse Electric (TE) Guided Modes................................. 35

2-2-2-2 Transverse Magnetic (TM) Guided Modes .......................... 37 


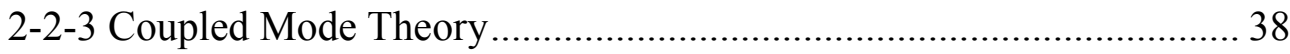

2-2-4 Sensitivity Evaluation for TE modes................................................... 41

2-2-5 Resonant Waveguide Structure as Biosensors.................................... 42

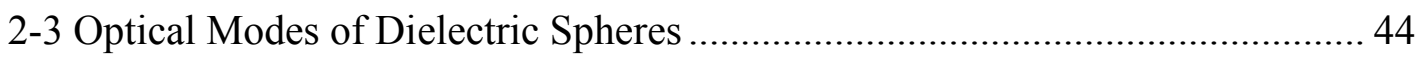

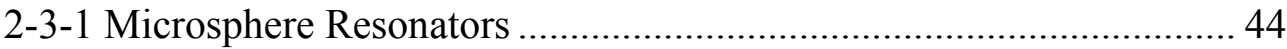

2-3-2 Loss Mechanisms (Intrinsic and Extrinsic) ......................................... 47

2-3-2-1 Microsphere Intrinsic Scattering and Absorption Losses ... 47

2-3-2-2 Microsphere Surface Radiation Losses ................................. 49

2-3-3 TE Modal Solutions......................................................................... 51

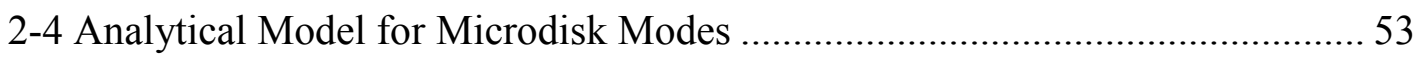

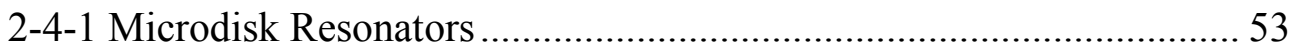

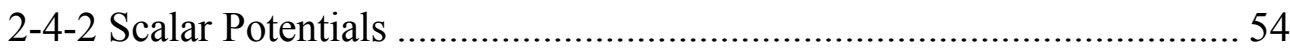

2-4-3 Microdisk Whispering Gallery Modes ………………..................... 56

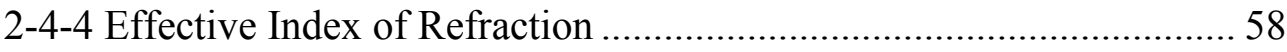

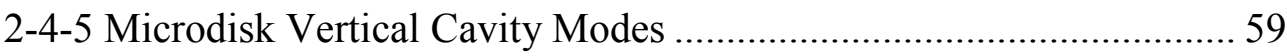

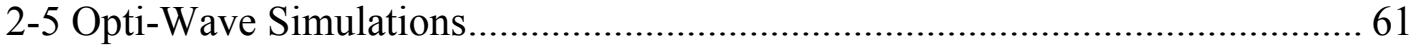

2-5-1 Microcavity Resonators as Passive Components ................................ 61

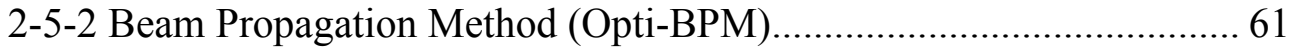

2-5-3 Finite Difference Time Domain Method (Opti-FDTD) ....................... 64

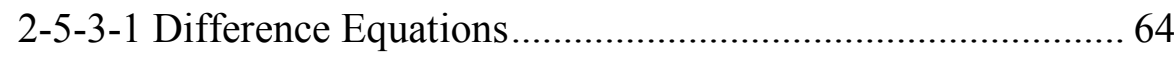

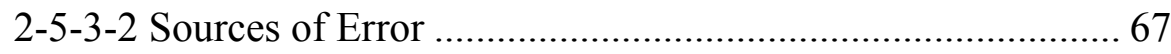

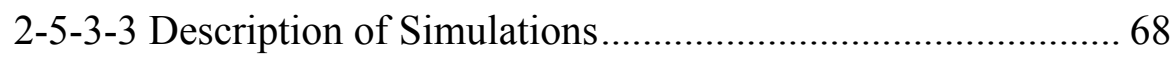

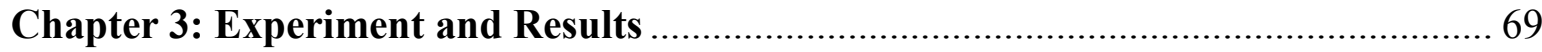

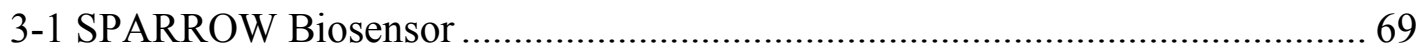

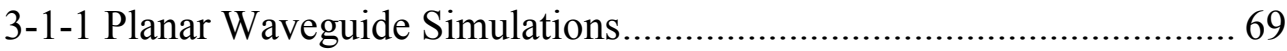

3-1-2 Coupled Waveguide (SPARROW) Simulations ................................. 71

3-1-3 Biolayer Design and Simulations ………………………………......... 75

3-1-4 Optical Characterization of Alumina Waveguides .............................. 78

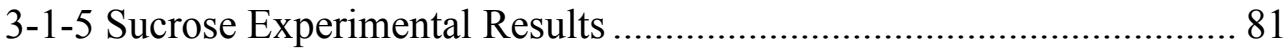

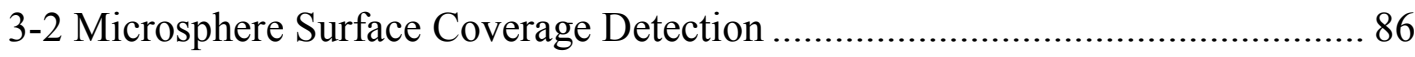

3-2-1 Microsphere-Waveguide Coupled System Simulations ...................... 87

3-2-2 Scattering Loss Analysis Simulations ............................................. 91

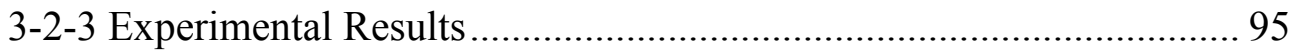

3-2-3-1 Fluorescent Experiments ................................................... 95

3-2-3-2 Prism Coupling Experiments ............................................. 99

3-3 Whispering Gallery Modes Detection in GaN Disks...................................... 100

3-3-1 Passive GaN Disk Simulations .......................................................... 101 
3-3-2 Fabrication of GaN Disk Pattern .................................................... 108

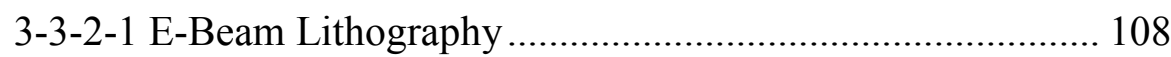

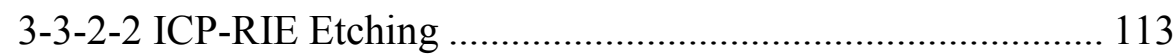

3-3-3 Optical Characterization of Whispering Gallery Modes ................... 118

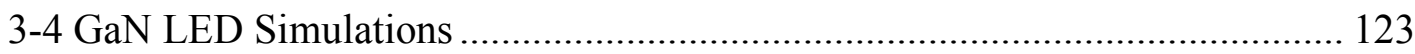

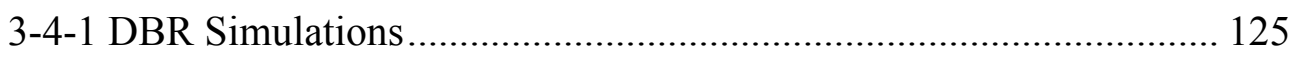

3-4-2 Passive LED Reflectivity Simulations ............................................ 128

3-4-3 Vertical Cavity Modes and RCLEDs Simulations ........................... 129

3-4-4 LED Electroluminescence Simulations ........................................... 135

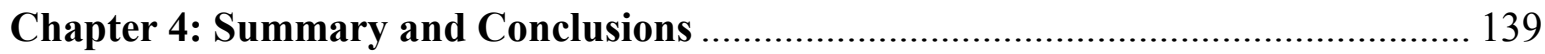

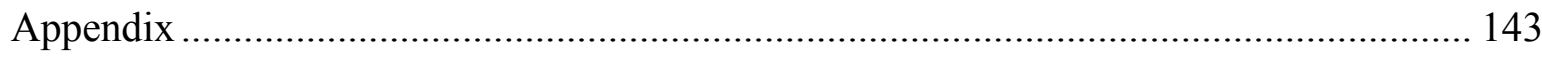

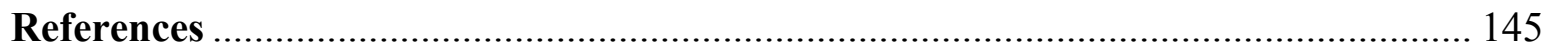




\section{List of Figures}

Fig 1 Energy Band Diagram for Fluorescence and Phosphorescence [6]........................ 3

Fig 2 Anti-Stokes, Rayleigh and Stokes Scattering Energy Diagram [7, 8] ................... 4

Fig 3 Surface Plasmon Sensor Architecture and Detection of Resonance Shift................ 5

Fig 4 Planar Waveguide Based Optical Sensor........................................................... 7

Fig 5 Resonance Peak Shift with Molecular Absorption on a Circular Micro-resonator .... 8

Fig 6 SPARROW Device Architecture ..................................................................... 9

Fig 7 Dielectric-dielectric boundary across two media ............................................... 19

Fig 8 The Refraction of displacement vector $\mathrm{D}$ at a dielectric-dielectric boundary .......... 21

Fig 9 Reflected and Refractive Wave at a Boundary Interface ................................... 23

Fig 10 Goos-Hanchen Displacement at the interface of two medium ............................ 24

Fig 11 A Three-Layer Dielectric Slab Waveguide ..................................................... 34

Fig 12 Electric Field Distribution of different modes in a Symmetric Slab Waveguide .... 35

Fig 13 TE Mode Patterns of a Symmetric Waveguide................................................. 37

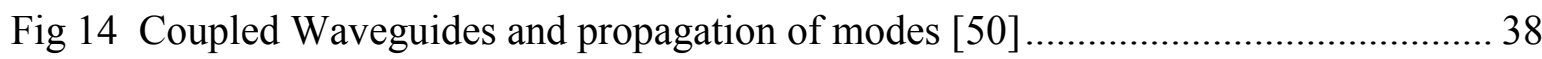

Fig 15 Schematic representation of slab waveguide sensor ..................................... 41

Fig 16 Evanescent wave $(50 \mathrm{~nm})$ interaction with analyte in microchannel $(1200 \mu \mathrm{m}) \ldots . .43$

Fig 17 Whispering gallery mode in a microsphere ..................................................... 45

Fig 18 Evanescent coupling from a planar waveguide to microsphere modes ................. 46

Fig 19 Whispering Gallery Modes in a Microdisk with different radial mode numbers... 54

Fig 20 Geometry and Coordinates of Microdisk ...................................................... 57

Fig 21 Standing wave pattern formed in an optical cavity .......................................... 59

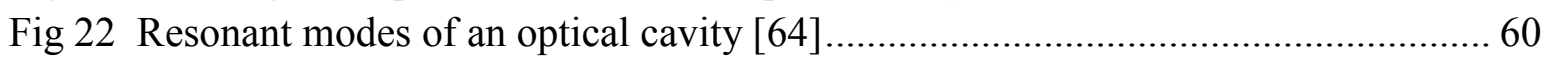

Fig 23 Optical path divided into a series of lenses in Opti-BPM software [66] ................ 62

Fig 24 Ex and Hy defined in a half-integer staggered grid [68] ....................................6 65

Fig 25 Yee Cell showing staggered grid in three dimensions [69] ................................. 66

Fig 26 Single TE Mode (Ey field) for Symmetric and Asymmetric Waveguides ............. 70

Fig 27 TE Mode (Ey field) for Asymmetric Waveguides with Different Thicknesses ..... 70

Fig 28 Designed Coupled Waveguide for Water Superstrate ........................................ 71

Fig 29 Optical Intensity for Coupled Waveguides..................................................... 72

Fig 30 Optical Mode Profile in Un-Coupled Waveguides (Air Superstrate) ..................... 73

Fig 31 Coupling Length and the even mode 'TE0' and second odd mode 'TE1' [72]...... 73

Fig 32 Even Mode (TE0) and Odd Mode (TE1) in Coupled Waveguides ....................... 74

Fig 33 Index Variations of Biolayer Superstrate and Coupled Power ............................ 76

Fig 34 Power in Bottom Waveguide with Changes in Thickness and Index of Top, Bottom

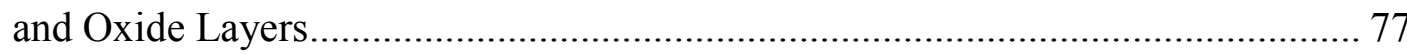

Fig 35 (a) Metricon 2010 Prism Coupler Setup, with Aligned Waveguide Sample and CCD 
Camera (b) Ray Diagram Showing Coupled Optical Path.................................. 78

Fig 36 Laser Beam and CCD Camera Noise Characterization ....................................... 79

Fig 37 Propagated Laser Power in Alumina Waveguide ............................................... 80

Fig 38 Optical Intensity Decay and MATLAB Image of Waveguide Streak ................... 80

Fig 39 Transducer Structure with a Prism, PDMS microchannel and CCD Camera......... 81

Fig 40 PDMS microfluidic channel fabrication steps and channel sidewall profile.......... 83

Fig 41 (a) Sample flow setup with power scattered from the top waveguide at PDMS-air interface and propagated power in the bottom waveguide and (b) Power drop in a single waveguide with application of SU-8 layer .............................................. 84

Fig 42 Sucrose Solution Loading vs. Optical Power Change for Three Consecutive Flows and OptiBPM Simulated Transducer Response. .................................................. 85

Fig 43 WGMs in an Optically Coupled Microsphere-Waveguide System ...................... 87

Fig 44 Distorted WGMs at an Off Resonance Wavelength .......................................... 88

Fig 45 Optical Power Loss in WG vs. Microsphere Radius (Left) and Coupled Power in the Microsphere vs. distance from WG (Right) ..................................................... 88

Fig 46 Microsphere Coupled Power (Top) and WG Output Power (Bottom) vs. Coupling Distance and Differential plots (Right) ......................................................... 89

Fig 47 WGMs as Spectral Minima in WG Transmission (Blue) and WGM Peaks inside Microsphere Cavity (Green) ......................................................................... 90

Fig 48 On-Resonance and Off-Resonance when the microsphere radius is increased by 35

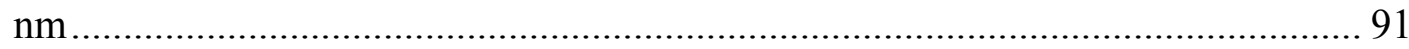

Fig 49 Shift of resonance peaks with the radius change of the microsphere .................... 91

Fig 50 Microsphere Internal Optical Losses vs. Refractive Index .................................. 92

Fig 51 Microsphere External Optical Losses vs. Surface Roughness.............................. 93

Fig 52 Microsphere External Scattering Losses vs. Refractive Index at Various Radii ..... 94

Fig 53 Microsphere Internal and External Losses vs. Refractive Index at Various Surface

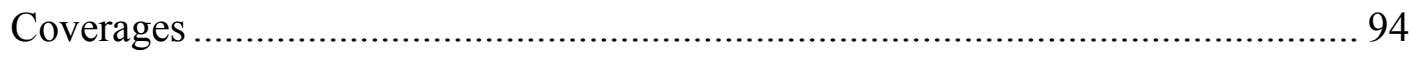

Fig 54 Microsphere External Losses vs. Microsphere Surface Coverages ....................... 95

Fig 55 Microsphere Binding on Waveguide Surface (left) and MATLAB Processed Image (right) with Surface Coverage $4.4263 \%$

Fig 56 Microsphere Binding on Waveguide Surface (left) and MATLAB Processed Image (right) with Surface Coverage $4.268 \%$

Fig 57 Microsphere Binding on Waveguide Surface (left) and MATLAB Processed Image (right) with Surface Coverage $4.0983 \%$ 98

Fig 58 Real Time Measurements for the Microsphere Surface Coverage Binding on Alumina for Repetitive Flows 98

Fig 59 Optical Power Drop in the Alumina Waveguide due to Microsphere Binding ....... 99

Fig 60 Simulated Microsphere Scattering Losses and Experimental Surface Coverage and 


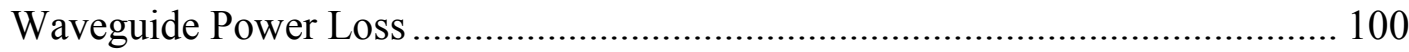

Fig 61 MATLAB Simulated Gaussian Modulated Wave-Packet Pulse ……………....... 102

Fig 62 Internally Excited GaN Disk with a Single Point Source at the Center ................ 103

Fig $63 \mathrm{GaN}$ Microdisk with Point Sources in Red and Observation Points and Planes in

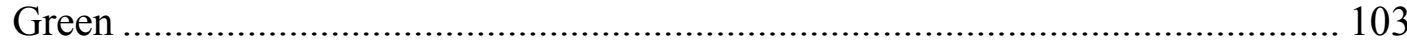

Fig 64 Opti-FDTD Simulations of GaN Disk with Multiple WGMs ................................ 104

Fig 65 Resonant Wavelength Peaks inside the Microdisk Cavity ..................................... 104

Fig 66 Guided-wave Optical Modes in a Microring Structure........................................... 105

Fig 67 Excitation of First order WGMs (Left) and Side View of Vertical Mode (Right) 105

Fig 68 Separation of Whispering Gallery, Radial and Vertical Cavity Modes.................. 106

Fig 69 GaN Micro-disk Structure on a GaN Substrate _................................................. 106

Fig 70 Asymmetric WGM Profile due to Nonlinear Effective Index (Substrate thickness = $1 \mu \mathrm{m})$

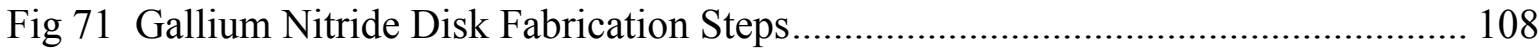

Fig 72 Scanning Electron Microscope Components [85] ................................................. 110

Fig 73 Developed PMMA Holes Pattern on GaN sample (Dose: $400 \mu \mathrm{C} / \mathrm{cm} 2$ )................ 111

Fig 74 Developed PMMA Holes Pattern on GaN sample (Tilted View).......................... 112

Fig 75 Nickel Disks as Hard Mask on GaN sample (After PMMA Lift off)..................... 113

Fig 76 A Basic Plasma Etch System Schematic [86]....................................................... 114

Fig 77 GaN Disk Pattern at a Higher DC Bias Voltage of -150 V Showing Ion Damage to

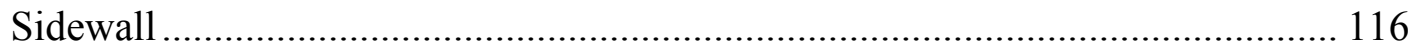

Fig 78756 nm Thick GaN Disk with Damaged Sidewall ............................................. 116

Fig 79 GaN Disks Sidewall Quality Improvement with Lower DC Bias ......................... 117

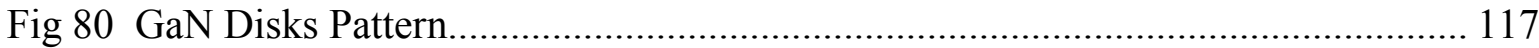

Fig 81 Side View and Top View of GaN Disks Pattern................................................ 118

Fig 82 Optical Mode Propagation in GaN Disk $(800 \mathrm{~nm})$ Simulated as a Ridge Waveguide

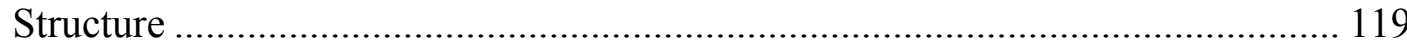

Fig 83 Optical Setup for WGM Coupling in GaN Disk Pattern ....................................... 119

Fig 84 Whispering Gallery Modes as Spectral Minima for 4.5 mm Radius Disk ............. 120

Fig 85 Whispering Gallery Modes as Spectral Minima for 3.5 m Radius Disk ............. 121

Fig 86 Whispering Gallery Modes as Spectral Minima for $2 \mu \mathrm{m}$ Radius Disk ................ 121

Fig 87 Matched Experimental and Simulated WGM Spectra for $3.5 \mu$ m Radius Disk .... 122

Fig 88 Matched Experimental and Simulated WGM Spectra for $2 \mu \mathrm{m}$ Radius Disk ....... 122

Fig 89 Gallium Nitride LED Structure [84] ................................................................ 124

Fig 9012 Period Digital Alloy DBR Structure on Sapphire Substrate [84] ..................... 125

Fig 91 Electromagnetic Intensities for 6, 12, 18 and 25 Period DBR Reflectivity........... 126

Fig 92 Experimental [88] and Simulated DBR Reflectivities for 6, 12 and 18 Periods.....127

Fig 93 Experimental [88] and Simulated LED Reflectivities on 6, 12 and 18 Period DBR 
structures 128

Fig 94 Side View of the Vertical Cavity Modes for a Longer and Shorter LED Cavity .. 130

Fig 95 Top (WGM) and Side (VCM) View in LED Cavity (Disk Thickness $=2.15 \mu \mathrm{m}) 130$

Fig 96 Mode Profile Shift and Reduction in Substrate Power Loss with a DBR LED.... 131

Fig 97 Mode Profile shift and Higher Modes for Longer LED Cavities ........................ 131

Fig 98 RCLED Cavity Length and Placement of Quantum Well on Anti-node............... 132

Fig 99 RCLED Optical Intensity as a Function of Cavity Length ................................. 133

Fig 100 LED and Designed RCLED Structures (Showing Refractive Indices) and Optical

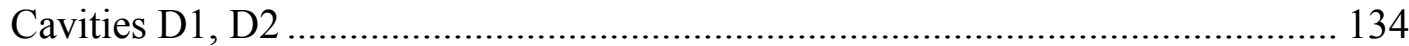

Fig 101 Simulated Intensity (Left) and Spectra (Right) for LED and Designed RCLED . 134

Fig 102 Simulated LED Structure and Optical Intensities in Different Regions of LED .. 136 Fig 103 Simulated and Experimental [88] LED EL Plots at 4V (Left) and 4.5 V (Right) for No DBR, 6, 12 and 18 Period DBR LEDs ................................................... 137 


\section{Chapter 1}

\section{Introduction}

Optical sensors that utilize resonant modes present in planar coupled waveguides and in a looped or circular waveguide structures are reviewed in this chapter. This provides a background for the present study that analyzes the sensitivity to surface refractive index changes obtainable from specific planar and circular geometries of optical resonant structures.

\section{1-1 Background of Optical Biosensors}

Optical biosensors are known for their high sensitivity and non destructive analyte analysis and detection. Optical sensing is generally based on planar waveguides, surface plasmon resonance, Raman scattering, photonic crystals, optical fibers and resonant cavity technologies. The working principle is mostly based on adsorption of analyte species and changes in optical properties of the surroundings are measured by the transducer. It is a powerful detection tool with tremendous applications in environmental, drug analysis, homeland security and medicine [1].

The two main detection methodologies used are fluorescence based detection and label free detection. The fluorescence based detection uses a labeled fluorescent tag attached to the analyte molecules and fluorescence indicates the presence of target molecules. The drawback in this process is that labeling can alter the optical properties of target molecules and the quantitative analysis is challenging. On the other hand, a label free

process is much better controlled and easy for qualitative and quantitative analysis. The molecules are detected in their original form without any specific labeling. The adsorption of target molecules on sensor surface produces a refractive index change which is proportional to the output optical signal.

The main factors influencing the performance of a biosensor are the quality of 
transduction signal, microfluidic design, sensitivity and selectivity of target analyte and immobilization chemistry. In optical biosensors, sensitivity and selectivity are important parameters which evaluate the sensor performance and efficiency. The sensitivity and selectivity of a sensor are dependent on the limit of detection of the transducer signal and a specific response to target analyte binding. Physically, the sensitivity is a measure of the smallest light-matter interaction which can produce a signal higher than the system noise [24]. The best way to make a biosensor more sensitive is to increase the light matter interaction, which in most cases is to increase the fraction of evanescent waves interacting with the target.

In the past decade, optical sensing has been a fast growing area and various optical structures have been used for label free detection. A number of detection methods employing label free optical detection have been reported in the literature which includes Raman spectroscopy, interferometers, surface plasmon resonance, optical waveguide and optical cavity resonance based biosensors.

\section{1-2 Optical Sensing Technologies}

\section{1-2-1 Fluorescent Biosensors}

Fluorescence is the phenomena in which electromagnetic radiation is first absorbed and then emitted by a substance and generally the emitted radiation has a lower energy as compared to the absorbed light. The incident radiation excites the molecules to a higher energy state which depends on the incident wavelength and the relaxation process emits radiation which creates the fluorescence effect. The difference in incident and emitted radiation depends on the optical properties of the molecule or dyes which is used as a tool for biosensing applications. The photosensitive dyes are attached to selective target molecules and fluorescent spectra are studied for molecular detection. The fluorescence process is characterized by its lifetime, which is the excited time of the molecule and is of 
the order of few Pico-seconds to Femto-seconds. Fluorescent optical biosensors are widely used for cell and tissue analysis as well as DNA sequencing [5].

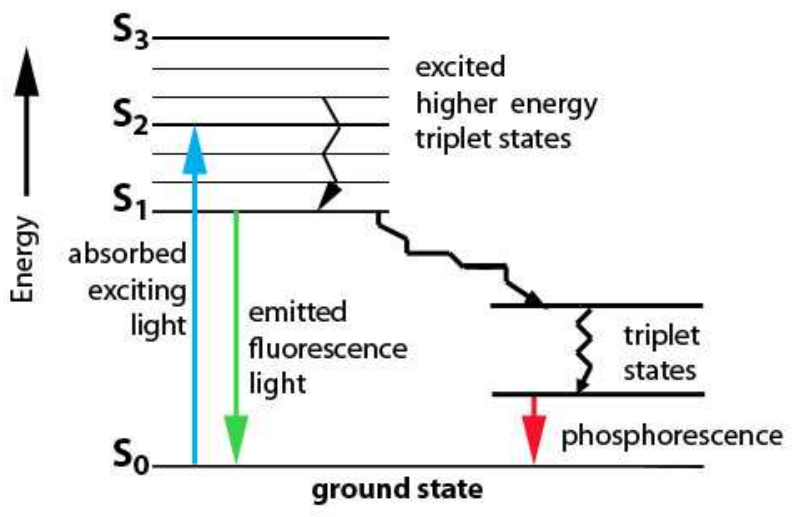

Fig 1 Energy Band Diagram for Fluorescence and Phosphorescence [6]

\section{1-2-2 Raman Spectroscopy}

When an electromagnetic wave interacts with a molecule, the electrons in the constituent molecules are perturbed periodically at the excitation frequency $(v)$ of the incident wave. This perturbation in the molecular electron cloud leads to oscillations and vibrations which create an Induced Dipole Moment. These dipole moment vibrations exist for a constant time and result in emission of scattered light. The collision process of electromagnetic waves with matter is considered to be elastic or inelastic. The majority of photons colliding with the molecule have no change in energy, which is known as Elastic Scattering (Rayleigh scattering), hence leading to an identical emitted wavelength as the incident beam. The Rayleigh scattering of sunlight in the atmosphere is the main reason for blue color of the sky. A small number of photons either lose or gain energy after colliding with the molecule and this is known as Inelastic Scattering. This is also known as Raman Scattering, and the wavelength of scattered light is dependent on the interaction of the incident EM wave and the molecular structure. 


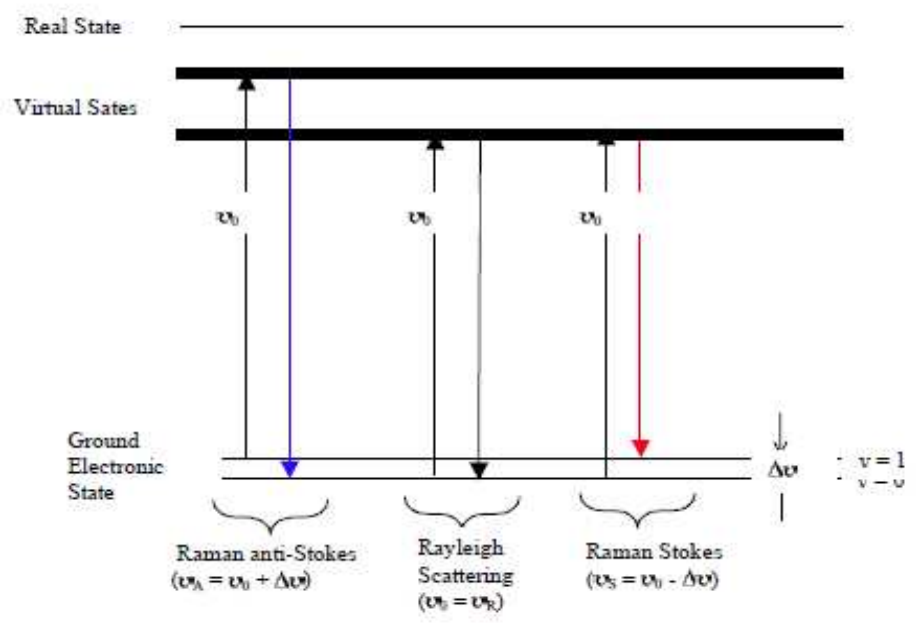

Fig 2 Anti-Stokes, Rayleigh and Stokes Scattering Energy Diagram [7, 8]

In Raman scattering, the energies of incident and scattered photons are different, which is explained by the energy band diagram of the absorption and scattering process. The energy diagram shows the energy states for Anti-Stokes, Rayleigh and Stokes scattering. When the photon reflects with the same energy as the incident photon, then it corresponds to Rayleigh scattering. The anti-stokes scattering happens when photon gains energy after collision and hence the scattered photon has a higher energy and shorter wavelength. When photon energy is lost to the rotations and vibrations of the molecule, the scattered photon has lower energy and longer wavelength, which is stokes scattering.

The anti-stokes scattering is dependent on the number of molecules in the excited Vibrational states, which gives rise to anti-stokes peaks in the spectrum. Generally, at room temperature more molecules exist in the ground Vibrational states than the excited states. Hence, more and higher intensity peaks are observed in the Stokes band rather than the antistokes band [7, 8]. Raman spectra of a molecule can give significant information about the molecular structure, and the peak intensities can be characterized for quantitative analysis. 


\section{1-2-3 Surface Plasmon Resonance}

Surface plasmons are oscillations of "free electron gas" at optical frequencies which are confined at the interface between a metal film and dielectric. Due to total internal reflection of polarized monochromatic light in the high index dielectric (prism), the evanescent field extends across the metal film, which interacts with free oscillating electrons [9]. Energy from the incident light is lost to plasmons, resulting in spectral minima observed at the reflected output at an acutely defined resonant angle, dependent on the index of the analyte medium close to the metal surface. Adsorption of biomolecules on the metal film changes the index of the analyte medium (within evanescent field region) and produces a shift of the resonance angle known as the SPR shift [10].
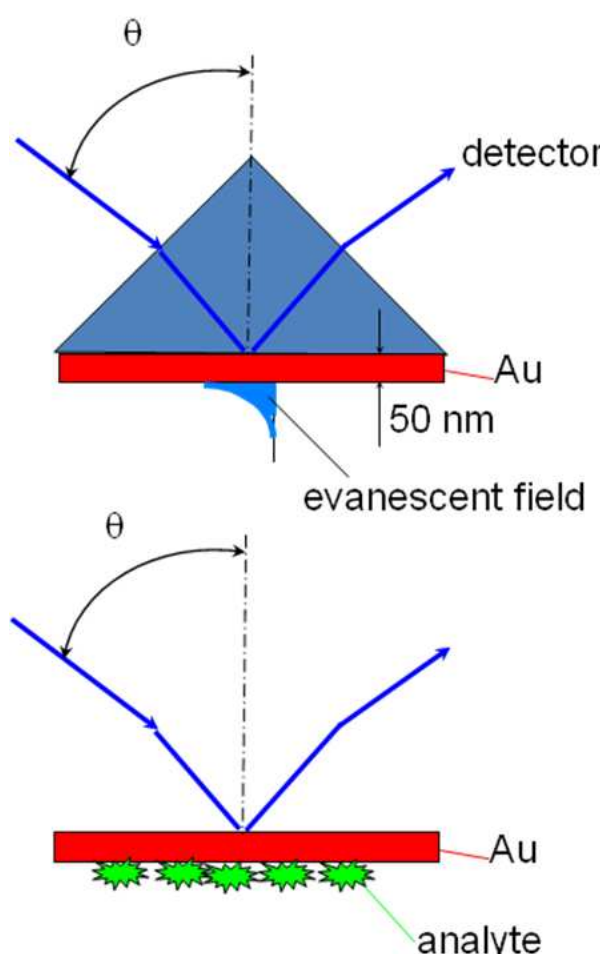
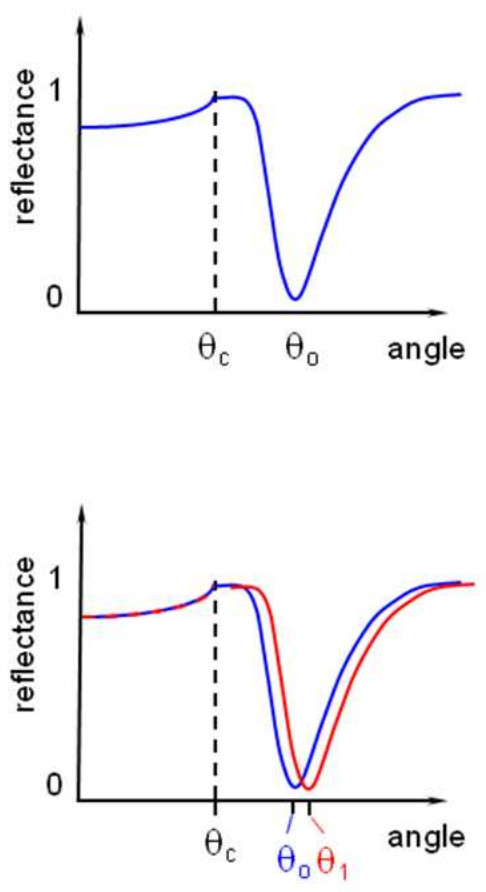

Fig 3 Surface Plasmon Sensor Architecture and Detection of Resonance Shift

The basic instrumentation and working principle of the SPR sensor is as follows. When polarized light is incident through a prism on a sensor chip with a thin metal film on top, the light is totally internally reflected by the prism-metal interface. On changing the angle of incidence, and monitoring the intensity of the reflected light, the intensity of the 
reflected light passes through a minimum. At this angle of incidence, the light will excite surface plasmons, inducing surface plasmon resonance, causing a dip in the intensity of the reflected light. Photons of p-polarized light can interact with the free electrons of the metal layer, inducing a wave-like oscillation of the free electrons and thereby reducing the reflected light intensity. The angle at which the maximum loss of the reflected light intensity occurs is called resonance angle or SPR angle. The SPR angle is dependent on the optical characteristics of the system, e.g. on the refractive indices of the media at both sides of the metal, which is usually gold. The refractive index on the prism side is constant and the index of analyte medium adsorbed on the metal surface (within evanescent wave penetration depth) is detected as a change in the SPR angle, and provides information on the kinetics of biomolecular adsorption on the sensor surface [11-13].

A method for enhancing the SPR response sensitivity is to use sensor surfaces that would amplify the change in refractive index properties of the sensor surface-solution interface. Mass labels such as latex nanoparticles/nanospheres have been linked to analytes. The beads amplify the change in refractive index when antibodies bind to the immobilized antigen layer on the sensor surface. This leads to a larger shift in resonance angle or wavelength. Although SPR technology demonstrates high sensitivity for a variety of applications, its lack of portability hinders use in field situations. The component alignment necessary to accurately determine resonance angle/wavelength shifts precludes development of a small, rugged system.

\section{1-2-4 Resonant Planar Waveguide Sensors}

The planar waveguide based biosensors are mostly used for optical sensing applications and are based on a shift of the propagating optical mode effective index induced by [14]:

a) The change of thickness of an adlayer of adsorbed target molecules which are transported by gaseous or liquid medium. 
b) The change of effective refractive index of the waveguide by a homogenous target medium serving as a waveguide cover.

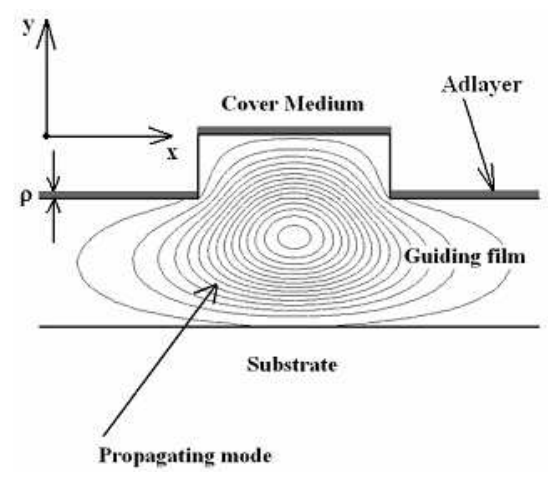

\section{Fig 4 Planar Waveguide Based Optical Sensor}

The shift of the effective refractive index produced by a change of cover medium is detected by an optical change of intensity, phase and spectrum. Mostly planar waveguide based sensors adopt single or stacked layers of waveguides to confine or couple optical power and are sensitive to the changes in evanescent field. Integrated optical sensors are fabricated using this technology due to its compactness, field ability and high sensitivity.

\section{1-3 Resonant Micro Cavity Sensors}

Resonances are peaks in intensity at certain wavelengths with respect to the input coupled wave in a localized system which is called the resonator. The peak intensities are caused by the constructive interference of wavelengths that undergo total internal reflection which is dependent on cavity dimensions. These peaks are called the resonant wavelengths of a resonator. We can interpret these modes as a wave that constructively interferes with itself after internal scattering and propagation. These modes are known as Planar Cavity Modes, Whispering Gallery Modes and Vertical Cavity Modes and shift in the spectral peaks can be measured due to analyte binding on the resonator surface. Micro cavity resonators are emerging as an attractive technology for single molecule sensors. Other applications also include switching, telecommunication and optical amplification using 
guided wave micro resonators [15].

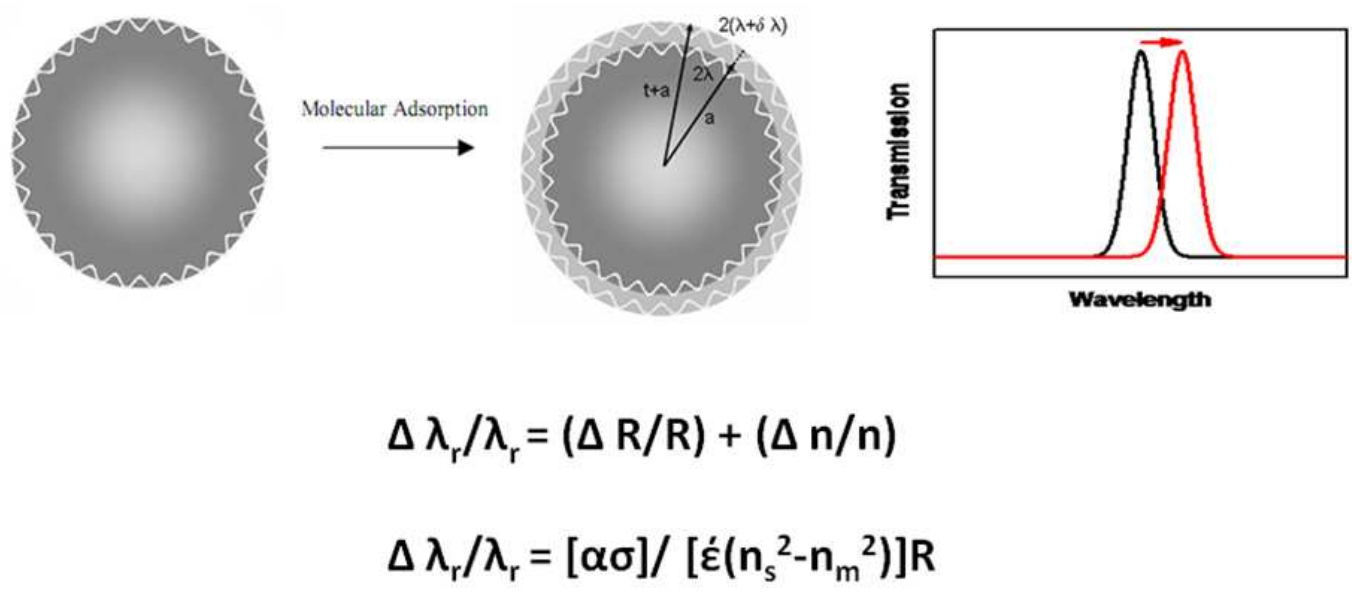

Fig 5 Resonance Peak Shift with Molecular Absorption on a Circular Micro-resonator

The main advantage is the long photon path time inside the cavity and higher interaction with target molecules which enhances the sensitivity as compared to traditional planar waveguide sensors. The coupled light into the micro resonator undergoes total internal reflection at certain wavelengths which are an integer multiple of the cavity length and produce higher intensity modes. The changes in the evanescent tail of these modes can be detected by an optical power drop at the output or a spectral shift. Peak shifts of a few picometers can be detected with single molecule attachment which makes these types of sensors among the most sensitive.

\section{1-3-1 Planar Waveguide Coupled Resonators: The SPARROW Device}

The Stacked Planar Affinity Regulated Resonant Optical Waveguide (SPARROW) biosensor is a device that exploits evanescent wave interactions and power transfer between two waveguides for bio-detection and identification [16]. The target device is a potentially inexpensive, compact, fieldable device that offers high sensitivity, environmental stability, and reduced fabrication complexity. The field of biochemical sensing technology has 
witnessed great advancements over recent years. In particular, devices that can sense and measure in a fast, efficient and cost effective way are much in demand. Our device relies on precisely tuned aluminum oxide waveguides which are fabricated on a borofloat glass substrate and separated by a thin silicon dioxide layer. Under controlled E-beam deposition techniques, thin alumina nano-layers were deposited which were in resonance with each other. The ion beam assisted deposition (IBAD) technology was used to obtain lower coupling losses and scattering losses through the densification of the films and by elimination of porous scattering centers [17]. Optical losses present in the waveguides can still interfere with the effective working of the biosensor. Hence, to overcome this issue the use of a low temperature annealing procedure was introduced which lowered the losses to a great extent.

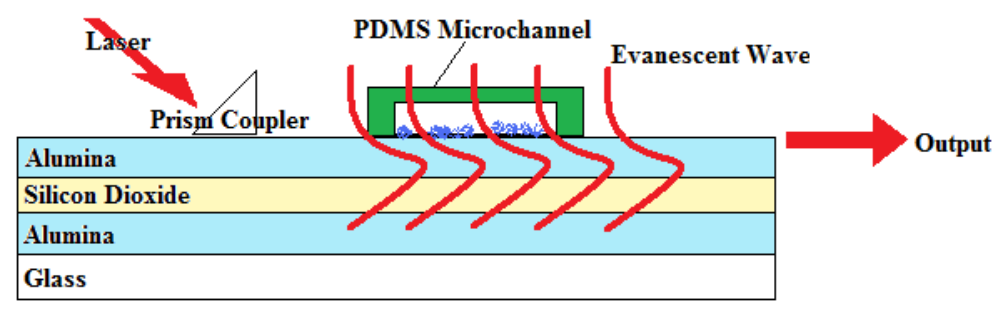

Fig 6 SPARROW Device Architecture

Light from a lasing source is launched into a thin, high index, high contrast optical waveguide stack structure, thus creating an evanescent field. The high contrast waveguide structure results in the penetration of the evanescent field tuned into the alumina layers, thus the propagation of the light is extremely sensitive to changes of the index of refraction on the surface of the waveguide. The guiding layer thickness of the waveguides used in this biosensor is on the order of $200 \mathrm{~nm}$, changing the index of refraction on the surface of the waveguide results in a relative phase difference between the TE (Transverse Electric) and TM (Transverse Magnetic) modes. This phase difference is directly dependent upon the material on the surface of the waveguide and also causes a variation in the output laser intensity. Particular, biological molecules can be detected at low concentration by attaching them to the surface of the waveguide by means of a correct antibody. 


\section{1-3-2 Whispering Gallery Mode Devices}

Optical resonances in a circular micro cavity generate propagation modes, known as whispering gallery modes (WGMs) which produce narrow spectral peaks. Micro resonators with WGMs can be used for biosensing applications as the shift in spectral peaks corresponds to the attachment of target molecules on the resonator surface. The spectral shifts are sensitive to the local refractive index changes and the experimental sensitivity is about five times greater than predicted by Mie theory. Hence, these resonators can be used as exceptional sensors for molecular detection. Micro resonator geometry varies from Microspheres to cylindrical disks or ring structures coupled to planar waveguides which can be used to pump light into the resonator. The technique is economical and greatly attracting attention for sensing and lasing applications. The WGM sensors are ideal for biosensing as they don't require labeling of molecules and show enhanced sensitivity as compared to planar waveguide based sensors. The longer photon path time in an optical cavity enhances the evanescent wave and target molecule interaction that cannot be achieved in a planar device.

\section{1-3-3 LED Resonant Devices}

Semiconductor laser diodes are favored in various applications of optical data communication, optical switching and storage devices [18, 19]. However, high power consumption has been a major obstacle in the use of these devices. Recent interest has been seen in the Resonant Cavity Light Emitting Diodes (RC-LED) which can reduce the power consumption due to high intensity resonant optical modes that are used for lasing applications. Although material properties are certainly of interest for improvement of optical characteristics, device geometry cannot be neglected. The generation of VCMs (vertical cavity modes) in circular geometry RC-LEDs is a novel area which is still under study and may open doors for sensing without any need for external optical sources. By understanding the working of the vertical cavity modes of a RC-LED, a micro-cavity design can improve the extraction efficiency by enhancing the modes and analyzing mode shifts 
for bio sensing purposes.

In a planar LED when electrical carriers are injected, photons are generated by spontaneous emission in the multi-quantum well (MQW) active region. Two mirror facets create a resonant cavity for stimulated emission to occur. Stimulated emission is initiated by an already existing photon and an emitted photon which matches the original photon not only in its wavelength but also in the direction of propagation [20]. Photons generated by spontaneous emission travel along the longitudinal direction and resonate, gaining more power as electrical input power increases. The circular cavity enhances the total internal reflection and retains the photons in the device cavity creating high intensity resonant modes which reduces the extraction efficiency. Properly designed, high Q-factors can be achieved for these optical modes resulting in an enhanced LED emission. The high internal resonance efficiency of disk cavities has received increased attention in recent years [2126].

These resonators require low threshold power and produce extremely sharp emission peaks, which are correlated with a high Q-factor (ratio of stored energy to average power loss) [27]. The disk resonators have proven to have high mode efficiency as compared to hexagonal or other polygonal resonators as there is loss of photons at the sharp edges of the hexagonal structure.

\section{1-4 Motivation and Objectives}

Optical fiber based sensors and planar waveguide based evanescent wave sensors have gained attention in the last decade $[28,29,30]$. The sensing is based on total internal reflection effect (TIR) and has advantages of fast, real time, non-destructive and sensitive detection of analyte molecules. The planar geometry is applicable to the integrated photonics technology and allows the traditional fabrication techniques.

This research focuses on the study of optical resonant structures as mesoscopic systems which provide an interaction platform between the microscopic and the 
macroscopic for sensing applications. The optical processes in these systems are leaky as they rely on interaction of molecules with their extended modes of radiation (evanescent fields). In the past decade much interest has developed in the use of optical processes in Fabry-Perot microcavities, optical fibers and dielectric spheres for sensing. The planar coupled optical structures are readily fabricated and form the basis of integrated optics but have a limited interaction of resonant modes with the environment. Looped waveguide or cylindrical waveguide geometries form a circular mode which has longer photon path length and increased interaction but pose fabrication and optical coupling challenges. These two geometries are chosen for this study, given their complementary nature an application potential. The recent advancements in Nanophotonics devices and sensors have developed a need for integrated miniature optical devices which can be used to detect at a molecular scale. The fusion of nanotechnology and photonics brings new challenges in the nanofabrication and sensing areas. The most researched technical problem in integrated photonic sensors is the sensitivity enhancement at a molecular scale producing detectable signals. The sensitivity is based on the optical losses of the resonator optical sensor and the interaction of the optical modes with the analyte molecules. Hence planar, spherical and cylindrical optical resonators are considered to address the sensitivity problem and to analyze different geometries of evanescent wave interaction with the analyte.

The first goal was to design and fabricate a aluminum oxide planar resonant stack waveguide (SPARROW) structure and to characterize the transducer performance with a limited photon-analyte sensitivity interaction platform. The work results in the first sensitivity results from such a structure for detection of aqueous sucrose solution refractive index changes and non-functional fluorescent polystyrene microsphere attachment events. The study will also result in the design, fabrication and characterization of an optically pumped GaN disk structure to study the circular WGM resonances. The cylindrical resonant cavity sensors are more compact and sensitive as compared to their planar waveguide counterparts. By enhancing the evanescent wave interaction the sensitivity and accuracy of detection is improved. 
GaN based LED's are the most extensively used for illumination because they cover a wide range of wavelengths and are primarily used in the semiconductor industry making them a favorite for integrated photonic structures. This research investigates the novel concept of generation and detection of vertical cavity modes in an electrically pumped MQW LED device with integrated Bragg reflector. LED micro resonators were simulated for characterization of cavity modes, as well as the design and placement of LED layers. Generation of VCMs in a wide band gap InGaN/GaN Multiple Quantum Well (MQW) RCLED structure that serves as both resonator and photon source are studied for enhance LED output emission and sensing applications. 


\section{Chapter 2}

\section{Theory}

\section{2-1 Planar Dielectric Waveguide}

The goal of this section is to establish the fundamental concepts and background of guided waves in a planar slab dielectric waveguide. It also explains the basic equations needed to analyze optical slab waveguide structure with a brief review of Maxwell equations, boundary conditions; TE/TM guided modes, evanescent waves and the dispersion relation.

\section{2-1-1 Maxwell's Equations and Electromagnetic Analysis}

Electromagnetic waves are generated by time-varying currents or charges and are transverse in nature. The time varying fields are electric and magnetic, which can be represented in a vector form and have both magnitude and direction. The electromagnetic waves propagation and the variation of electric and magnetic field vectors have been well established and governed mathematically using Maxwell Equations [31, 32]. The integral and differential form of these equations can be written as follows.

\section{2-1-1-1 Maxwell's Equations in Integral Form}

The integral form of Maxwell equations explains the interaction of electromagnetic phenomena with finite objects of specified shapes and boundaries in a physical environment. The integral form of Maxwell's equations is derived from its differential form by utilizing the Stock's divergence theorem [33]. 


$$
\begin{array}{ll}
\int_{l} \mathbf{E} \cdot d l=-\int_{s} \frac{\partial \mathbf{B}}{\partial t} \cdot d s & \text { (Faraday's law) } \\
\int_{l} \mathbf{H} \cdot d l=\int_{s}\left(\mathbf{J}+\frac{\partial \mathbf{D}}{\partial t}\right) \cdot d \mathbf{s} & \text { (Generalized ampere's law) } \\
\int_{s} \mathbf{D} . d \mathbf{s}=\int_{v} \rho_{\mathbf{v}} d v & \text { (Gauss's law) } \\
\int_{s} \mathbf{B} . d s=0 &
\end{array}
$$

Where:

$\mathbf{E}$ is the electric field intensity

B is the magnetic flux density

$\mathbf{H}$ is the magnetic field intensity

D is the electric flux density

$\rho_{v}$ is the volume density of free charges

$\mathbf{J}$ is the density of free current

The constitutive relations that connect $\mathbf{D}, \mathbf{B}$ and $\mathbf{J}$ with $\mathbf{E}$ and $\mathbf{H}$ for linear, isotropic and homogenous media are given by [31, 32]:

$$
\begin{array}{ll}
\mathbf{D}=\varepsilon \mathbf{E} & 1.5 \\
\mathbf{B}=\mu \mathbf{H} & 1.6 \\
\mathbf{J}=\sigma \mathbf{E} & 1.7
\end{array}
$$

Where $\varepsilon, \mu$ and $\sigma$ are scalar constants in this case, which is permittivity, 
permeability \& conductivity of any given medium respectively.

\section{2-1-1-2 Maxwell's Equations in Differential Form}

The Maxwell's equations in differential form are applied to describe the related field vectors, current densities and charge densities of electromagnetic waves at any point in space-time of a given medium. By applying Stokes divergence theorem to equation (1.1) through equation (1.4) [33]:

$$
\begin{array}{ll}
\oint_{l} \mathbf{A} \cdot d \mathbf{l}=\iint(\nabla \times \mathbf{A}) \cdot d \mathbf{s} & (\text { Stokes theorem }) \\
\oint_{s} \mathbf{A} \cdot d \mathbf{s}=\iiint(\nabla \times \mathbf{A}) \cdot d v \quad(\text { Divergence theorem })
\end{array}
$$

We get,

$$
\begin{aligned}
& \nabla \times \mathbf{E}=\frac{-\partial \mathbf{B}}{\partial t} \\
& \nabla \times \mathbf{H}=\mathbf{J}+\frac{\partial \mathbf{D}}{\partial t} \\
& \nabla . \mathbf{D}=\rho_{v} \\
& \nabla . \mathbf{B}=0
\end{aligned}
$$

The Maxwell's equations can be solved for the condition of a dielectric waveguide, having a charge free, lossless, linear and isotropic medium, where $\rho_{v}$ and $J$ will be eliminated [31, 32, 33].

$$
\nabla \times \mathbf{E}=\frac{-\partial \mathbf{B}}{\partial t}
$$




$$
\begin{aligned}
& \nabla \times \mathbf{H}=\frac{\partial \mathbf{D}}{\partial t} \\
& \nabla . \mathbf{D}=0 \\
& \nabla . \mathbf{B}=0
\end{aligned}
$$

The above Maxwell's equations represent a charge free waveguide medium and can be solved as time varying fields.

\section{2-1-1-3 Maxwell's Equations for Harmonic Fields}

A time-varying harmonic field varies sinusoidaly with time and can be represented as [34]:

$$
\begin{aligned}
& \mathbf{E}(x, y, z, t)=\operatorname{Re}\left(\mathbf{E} e^{i \omega t}\right) \\
& \mathbf{H}(x, y, z, t)=\operatorname{Re}\left(\mathbf{H} e^{i \omega t}\right) \\
& \mathbf{D}(x, y, z, t)=\operatorname{Re}\left(\mathbf{D} e^{i \omega t}\right) \\
& \mathbf{B}(x, y, z, t)=\operatorname{Re}\left(\mathbf{B} e^{i \omega t}\right)
\end{aligned}
$$

Here $\omega$ is the angular frequency of the harmonic fields, and in order to derive the Maxwell's equations for a free charge, lossless medium, we can take explicit derivatives of $\mathbf{B}$ and $\mathbf{D}$ with respect to time [34]:

$$
\begin{aligned}
& \nabla \times \mathbf{E}=i \omega \mu \mathbf{H} \\
& \nabla \times \mathbf{H}=i \omega \varepsilon \mathbf{E} \\
& \nabla . \mathbf{E}=0
\end{aligned}
$$




$$
\nabla . \mathbf{H}=0
$$

\section{2-1-1-4 Helmholtz Equation}

The wave equation can derived in its sinusoidal steady state form as Helmholtz equation. Taking curl of the Maxwell equations for harmonic fields [34]:

Taking the curl of Eq. (1.22)

$$
\begin{aligned}
& \nabla \times \nabla \times \mathbf{E}=i \omega \mu(\nabla \times \mathbf{H}) \\
& \nabla \times \nabla \times \mathbf{E}=-i \omega \mu(i \omega \varepsilon \mathbf{E}) \\
& =\omega^{2} \mu \varepsilon \mathbf{E}
\end{aligned}
$$

Using the vector identity $\nabla \times \nabla \times \mathbf{A}=\nabla(\nabla . \mathbf{A})-\nabla^{2} \mathbf{A}$, And $\nabla . \mathbf{E}=0$,

Then Equation (1.28) becomes,

$$
\nabla^{2} \mathbf{E}+\omega^{2} \mu \varepsilon \mathbf{E}=0
$$

Similarly, taking the curl of Equation (1.23)

$$
\begin{aligned}
& \nabla \times \nabla \times \mathbf{H}=i \omega \varepsilon(\nabla \times \mathbf{E}) \\
& -\nabla^{2} \mathbf{H}=\omega^{2} \varepsilon \mu \mathbf{H} \\
& \nabla^{2} \mathbf{H}+\omega^{2} \varepsilon \mu \mathbf{H}=0
\end{aligned}
$$

This is the Helmholtz equation in its steady state form. In next section the various forms of Maxwell's equations can be applied to analyze the electric and magnetic field 
vectors across any given boundary between two media.

\section{2-1-2 Boundary Conditions}

The boundary conditions are necessary in order to evaluate an electromagnetic problem which involves contiguous regions or various constitutive parameters, and the variation of field vectors $\mathbf{E}, \mathbf{D}, \mathbf{B}$ and $\mathbf{H}$ at any given interface. We will deal with the boundary conditions of a dielectric waveguide in determining the fields on either side of the boundary. The Maxwell's equations in integral form are applied [32, 34]:

$$
\begin{aligned}
& \oint \mathbf{E} . d \mathbf{l}=0 \\
& \oint \mathbf{D} . d \mathbf{s}=\text { Qenc }
\end{aligned}
$$

The boundary conditions for a rectangular and a disk structure across media 1 and 2 is analyzed. The electric field existing in a region consisting of two different dielectrics is considered as $\mathbf{E}_{\mathbf{1}}$ and $\mathbf{E}_{\mathbf{2}}$, and with respective permittivities of:

$$
\begin{aligned}
& \varepsilon_{1}=\varepsilon_{o} \varepsilon_{r 1} \\
& \varepsilon_{2}=\varepsilon_{o} \varepsilon_{r 2}
\end{aligned}
$$

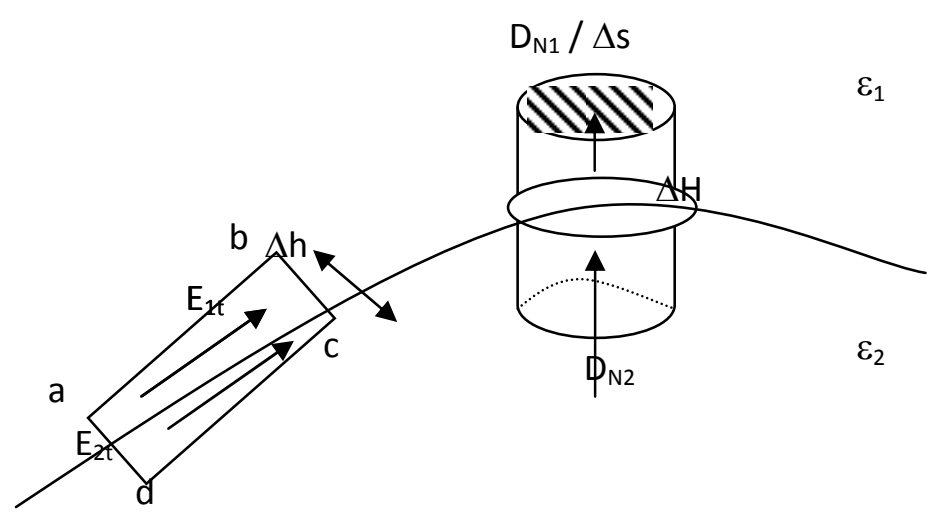

Fig 7 Dielectric-dielectric boundary across two media 
The normal component of the displacement vector $\mathbf{D}_{\mathbf{n}}$ is continuous across the boundary in the disk dielectric structure of top surface area $\Delta \mathrm{s}$ and height $\Delta \mathrm{H}$. Whereas the tangential component of electric field $\mathbf{E}_{\mathbf{t}}$ is continuous across the boundary in the rectangular dielectric structure. The Maxwell integral equation (1.33) is applied across the closed path $\mathrm{a} b \mathrm{c} \mathrm{d}$ a as shown in figure 7. Assuming that the path is small enough to ignore any variations of the electric field, we get [31-34]:

$$
\begin{aligned}
& 0=E_{1 t} \Delta l-E_{2 t} \Delta l \\
& E_{1 t}=E_{2 t}
\end{aligned}
$$

Then, the tangential component of electric field $\mathbf{E}_{\mathbf{t}}$ undergoes no change at the boundary and it is said to be continuous across the boundary.

Since,

$$
D=\varepsilon E=D_{t}+D_{n}
$$

Equations (1.35) and (1.36) can be written as:

$$
\begin{aligned}
& \frac{D_{1 t}}{\varepsilon_{1}}=E_{1 t}=E_{2 t}=\frac{D_{2 t}}{\varepsilon_{2}} \\
& \frac{D_{1 t}}{\varepsilon_{1}}=\frac{D_{2 t}}{\varepsilon_{2}}
\end{aligned}
$$

Since the tangential component of displacement vector $\mathbf{D}_{\mathbf{t}}$ undergoes some change across the interface, $\mathbf{D}_{\mathbf{t}}$ is said to be discontinuous across the interface. Similarly, by applying equation (1.34) to the disk dielectric structure of Fig 7.

As $\Delta h \rightarrow 0$, we get:

$$
\Delta Q=\rho_{s} \Delta s=D_{1 n} \Delta s-D_{2 n} \Delta s
$$




$$
D_{1 n}=D_{2 n}=\rho_{s}
$$

Here, $\rho_{\mathbf{s}}$ is the free charge density present at the boundary. If no charges exist at the interface, then $\rho_{\mathrm{s}}=0$ and therefore:

$$
\begin{aligned}
& D_{1} \cos \theta_{1}=D_{2} \cos \theta_{2} \\
& D_{1 n}=D_{2 n}
\end{aligned}
$$

Hence, the normal component of displacement vector $\mathbf{D}_{\mathbf{n}}$ undergoes no change at the boundary.

Since, $D=\varepsilon$, therefore:

$$
\varepsilon_{1} E_{1 n}=\varepsilon_{2} E_{2 n}
$$

Considering $\mathbf{D}_{\mathbf{1}}$ or $\mathbf{E}_{\mathbf{1}}$ and $\mathbf{D}_{\mathbf{2}}$ or $\mathbf{E}_{\mathbf{2}}$ making angles $\theta_{1}$ and $\theta_{2}$ with the normal to the interface as shown in figure 8 [31-34]:

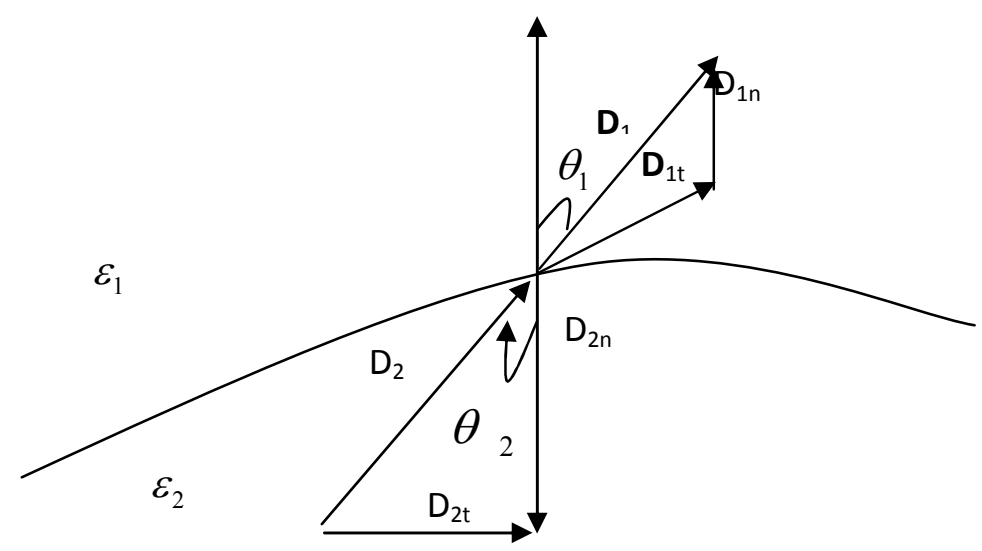

Fig 8 The Refraction of displacement vector $D$ at a dielectric-dielectric boundary

Using equations (1.40) and (1.41):

$$
\frac{\mathbf{D}_{1 t}}{\mathbf{D}_{2 t}}=\frac{\mathbf{D}_{1} \sin \theta_{1}}{\mathbf{D}_{2} \sin \theta_{2}}=\frac{\varepsilon_{1}}{\varepsilon_{2}} \text { or } \varepsilon_{2} \mathbf{D}_{1} \sin \theta_{1}=\varepsilon_{1} \mathbf{D}_{2} \sin \theta_{2}
$$




$$
\varepsilon_{2} \tan \theta_{1}=\varepsilon_{1} \tan \theta_{2} \text { or } \frac{\tan \theta_{1}}{\tan \theta_{2}}=\frac{\varepsilon_{1}}{\varepsilon_{2}}
$$

Since $\varepsilon_{1}=\varepsilon \varepsilon_{r 1}$ and $\varepsilon_{2}=\varepsilon \varepsilon_{r 2}$ :

$$
\frac{\tan \theta_{1}}{\tan \theta_{2}}=\frac{\varepsilon_{r 1}}{\varepsilon_{r 2}}
$$

This is the law of refraction of the electric field at the boundary of two medium which are free of charges [34]. This law can be explained in another form as Snell's law in the next section.

\section{2-1-3 Reflection and Refraction Analysis}

It is necessary to understand how dielectric structures exploit various optical properties of the material for the propagation of electric and magnetic fields. The laws of reflection and refraction examine the behavior of fields at a boundary between two medium.

Assuming a light wave incident on a smooth boundary between two homogeneous media of refractive indices $\mathbf{n}_{1}$ and $\mathbf{n}_{\mathbf{2}}\left(\mathrm{n}_{1}>\mathrm{n}_{2}\right)$, then a part of wave is transmitted from region $\mathbf{n}_{\mathbf{1}}$ to $\mathbf{n}_{\mathbf{2}}$. The angle of incidence is always equal to angle of reflection.

$$
\theta_{i}=\theta_{r}
$$

The angle of transmitted wave, $\theta_{\mathrm{t}}$ is related to incident angle $\theta_{\mathrm{i}}$ by Snell's law [35]:

$$
n_{1} \sin \theta_{i}=n_{2} \sin \theta_{t}
$$




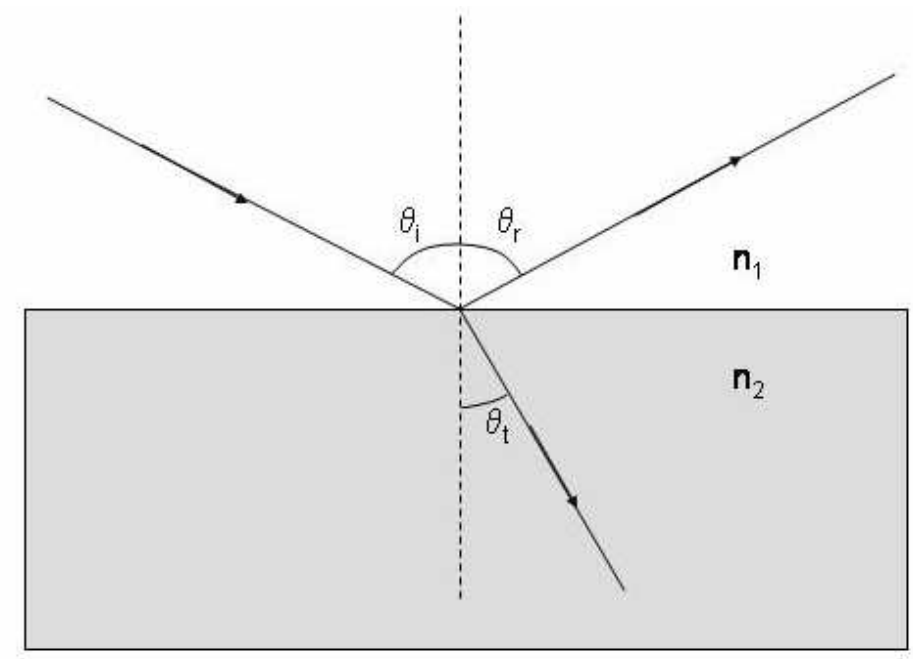

\section{Fig 9 Reflected and Refractive Wave at a Boundary Interface}

When a light beam moves from a region of higher refractive index to a region of lower refractive index then $\theta_{\mathrm{t}}>\theta_{\mathrm{i}}$ and at some particular angle of incidence, assuming that none of the beam is transmitted in the medium of lower refractive index then Snell's law is simplified to [35]:

$$
\begin{aligned}
& \sin \theta_{i}=\frac{n_{2}}{n_{1}} \\
& \theta_{c}=\sin ^{-1}\left(\frac{n_{2}}{n_{1}}\right)
\end{aligned}
$$

This relation is defined as the critical angle and for angles of incidence greater than this critical angle, the incident beam experiences 'total internal reflection' and no light is transmitted across the boundary. This is the phenomenon that is the basis of propagation of light in an optical waveguide. 


\section{2-1-4 Goos-Hanchen Effect and Evanescent Waves}

As explained in the previous section, at angles of incidence greater than the critical angle the light beam undergoes a total internal reflection and is not transmitted into the medium of lower refractive index. The Goos-Hanchen shift is the small displacement that a light beam undergoes when it is totally internally reflected at the interface of two medium It appears as if the incident light penetrates first into the lower refractive index medium and is also known as an 'evanescent wave' before being totally reflected back into the high index medium.

The resulting Goos-Hanchen displacement at the interface is given by [36]:

$$
Z_{G H}=\frac{\lambda}{\pi} \frac{\tan \theta}{\sqrt{\left(n_{1}\right)^{2}-n_{2}^{2}}}
$$

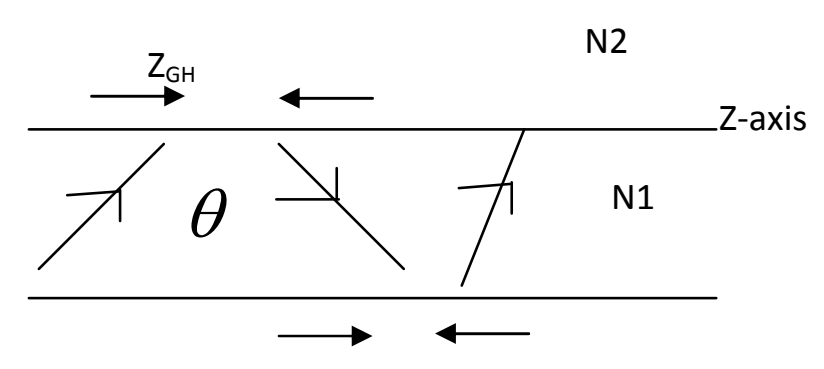

Fig 10 Goos-Hanchen Displacement at the interface of two medium

Where $\theta$ is the angle of incidence, and by differentiating equation (1.54) with respect to $n_{2}$ we get the sensitivity [36]:

$$
S_{G H}=\frac{\partial Z_{G H}}{\partial n_{2}}=\frac{k_{o}^{2}}{\pi}\left[\frac{\sec ^{2} \theta S}{\gamma_{1} \gamma_{2}}-\frac{k_{o} \tan \theta}{\gamma_{2}^{3}}\left(n_{1} S-n_{2}\right)\right]
$$

Where $S=\frac{\partial n_{1}}{\partial n_{2}}, \lambda$ is the wavelength of the guided wave and sensitivity is defined as 
the change of Goos Hanchen shift at the waveguide cladding interface through the cover medium.

The evanescent wave intensity $\boldsymbol{I}(\boldsymbol{x})$ decays exponentially with perpendicular distance from the interface [36]:

$$
\mathbf{I}(x)=\mathbf{I}_{o} e^{\frac{-x}{d}}
$$

Where, $\boldsymbol{I}_{\boldsymbol{o}}$ is the intensity at $\mathrm{x}=0$ (at the interface between two media of different refractive indices) and $\boldsymbol{d}$ is the 'Penetration Depth' that represents the effective depth of the evanescent wave:

$$
\mathbf{d}=\frac{\lambda}{4 \pi}\left(n_{1}^{2} \sin ^{2} \theta-n_{2}^{2}\right)^{-1 / 2}
$$

Here $\mathrm{d} \rightarrow \infty$ and $\theta \rightarrow \theta_{\mathrm{c}}$, the value of $\boldsymbol{I}_{\boldsymbol{o}}$ depends on $\theta$ and can be several times stronger than the original intensity of the incident beam.

\section{2-1-5 Planar Optical Waveguides}

Optical waveguides are structures that confine and guide light waves for propagation over a distance with minimal transmission losses. The phenomenon of total internal reflection causes the light rays to propagate through an optical mode in the waveguide. The optical fiber is a cylindrical waveguide which can guide multiple optical modes and is an excellent medium for propagation over longer distances. For the condition of total internal reflection the core of the fiber is made of higher refractive index as compared to the cladding. Waveguides are classified as step index and graded index, in which step index have uniform refractive index in each layer whereas graded index has a continuously varying index profile in the core. The planar waveguides confine light in one transverse dimension whereas channel waveguides confine light in both transverse directions. Discussing the behavior of electromagnetic waves in dielectric media will help 
us to analyze dielectric wave guides.

Our aim in this section is to develop the mathematical model that will enable us to analyze and design a waveguide structure. This general model can be applied to obtain the modes in a dielectric slab Waveguide.

\section{2-1-5-1 Basic Waveguide Equations}

We will design an analytical model to create a dielectric waveguide for propagation of optical energy in a given direction. We define the longitudinal axis of our waveguide as the Z- axis and design it such that energy is propagating in the waveguide in the Z-direction with a longitudinal propagation constant $\beta$.

The electric and magnetic fields in a planar waveguide can be written as:

$$
\begin{aligned}
& E(x, y, z)=E_{o}(x, y)=e^{-i \beta z} \\
& H(x, y, z)=H_{o}(x, y)=e^{-i \beta z}
\end{aligned}
$$

Using Maxwell's equations the above equations can be expanded to [31-35]:

$$
\begin{aligned}
& \left|\begin{array}{ccc}
\mathbf{i} & \mathbf{j} & \mathbf{k} \\
\frac{\partial}{\partial x} & \frac{\partial}{\partial y} & \frac{\partial}{\partial z} \\
E_{x} & E_{y} & E_{z}
\end{array}\right|=-i \omega \mu\left(H_{x} \mathbf{i}+H_{y} \mathbf{j}+H_{z} \mathbf{k}\right) \\
& \left(\frac{\partial E_{z}}{\partial y}-\frac{\partial E_{y}}{\partial z}\right) \mathbf{i}+\left(\frac{\partial E_{x}}{\partial z}-\frac{\partial E_{z}}{\partial x}\right) \mathbf{j}+\left(\frac{\partial E_{y}}{\partial x}-\frac{\partial E_{x}}{\partial y}\right) \mathbf{k} \\
& =-i \omega \mu\left(H_{x} \mathbf{i}+H_{y} \mathbf{j}+H_{z} \mathbf{k}\right) .
\end{aligned}
$$




$$
\begin{aligned}
& \left|\begin{array}{lll}
\mathbf{i} & \mathbf{j} & \mathbf{k} \\
\frac{\partial}{\partial x} & \frac{\partial}{\partial y} & \frac{\partial}{\partial z} \\
E_{x} & E_{y} & E_{z}
\end{array}\right|=i \omega \varepsilon\left(E_{x} \mathbf{i}+E_{y} \mathbf{j}+E_{z} \mathbf{k}\right) \\
& \left(\frac{\partial H_{z}}{\partial y}-\frac{\partial H_{y}}{\partial z}\right) \mathbf{i}+\left(\frac{\partial H_{x}}{\partial z}-\frac{\partial H_{z}}{\partial x}\right) \mathbf{j}+\left(\frac{\partial H_{y}}{\partial x}-\frac{\partial H_{x}}{\partial y}\right) \mathbf{k} \\
& =i \omega \varepsilon\left(E_{x} \mathbf{i}+E_{y} \mathbf{j}+E_{z} \mathbf{k}\right)
\end{aligned}
$$

Using equations (1.58) and (1.59):

$$
\begin{aligned}
& \frac{\partial E x}{\partial z}=-i \beta E x, \frac{\partial E y}{\partial z}=-i \beta E y, \frac{\partial H x}{\partial z}=-i \beta H x, \\
& \frac{\partial H y}{\partial z}=-i \beta H y,
\end{aligned}
$$

Hence we obtain the $\mathrm{x}, \mathrm{y}$ and $\mathrm{z}$ components as:

$$
\begin{array}{lcc}
\frac{\partial E z}{\partial y}=-i \beta E_{y}=-i \omega \mu H_{x} & \text { (x-component) } & 1.65 \\
-i \beta E_{x}-\frac{\partial E_{z}}{\partial x}=-i \omega \mu H_{y} & \text { (y-component) } & 1.66 \\
\frac{\partial E_{y}}{\partial x}-\frac{\partial E_{x}}{\partial y}=-i \omega \mu H \omega & \text { (z-component) } & 1.67 \\
\frac{\partial H_{z}}{\partial y}-i \beta H_{y}=-i \omega \varepsilon E_{x} & & \\
-i \beta H_{x}-\frac{\partial H_{z}}{\partial x}=i \omega \varepsilon E_{y} & \text { (x-component) } & 1.68 \\
& & \\
& \text { (y-component) } &
\end{array}
$$




$$
\frac{\partial H_{y}}{\partial x}-\frac{\partial H_{x}}{\partial y}=-i \omega \varepsilon E_{z}
$$

(z-component)

To express transverse components in terms of the longitudinal components, we can express $E_{x}, E_{y}, H_{x}, H_{y}$ in terms of $E_{z}$ and $H_{z}$ [31-35]:

$$
\begin{aligned}
& \frac{\partial H_{z}}{\partial y}+\frac{i \beta}{-i \omega \mu}\left[-i \beta E_{x}-\frac{\partial E_{z}}{\partial x}\right]=i \omega \varepsilon E_{x} . \\
& \therefore\left(i \omega \varepsilon-\frac{i \beta^{2}}{\omega \mu}\right) E_{x}=\frac{\partial H_{z}}{\partial y}+\frac{\beta}{\omega \mu} \frac{\partial E_{z}}{\partial x}
\end{aligned}
$$

Multiplying both sides of above equation by $-i \omega \mu$ :

$$
\left(\omega^{2} \mu \varepsilon-\beta^{2}\right) E_{x}=-i\left(\omega \mu \frac{\partial H_{z}}{\partial y}+\beta \frac{\partial E_{z}}{\partial x}\right)
$$

Assuming, $K^{2}=\omega^{2} \mu \varepsilon$ and $K^{2}=k^{2}-\beta^{2}$

$$
\begin{aligned}
& E_{x}=\frac{-i}{K^{2}}\left(\omega \mu \frac{\partial H_{z}}{\partial y}+\beta \frac{\partial E_{z}}{\partial x}\right) \\
& E_{y}=\frac{-i}{K^{2}}\left(\beta \frac{\partial E_{z}}{\partial y}-\omega \mu \frac{\partial H_{z}}{\partial x}\right) \\
& H_{x}=\frac{-i}{K^{2}}\left(\beta \frac{\partial H_{z}}{\partial x}-\omega \varepsilon \frac{\partial E_{z}}{\partial y}\right) \\
& H_{y}=\frac{-i}{K^{2}}\left(\beta \frac{\partial H_{z}}{\partial y}+\omega \varepsilon \frac{\partial E_{z}}{\partial x}\right)
\end{aligned}
$$


Therefore the transverse components of the electric and magnetic fields $E_{x}, E_{y}, H_{x}$, $\mathrm{H}_{\mathrm{y}}$ were expressed in terms of the longitudinal components $\mathrm{E}_{\mathrm{z}}$ and $\mathrm{H}_{\mathrm{z}}$. These equations form the basis of a planar waveguide and can be modified to define the analytic model of a slab waveguide in the next section.

\section{2-1-6 Scattering/Radiation Losses in a Planar Waveguide}

The purpose of this section is to derive an analytical expression for scattering/radiation loss in a planar dielectric waveguide. Optical transmission loss in a dielectric waveguide is dependent on absorption and scattering losses. The absorption of electromagnetic energy in a dielectric structure is described by the absorption coefficient $\alpha$ [37]:

$$
\alpha=4 \pi k / \lambda_{o}
$$

Here, $\boldsymbol{\lambda}_{\boldsymbol{b}}$ is the wavelength in free space and $\boldsymbol{k}$ is the imaginary part of the complex refractive index:

$$
n=n^{`}+i k
$$

The complex dielectric permittivity is related to the complex refractive index by:

$$
\varepsilon_{1}+i \varepsilon_{2}=(n+i k)^{2}
$$

The calculated absorption coefficient in our case is negligible, therefore losses are assumed to be totally dependent on the radiation/scattering modes.

Scattering losses are present due to in-homogeneous refractive index and due to surface roughness. The inhomogeneous refractive index and surface roughness causes the coupling of the fundamental guided mode to the radiation modes and is the main cause of power loss at the output.

The inhomogeneous refractive index causes Rayleigh scattering and can be 
described by [38]:

$$
\mathbf{S}_{L}=1.7 \times(0.85 / \lambda)^{4}
$$

The average Rayleigh scattering loss in a waveguide can be described by [39]:

$$
\partial_{s}=\iint_{A} \partial_{s}(x, y) E^{2}(x, y) d x d y
$$

Here, $\partial_{\mathrm{s}}(\mathrm{x}, \mathrm{y})$ is the Rayleigh scattering loss at point $(\mathrm{x}, \mathrm{y})$ and can be described as [39]:

$$
\begin{aligned}
& \partial_{s}(x, y)=C_{1}\left[n_{1}^{2}(x, y)-1\right] / \lambda^{4}, \text { For Core } \\
& \partial_{s}(x, y)=C_{2}\left[n_{2}^{2}(x, y)-1\right] / \lambda^{4}, \text { For Cladding }
\end{aligned}
$$

Where, $\mathrm{n}_{1}$ and $\mathrm{n}_{2}$ are refractive index of waveguide core and cladding respectively and $\mathrm{C}_{1}$ and $\mathrm{C}_{2}$ are Rayleigh scattering coefficients. The refractive index of the core is generally denoted as $\left(n_{1}+\delta n\right)$, where $\delta$ is the surface roughness which changes the core refractive index by a factor $\delta$. The scattering losses in a waveguide due to surface imperfections can be derived using perturbation theory as shown in literature by Payne and Lacey [40].

The perturbation theory is valid when $\delta<<\mathrm{d}$, where $\mathrm{d}$ is the waveguide thickness and $\delta$ is the waveguide surface roughness. The average surface roughness between any two random points separated by distance ' $u$ ' is given as $<\delta>=0$.

This describes a uniform boundary between two points with surface roughness fluctuations above and below this boundary. To understand the relative behavior of surface roughness between two random points correlation function is used. It describes the correlation between random variables at two different points in space. When measuring the same quantity of random variables between two points it's called autocorrelation function [41]. 


$$
<f(z) f(z+u)>=C(u)
$$

When the surface exhibits exponential correlation, and sample interval is one tenth of the surface correlation length [42]:

$$
<f(z) f(z+u)>=C(u)=\sigma^{2} \operatorname{Exp}\left(-|u| / l_{c}\right)
$$

For Gaussian correlation and higher sample intervals:

$$
<f(z) f(z+u)>=C(u)=\sigma^{2} \operatorname{Exp}\left(-u^{2} / l_{c}^{2}\right)
$$

Here $1_{c}$ is the correlation length over which the function $C(u)$ falls to $1 / e$ of its original value and $\sigma^{2}$ is the standard deviation of surface roughness equal to $\left\langle\delta^{2}>\right.$. The surface roughness and surface correlation can be related using the Fourier transform to get the power spectrum $\mathrm{S}(\gamma)$ which is related to the scattering loss [41, 42].

$$
\begin{aligned}
& C(u)=\int_{-\infty}^{\infty} e^{\gamma i u} S(\gamma) d \gamma \\
& S(\gamma)=\frac{1}{2} \pi \int_{-\infty}^{\infty} e^{\gamma u} C(u) d u
\end{aligned}
$$

Hence the power spectrum can be derived in the form of a Lorentzian when the surface is exponential:

$$
S(\gamma)=\left(\delta^{2} / \pi\right)\left(l_{c} / l+l_{c}^{2} \gamma^{2}\right)
$$

As $1_{c} \rightarrow 0$, we have a constant power spectrum. When the surface is Gaussian then:

$$
S(\gamma)=\left(\delta^{2} l_{c} / 2 \sqrt{\pi}\right) \exp \left(-l_{c}^{2} \gamma^{2} / 4\right)
$$

For surface roughness without any correlation, all spatial frequencies are radiated independently: 


$$
P_{r a d}=\int S(\gamma) d \gamma
$$

The frequencies that lead to significant loss are $\beta-\mathrm{kn}_{2}<\gamma<\beta+\mathrm{kn}_{2}$ [43]:

$$
\mathrm{P}_{\mathrm{rad}}=\beta-\mathrm{kn}_{2} \int^{\beta+\mathrm{kn} 2} \mathrm{~S}(\gamma) \mathrm{d} \gamma
$$

The magnitude of the scattering radiation is scattered at an angle $\theta$ from the waveguide axis:

$$
\gamma=\beta-\mathrm{kn}_{2} \cos \theta
$$

Summing over all radiation angles:

$$
\mathrm{P}_{\mathrm{rad}}=0 \int^{\pi} \mathrm{S}\left(\beta-\mathrm{kn}_{2} \cos \theta\right) \mathrm{d} \theta
$$

Using Beer Lamberts law the attenuation factor for power loss in a waveguide can be expressed as:

$$
\mathrm{P}=\mathrm{P}_{\mathrm{o}} \mathrm{e}^{-\alpha \mathrm{L}}
$$

Here $\mathrm{P}_{\mathrm{o}}$ is the initial power and $\mathrm{L}$ is the waveguide length. It can also be written as:

$$
\alpha=1 / \mathrm{L}\left(\mathrm{P}_{\mathrm{o}}-\mathrm{P} / \mathrm{P}_{\mathrm{o}}\right)
$$

The attenuation factor can be written as scattered power in the substrate and cladding [44]:

$$
\begin{aligned}
& \alpha=1 / \mathrm{L} 0 \int^{\pi}\left(\mathrm{P}_{\text {sub-rad }} \alpha_{1}\right) \mathrm{d} \theta+1 / \mathrm{L} 0 \int^{\pi}\left(\mathrm{P}_{\text {clad-rad }} \alpha_{2}\right) \mathrm{d} \theta \\
& \alpha=\left(\delta_{\mathrm{c}}{ }^{2} 1_{\mathrm{c}} / \lambda^{3}\right)\left(\mathrm{n}_{1}{ }^{2}-\mathrm{n}_{2}{ }^{2}\right) \mathrm{E}_{\mathrm{c}}{ }^{2} 0 \int^{\pi}\left(\mathrm{C} /\left[1+\left(\beta-\mathrm{n}_{2} \times \mathrm{k} \times \cos \theta\right)^{2} 1_{\mathrm{c}}{ }^{2} \mathrm{~d} \theta+\right.\right. \\
& \left(\delta_{\mathrm{s}}{ }^{2} 1_{\mathrm{s}} / \lambda^{3}\right)\left(\mathrm{n}_{1}{ }^{2}-\mathrm{n}_{0}{ }^{2}\right) \mathrm{E}_{\mathrm{s}}{ }^{2} 0 \int^{\pi}\left(\mathrm{S} /\left[1+\left(\beta-\mathrm{n}_{0} \times \mathrm{k} \times \cos \theta\right)^{2} 1_{\mathrm{s}}{ }^{2} \mathrm{~d} \theta\right.\right.
\end{aligned}
$$

Here $1_{c}$ and $1_{s}$ are correlation lengths, $E_{c}$ and $E_{s}$ are electric fields at cladding and substrate. $\beta$ is the propagation constant and $\mathrm{C}$ and $\mathrm{S}$ are coefficients related to waveguide indices, angle, wavelength and thickness. The integrated angle from 0 to $\pi$ sums the radiated 
power in all directions. For a single TE mode in a symmetric waveguide $\left(\mathrm{n}_{0}=\mathrm{n}_{2}\right)$ the attenuation factor can be written as [45]:

$$
\begin{aligned}
& \alpha=\left(1 / \rho^{5} \beta\right)\left(\mathrm{V}^{2} \mathrm{U}^{2} \mathrm{~W} / 1+\mathrm{W}\right) 0 \int^{\pi}\left(\delta_{\mathrm{c}}^{2} 1_{\mathrm{c}} / \pi\left[1+\left(\beta-\mathrm{n}_{2} \times \mathrm{k} \times\right.\right.\right. \\
& \cos \theta)^{2} 1_{\mathrm{c}}^{2} \mathrm{~d} \theta
\end{aligned}
$$

Here $\mathrm{V}=\mathrm{k} \rho\left(\mathrm{n}_{1}{ }^{2}-\mathrm{n}_{2}{ }^{2}\right)^{1 / 2}=\sqrt{ } \mathrm{U}^{2}+\mathrm{W}^{2}$ is the degree of guidance, $\rho$ is the half width of the waveguide, $U=\rho\left(k^{2} n_{1}^{2}-\beta^{2}\right)^{1 / 2}$ and $W=\rho\left(\beta^{2}-k^{2} n_{2}^{2}\right)^{1 / 2}$. This result can be applied to more general asymmetric waveguide and having two components of the attenuation factor for substrate $\left(\mathrm{n}_{0}\right)$ and cladding $\left(\mathrm{n}_{2}\right)$ when $\left(\mathrm{n}_{0} \neq \mathrm{n}_{2}\right)$.

$$
\begin{aligned}
& \alpha_{\mathrm{wg}}=\left(1 / 2 \rho_{1}^{5} \beta\right)\left(\mathrm{V}_{1}^{2} \mathrm{U}_{1}^{2} \mathrm{~W}_{1} / 1+\mathrm{W}_{1}\right) 0 \int^{\pi}\left(\delta_{\mathrm{c}}{ }^{2} 1_{\mathrm{c}} / \pi[1+(\beta-\right. \\
& \left.\mathrm{n}_{0} \times \mathrm{k} \times \cos \theta\right)^{2} 1_{\mathrm{c}}^{2} \mathrm{~d} \theta+\left(1 / 2 \rho_{2}^{5} \beta\right)\left(\mathrm{V}_{2}^{2} \mathrm{U}_{2}^{2} \mathrm{~W}_{2} / 1+\mathrm{W}_{2}\right) \\
& 0 \int^{\pi}\left(\delta_{\mathrm{c}}{ }^{2} 1_{\mathrm{d}} / \pi\left[1+\left(\beta-\mathrm{n}_{2} \times \mathrm{k} \times \cos \theta\right)^{2} 1_{\mathrm{c}}{ }^{2} \mathrm{~d} \theta\right.\right.
\end{aligned}
$$

Hence $\alpha$ denotes the total scattering loss due to surface roughness causing radiation modes in the substrate and cladding. The theoretical plot for the scattering loss can be simulated and plotted as a function of surface roughness.

\section{2-2 Dielectric Slab Optical Waveguides}

The dielectric slab waveguides are the simplest and mostly used waveguide configuration. Two types of optical modes present are the guided mode and radiation mode. This waveguide structure is important to understand the light guiding properties and lays a basis for more complicated structures [46, 47, 48, 49].

\section{2-2-1 Three Layer Step Index Dielectric Slab Waveguide}

A three layer step index planar dielectric waveguide is shown in the figure below. The three regions shown are core, cladding or superstrate and the substrate. The core has 
higher refractive index and light wave propagates here in an optical mode.

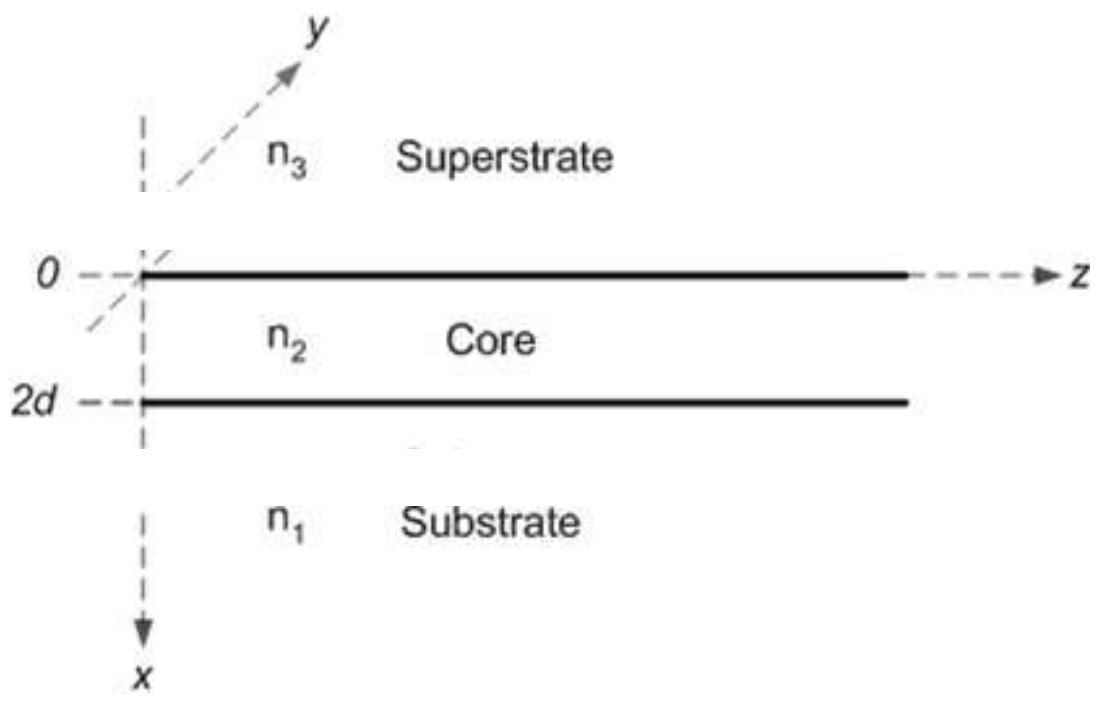

\section{Fig 11 A Three-Layer Dielectric Slab Waveguide}

We can analyze the dielectric slab waveguide and obtain expressions for the optical guided modes. The mode propagation is in the z-direction as shown in Figure 11. The slab is assumed to be infinitely extended in the y axis and by symmetry there is no variation in the field distribution in y direction. Hence the planar waveguide equations can be simplified to [46-49]:

$$
\begin{aligned}
& E_{x}=\frac{i \beta}{K^{2}}\left(\frac{\partial E_{z}}{\partial x}\right) \\
& E_{y}=\frac{i \omega \mu}{K^{2}}\left(\frac{\partial H_{z}}{\partial x}\right) \\
& H_{x}=\frac{-i \beta}{K^{2}}\left(\frac{\partial H_{z}}{\partial x}\right) \\
& H_{y}=\frac{-i \omega \mu}{K^{2}}\left(\frac{\partial E_{z}}{\partial x}\right)
\end{aligned}
$$

These equations form the electric and magnetic fields of an optical waveguide. 


\section{2-2-2 Guided Modes of the Symmetric Waveguide}

A symmetric slab waveguide has a substrate and superstrate of the same refractive index and higher index core. There are a finite number of guided modes supported by the waveguide and infinite number of radiation modes. The higher index of the core satisfies the condition of Total Internal Reflection (TIR). The modes can be odd or even in their transverse field of distribution as shown in the figure. The numbers of guided modes supported are dependent on the thickness $2 \mathrm{~d}$, the wavelength $\lambda$ and indices of refraction of the three layers.

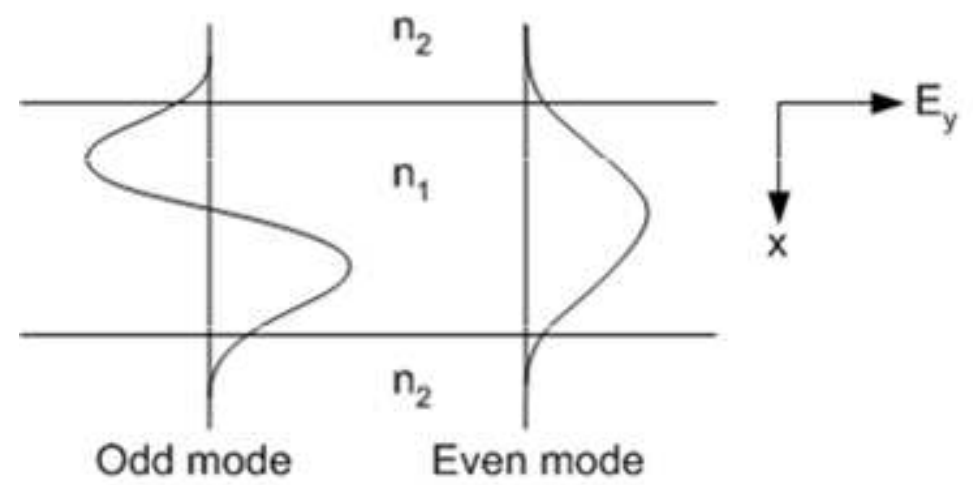

Fig 12 Electric Field Distribution of different modes in a Symmetric Slab Waveguide

\section{2-2-2-1 Transverse Electric (TE) Guided Modes}

In order to obtain the modal solution of the wave propagation in the $\mathrm{z}$ direction of the waveguide, the wave equation can be solved to obtain the Helmholtz equation:

$$
\frac{\partial^{2} E_{y}}{\partial x^{2}}+\left(\omega^{2} \mu \varepsilon-\beta^{2}\right) E_{y}=0
$$

Using the above equation and imposing the boundary conditions at the substratecore and superstrate-core interfaces, the modal solutions and propagation constants can be 
obtained. Hence the single non-zero electric field component $E y$ of the TE polarized wave can be obtained. Using Maxwell's equations, the corresponding two non-zero components of the magnetic field $H x$ and $H y$ can also be obtained. The field components of the TE polarized waves are given by [41]:

$$
\begin{aligned}
& E_{y}=A e^{-q x}, x \geq 0 \\
& E_{y}=A \cos (q x)+B \sin (q x), 0 \geq x \geq-2 a \\
& E_{y}=(A \cos (2 a q)-B \sin (2 a q)) e^{p(x+2 a)}, x \geq-2 a \\
& H_{x}=\frac{-\beta}{\omega \mu_{O}} E_{y} \\
& H_{z}=\frac{i \quad \partial E_{y}}{\omega \mu_{O} \partial x}
\end{aligned}
$$

Applying the boundary conditions for the continuity of $\mathrm{Hz}$, the following $\mathrm{TE}$ eigenvalue equation can be obtained [46-49]:

$$
\tan (2 a q)=\left(q^{2}+p q\right) /\left(q^{2}-p q\right)
$$

Where $\mathrm{q}^{2}=\mathrm{k}_{\mathrm{o}}{ }^{2} \mathrm{n}_{1}{ }^{2}-\beta^{2}$ and, $\mathrm{p}^{2}=\beta^{2}-\mathrm{k}_{\mathrm{o}}{ }^{2} \mathrm{n}_{2}{ }^{2}$

This equation relates wavelength, refractive indices, and core thickness and propagation constants. An example of the TE mode patterns for a three-layer symmetric slab waveguide is shown in the figure below. 


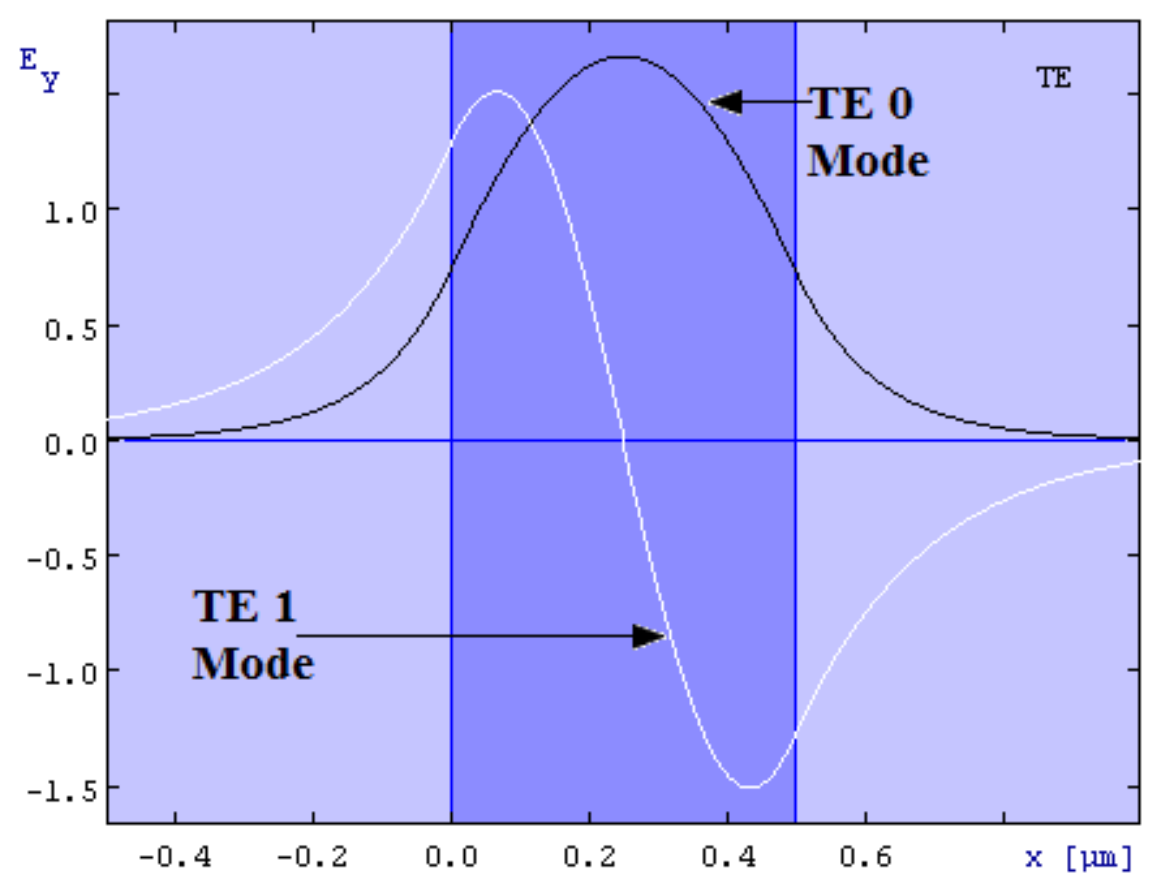

Fig 13 TE Mode Patterns of a Symmetric Waveguide

\section{2-2-2-2 Transverse Magnetic (TM) Guided Modes}

Similarly for the case of Transverse Magnetic modes the non vanishing field components are $\mathrm{H}_{\mathrm{y}}, \mathrm{E}_{\mathrm{x}}$ and $\mathrm{E}_{\mathrm{z}}$. The Helmholtz equation given becomes [41]:

$$
\frac{\partial_{2} H_{y}}{\partial z^{2}}+\left(\omega^{2} \mu E-\beta^{2}\right) H_{y}=0
$$

$\mathrm{E}_{\mathrm{x}}$ and $\mathrm{E}_{\mathrm{z}}$ in terms of $\mathrm{H}_{\mathrm{y}}$ are given by:

$$
\begin{aligned}
& E_{x}=\frac{\beta}{\omega \varepsilon} H_{y} \\
& E_{z}=\frac{-i}{\omega \varepsilon} \frac{d H_{y}}{d x}
\end{aligned}
$$

These represent the electric and magnetic fields for transverse magnetic modes of a planar slab waveguide. The waveguides coupling for a more complicated case of a multiple 
slab waveguide can be seen in the next section.

\section{2-2-3 Coupled Mode Theory}

Optical power can be transferred between the resonant waveguides. If fields of the two waveguides overlap then light can be coupled from one into the other. Coupled mode theory has been applied to analyze the electromagnetic wave propagation in integrated optics and the propagation parameters of an electromagnetic wave in the waveguide change at perturbations. The analysis of coupled waveguide system is based on the modes, propagation constants and amplitudes of the wave in individual waveguides. If the mode profiles are independent of each other (orthogonal) then orthogonal coupled theory applies, otherwise it is non-orthogonal and the solution is obtained for both the cases from the linear superposition of the waveguide modes. If the device consists of weakly coupled waveguides, then similar propagation constants are used and orthogonal coupled mode theory can be applied. Consider two guided modes which have the amplitude $\mathrm{a}_{1}$ and $\mathrm{a}_{2}$ respectively with implicit time dependence $\exp (j w t)$.

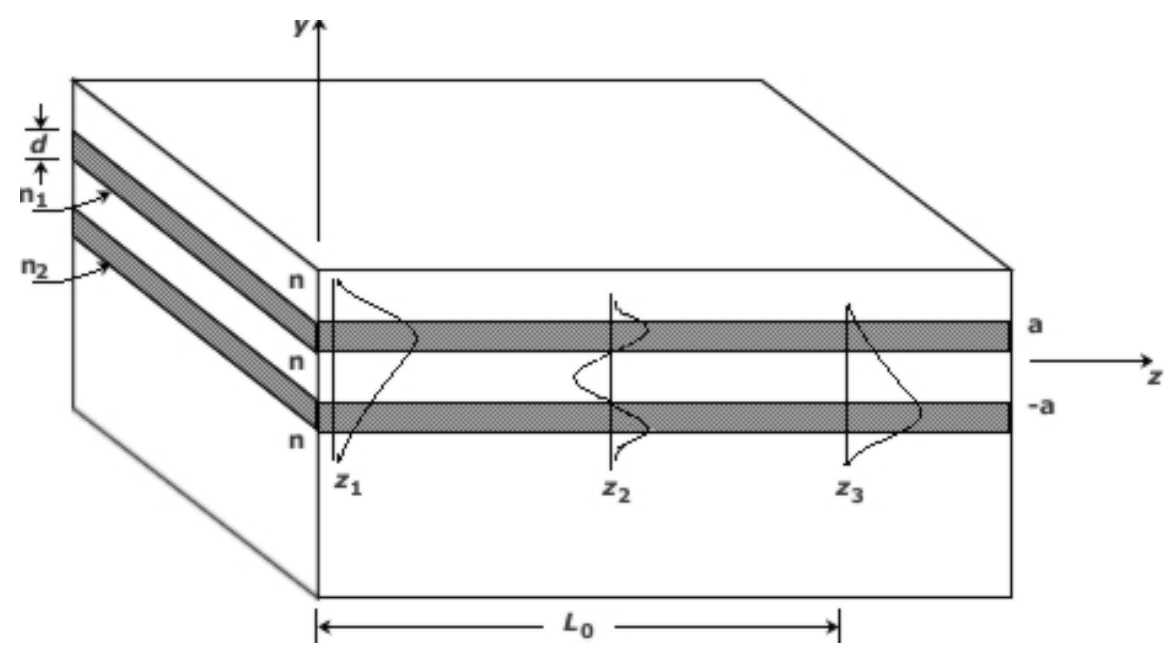

Fig 14 Coupled Waveguides and propagation of modes [50]

If they are infinitely apart from each other then they would obey the equations [41]: 


$$
\begin{aligned}
& \frac{d a_{1}}{d z}=-j \beta_{1} a_{1} \\
& \frac{d a_{2}}{d z}=-j \beta_{2} a_{2}
\end{aligned}
$$

Where $\beta 1$ and $\beta 2$ are the propagation constants, and if the waveguides are brought close, the evanescent fields would interact with each other and hence mode coupling occurs. If the coupling is weak then equation would become [41]:

$$
\begin{aligned}
& \frac{d a_{1}}{d z}=-j\left(\beta_{1}+K_{11}\right) a_{1}-j K_{12} a_{2} \\
& \frac{d a_{2}}{d z}=-j\left(\beta_{2}+K_{22}\right) a_{2}-j K_{21} a_{1}
\end{aligned}
$$

Where, $\left(\mathrm{K}_{12}, \mathrm{~K}_{21}\right)$ and $\left(\mathrm{K}_{11}, \mathrm{~K}_{22}\right)$ are the mutual and self coupling coefficients respectively. If the waveguides are lossless then the total power has to be determined constant. Hence,

$$
\begin{aligned}
& \mathbf{P}(z)=a_{1}^{2}+a_{2}^{2} \\
& \frac{d \mathbf{P}(z)}{d z}=0 \\
& K_{12}=K_{21}=K
\end{aligned}
$$

For the uniform couplers both the propagation constants and the coupling coefficients are independent of $\mathrm{z}$ and hence equations above can be solved analytically. For simplification we assume [41]:

$$
a_{1}(z)=a_{1} \exp \left\{-j\left(\beta_{1}+K_{11}+\beta_{2}+K_{22}\right) z / 2\right\}
$$




$$
\begin{aligned}
& \frac{d a_{1}}{d z}=-j\left(\delta a_{1}-K a_{2}\right) \\
& \frac{d a_{2}}{d z}=-j\left(\delta a_{2}-K a_{1}\right) \\
& \delta=\left(\beta_{1}+K_{11}-\beta_{2}-K_{22}\right) / 2
\end{aligned}
$$

Thus the beta values can be written as [41]:

$$
\begin{aligned}
& \beta_{\mathrm{s}}=\beta_{o}+\mathrm{S} \\
& \beta_{\mathrm{a}}=\beta_{o}-\mathrm{S} \\
& S=\sqrt{\left(\delta^{2}+k^{2}\right)} \\
& \beta=\left(\beta_{1}+K_{11}+\beta_{2}+K_{22}\right) / 2
\end{aligned}
$$

Assuming only one waveguide is excited then $\mathrm{a}_{1}=1$ and $\mathrm{a}_{2}=2$, the power in waveguides can be written as [41]:

$$
\begin{aligned}
& P_{1}(z)=\cos ^{2}(S z)+\cos ^{2}(\eta) \sin ^{2}(S z) \\
& P_{2}(z)=\sin ^{2}(\eta) \sin ^{2}(S z)
\end{aligned}
$$

Where, $\eta=\tan ^{-1}(\mathrm{k} / \delta)$

At $\mathrm{z}=$ Lc maximum power transfers from waveguide 1 to waveguide 2 and this Lc is known as coupling length and is given by $\mathrm{L}_{\mathrm{c}}=\pi / 2 \mathrm{~S}$.

Coupling length is the distance at which maximum power transfer occurs from one waveguide to the other waveguide. If the coupling length is higher the power transfer rate is slow and with lower coupling length the device would be prone to noise. Depending on the 
coupling length a suitable interaction length for sensing would be selected. A higher interaction length and lower coupling lengths would result in lot of power transfer from one waveguide to other and may result in higher losses.

\section{2-2-4 Sensitivity Evaluation for TE modes}

A slab waveguide structure can be constructed as an evanescent field sensor for which the waveguide modes act as the sensing feature. The guided electromagnetic field of the waveguide mode extends as an evanescent field into the cladding and substrate media and senses any changes of effective refractive index of the waveguide surface.

The effective refractive index of the propagating mode depends on the structure parameters, e.g. the guiding layer thickness and dielectric permittivity and magnetic permeability of the media constituting the waveguide. As a result, any change in the refractive index of the covering medium results in a change in the effective refractive index of the guiding mode. The basic sensing principle of the planar waveguide sensor is to measure the changes in the effective refractive index due to changes in the refractive index of the covering medium [51].

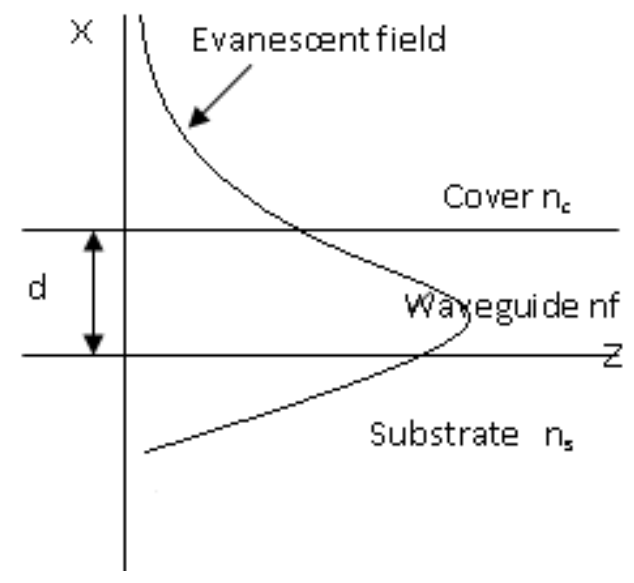

Fig 15 Schematic representation of slab waveguide sensor

Assuming a homogeneous change of index in the cover medium, the sensitivity is 
defined as the change in the effective refractive index through the cover medium [52]:

$$
S=\frac{\partial N}{\partial n_{c}}
$$

In our waveguide structure, with a constant $\boldsymbol{k}_{\boldsymbol{o}}$ (wave number) and $\boldsymbol{n}_{\boldsymbol{s}}$ (substrate index), and varying $\boldsymbol{n}_{c}$ (cover index), $\boldsymbol{N}$ (Effective index of waveguide stack), and $\gamma_{c}, \gamma_{f}, \gamma_{s}$ (Propagation constants), we find $S^{-1}=\frac{\partial n_{c}}{\partial N}$ by differentiating the dispersion relation, we get [52]:

$$
S_{T E}=\frac{\partial N}{\partial n_{c}}=\frac{n_{c} \gamma_{f}^{2} \gamma_{s} \gamma_{c}}{N k_{0}\left(\gamma_{f}+\gamma_{c}\right)\left(d \gamma_{s} \gamma_{c}+\gamma_{c}+\gamma_{s}\right)}
$$

For a homogeneous analyte surface loading in the cover medium, the cover index and effective index of the stack are proportional to the change of propagation constants and to the TE modes of the waveguide. Hence, sensitivity of the TE mode of a slab waveguide sensor can be evaluated using the above relation.

\section{2-2-5 Resonant Waveguide Structure as Biosensors}

The optical modes of the waveguide resonators extend outside the cavity as evanescent waves and changes in the refractive index on the surface can produce a shift of the resonance wavelengths which can be used for biosensing purposes. The light propagation in optical modes undergoes total internal reflection (TIR) based on Snell's law and reflection angle above the critical angle [35]:

$$
\begin{aligned}
& n_{1} \sin \theta_{1}=n_{2} \sin \theta_{2} \\
& \theta_{\text {critical }}=\sin ^{-1}\left(n_{2} / n_{1}\right)
\end{aligned}
$$

For total internal reflection to take place it is required that $\mathrm{n}_{1}>\mathrm{n}_{2}$, where $\mathrm{n}_{1}$ is the 
material's (core) refractive index and $\mathrm{n}_{2}$ is the surrounding material refractive index (cladding). The total internal reflection creates an evanescent field in the surrounding medium and decays exponentially. The interaction of this evanescent wave with refractive index changes can be detected and used for sensing purposes. The evanescent field is described as [35]:

$$
\begin{aligned}
& E(x)=E_{o} \exp \left(-x / d_{p}\right) \\
& d_{p}=\lambda /\left(2 \pi \sqrt{n_{1}^{2} \sin ^{2} \theta-n_{2}^{2}}\right)
\end{aligned}
$$

Here $\boldsymbol{d}_{\boldsymbol{p}}$ is the penetration depth where the evanescent field intensity drops to $1 / \mathrm{e}$ of its maximum value from the interface $(\mathrm{x}=0)$. $\boldsymbol{E}_{\boldsymbol{o}}$ is the field intensity at the interface, $\boldsymbol{\lambda}$ is the wavelength of light and $\theta$ is the angle of incidence at the interface. The optical output of the waveguide modes are proportional to index changes in the evanescent field and can be detected. Also spectral changes in the optical output can be detected to get a signature analysis of the bound molecules.

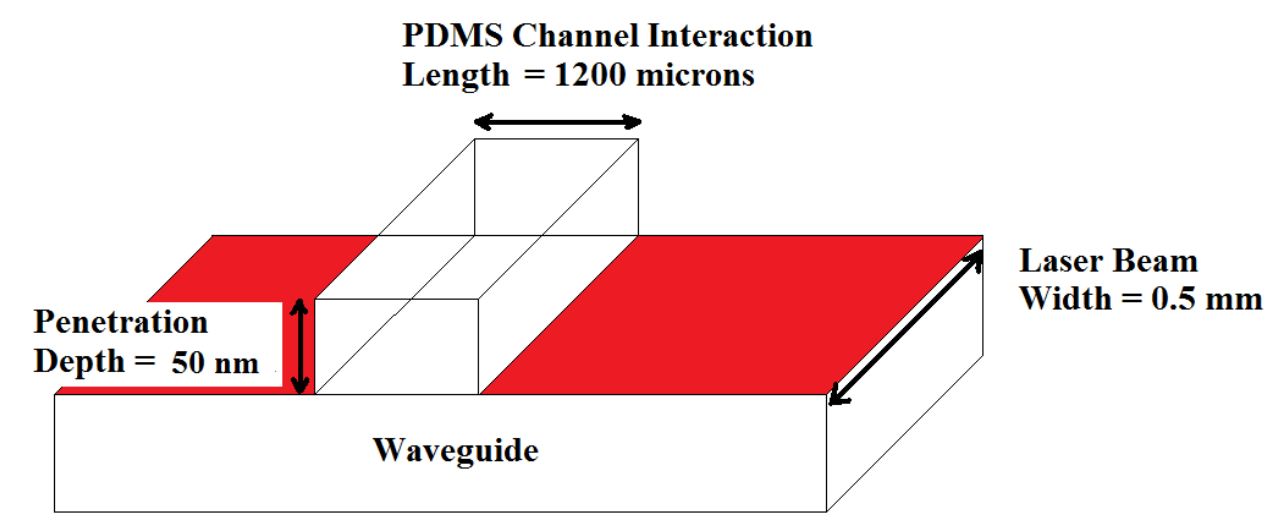

Fig 16 Evanescent wave $(50 \mathrm{~nm})$ interaction with analyte in microchannel $(1200 \mu \mathrm{m})$

The figure above shows the interaction volume of the evanescent field with the analyte in microchannel. At various concentrations of analyte aqueous solutions the amount of surface loading can be calculated. For a laser beam width of $0.5 \mathrm{~mm}$, channel length of $1200 \mu \mathrm{m}$ and evanescent field of $50 \mathrm{~nm}$ the total amount of sucrose (for a $0.1 \%$ solution) 
present in the interaction area is $50 \mathrm{pg} / \mathrm{mm}^{2}$. The optical transducer changes will be calculated to the steps of $50 \mathrm{pg} / \mathrm{mm}^{2}$ later shown in the results section and a minimal change of optical power to analyte changes will be measured as the limit of detection (LOD) of the transducer.

From a measurement of the optical output the properties and amount of this analyte can be determined. Two types of sensing mechanisms can be used. In homogenous analyte sensing a uniform layer of solution is covered on the resonator device surface and the output intensity and wavelength shift corresponds to all materials present in the solution. Whereas a molecular binding on the resonator surface can only detect the presence of that molecule and corresponding optical output intensity can be detected, which makes this technology as one of the most sensitive label free molecular analysis.

\section{2-3 Optical Modes of Dielectric Spheres}

\section{2-3-1 Microsphere Resonators}

The propagation of light in a microsphere is similar to that in a planar dielectric waveguide. The microsphere resonator is like a circular waveguide with the propagation of modes along the circumference. Similar to planar waveguides light is confined at the high index region and decays exponentially in the surroundings which form the evanescent field region. When the phase shift after one circular trip is an integer multiple of $2 \pi$, the light will undergo constructive interference and gain intensity and will become a resonant mode of the spherical resonator. These resonant modes are knows as Whispering gallery modes [53]. The resonance condition is described by:

$$
\lambda_{\text {resonance }}=\left(2 \pi R n_{\text {eff }}\right) / \mathrm{m}
$$




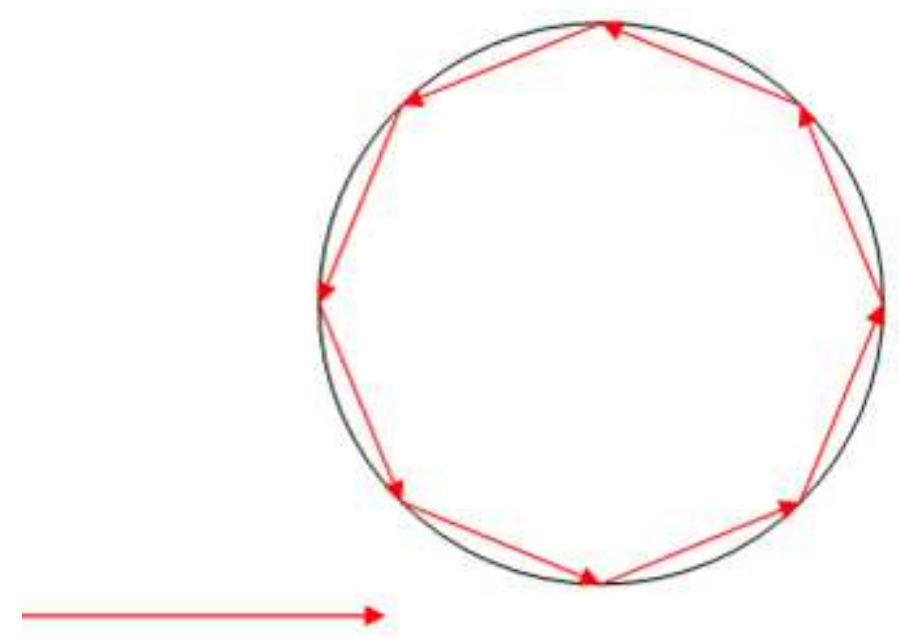

Fig 17 Whispering gallery mode in a microsphere

Where $\mathrm{R}$ is the radius of the sphere, $\mathrm{n}_{\mathrm{eff}}$ is the effective refractive index of the material and $\mathrm{m}$ is the integer number of wavelengths which can fit inside the circular cavity. Generally microcavities are coupled to an excitation waveguide which couples light into the cavity through the evanescent tail of the waveguide. The transmission spectrum of the waveguide will show dips at the resonant frequencies of the microcavity. The spectral distance between two modes with a subsequent mode number $\mathrm{m}$ is called the free spectral range (FSR) and is given by the equation [53]:

$$
\operatorname{FSR}=\lambda^{2} /\left(2 \pi \mathrm{R} \mathrm{n}_{\mathrm{eff}}\right)
$$

The width of the resonance peaks is described by their quality factor Q, defined as the ratio of the energy stored in the resonator to the energy loss per cycle. The $\mathrm{Q}$ factor can be calculated with the formula [53]:

$$
\mathrm{Q}=\lambda_{\text {resonance }} / \delta \mathrm{FWHM}
$$

Where $\delta$ FWHM is the width of the resonance peak/dip at half of its maximum value, hence a high $\mathrm{Q}$ factor shows a narrow spectral peak. High $\mathrm{Q}$ factors are necessary for a resonator to be used as a sensor and can increase the sensitivity to detect the minimal wavelength shifts. The most important factor to improve the $\mathrm{Q}$ factor is to reduce the 
resonant mode losses which occur at the rough surfaces and cause power to scatter out of the cavity. The surface roughness can be minimized by better fabrication techniques. Bending losses will increase with smaller radii and hence higher radius rings can be used. The microspheres exhibit a shift in wavelength when the refractive index of the surrounding material or the material inside the cavity changes and may be utilized as biosensors [54]. Generally the Q factors of 1500 and higher are required to make a useful biosensor as the detection limit of most spectrometers is around $0.3 \mathrm{~nm}$. Anything lower than $1500 \mathrm{Q}$ will be out of the reach of the detection limit and will be reported as noise.

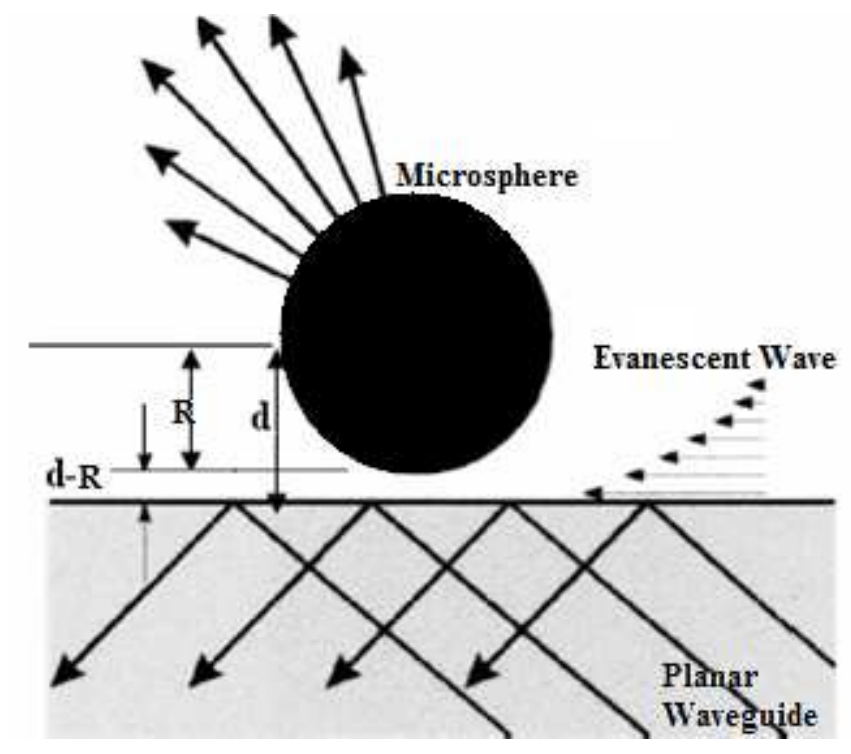

Fig 18 Evanescent coupling from a planar waveguide to microsphere modes

Besides the internally driven whispering gallery modes, the microspheres also encounter scattering/radiation modes similar to planar waveguides. This scattered light intensity couples out of the spheres by evanescent wave tail and the surface roughness. The scattered intensity from the microsphere is also sensitive to the size and refractive index of the microspheres as well as the effective refractive index of the medium surrounding the microspheres. The polystyrene microspheres used in this work are chosen due to their relatively high index of refraction compared to other common microsphere materials (i.e. silica). As shown in figure 18 the microsphere is located at distance (d-R) from the surface of a planar waveguide, $\mathrm{d}$ is the distance between the center of the microsphere and interface, 
$\mathrm{R}$ is the microsphere radius. This change in height is measured as a change in the scattered intensity from the microsphere. The losses in microspheres due to various optical modes are seen in the next section in detail.

\section{2-3-2 Loss Mechanisms (Intrinsic and Extrinsic)}

For an evanescently coupled planar waveguide-microsphere system the total scattering losses in a microsphere will be the sum of losses due to:

1) Microsphere intrinsic scattering and absorption losses

2) Microsphere surface radiation losses

In this section we will focus on the microsphere internal and surface scattering losses. As the evanescent wave of the planar waveguide couples in to the Whispering Gallery Modes of the microsphere resonator, the scattering losses are not just limited to the evanescent field region but throughout the microsphere. The losses are present due to internal scattering or absorption and external surface radiation $[55,56,57,58]$. We will derive the analytic expressions assuming that there are minimum or no coupling losses in the microsphere-waveguide system.

\section{2-3-2-1 Microsphere Intrinsic Scattering and Absorption Losses}

The intrinsic losses in the microsphere resonators have been reported previously to be dependent on the whispering gallery modes and on the $\mathrm{Q}$ factors of the microcavity.

$$
\mathrm{Q}=2 \pi \mathrm{n} / \alpha \lambda
$$

Here, $\mathrm{n}$ is the refractive index, $\alpha$ is the attenuation coefficient and $\lambda$ is the wavelength. This approach is purely based on the microsphere bulk losses and didn't include the scattering losses due to WGM or surface radiation modes, which we will discuss 
in the next section. To approach this problem the sphere volume is divided into a small element $\boldsymbol{d}_{\boldsymbol{v}}$. The fluctuations in the dielectric constant are caused due to inhomogeneities of the index [55-58]:

$$
\varepsilon(\mathrm{r})=\delta \varepsilon(\mathrm{r})+\varepsilon^{0}
$$

And cause the field mode to behave as a dipole and hence re-radiate light in all directions. This can be derived using the Rayleigh equation:

$$
\mathrm{I}_{\mathrm{S}} \mathrm{I}=\left(\pi^{2} \sin ^{2} \theta\right) / \lambda^{4} \mathrm{r}^{2} \iint \delta \varepsilon\left(\mathrm{r}_{1}\right) \delta \varepsilon\left(\mathrm{r}_{2}\right) \mathrm{dv}_{1} \mathrm{dv}_{2}
$$

Where $\theta$ is the angle between the polarization of the field and the scattered direction and $r$ is the distance from the scattered point, $I_{s}$ is the scattered intensity and $I$ is the incident intensity. Integrating this equation over all angles of a sphere, the total power scattered can be calculated as [55-58]:

$$
\mathrm{P}_{\mathrm{s}}=\mathrm{I}\left(8 \pi^{2} / 3 \lambda^{4}\right) \iint \delta \varepsilon\left(\mathrm{r}_{1}\right) \delta \varepsilon\left(\mathrm{r}_{2}\right) \mathrm{dv}_{1} \mathrm{dv}_{2}
$$

However this equation doesn't include the effects due to TIR (Total Internal Reflection) which causes the whispering gallery modes. Hence the internal beams which reflect from the microsphere surface making an angle greater than the critical angle $\gamma_{0}=$ $\arcsin (1 / \mathrm{n})$, will be consumed by the WGMs, and will be re-circulated in the microsphere. Whereas the beams making an angle smaller than the critical angle will be added to scattering losses and the scattering angles for the TE modes can be denoted as:

$$
\operatorname{Sin}^{2} \gamma_{\mathrm{TE}}=(\mathrm{a}-\mathrm{d} / \mathrm{a})^{2}\left(1-\sin ^{2} \theta \cos ^{2} \theta\right)<1 / \mathrm{n}^{2}
$$

Here $d$ is the distance of the dipole from the surface and a is the radius of the microsphere. For $\mathrm{d}<<\mathrm{a}$ the reflected beams will be consumed into the whispering gallery modes. For $\mathrm{d} \sim \mathrm{a}$, the beams will be used in the scattering and absorption losses. By using thermodynamical calculations the attenuation coefficient due to internal losses can be derived as [55-58]: 


$$
\alpha_{\text {int }}=\left(8 \pi^{3} / 3 \lambda^{4}\right) n^{8} p^{2} k \text { T } \beta_{T}
$$

Here $\mathrm{k}$ is boltzman constant, $\mathrm{T}$ is the glass transition temperature $(368.15 \mathrm{~K})$ for polystyrene, $\beta_{\mathrm{T}}$ is the isothermic compressibility $\left(\sim 2 \times 10^{-10} \mathrm{~m}^{2} / \mathrm{N}\right)$, and $\mathrm{p}$ is the coefficient of photo-elasticity $\left(\sim 2 \times 10^{-12} \mathrm{~m}^{2} / \mathrm{N}\right)$. Hence the $\mathrm{Q}$ factor due to internal scattering can be calculated as:

$$
\mathrm{Q}_{\text {int }}=\mathrm{K}_{\mathrm{TE}}\left(2 \pi \mathrm{n} / \alpha_{\mathrm{int}} \lambda\right)
$$

The suppression coefficient $\mathrm{K}_{\mathrm{TE}}$ describes the relation of complete scattered power to power scattered on satisfying angle conditions. Using numerical calculations the value of $\mathrm{K}_{\mathrm{TE}}$ for polystyrene $(\mathrm{n}=1.5)$ is around 3 .

\section{2-3-2-2 Microsphere Surface Radiation Losses}

We will analytically derive the attenuation coefficient of surface scattering losses of the traveling wave per unit length. Only surface inhomogeneities will be taken into account in this case. We will start with the same expression as in the previous section and integrate it over angles with account of TIR. For the surface dipoles the part of the light scattered outside the sphere will be consumed into radiation modes and hence the suppression coefficient will be [55-58]:

$$
2 \mathrm{~K}_{\mathrm{TE}} /\left(\mathrm{K}_{\mathrm{TE}}+1\right)
$$

Assuming a traveling wave with intensity distribution I (y, z) along the guided surface of the microsphere, the surface roughness will cause inhomogeneities of the dielectric permittivity:

$$
\delta \varepsilon(\mathrm{x}, \mathrm{y}, \mathrm{z})=\left(\varepsilon_{\mathrm{o}}-1\right) \mathrm{f}(\mathrm{x}, \mathrm{y}) \delta \mathrm{z}
$$

$\delta \mathrm{z}$ is the delta function related to surface roughness. Similar to the section 1 the autocorrelation theory can be used to account for the surface roughness variations. 
Assuming that the surface roughness is weakly correlated in case for the microspheres and their correlation function drops to zero at a scale smaller than the wavelength, then the roughness can be described as the standard deviation, $\sigma=\sqrt{ }\left(<\mathrm{f}(\mathrm{x}, \mathrm{y})^{2}>\right)$ and the correlation length as B. Hence the scattered power can be derived as:

$$
\begin{aligned}
& P_{s}=\int I(y, 0)\left(16 \pi^{2} / 3 \lambda^{4}\right)\left(n^{2}-1\right) \pi B^{2} \sigma^{2} d x \\
& P_{s}=P \alpha_{e x t} d x
\end{aligned}
$$

$\mathrm{P}_{\mathrm{s}}$ is the scattered power and $\mathrm{P}=\left(\int \mathrm{I}(\mathrm{y}, \mathrm{z}) \mathrm{dy} \mathrm{dz}\right)$ is the incident power. Assuming that the wave is traveling closer to the surface, the external attenuation coefficient can be derived as:

$$
\alpha_{\mathrm{ext}}=\left(\mathrm{I}(\mathrm{y}, 0) / \int \mathrm{I}(\mathrm{y}, \mathrm{z}) \mathrm{dz}\right)\left(16 \pi^{2} / 3 \lambda^{4}\right)\left(\mathrm{n}^{2}-1\right) \pi \mathrm{B}^{2} \sigma^{2} \quad 2.52
$$

Assuming that only the propagation of TE modes effects the $\mathrm{Q}$ factors, the ratio of the intensities in the equation above can be solved in terms of square of electric fields, and by solving the Bessel function we get, a $\left(n^{2}-1\right) / 2 n^{2}$, where a is the radius of the sphere and $\mathrm{n}$ is refractive index [55-58].

$$
\alpha_{\mathrm{ext}}=\mathrm{a}\left(\mathrm{n}^{2}-1\right) / 2 \mathrm{n}^{2}\left(16 \pi^{2} / 3 \lambda^{4}\right)\left(\mathrm{n}^{2}-1\right) \pi \mathrm{B}^{2} \sigma^{2}
$$

Finally the external quality factor is:

$$
\mathrm{Q}_{\mathrm{ext}}=\left(\mathrm{K}_{\mathrm{TE}} / \mathrm{K}_{\mathrm{TE}}+1\right)\left(3 \lambda^{3} \mathrm{a} / 8 \mathrm{n} \pi^{2} \mathrm{~B}^{2} \sigma^{2}\right)
$$

From the literature, the estimated values of the correlation length of polystyrene microsphere is $B=5 \mathrm{~nm}$ and surface roughness $\sigma=1.5 \mathrm{~nm}$. $\lambda$ is the vacuum wavelength and assuming the microspheres are present in water $(n=1.333)$ medium above the waveguide surface, the total sum of losses in a waveguide-microsphere coupled system is:

$$
\alpha_{\text {total }}=\alpha_{\mathrm{wg}}+\alpha_{\mathrm{int}}+\alpha_{\mathrm{ext}}
$$

The above result is based on a single microsphere waveguide system, and the sum 
of equations (1.100, 3.9, and 3.16). To calculate the results of scattering loss due to a certain microsphere surface coverage on a fixed waveguide surface area, the internal and external microsphere losses will be multiplied by a surface coverage factor $\mathrm{S}$.

$$
\mathrm{S}=(\text { No. of Microspheres Adsorbed/ Saturated }
$$

Adsorption No. in a fixed area) $\times 100$

$$
\alpha_{\text {total }}=\alpha_{\mathrm{wg}}+\mathrm{S}\left(\alpha_{\mathrm{int}}+\alpha_{\mathrm{ext}}\right)
$$

The total losses in a waveguide microsphere coupled system can be estimated and is helpful for characterization of output optical intensity noise and sensitivity.

\section{2-3-3 TE Modal Solutions}

Considering a light wave of frequency $\boldsymbol{\omega}$ being coupled into the whispering gallery modes of a dielectric microsphere of radius a, via an evanescent field of a planar waveguide. The first order and second order spherical modes can be shown as the field [59]:

$$
\mathbf{E}=\alpha_{1}(t) \mathbf{E}_{\omega}(r) e^{-i \omega t}+\alpha_{2}(t) \mathbf{E}_{2 \omega}(r) e^{-2 i \omega t}
$$

Where, $\left|\alpha_{i}\right|^{2}$ is the radiated power and the spherical solution of Maxwell's equations can be represented as:

$$
\begin{aligned}
& \nabla^{2} E-\frac{1}{c^{2}} \frac{\partial^{2} E}{\partial t^{2}}=\mu_{o} \frac{\partial^{2}}{\partial t^{2}}\left[P+\delta(r-a) P_{N L}\right] \\
& \nabla^{2} H-\frac{1}{c^{2}} \frac{\partial^{2} H}{\partial t^{2}}=-\nabla \times \frac{\partial}{\partial t}\left[P+\delta(r-a) P_{N L}\right] \\
& \nabla . E=0, \nabla . H=0
\end{aligned}
$$

Where, $\mathrm{P}$ is the linear polarization and $\mathrm{P}_{\mathrm{NL}}$ is a surface non-linearity. 


$$
r \cdot\left(\nabla^{2} E\right)=\nabla^{2}(r \cdot E)-2 \nabla \cdot E=\nabla^{2}(r . E)
$$

This gives,

$$
\begin{aligned}
& \left(\nabla^{2}-\frac{1}{c^{2}} \frac{\partial^{2}}{\partial t^{2}}\right)(r . E)=\mu_{o} r \cdot \frac{\partial^{2}}{\partial t^{2}}\left[P+\delta(r-a) P_{N L}\right] \\
& \left(\nabla^{2}-\frac{1}{c^{2}} \frac{\partial^{2}}{\partial t^{2}}\right)(r . H)=-r \cdot\left(\nabla \times \frac{\partial}{\partial t}\left[P+\delta(r-a) P_{N L}\right]\right)
\end{aligned}
$$

The above equations have solutions of form $Y_{\operatorname{lm}}(\theta, \square) Z_{1}(r) e^{-i \omega l p t}$, where $Y_{\operatorname{lm}}$ is a spherical harmonic and $Z_{1}$ is spherical Bessel function [60]. The indices $1, \mathrm{~m}$ and $\mathrm{p}$ are orbital, azimuthal and radial numbers respectively. For TE mode solution [60]:

$$
r . H=\sum_{l m p} \sqrt{\frac{2 l(l+1)}{Z_{O}}} \alpha_{l m p}^{T E}(t) Z_{l}(r) Y_{l m}(\theta, \varphi) e^{-i \omega l p t}
$$

Where, $Z_{\mathrm{o}}=\left(\sqrt{ } \mu_{\mathrm{o}} / \varepsilon_{\mathrm{o}}\right)$ is the vacuum impedance and $\left|\alpha_{l m p}\right|^{2}$ is the total radiated power. Using orthogonality of spherical harmonics [59]:

$$
\frac{1}{r} \frac{d^{2}}{d r^{2}}\left(r Z_{l}\right)+\left[n^{2} K_{L P}^{2}-\frac{L(L+1)}{r^{2}}\right] Z_{l}=-\delta(r-a) \frac{J^{T E}}{\alpha}
$$

Where $\mathrm{K}_{\mathrm{LP}}=\omega_{\mathrm{LP}} / \mathrm{c}, \mathrm{n}=\mathrm{n}\left(\omega_{\mathrm{LP}}\right)$ is the index of refraction and:

$$
J^{T E}=\sqrt{\frac{Z_{o}}{2 L(L+1)}} \iint Y_{l m} r .\left(\nabla \times \frac{\partial P_{N L}}{\partial t}\right) \times e^{i \omega L P} \sin \theta d \theta d \varphi
$$

For $\mathrm{r} \neq \mathrm{a}$, the solution of the above equation represents the spherical Bessel function:

$$
b_{l}(x)=\sqrt{\frac{2 \pi}{x}} B_{l+1 / 2}(x)
$$

We find: 


$$
F_{E}\left(K_{L P} a\right)=\frac{J^{T E}}{K_{L P} \alpha}
$$

Which is the characteristic equation for a $\mathrm{TE}$ mode when $\mathrm{J}^{\mathrm{TE}}=0$. Therefore the $\mathrm{TE}$ mode equation can be solved to $[59,60]$ :

$$
\frac{d \alpha}{d t}+\Gamma_{L P} \alpha \approx \frac{c J^{T E}}{i K_{L P} a G_{E}\left(K_{L P} a\right)}
$$

Where $\Gamma_{L P}$ is the decay rate of the TE mode. And:

$$
G_{E}(x)=\frac{d F_{E}(x)}{d x}
$$

Is the derivative of the function $\mathrm{F}_{\mathrm{E}}(\mathrm{x})$.

\section{2-4 Analytical Model for Microdisk Modes}

\section{2-4-1 Microdisk Resonators}

The resonant Whispering gallery modes in a Microdisk are similar to those of a Microsphere and hence constructive interference takes place after every roundtrip and a phase shift of the order of $2 \pi$. The TE modes in a Microsphere are determined by the transverse dimensions of the looped waveguide but on the other hand, modes can have more than one intensity maxima in the radial or vertical direction in a Microdisk and are known as Radial or Vertical Cavity modes. The figure below shows three different modes for a disk resonator. 

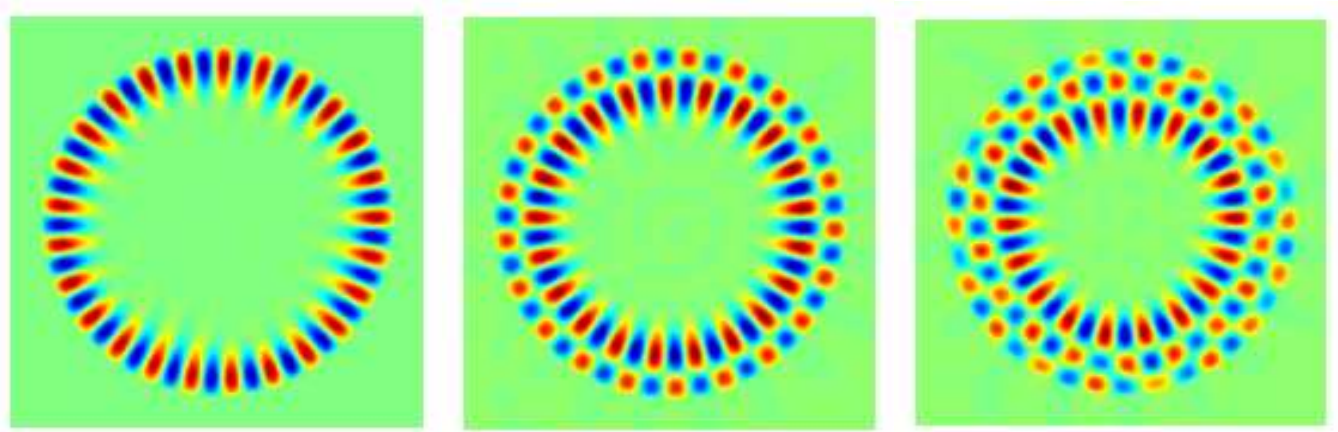

Fig 19 Whispering Gallery Modes in a Microdisk with different radial mode numbers [61]

The modes can have three radial intensity maxima and are known as whispering gallery modes. Hence three or more different modes can be found in a disk having same mode number $\boldsymbol{m}$ but have a different resonant wavelength. The dips in the transmission spectra correspond to these resonant modes.

\section{2-4-2 Scalar Potentials}

The vector problem involving scalar potentials can be used to find the modes in a Microdisk. Assuming a homogeneous medium containing no free charge or current, the Maxwell's equations can be written as [62]:

$$
\begin{aligned}
& \nabla \times E=-\mu_{o} \frac{\partial H}{\partial t} \\
& \nabla \times H=\varepsilon_{r} \varepsilon_{o} \frac{\partial E}{\partial t} \\
& \nabla . E=0 \\
& \nabla . H=0
\end{aligned}
$$

The two vector potentials $\mathbf{F}$ and $\mathbf{G}$ can be created as [62]: 


$$
\begin{aligned}
& \nabla \times F=H \\
& \nabla \times G=E
\end{aligned}
$$

Both fields are without divergence, and $\mathrm{F}$ forms the magnetic vector potential:

$$
E=-\mu_{o} \frac{\partial F}{\partial t}+\frac{1}{\varepsilon_{r} \varepsilon_{o}} \int \nabla(\nabla . F) d t(\nabla \times G)
$$

Assuming that $\mathrm{F}$ has harmonic time dependence $\mathrm{e}^{-\mathrm{i} \omega \mathrm{t}}$ :

$$
\left(\nabla^{2}+k^{2}\right) F=0
$$

Where $k^{2}=\varepsilon_{r} \omega^{2} / c^{2}$

$$
\begin{aligned}
& H=\varepsilon_{r} \varepsilon_{o} \frac{\partial G}{\partial t}-\frac{1}{\mu_{o}} \int \nabla(\nabla \cdot G) d t(\nabla \times F) \\
& \left(\nabla^{2}+k^{2}\right) G=0
\end{aligned}
$$

These vector potentials can be used to represent the two independent polarizations:

$$
\mathbf{F}=f z \text { and } \mathbf{G}=g z
$$

Where $f$ and $g$ are scalar potentials, and it becomes easier to solve the Helmholtz equation in cylindrical coordinates [62]:

$$
\begin{aligned}
& H_{z}=k^{2} g+\frac{\partial^{2} g}{\partial z^{2}} \quad E_{z}=i \omega \mu_{o}\left(f+\frac{1}{k^{2}} \frac{\partial^{2} f}{\partial z^{2}}\right) k_{o} \\
& H_{\rho}=\frac{\partial^{2} g}{\partial z \partial \rho}+\frac{k_{o}}{\rho} \frac{\partial f}{\partial \theta} \quad E_{\rho}=i \omega \mu_{o}\left(\frac{k_{o}}{k^{2}} \frac{\partial^{2} f}{\partial \rho \partial z}+\frac{\partial g}{\rho \partial \theta}\right)
\end{aligned}
$$




$$
H_{\theta}=\frac{\partial^{2} g}{\rho \partial z \partial \theta}-k_{o} \frac{\partial f}{\partial \rho} \quad E_{\theta}=i \omega \mu_{o}\left(\frac{k_{o}}{k^{2}} \frac{\partial^{2} f}{\partial \rho \partial z}+\frac{\partial g}{\partial \rho}\right)
$$

$\mathrm{H}_{z}$ depends only on $g$ and $\mathrm{E}_{\mathrm{z}}$ depends only on $\mathrm{f}$, therefore modes with $\mathrm{f}=0$ are $\mathrm{TE}$ and modes with $\mathrm{g}=0$ are $\mathrm{TM}$.

\section{2-4-3 Microdisk Whispering Gallery Modes}

The cylindrical coordinates Helmholtz equation can be shown to be in the form:

$$
\nabla_{T}^{2} f+\frac{\partial^{2} f}{\partial z^{2}}+k^{2} f=0
$$

Where $\nabla_{T}^{2}$ is called the transverse Laplacian in cylindrical coordinates. By separating $f$ into $f_{T}$, and depending upon $\rho, \varphi, f_{z}$, and $z$, we can reduce the three dimensional Microdisk problem into a two dimensional one [62]:

$$
\frac{\partial^{2} f_{z}}{\partial z^{2}}+\beta^{2} f_{z}=0 \quad \nabla_{T}^{2} f_{T}+T^{2} f_{T}=0
$$

Where $k^{2}=T^{2}+\beta^{2}$ and by allowing $\beta$ to be real or imaginary, $\mathrm{f}_{\mathrm{z}}$ can have a form of oscillating and decaying solutions, inside and outside of the Microdisk:

$$
f_{\phi}=C e^{i m \phi} \quad \text { and } \quad f_{\rho}=A_{m} J_{m}(T \rho)+B_{m} N_{m}(T \rho)
$$

In the above equation $\mathrm{J}_{\mathrm{m}}$ is a Bessel function and $\mathrm{N}_{\mathrm{m}}$ is a Neumann function of the order of $\boldsymbol{m}$ and the general solution of $\mathrm{F}$ takes the form [62]:

$$
f(z, \varphi, \rho)=f_{z}(z) f_{\phi}(\phi) f_{\rho}(\rho)
$$




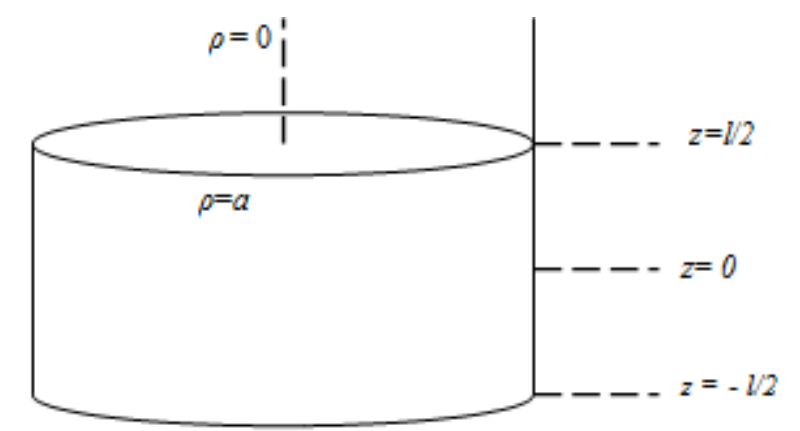

Fig 20 Geometry and Coordinates of Microdisk

Assuming the boundaries of the Microdisk to be perfect magnetic and having zero conductivity, with the tangential components of $\mathbf{H}$ and normal components of $\mathbf{E}$ to be zero across the boundaries [63]. This will yield a closed form solution of mode frequencies and field profiles. As $\mathrm{B}_{\mathrm{m}}=0$ :

$$
\begin{aligned}
& f(z, \varphi, \rho)=A_{m} J_{m}(T \rho) \cos (m \phi) \cos (\beta z+\theta), \\
& \text { for } \quad|z|<l / 2, \rho<a
\end{aligned}
$$

As the field is zero outside the disk:

$$
T=\frac{y_{m n}}{a} \quad \text { and } \quad \beta=\frac{p \pi}{l}
$$

Where $y_{m n}$ is the nth root of the first derivative of the mth Bessel function and $\mathrm{p}$ is an integer. We know that $k^{2}=\omega^{2} \mu_{o} \varepsilon_{r} \varepsilon_{o}=T^{2}+\beta^{2}$, therefore the mode wavelengths are given by [63]: 


$$
\lambda_{m n p}=\frac{2 \pi \sqrt{\varepsilon_{r}}}{\sqrt{\left(\frac{p \pi}{l}\right)^{2}+\left(\frac{y_{m n}}{a}\right)^{2}}}
$$

The equation above represents the whispering gallery modes in a Microdisk resonator. The integers $\mathrm{m}, \mathrm{n}$, and $\mathrm{p}$ are azimuthal, radial and vertical $(\mathrm{z})$ mode numbers respectively.

\section{2-4-4 Effective Index of Refraction}

As we solved the Microdisk mode problem from three dimensions to two dimensions, the effective index of refraction can be utilized to understand the propagation of modes in transverse directions $[62,63]$. The true and effective index of refraction can be defined as:

$$
\begin{aligned}
& n=\frac{k}{k_{o}} \text { where, } k_{o}=\frac{\omega}{c} \\
& \bar{n}=\frac{T}{k_{o}}
\end{aligned}
$$

Assuming the disk lies in the air $\left(\varepsilon_{\mathrm{r}}=1\right)$ and the electromagnetic wave attenuates outside the disk evanescently. So that for $|z|>l / 2$ and $\rho<a$

$$
f_{z}=D e^{-\alpha(|z|-l / 2)}
$$

Assuming that only first order fundamental modes are formed, so $\mathrm{p}=1$, applying boundary conditions $[62,63]$ : 


$$
n^{2} \sqrt{\bar{n}^{2}-1}=\sqrt{n^{2}-\bar{n}^{2}} \tan \left(\frac{l}{2} k_{o} \sqrt{n^{2}-\bar{n}^{2}}\right)
$$

The above equation represents the effective index of refraction for a Microdisk and is dependent on $\boldsymbol{k}$ and therefore the wavelength of light $\lambda$.

\section{2-4-5 Micro-Disk Vertical Cavity Modes}

The vertical cavity modes are formed in a Microdisk in the vertical (z) direction along the height of the disk. The vertical cavity modes are based on the Fabry-Perot optical resonator into which a source medium (active or passive) is inserted. Generally the cavity is formed between two highly reflective surfaces separated by the cavity length $\boldsymbol{L}$. The resonance wavelength of the cavity is determined by the phase condition requiring that the phase of the traveling wave after one round trip is a multiple of $2 \pi$.

$$
2 k L=m 2 \pi \quad \text { where, } \quad k=2 \pi \bar{n} / \lambda
$$

Here, $\mathrm{m}$ is an integer; $\mathrm{k}$ is the wave propagation constant or wave number. And $\bar{n}$ is the effective refractive index of the disk. At a certain cavity length $L$ an optical thickness of $\bar{n} L$ is a multiple of $\lambda / 2$.

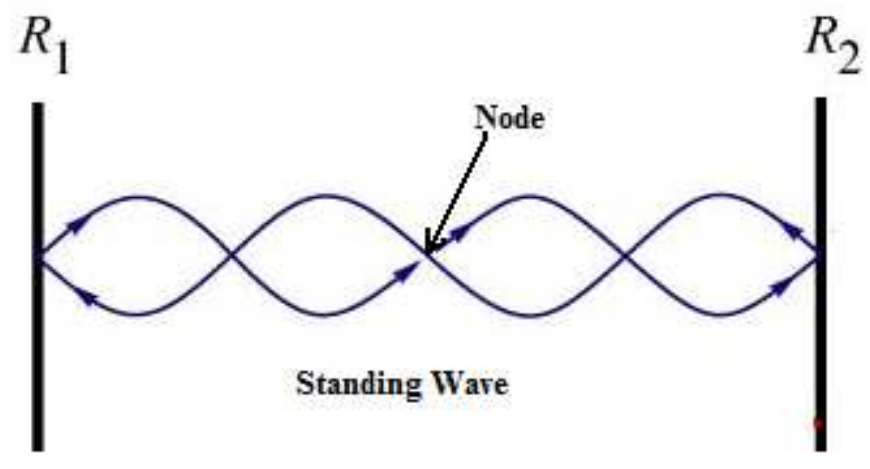

Fig 21 Standing wave pattern formed in an optical cavity 
The optical source (or a quantum well in case of an LED) is placed at an anti-node of the electromagnetic field (standing wave pattern) in order to provide maximum optical enhancement. This puts an extreme demand on the accuracy of the layer thicknesses used in the cavity, as a small displacement can cause a shift of the standing wave peak resulting in a reduction of optical enhancement. The enhancement factor can be denoted as [63]:

$$
\Gamma_{e n h}=1+\frac{\sin \left(2 \pi \bar{n} L_{a} / \lambda\right)}{2 \pi \bar{n} L_{a} / \lambda} \cos \left(4 \pi \bar{n} d_{o} / \lambda\right)
$$

Here $d_{o}$ is the displacement of the standing wave peak from the active region (optical source placement) in the cavity. For the maximum enhancement, we get the relation:

$$
2 \mathbf{L}=(\mathbf{m} \lambda) / \overline{\mathbf{n}}
$$

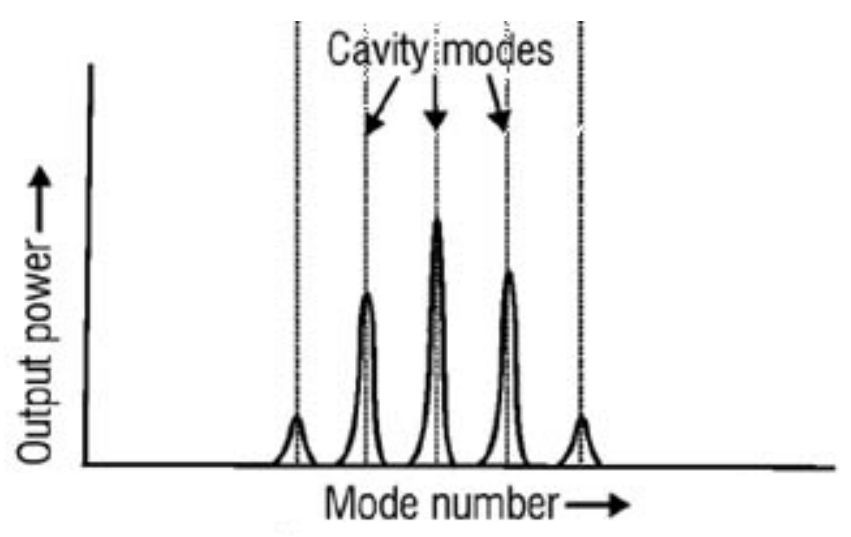

Fig 22 Resonant modes of an optical cavity [64]

The above relation forms the longitudinal modes of an optical cavity. A long cavity sustains more resonant modes spaced more closely. Ideally the cavity length is formed to be the order of few wavelengths which results in a large longitudinal mode spacing, implicating that only one or two longitudinal cavity mode overlaps with the source medium, which improves the optical enhancement. 


\section{2-5 Opti-Wave Simulations}

\section{2-5-1 Microcavity Resonators as Passive Components}

The main applications of passive microcavity resonators are wavelength filtering, optical switching, routing, modulation and multiplexing. The factors which decide the efficient working of a passive microcavity resonator are:

1. Free spectral range (Mode spacing between adjacent resonances).

2. Quality factor (The ratio of the energy stored to the energy dissipated per round trip).

3. Finesse (The ratio of the 'free spectral range' to the width of the resonance).

4. Extinction ratio (The ratio of optical transmission at on-resonance to offresonance transmission)

The conditions for an ideal resonator are wide free spectral range, higher Q, large finesse to accommodate many resonance channels, lower optical losses and a high extinction ratio. To achieve a FSR (free spectral range) on the order of tens of nanometers, the size of the resonator should be kept to few micrometers. High quality etching creates a smoother sidewall profile and scattering losses can be kept to a minimum in order to achieve a high finesse. These microcavity resonators can be integrated with other photonics devices to form complex integrated circuits. Optiwave BPM and FDTD simulations can be used to design microcavity resonators for optimal performance, which is discussed next.

\section{2-5-2 Beam Propagation Method (Opti-BPM)}

Analytical solutions of wave equations can be formed easily for simple structures where a complete understanding of the optical modes of a waveguide is present. But for a more complex optical structure where a complicated solution of mode coupling is involved 
a numerical analysis is performed. Various kinds of beam propagation methods (BPMs) have been developed such as fast Fourier transform (FFT-BPM), finite difference (FDBPM) and finite element (FE-BPM). The FFT-BPM has many disadvantages such as long computational times, inability to use simple transparent boundary conditions, and inadequacy in handling large index differences. Incorporating transparent boundary conditions and use of Padé approximant operators for FD-BPM has made it possible for it to be used for design of optical waveguides made of high-contrast index materials [65].

The beam propagation method involves the analysis of optical modes, where it decomposes a spatial mode into a superposition of a number of plane waves traveling in different directions. The tool then integrates the propagation of these waves into one spatial mode to analyze diffraction effects caused due to an inhomogeneous medium. It takes into account the phase shift encountered at every reflection/refraction at a boundary of different indices. Thus, the medium in which the wave is traveling is modeled as a sequence of lenses separated by short sections of homogenous space.
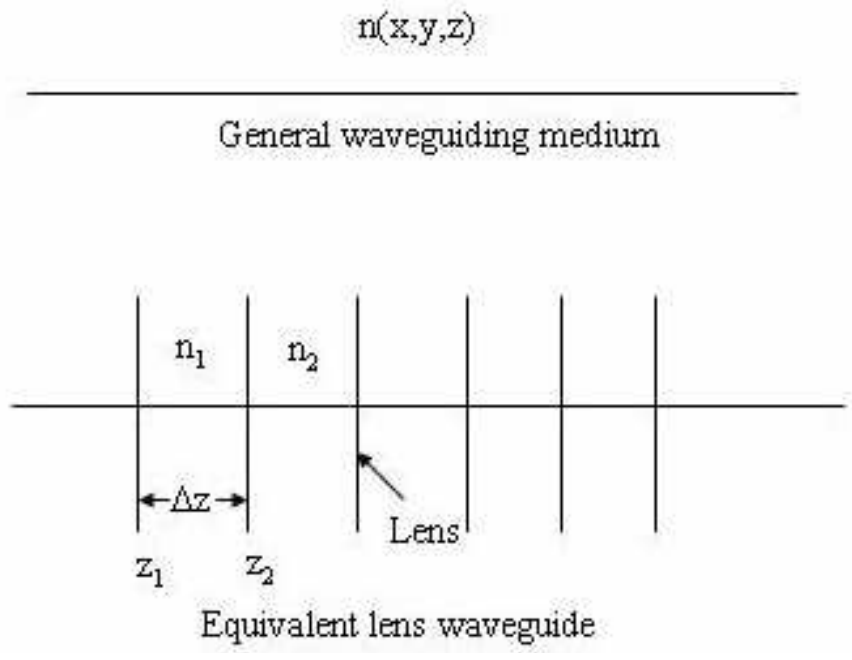

Fig 23 Optical path divided into a series of lenses in Opti-BPM software [66]

The finite difference-BPM involves starting with the wave equation and dividing the field into a slowly varying envelope function and a very fast phase term. The wave 
equation for the y-directed field is $[65,66]$ :

$$
\frac{\partial^{2} E_{y}}{\partial z^{2}}+\frac{\partial^{2} E_{y}}{\partial x^{2}}+k_{o}^{2} \varepsilon_{r} E_{y}=0
$$

The electric field is divided using the slowly varying envelope approximation:

$$
E_{y}(x, y, z)=\phi(x, y, z) \exp (-j \beta z)
$$

Substitution into the wave equation $[65,66]$ :

$$
2 j \beta \frac{\partial \phi}{\partial z}-\frac{\partial^{2} \phi}{\partial z^{2}}=\frac{\partial^{2} \phi}{\partial x^{2}}+k_{o}^{2}\left(\varepsilon_{r}-n_{e f f}^{2}\right) \phi
$$

When it is assumed that $\frac{\partial^{2} \phi}{\partial z^{2}}=0$, equation 3.65 is reduced to the Fresnel wave equation or paraxial wave equation. Generally this approximation works well and is the simplest and fastest solver. However wide angle analysis using Padé approximant operators is used when there is large refractive index contrast. A paraxial case is where the field polarization is almost parallel to the direction of propagation and off axial effective index is similar to the axial value. However when the beam is diverging as it propagates, the propagation constant becomes a strong function of off-axial effective index placing an upper limit on how wide a beam may diverge in BPM [65]. Paraxial approximation leads to errors for angles less than 20 degrees whereas the $(1,1)$ Padé approximant operator is accurate up to 30 degrees. Higher order approximants lead to higher accuracy at large angles but also increase the numerical complexity and hence the computation times [66]. Overall, the BPM is one of the most widely used methods for planar waveguide and direction coupler waveguide simulations and analysis of electromagnetic modes. For a more complicated structure case of a curved, spherical or cylindrical waveguide Opti-FDTD is used as discussed in the next section. 


\section{2-5-3 Finite Difference Time Domain Method (Opti-FDTD)}

The finite difference time domain method (FDTD) was first introduced by Kane Yee [67] and became one of the most popular methods to simulate electromagnetic wave interactions with dielectric structures. The basic idea is to discretize space-time into blocks, where Maxwell's equations can be changed from partial differential equations to difference equations, which are evaluated at every point in the space-time grid to advance the fields temporally. FDTD is different from finite element method (FEM) as it is a time-domain method and not based on steady state solutions.

\section{2-5-3-1 Difference Equations}

Considering the curl equations of Faraday's law and Ampere's law and taking their divergence results in [67]:

$$
\begin{aligned}
& \frac{\partial}{\partial t}(\nabla . \mathbf{B})=0 \\
& (\nabla . \mathbf{D})=\rho_{f}
\end{aligned}
$$

The above equation is the Poisson's equation. In a homogeneous region of Cartesian coordinates the Faraday's law and Ampere's law become:

$$
\begin{gathered}
\mu \frac{\partial H_{x}}{\partial t}=\frac{\partial E_{y}}{\partial z}-\frac{\partial E_{z}}{\partial y} \\
\varepsilon \frac{\partial E_{x}}{\partial t}=\frac{\partial H_{z}}{\partial y}-\frac{\partial H_{y}}{\partial z}
\end{gathered}
$$

The above equations can be broken into difference equations, by discretizing spacetime steps: 


$$
\left.\frac{d F(x)}{d x}\right|_{x=x_{o}}=\frac{f\left(x_{o}+\Delta x / 2\right)-f\left(x_{o}-\Delta x / 2\right)}{\Delta x}
$$

Here $\Delta x$ is the step size in the $\mathrm{x}$ direction. The numerical error is reduced by computing derivatives at points between those on which the functions are defined [68]. Therefore the $\mathrm{E}_{\mathrm{x}}$ and $\mathrm{H}_{\mathrm{y}}$ components are also defined on a half integer grid as shown in figure below.

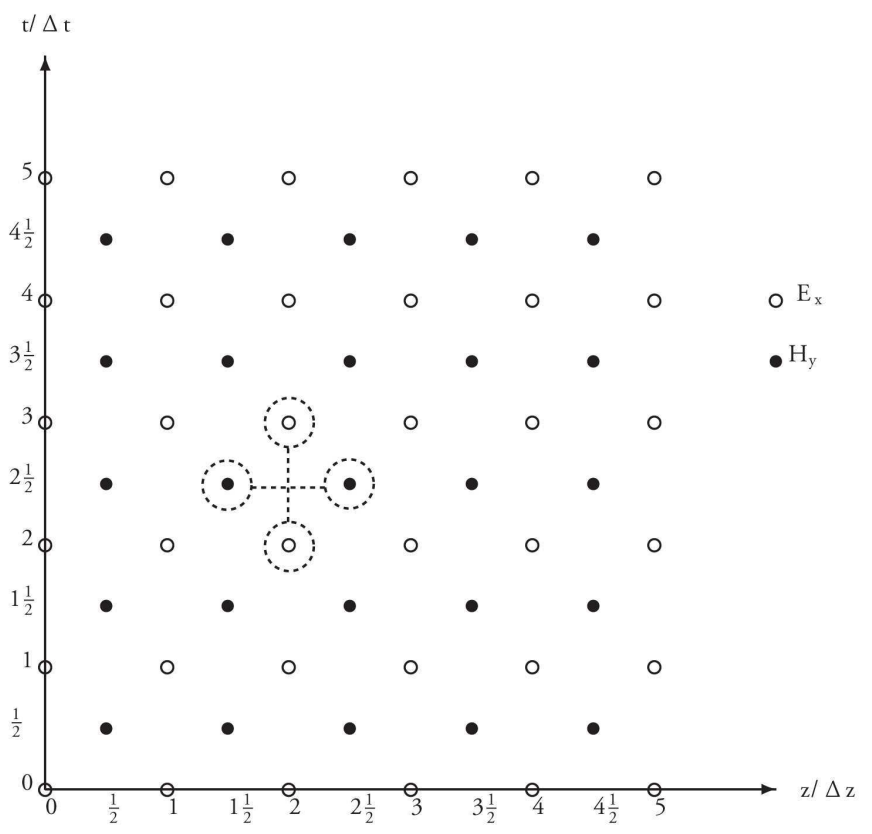

Fig $24 \mathrm{E}_{\mathrm{x}}$ and $\mathrm{H}_{\mathrm{y}}$ defined in a half-integer staggered grid [68]

The two dimensional grid can be modified into a three dimensional using a Yee cell shown in figure 25. The $\mathrm{H}$ components are located on the center of cube faces and $\mathrm{E}$ components are located on the center of cube edges. The time step used to separate $\mathrm{E}$ and $\mathrm{H}$ is $\Delta \mathrm{t} / 2$ or half time step. 


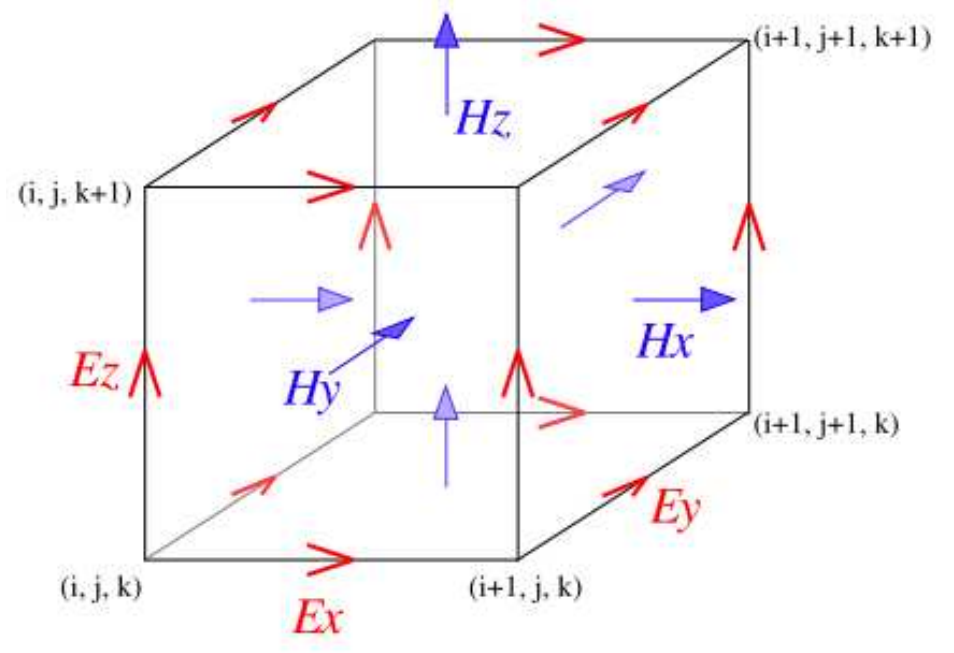

Fig 25 Yee Cell showing staggered grid in three dimensions [69]

Now the set of difference equations can be derived for the 3D case:

$$
\begin{aligned}
& \varepsilon \frac{\left.E_{x}\right|_{i+\frac{1}{2}, j, k} ^{n+1}-\left.E_{x}\right|_{i+\frac{1}{2}, j, k} ^{n}}{\Delta t} \\
& =\frac{\left.H_{z}\right|_{i+\frac{1}{2}, j+\frac{1}{2}, k} ^{n+\frac{1}{2}}-\left.H_{z}\right|_{i+\frac{1}{2}, j-\frac{1}{2}, k} ^{n+\frac{1}{2}}}{\Delta y}-\frac{\left.H_{y}\right|_{i+\frac{1}{2}, j, k+\frac{1}{2}} ^{n+\frac{1}{2}}-\left.H_{y}\right|_{i+\frac{1}{2}, j, k-\frac{1}{2}} ^{n+\frac{1}{2}}}{\Delta z}
\end{aligned}
$$

Similar equations for other components can be derived and this equation can be solved for $E_{x}$ at time $t=(n+1) \Delta t$ in terms of the fields at times $t=n \Delta t$ and $t=(n+1 / 2) \Delta t$. This leads to the advancement of fields in time and space. The wave propagation in the $\mathrm{z}$ direction with the $\mathrm{x}-\mathrm{z}$ plane used for $2 \mathrm{D}$ simulations and a perpendicular $\mathrm{E}_{\mathrm{y}}$ component is introduced in the $3 \mathrm{D}$ case. The TE components considered for our simulations are $\mathrm{H}_{\mathrm{x}}, \mathrm{E}_{\mathrm{y}}$ and $\mathrm{H}_{\mathrm{z}}$. 


\section{2-5-3-2 Sources of Error}

The discretization of space-time can also introduce errors in the simulated data. The most common error is the "stair-stepping" when curved surfaces are modeled using a rectilinear Yee cell grid. These errors can be compared to the surface roughness on the sidewalls of the disk, which might help the simulation to match the fabrication error. A grid based on Cartesian coordinates would create error modeling a cylindrical or hexagonal structure, which can be avoided by using an adaptive mesh of higher density and smaller grid size $[68,69]$.

Another form of error is the numerical dispersion resulting from relative sizes of time and space steps. In one dimension the time step is denoted as $\Delta \mathrm{t}=\Delta \mathrm{x} / \mathrm{c}$ [68], but in multiple dimensions the wave need not travel precisely along the grid axes. This leads to a numerical anisotropy in the phase velocity, with waves traveling faster along the grid diagonals as compared to grid axis, which can cause a phase lag [70]. These effects can be reduced by increasing the number of mesh cells relative to the wavelength, but a very high density grid will increase practical challenges or RAM processing and longer simulation times. Ideally the grid density can be designed relative to wavelength [71]:

$$
\max (\Delta x, \Delta y, \Delta z) \leq \frac{\lambda_{\min }}{10 n_{\max }}
$$

And the time step should follow the condition [71]:

$$
\Delta t \leq \frac{n}{c \sqrt{\frac{1}{(\Delta x)^{2}}+\frac{1}{(\Delta y)^{2}}+\frac{1}{(\Delta z)^{2}}}}
$$

Both of these errors play a significant role in whispering gallery modes as the path of light is continually turning, which leads to unphysical artifacts in the simulated data. Properly designed and taking into account the error factors, FDTD can be efficiently used to model electromagnetic behavior in dielectric structures. 


\section{2-5-3-3 Description of Simulations}

The Optiwave Opti-FDTD software is used in this study for simulation of whispering gallery and vertical cavity modes in microsphere and microdisk structures. Firstly the value of permittivity and refractive index is defined at every point on the grid. A specialized boundary condition is used known as anisotropic perfectly matched layers (PML). These layers have pre-defined conductivity tensors which cause the wave to attenuate with minimal reflection as it leaves the simulation volume [71]. Electromagnetic radiation in form of a Gaussian-modulated continuous wave (GMCW) in space and time is introduced as either a plane or spherical wave which has the form [71]:

$$
E_{n}(t)=A e^{-\frac{1}{2}\left(\frac{t-t_{o}}{W}\right)^{2}} \sin (\omega t)
$$

Where $\mathrm{n}=(\mathrm{x}, \mathrm{y}, \mathrm{z})$, leading to a Gaussian distribution of frequencies centered at $\omega$. The fields can be sampled using observation points, lines and areas. The observation points collect the time-domain data which is converted to frequency domain by DFT (Discreet Fourier Transform) and plotted on a wavelength scale for analysis. 


\section{Chapter 3 \\ Experiment and Results}

\section{3-1 SPARROW Biosensor}

\section{3-1-1 Planar Waveguide Simulations}

The Optiwave (BPM) Beam Propagation Method provides an efficient way for modal analysis and light propagation in planar waveguides. The mode solver tool is used to model propagation constants, waveguide thickness, optical mode profile and effective refractive indices of a single and coupled planar waveguides. These parameters help in understanding the electromagnetic behavior and optimizing the waveguides before the fabrication process.

Before designing of the SPARROW coupled waveguide structure, a planar waveguide was simulated to analyze the TE modes, and mode profile variation with changes in cladding index and waveguide thickness. To simply the approach, TE modes were studied in our case, and TM modes were not used for our device operation and sensitivity analysis of evanescent waves. Incident radiation from a Helium-Neon laser beam ( $\lambda=632.8 \mathrm{~nm}$ ) was used for passive excitation of waveguides in modeling as well as experimental analysis. Asymmetric and symmetric waveguide profiles were simulated for an alumina waveguide of thickness $1 \mu \mathrm{m}$ and refractive index of 1.636. The TE mode profile for a cover index of $n=1$ and $n=1.6$ was studied as shown in the figure below. 

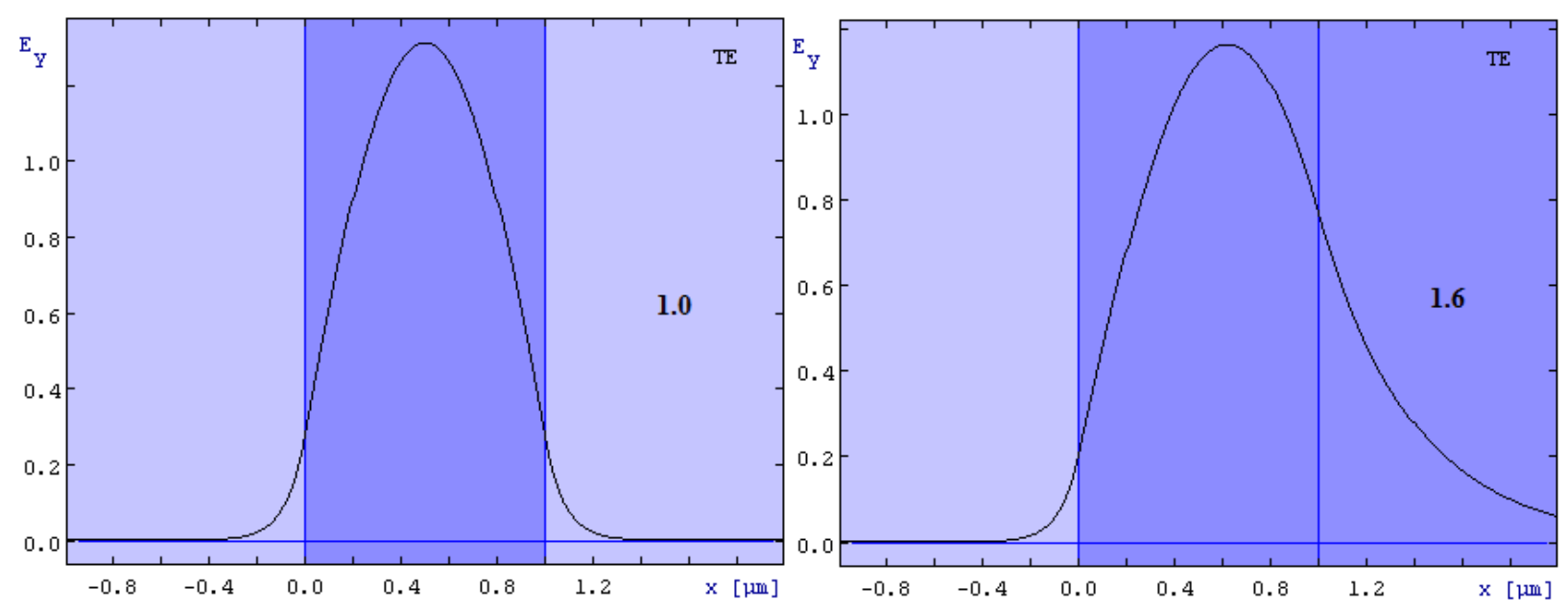

Fig 26 Single TE Mode ( $E_{y}$ field) for Symmetric and Asymmetric Waveguides

The evanescent field extends into the cover medium as the refractive index is increased from $n=1$ to $n=1.6$. Also, in the figure below the thickness of waveguide is reduced to $0.5 \mu \mathrm{m}$ and the TE mode profile is distorted.
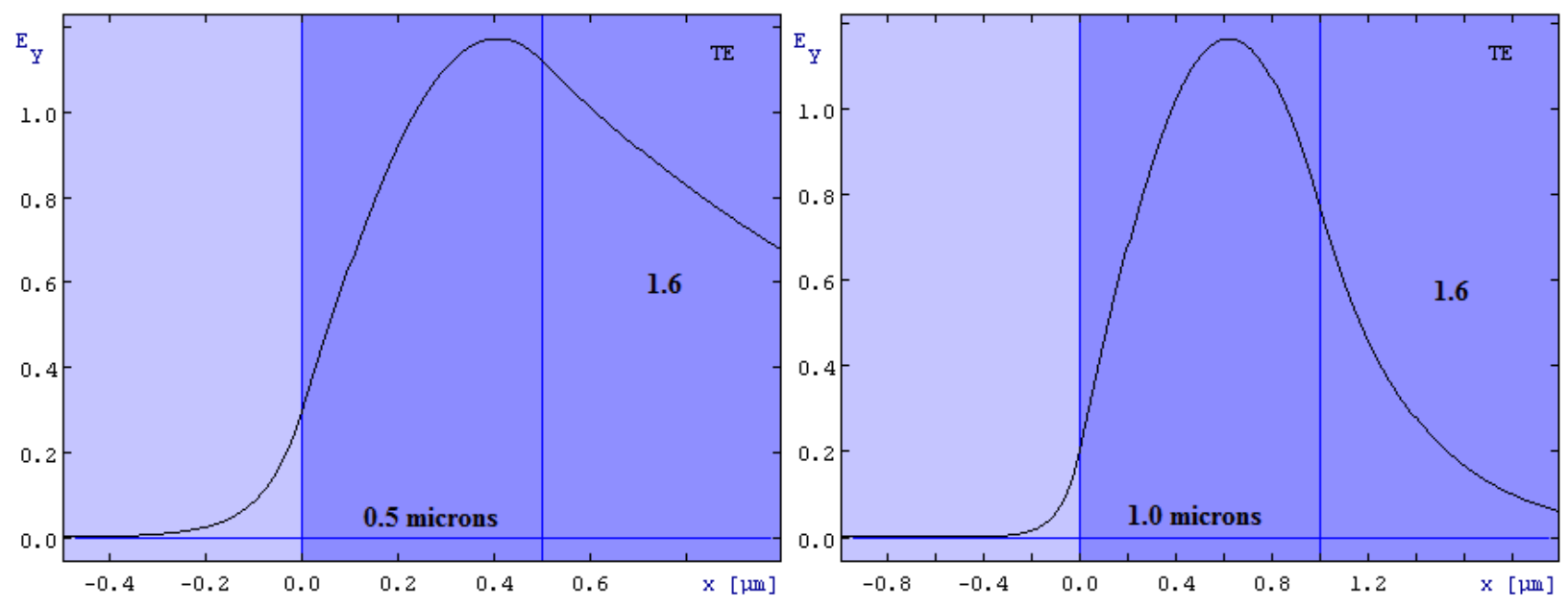

Fig 27 TE Mode ( $E_{y}$ field) for Asymmetric Waveguides with Different Thicknesses

Appropriately designed, the planar waveguide thickness and index of cladding can be modeled to achieve the desired TE mode profile. In next section, a coupled waveguide structure (SPARROW) can be discussed in detailed. 


\section{3-1-2 Coupled Waveguide (SPARROW) Simulations}

The Stack Planar Affinity Regulated Resonant Optical Waveguide (SPARROW) device is a directional coupler, with two planar waveguides when placed together exchange power periodically between the two guides. Opti-BPM tool was used to simulate TE modes, coupling length and power transfer of waveguides. Two aluminum oxide waveguides $\left(\mathrm{Al}_{2} \mathrm{O}_{3}\right)$ with index of 1.636 and separated by a silicon dioxide layer $\left(\mathrm{SiO}_{2}\right)$ of index 1.46 were designed to have matched propagation constants. Borofloat glass of index 1.4701 was used as the substrate and the coupled waveguide structure was simulated for air and water as the superstrate medium. The designed waveguides with matched propagation constants, $\beta_{\mathrm{m} 1}=\beta_{\mathrm{m} 2}($ where, $\beta=$ n.k.cos $\theta)$ were calculated to have a top guide thickness of $200 \mathrm{~nm}$ and bottom guide thickness of $142 \mathrm{~nm}$, with water (1.333) as cover medium index.

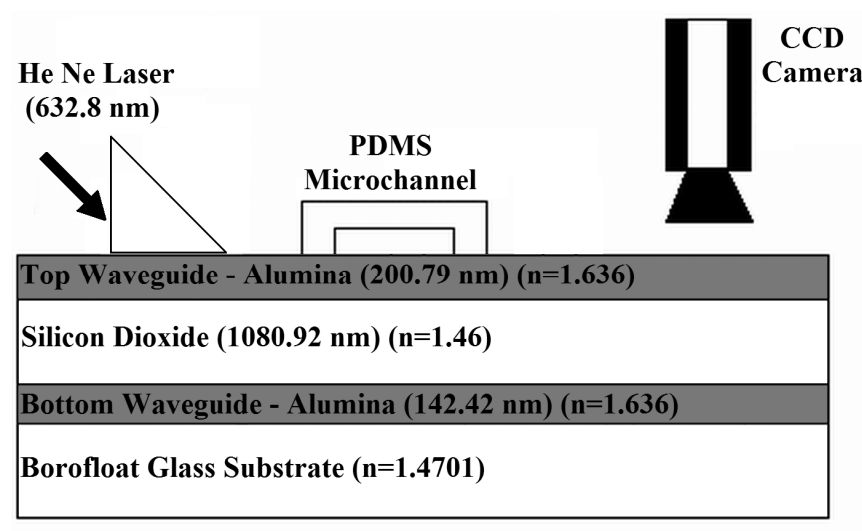

Fig 28 Designed Coupled Waveguide for Water Superstrate

Helium-Neon laser light is coupled into the top waveguide using a prism coupler, and after some distance the evanescent field from the top waveguide overlaps the mode of the bottom waveguide and couples completely into bottom waveguide. The waveguide indices and thicknesses are designed such that the optical mode propagates into the bottom waveguide for Air/PDMS superstrate, and coupled power transfers for water as superstrate (as shown in figure 29). 


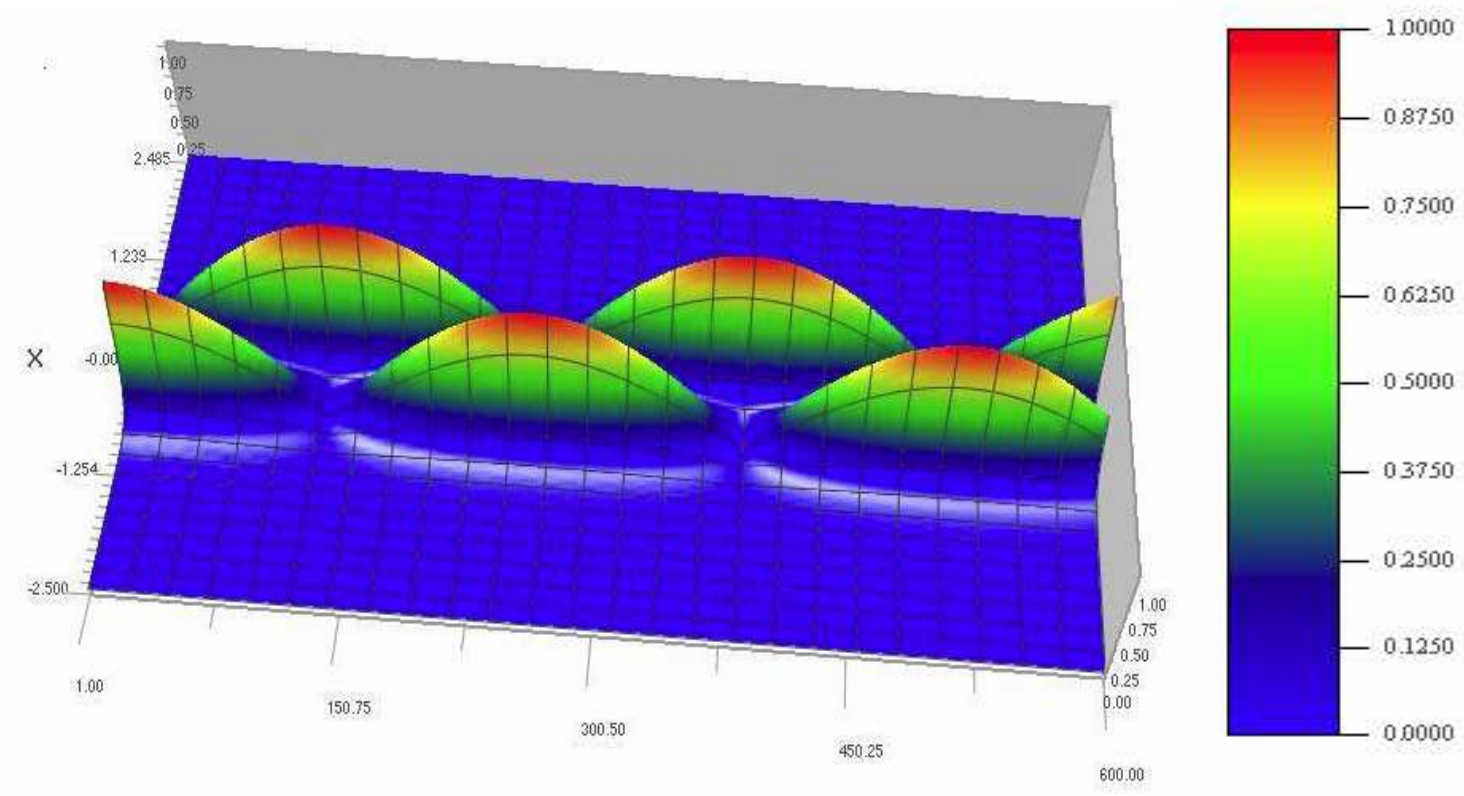

Fig 29 Optical Intensity for Coupled Waveguides

The effective refractive indices of the top and bottom waveguides (for air as superstrate) were simulated as $\mathrm{N}_{\text {eff (top) }}=1.483$ and $\mathrm{N}_{\text {eff (bottom) }}=1.5$. The propagation constants of the individual waveguides (for air as superstrate) were calculated as:

$$
\begin{aligned}
& \beta_{1 \text { (top) }}=\mathrm{N}_{\text {eff (top) }}(2 \pi / \lambda)=14.724 \\
& \beta_{2 \text { (bottom) }}=\mathrm{N}_{\text {eff (bottom) }}(2 \pi / \lambda)=14.893
\end{aligned}
$$




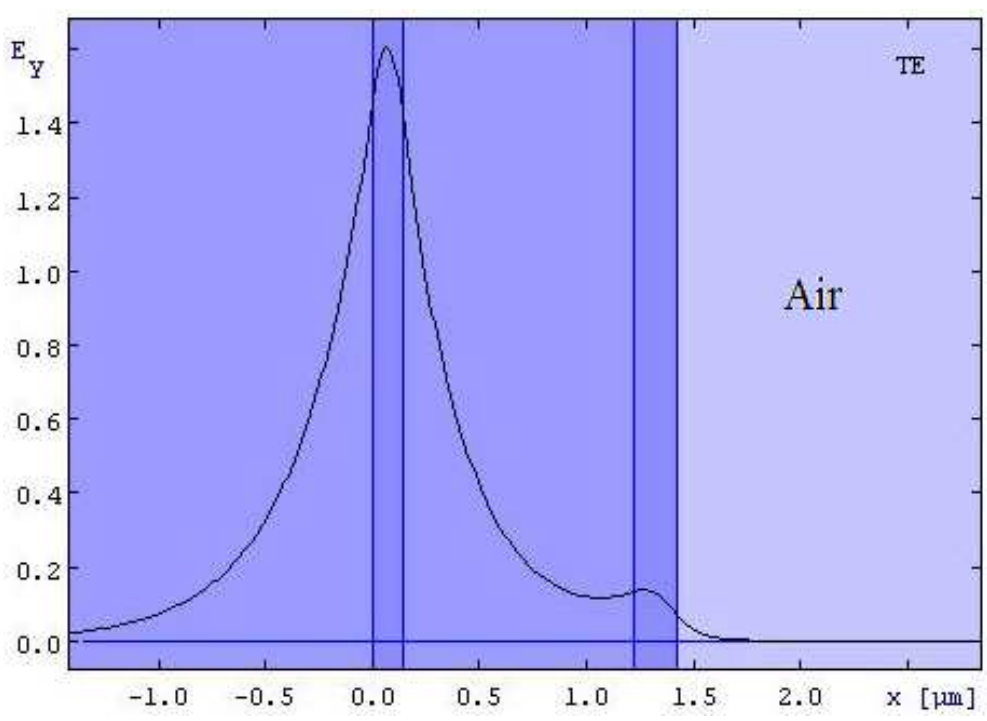

Fig 30 Optical Mode Profile in Un-Coupled Waveguides (Air Superstrate)

The waveguide mode propagates in the bottom waveguide for the designed waveguide structure for Air/PDMS as the superstrate, as there is no coupling between the two waveguides. When water (1.333) is present as superstrate above the top waveguide, the two waveguides are designed to optically resonate, with matched propagation constants, and there is a complete exchange of power between the two waveguides. This complete exchange of power takes place over a distance called Coupling Length $\left(\mathbf{L}_{\mathbf{c}}\right)$.
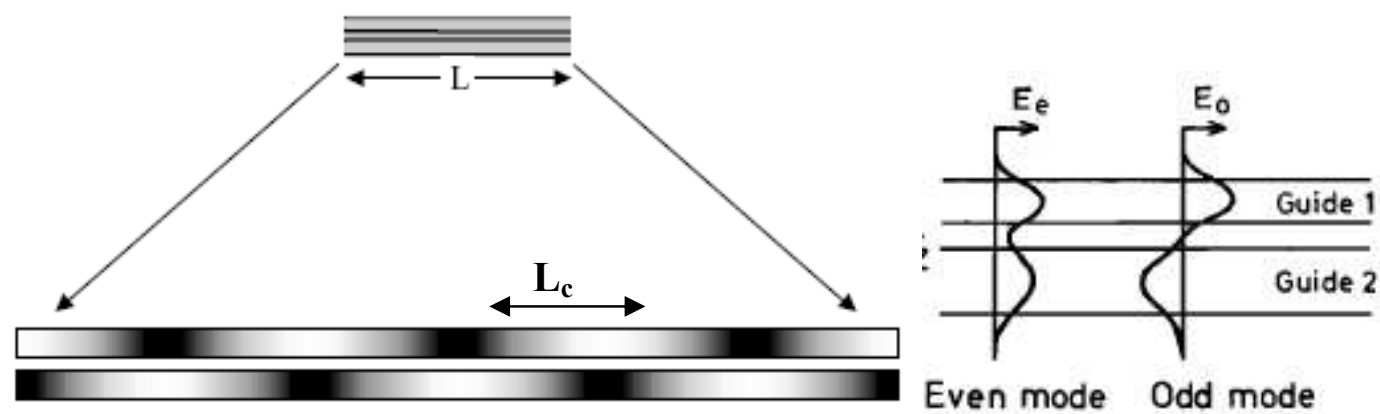

Fig 31 Coupling Length and the even mode 'TE0' and second odd mode 'TE1' [72]

The two waveguides exchange power periodically, back $n$ forth, over the coupling length, and throughout the interaction length ' $\mathrm{L}$ ' of the waveguides, as long as water is the 
superstrate. The fundamental 'even mode (TE0)' and 'odd mode (TE1)' in the simulation plot below shows the tuned/coupled waveguides for water as superstrate.

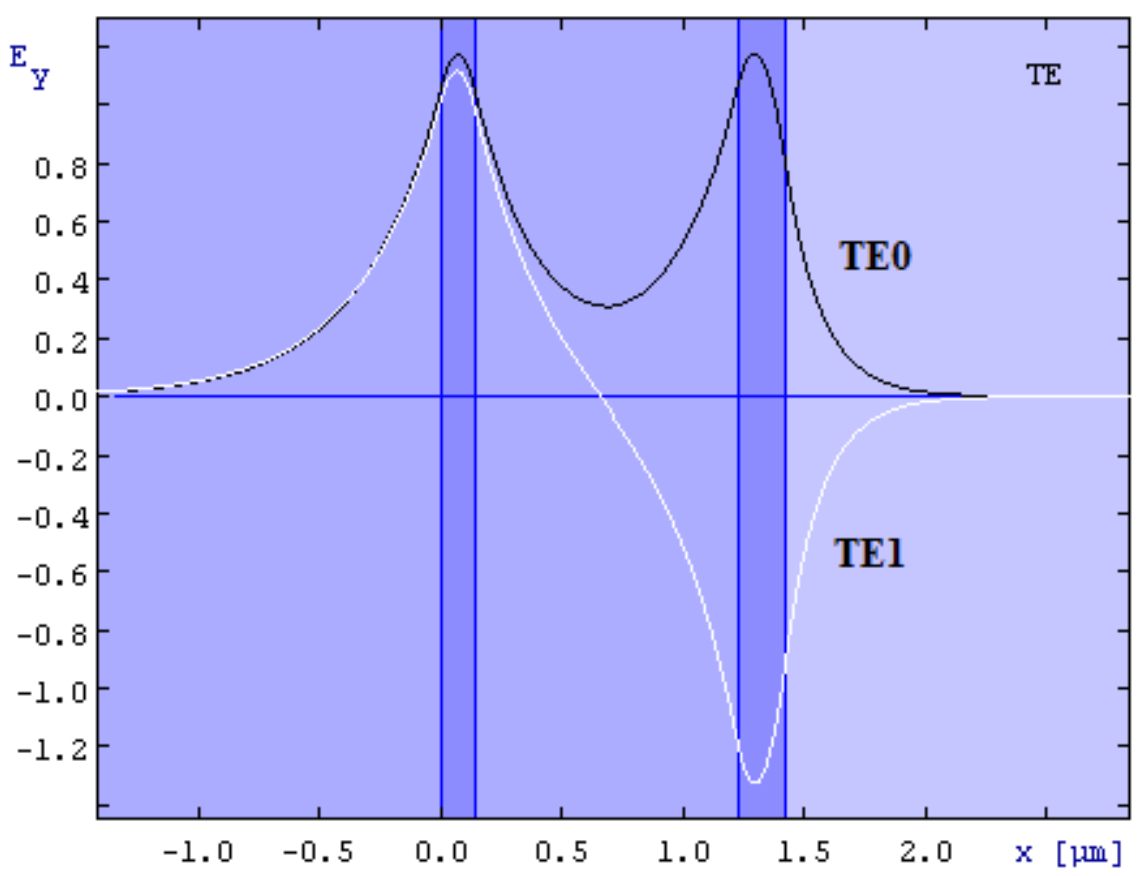

Fig 32 Even Mode (TE0) and Odd Mode (TE1) in Coupled Waveguides (Water Superstrate)

As the power is transferred periodically back $\mathrm{n}$ forth, throughout the interaction length ' $\mathbf{L}$ ', the net power in each waveguide mode is $\sim 50 \%$ of the total power. The OptiBPM simulated effective indices for coupled waveguides with water as superstrate were $\mathrm{N}_{\text {eff }}$ $\left(\right.$ top) $=\mathrm{N}_{\text {eff (bottom) }}=1.5$ and the calculated matched propagation constants were:

$$
\begin{aligned}
& \beta_{1 \text { (top) }}=\mathrm{N}_{\text {eff (top) }}(2 \pi / \lambda)=14.893 \\
& \beta_{2 \text { (bottom) }}=\mathrm{N}_{\text {eff (bottom) }}(2 \pi / \lambda)=14.893
\end{aligned}
$$

For the TE0 and TE1 coupled modes, the propagation constants and Neff were simulated as: 


$$
\begin{array}{llll}
\beta_{\mathrm{e}}=14.906 & \mathrm{n}_{\mathrm{e}}=1.501 & \text { (Even Mode) } & 3.5 \\
\beta_{\mathrm{o}}=14.875 & \mathrm{n}_{\mathrm{o}}=1.498 & \text { (Odd Mode) } & 3.6
\end{array}
$$

Using the above simulated and calculated parameters the coupling length of the waveguides can be calculated as $L c=\pi /(\beta e-\beta o)=101.34 \mu m$ [72].

\section{3-1-3 Biolayer Design and Simulations}

The SPARROW device structure for air/water superstrate was simulated previously, and the coupling parameters and propagation constants were calculated. Based on the coupled waveguides structure, the interaction length of the superstrate medium can be measured, which would carry the biolayer in a PDMS microchannel on the top waveguide surface. As the propagation constants of the two waveguides match for the coupled waveguide structure:

$$
\Delta \beta=\beta 1-\beta 2=0
$$

Initially, the power being coupled to the bottom waveguide for air/PDMS superstrate, the interaction length will be designed to be an even number of coupling lengths $(\sim 1200 \mu \mathrm{m})$ so that the power is transferred back to the bottom waveguide after the channel transit. Due to fabrication uncertainty in the waveguides indices and thicknesses, there will be some power remaining in the top waveguide. The total power in the top waveguide at the end of interaction length can be calculated. Assuming the initial power in the bottom waveguide is $\mathrm{P} 1$, and the power remaining in the top waveguide after the interaction length (L) is P2 [73]:

$$
\begin{aligned}
& P 2=\frac{\operatorname{Sin}^{2}\left\{\left[(k L)^{2}+(\Delta \beta . L / 2)^{2}\right]^{1 / 2}\right\}}{1+(\Delta \beta / 2 k)^{2}} \times P 1 \\
& \mathrm{P} 2=0.0036 \times \mathrm{P} 1
\end{aligned}
$$

Where $\mathrm{k}=0.76$ (simulated coupling coefficient) and $\mathrm{L}=1200 \mu \mathrm{m}$ (Total interaction 
length). Therefore the total power remained in the top waveguide is $0.36 \%$, and $99.64 \%$ of the power is transferred back to the bottom waveguide, after an even number of coupling lengths. The power is exchanged 12 times for a coupling length of $1200 \mu \mathrm{m}$ between the bottom and top waveguide and eventually couples back to the bottom waveguide at the end of the microchannel. The remaining $0.36 \%$ of power in top waveguide is scattered out of the PDMS/Air interface and an additional SU-8 layer.

For the designed interaction length, the biolayer index variations can be simulated to analyze the power variations in the coupled waveguides. As the refractive index of the biolayer superstrate is increased, the coupled power in the top waveguide is increased.

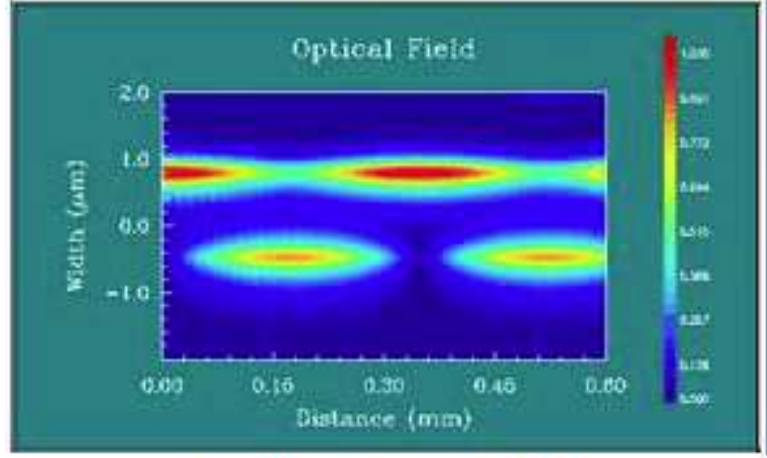

Biolayer index 1.3411

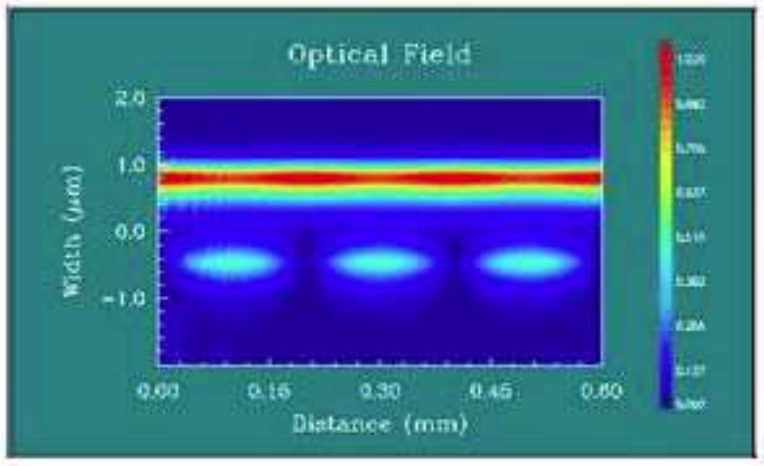

Biolayer index 1.3637

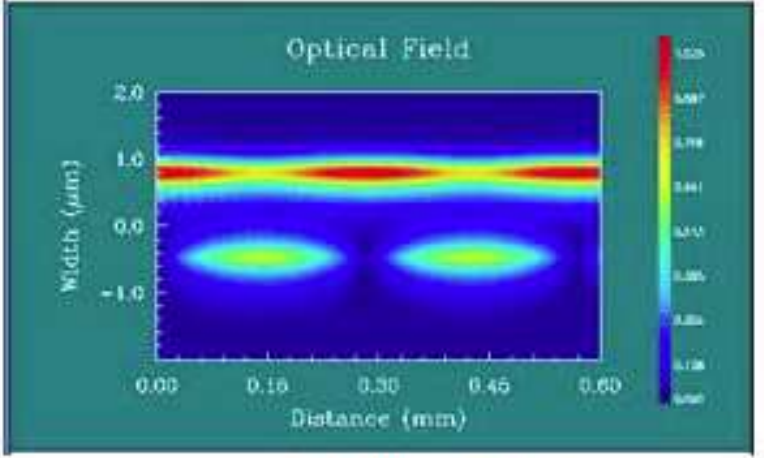

Biolayer index 1.3495

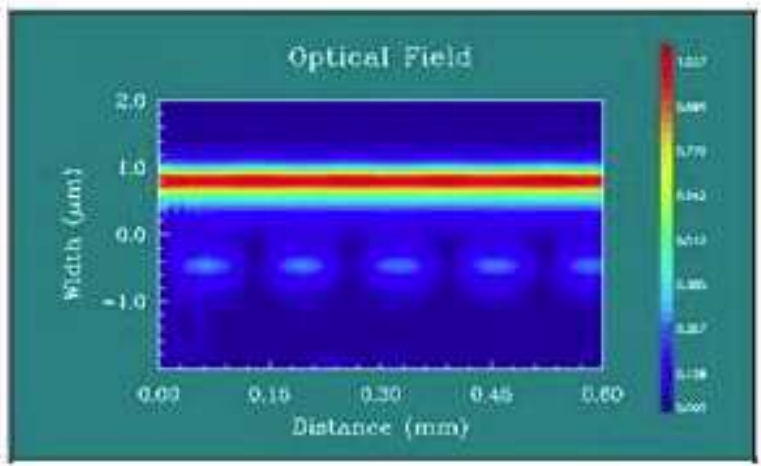

Biolayer index 1.385

Fig 33 Index Variations of Biolayer Superstrate and Coupled Power

Also, the Opti-BPM tool was used to simulate the variation in bottom waveguide power due to changes in thickness and indices of each waveguide and the oxide layer. 

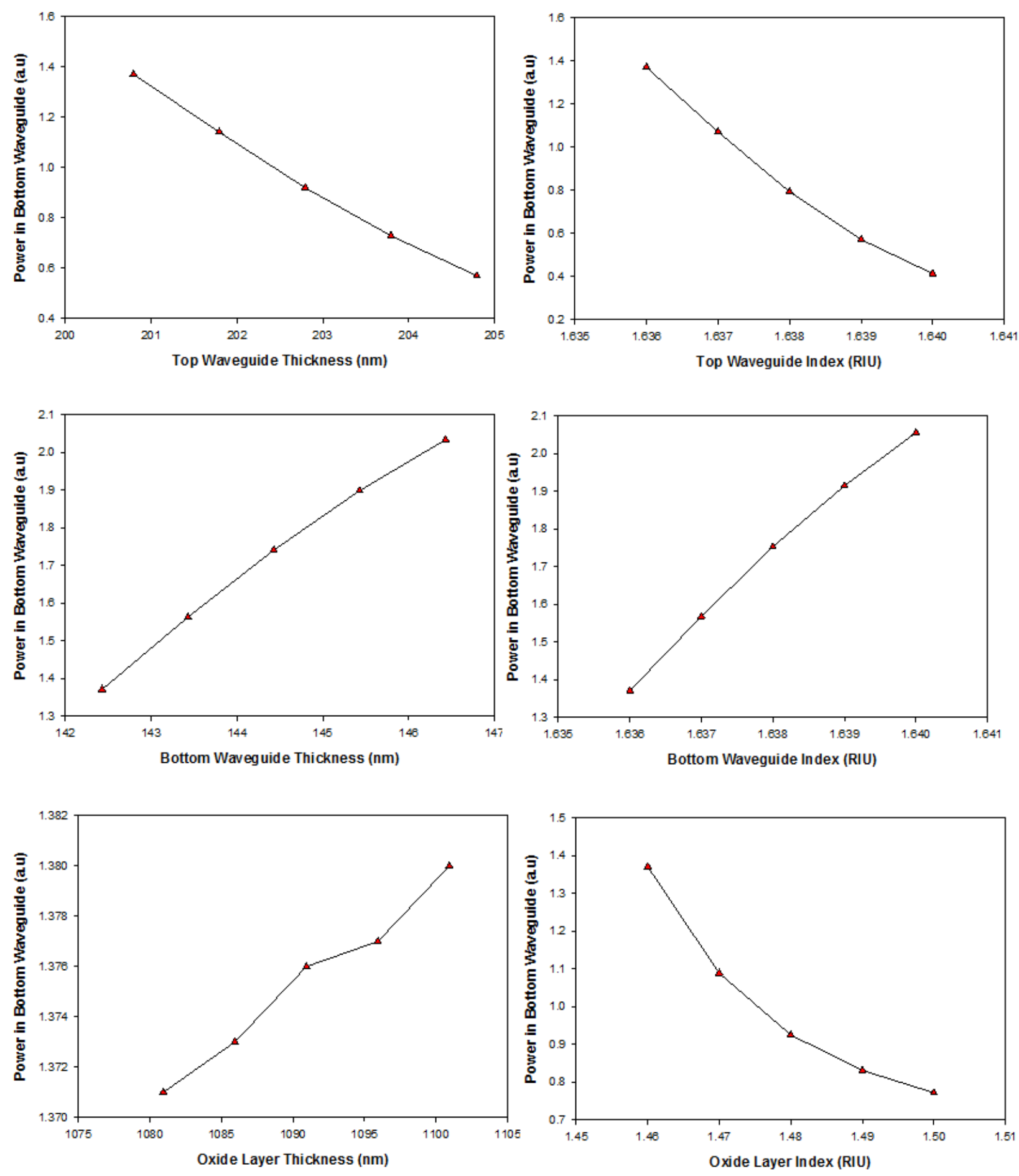

Fig 34 Power in Bottom WG with Changes in Thickness and Index of Layers 
As the changes in the analyte refractive index of the biolayer superstrate are increased, a reduction in the bottom waveguide power is measured. An increase in top waveguide thickness or index resulted in a reduction of bottom waveguide power. Whereas, increasing the thickness or index of bottom waveguide increased the bottom waveguide output power. The overall effect is a sensing mechanism of the changes of refractive indices in the SPARROW structure and the biolayer analyte in the microchannel, which will be shown experimentally in section 3-1-5. Before transducer sensitivity measurements, the coupled waveguide stacked structure was characterized optically and will be discussed in the next section.

\section{3-1-4 Optical Characterization of Alumina Waveguides}

The alumina waveguides were characterized to assure single TE mode operation and low optical propagation loss prior to proceeding with fabrication of a microfluidic channel and evanescent wave sensitivity measurements. A Metricon 2010 Prism Coupler setup was used to couple a polarized $632.8 \mathrm{~nm}$ He-Ne laser to each waveguide's single TE mode using a gadolinium gallium garnet right isosceles prism $(\mathrm{n}=1.965)$. The optical loss was measured by acquiring an image of the waveguide streak using a QImaging Retiga 2000R CCD camera as shown in figure 35 and extraction of the pixel values in the region of interest using MATLAB.

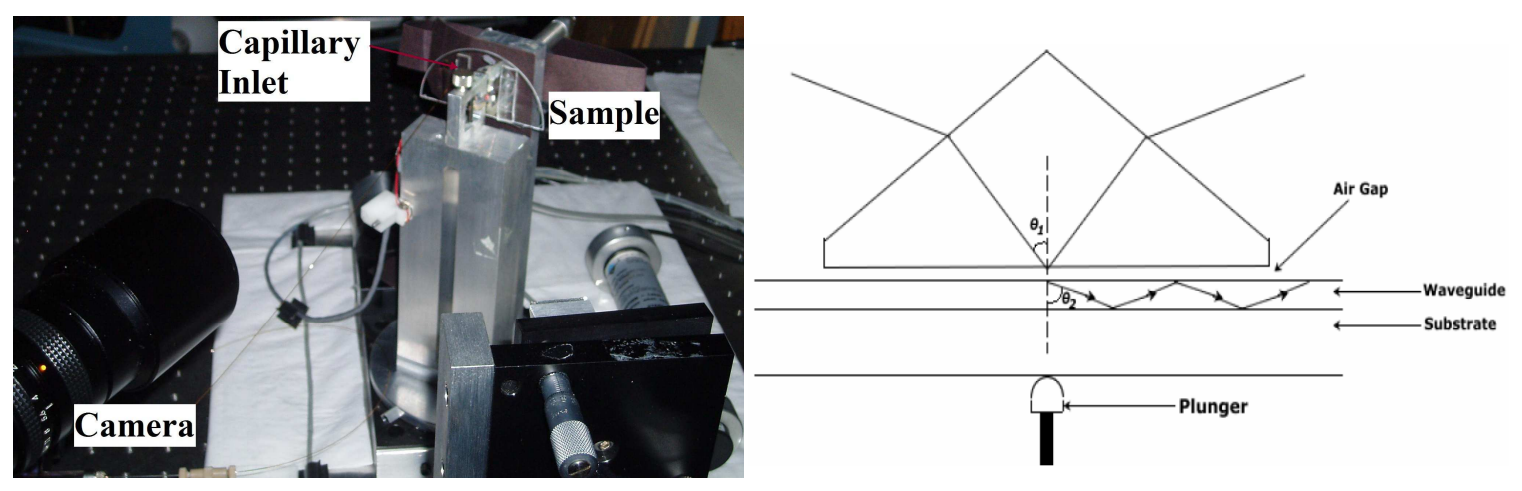

Fig 35 (a) Metricon 2010 Prism Coupler Setup, with Aligned Waveguide Sample and CCD Camera (b) Ray Diagram Showing Coupled Optical Path 
Before characterizing the alumina waveguides for optical loss measurements, the noise in the laser beam and the CCD camera was analyzed. The He-Ne laser beam was warmed up for 3 hours and then the optical power stability was measured using a beam profiler. A $0.15 \%$ error was recorded over 180 minutes. The (QImaging Retiga 2000R) CCD camera images of the waveguide streak were also captured and the net error was measured to be $1.85 \%$. These error values were taken into account for all transducer experiments conducted for analyte sensitivity analysis.
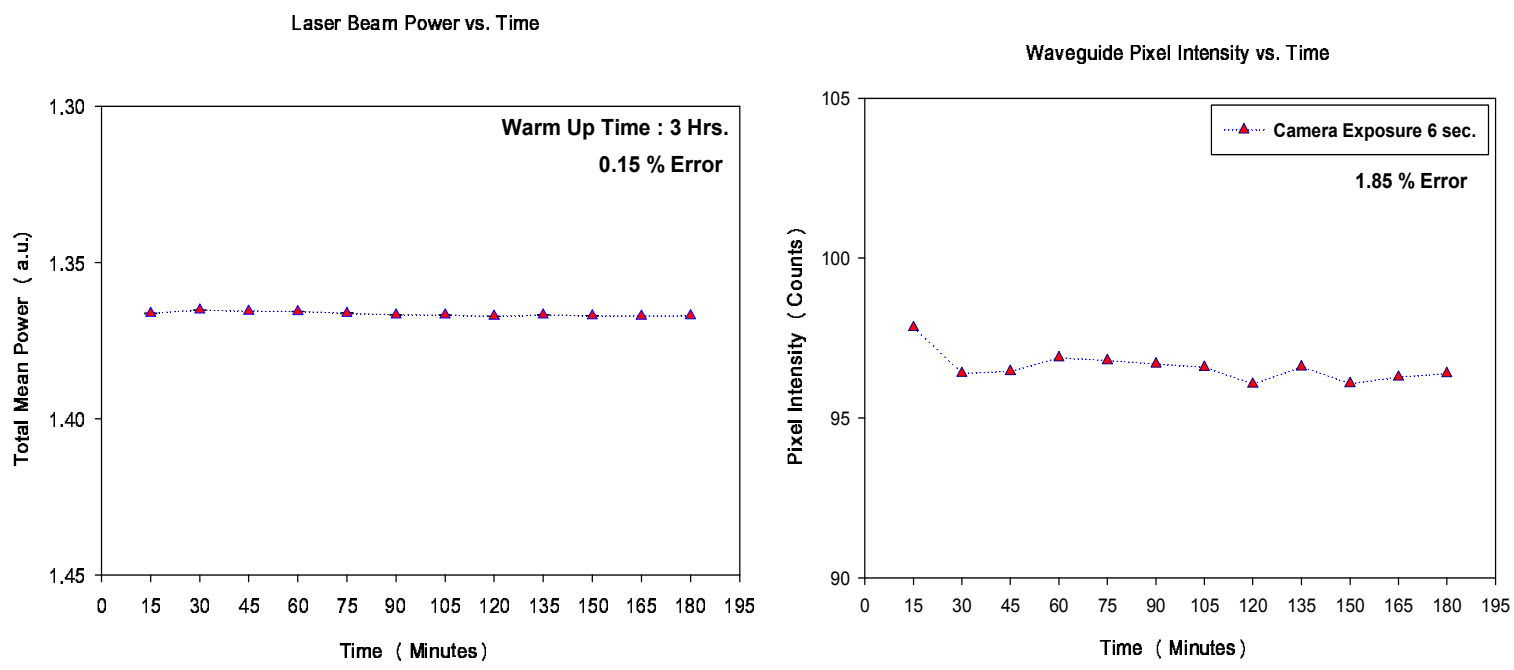

Fig 36 Laser Beam and CCD Camera Noise Characterization

The coupled laser beam propagates into the waveguide and is shown in figure 37. The CCD camera captures a high quality image in the (ROI) region of interest and the MATLAB program is used to read the image and convert into pixel values based on a color scale bar (figure 38 ). 


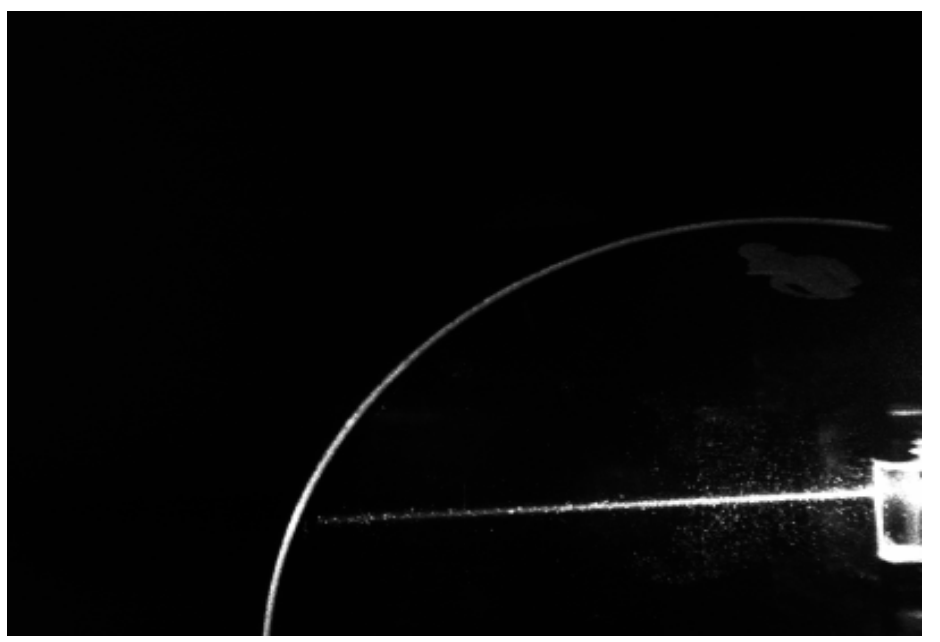

Fig 37 Propagated Laser Power in Alumina Waveguide

The optical intensity decay can be plotted, and the scattering centers were shown due to crystal defects in the waveguide. A linear fit to the logarithmic data plot is then used to calculate the optical loss values. Loss values were determined to be approximately 1 $\mathrm{dB} / \mathrm{cm}$.

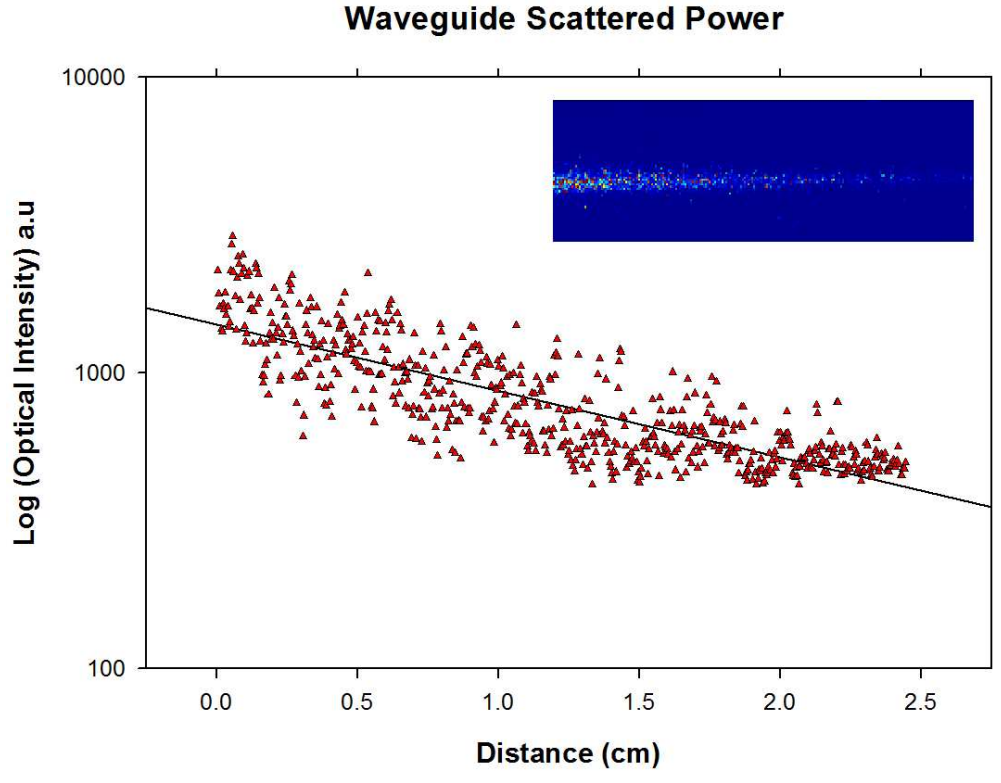

Fig 38 Optical Intensity Decay and MATLAB Image of Waveguide Streak 


\section{3-1-5 Sucrose Experimental Results}

Fiber-optics and planar waveguides based sensors have been reported in literature with advances in the stacked thin film coupled waveguide sensing for a sensitive and accurate detection $[74,75]$. The first part of the experimental approach is to construct and characterize the sensitivity analysis of a SPARROW (Stacked Planar Affinity Regulated Resonant Optical Waveguide) sensor [20]. The evanescent wave detection of various concentrations of aqueous sucrose solution was characterized. The sensing process is based on the measurements of changes in optical power caused by changes in the refractive index due to analyte attachment over the sensor surface. The sensing device was targeted for detection without the requirement of labeling. The analyte mass binding is non-specific and measurements were purely based on the optical absorbance and scattering properties of the molecules. Hence it simplifies the detection by only exposing the analyte to the transducer and measuring the changes in the optical power.

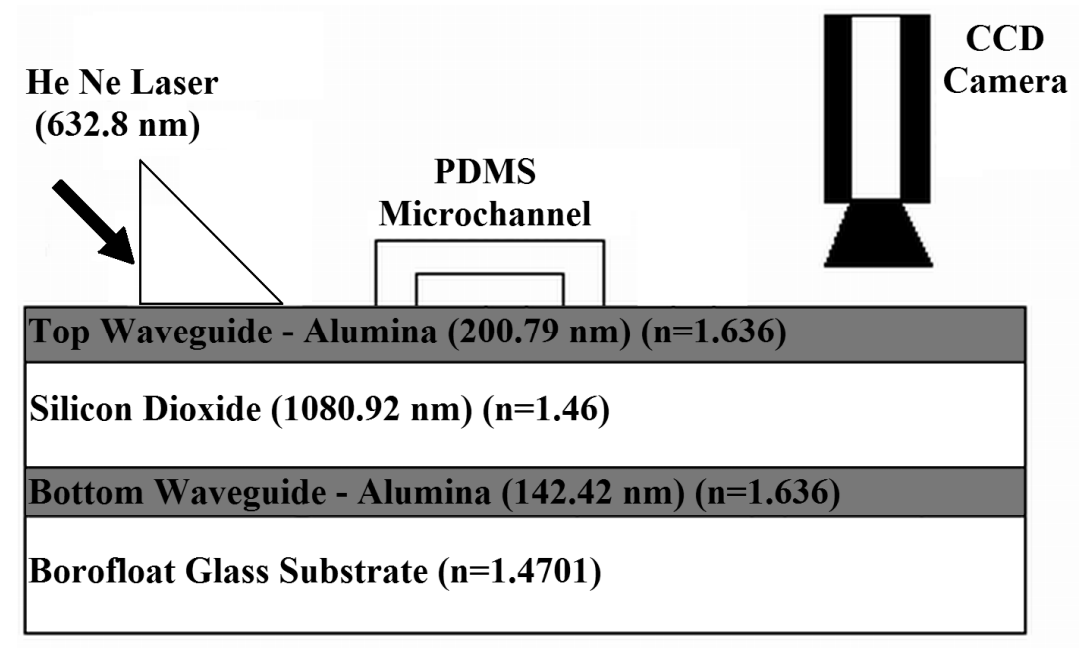

Fig 39 Transducer Structure with a Prism, PDMS microchannel and CCD Camera

The resonant coupled waveguide structure was modeled using Opti-BPM (as shown in section 3-1-2 and 3-1-3) to define the resonant mode profile and to characterize the changes of superstrate refractive index to the resonance condition of the coupled waveguide modes. Two vertically stacked nano-scale aluminum oxide optical waveguides separated by 
a lower refractive index silicon dioxide layer are deposited on a borofloat glass substrate using ion beam assisted deposition (IBAD) technology [76, 77, 78]. Following growth, the structure was annealed at $600^{\circ} \mathrm{C}$ for 10 minutes in an air ambient to reduce optical propagation losses within the thin films. The fabricated structure is shown in figure 39.

A glass top PDMS (Poly-Di-Methyl-Siloxane) channel was constructed on the waveguide stack's surface using the photolithography process as shown in Figure 40. The alumina waveguide structure was first cleaned using a sequence of toluene-acetone-hexanetoluene-acetone sonicator baths for 5-10 minutes each. The waveguides were then dried in the oven at $100^{\circ} \mathrm{C}$ for 20 minutes. Hexa-Methyl-Di-Silazane (HMDS) was spin coated on the substrate at $4000 \mathrm{rpm}$ for $30 \mathrm{sec}$. followed by a soft bake at $150^{\circ} \mathrm{C}$ to improve the adhesion of the photoresist to the substrate. Depending on the desired height of the channel, three to five coats of AZ 4400 positive photoresist was then spin coated each at $300 \mathrm{rpm}$ for $5 \mathrm{sec}$. and $800 \mathrm{rpm}$ for $35 \mathrm{sec}$. Between every coat the wafer was soft baked at $90^{\circ} \mathrm{C}$ for 45 sec with a final bake for 4 minutes at $110^{\circ} \mathrm{C}$. The channel pattern was subsequently exposed and developed to form a PDMS micro-mold. A PDMS mixture (Dow Corning Sylgard 184) was made using a 10:1 ratio of pre-polymer to curing agent and was poured over the channel pattern. The extra PDMS over the photoresist was removed using a sharp blade and the substrate was then baked for 2 minutes at $115^{\circ} \mathrm{C}$ to partially cure the PDMS. The remaining photoresist on the substrate was then removed carefully using acetone. The resulting PDMS walled channel was cured for 2 hours at $150^{\circ} \mathrm{C}$. A glass top with inlet and outlet holes drilled in it was sealed over the PDMS channel using oxygen plasma bonding and capillary tubes were attached to the holes. 


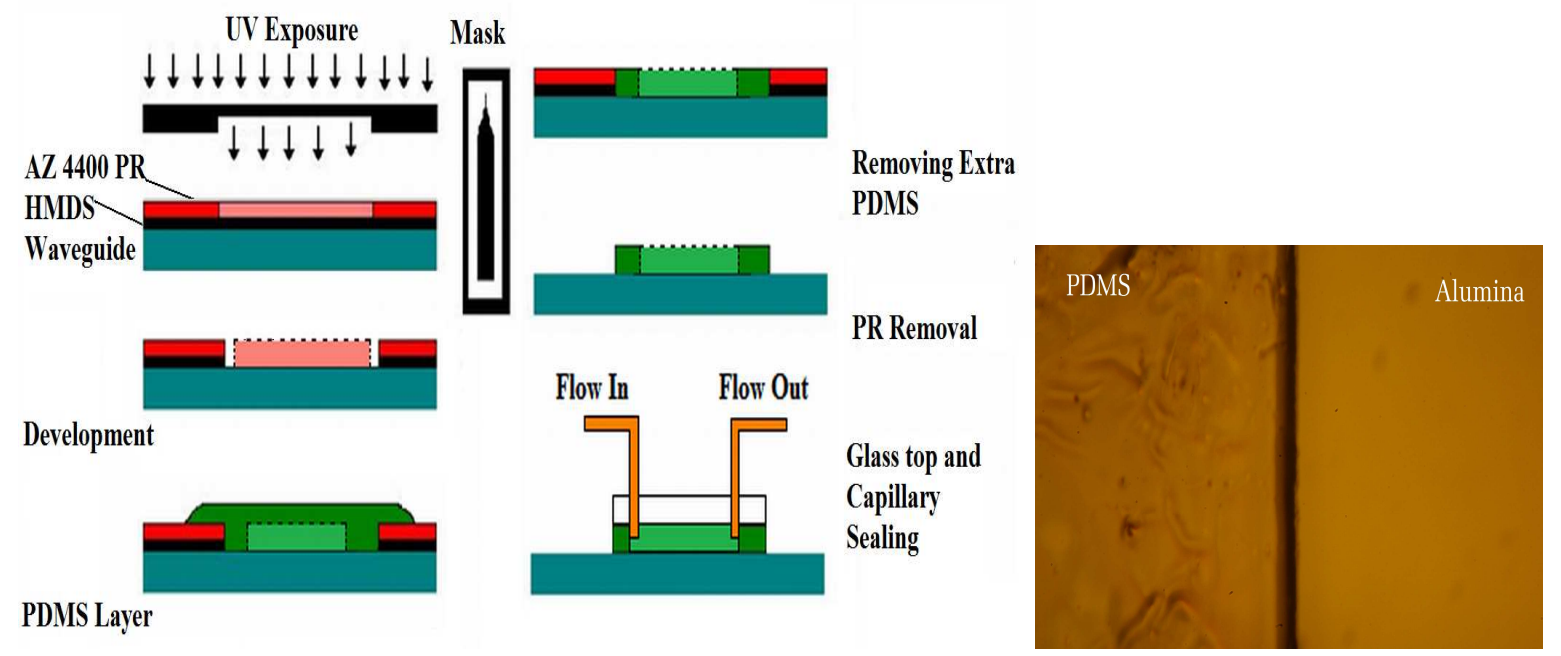

Fig 40 PDMS microfluidic channel fabrication steps and channel sidewall profile

The optical response of the SPARROW transducer was measured as a function of sucrose solution concentration in the microfluidic channel in order to assess sensitivity to uniform aqueous analyte loading of the volume occupied by the evanescent field near the sensor surface. The device under test was mounted in the Metricon 2010 prism coupler and the capillary tubes from the PDMS channel were connected to a Harvard Apparatus 11 plus syringe pump which supplied the desired flow of analyte to the transducer surface. Polarized $632.8 \mathrm{~nm}$ wavelength laser light from a Melles Griot 25-STP-912 stabilized HeNe laser was coupled into the single TE mode supported by the bottom waveguide. The waveguides were designed based on individual film growth thickness and index results such that the mode propagates in the lower waveguide uncoupled to the upper guide in regions of air or PDMS superstrate. Under ideal conditions with waveguide parameters as shown in Figure 39, the waveguide pair is calculated to couple with matched propagation constants when a superstrate of refractive index equal to that of DI water $(n=1.333)$ is present, with waveguide coupling length of $101.34 \mu \mathrm{m}$. The PDMS channel width of $1200 \mu \mathrm{m}$ is chosen to achieve an even multiple of periodic power exchanges. Ideally, under this condition, optical power is fully transferred to the bottom guide after transit of the channel width, when the waveguides again become uncoupled. Increasing the refractive index of the aqueous superstrate above that of water (1.333) changes the propagation constant of the top waveguide, detuning the resonant coupling, resulting in a power reduction measured in the 
bottom waveguide. Also, analyte adsorption can cause scattering loss which reduces the output power. The remaining power in the upper waveguide is scattered out of the guide at the PDMS air interfaces of the channel, as indicated in Figure 41 (a), as well as the additional SU-8 interface applied by hand just after the microchannel. A single waveguide sample was used to experimentally verify that these interfaces reduce power in the top waveguide below the measurement limit of the camera (Figure 41 (b)). Power in the lower guide continues propagating to a region where the waveguide is imaged using the QImaging Retiga 2000R CCD Camera. The pixel values in a fixed region are processed and summed using MATLAB to arrive at a value for the scattered power that is proportional to the power in the waveguide.

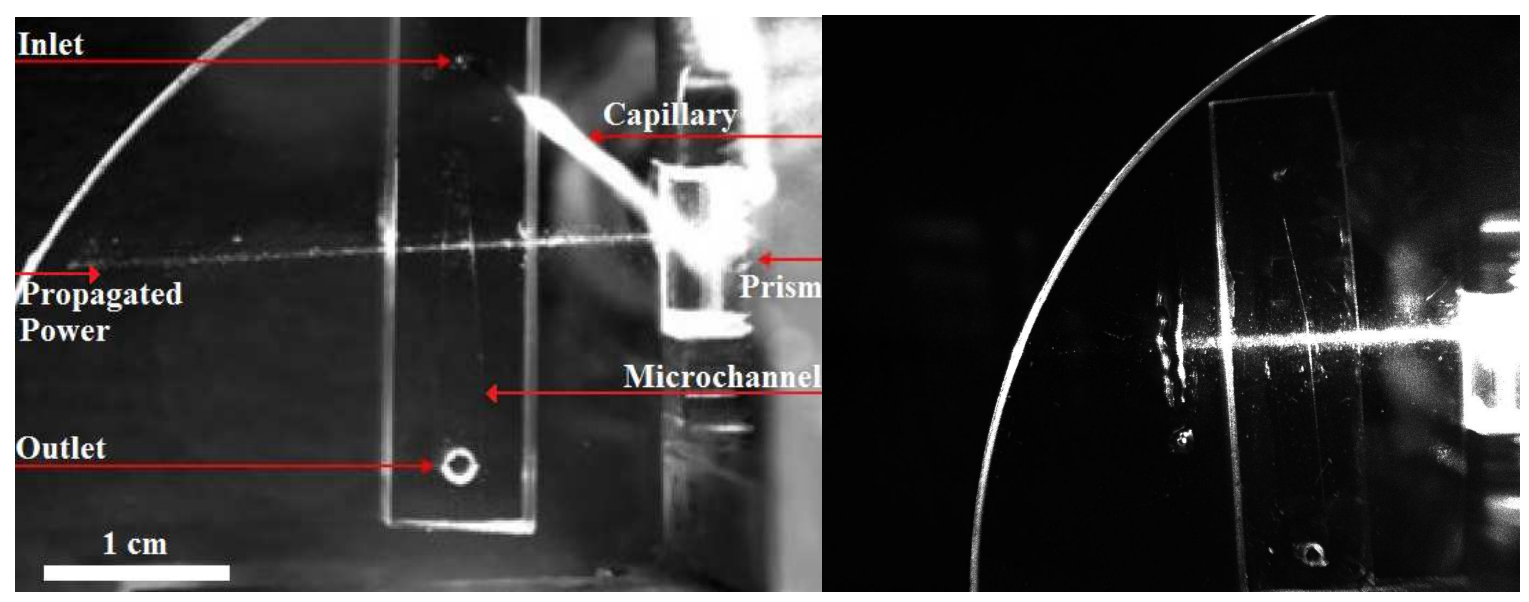

Fig 41 (a) Sample flow setup with power scattered from the top waveguide at PDMSair interface and propagated power in the bottom waveguide and (b) Power drop in a single waveguide with application of SU-8 layer

The transducer optical response was measured by introducing flow of sucrose/DI water solutions of varying concentrations over the device surface using a syringe pump at a flow rate of $2 \mu \mathrm{l} / \mathrm{min}$. Aqueous solutions of concentrations from $0.1 \%$ to $0.5 \%$ sucrose (w/v) were prepared and introduced through the PDMS channel and over the device surface beginning with lowest concentration and progressing to the highest. Prior to the introduction of each concentration, a flow of 1 Molar $\mathrm{NaCl}$ solution followed by DI water was used to clean the microchannel and device surface (as the polar $\mathrm{Na}^{+} \mathrm{Cl}^{-}$desorbs sucrose molecules from the top alumina waveguide surface). Figure 42 shows a plot of the experimentally 
determined change in lower waveguide optical power as a function of sucrose solution loading. For Flow 1, results compare favorably with the simulated result from OptiBPM.

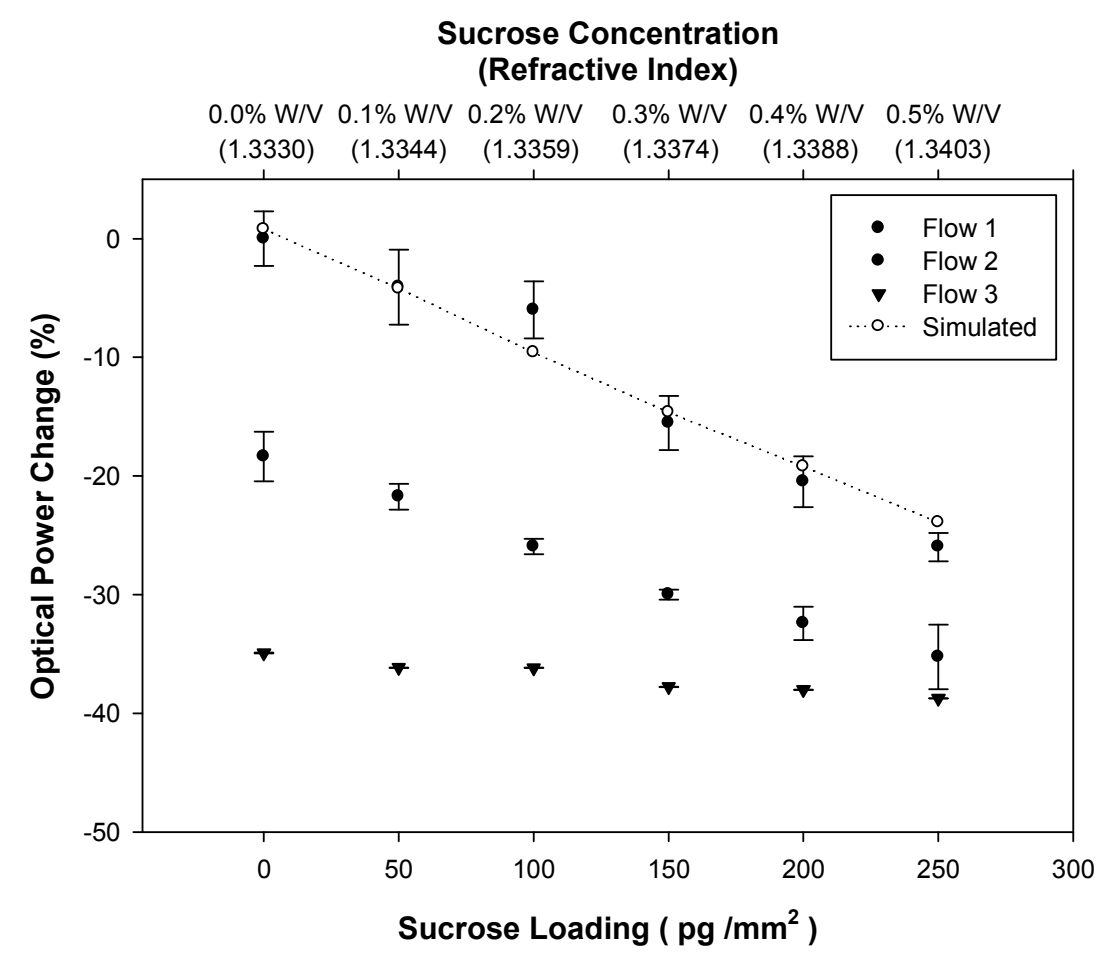

Fig 42 Sucrose Solution Loading vs. Optical Power Change for Three Consecutive Flows and OptiBPM Simulated Transducer Response.

Precise tuning was not achieved due to final film index and interface location uncertainty arising from film stack growth. From this experimental data the minimum detectable surface loading was estimated based on the amount of sucrose in the interaction volume of the top waveguide evanescent field in the channel. The calculated surface loading is shown in Figure 42 given a $0.5 \mathrm{~mm}$ laser beam FWHM, $1200 \mu \mathrm{m}$ channel width and evanescent wave penetration depth of $50 \mathrm{~nm}$. A $50 \mathrm{pg} / \mathrm{mm}^{2}$ loading is seen to result in a $4.084 \%$ optical power drop. Assuming a linear fit to the flow 1 data over this range of concentrations, the minimum detectable percent change in optical power is given by the uncertainty in the slope, or $2.04 \%$. Assuming uniform distribution of sucrose in the interaction volume, the effective limit of detection (LOD) for the waveguide stack is $20 \mathrm{pg}$ $/ \mathrm{mm}^{2}$. This corresponds to an estimated sensitivity with respect to changes in bulk refractive index $\mathrm{S}_{\text {bulk }}$ and effective refractive index $\mathrm{S}_{\text {eff }}$ of $5.6 \times 10^{-4}$ [RIU] (Refractive Index Units) and 
$2.4 \times 10^{-5}$ [RIU] respectively [79]. Successive flow cycles resulted in a continual reduction in the sensitivity of the device despite the cleaning protocol employed. This sensitivity reduction with successive flows is believed to be due to sucrose molecular adsorption on the alumina surface. Water molecules in the aqueous sucrose solution are known to form a stable hydroxide layer and adsorbs in an ordered fashion [80]. The sucrose molecule adsorption on alumina can be described as a metal-saccharide interaction similar to organometallic compounds, and occurs due to the electrostatic forces between the negatively charged ends on disaccharide molecules and positively charged alumina metal groups [81]. The dispersion of sucrose molecules on alumina surfaces and formation of crystalline phase at higher loading is also reported in the literature [82]. Another factor that may have affected the device sensitivity was thinning of the top alumina waveguide surface by the $\mathrm{NaCl}$ solution and the formation of sodium aluminate $\left(\mathrm{Al}_{2} \mathrm{O}_{3}+2 \mathrm{NaCl}+\mathrm{H}_{2} \mathrm{O} \rightarrow\right.$ $2 \mathrm{NaAlO} 2+2 \mathrm{HCl}$ ), potentially causing a reduction in waveguide coupling and sensitivity and reduction in the slope with repeated channel cleanings and sucrose solution flows.

Polyethylene glycol (PEG) of suitable molecular weight is one candidate surface layer that can be applied to the surface to reduce such nonspecific binding in a sensor device implementation while providing a surface for probe and target attachment within the evanescent field penetration depth. The sensitivity of the device to film layer thickness, film refractive index and PDMS channel width variation was calculated. As expected, the device is most sensitive to top waveguide variation with the measurement uncertainty of the present setup corresponding to a change in film thickness of $\pm 0.1 \mathrm{~nm}$, or a film refractive index change of $\pm 7 \times 10^{-5}$ [RIU]. The corresponding sensitivity to variation of the oxide layer between guides is $\pm 47 \mathrm{~nm}$ in thickness and $\pm 1 \times 10^{-3}$ [RIU] in refractive index. The corresponding sensitivity to channel width is $\pm 1 \mu \mathrm{m}$ [83].

\section{3-2 Microsphere Surface Coverage Detection}

As seen in the previous section, a uniform surface loading of SPARROW sensor was characterized using various concentrations of aqueous sucrose solution. The next step 
in the study was to analyze the effect of non-uniform (discrete) loading of polystyrene microspheres on the optical output of a planar waveguide. Surface coverage of nonfunctional attachment of polystyrene microspheres on a planar alumina waveguide was simulated and experimentally characterized.

\section{3-2-1 Microsphere-Waveguide Coupled System Simulations}

The microsphere-waveguide coupled system was simulated using the Opti-FDTD tool and the optical modes of a microsphere resonator were studied. The polystyrene microspheres $(\mathrm{n}=1.5)$, coupled optically from a planar alumina waveguide $(\mathrm{n}=1.636)$ exhibit the presence of Whispering Gallery Modes (WGMs). A $5 \mu \mathrm{m}$ radius microsphere was kept at a distance of $20 \mathrm{~nm}$ from a $0.5 \mu \mathrm{m}$ thick alumina waveguide and a Gaussian Modulated Continuous plane wave (GMCW) in space-time and having a center wavelength of 632.8 $\mathrm{nm}$ and full-width-half-maximum (FWHM) of $\sim 50 \mathrm{~nm}$ was used as the input source. The propagation constants were calculated and matched for coupling to take place and appropriate radius and wavelengths were used for WGM generation. The resonance equation was satisfied and the whispering gallery modes were observed. Also an optical power drop of $1.804 \times 10^{-19}$ Watts was observed at the end of the waveguide which was consumed and scattered by the microresonator.
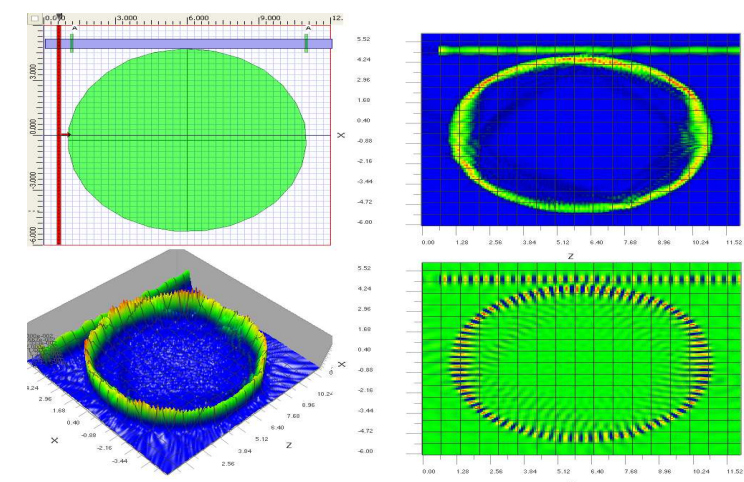

Fig 43 WGMs in an Optically Coupled Microsphere-Waveguide System 
The figure 43 above shows the input plane wave coupled to the waveguide and whispering gallery mode generation in a polystyrene microsphere when the resonance condition is satisfied. Now, changes made to the resonance wavelength will deform the WGM generation as only certain wavelengths can undergo constructive interference for a particular cavity radius. For the same system when the wavelength of $624.39 \mathrm{~nm}$ was used it created a distorted WGM as shown in figure 44 below.
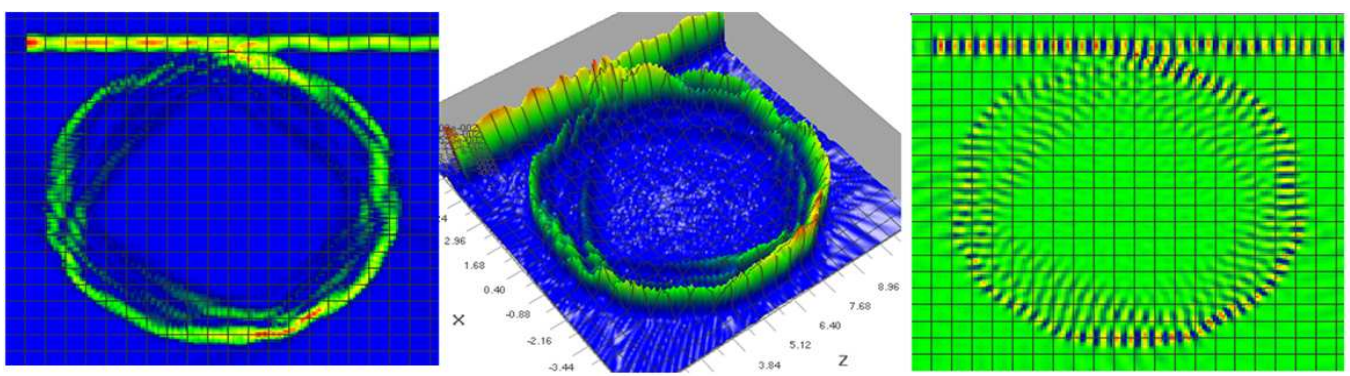

Fig 44 Distorted WGMs at an Off Resonance Wavelength

As the radius of microsphere is increased (at a constant waveguide-microsphere distance) it consumes more power from the waveguide to couple into the WGM. And as the distance between the waveguide and microsphere is increased (at a constant radius) there is a reduction in coupled power as shown in the plots.

Power Loss vs. Microsphere Radius

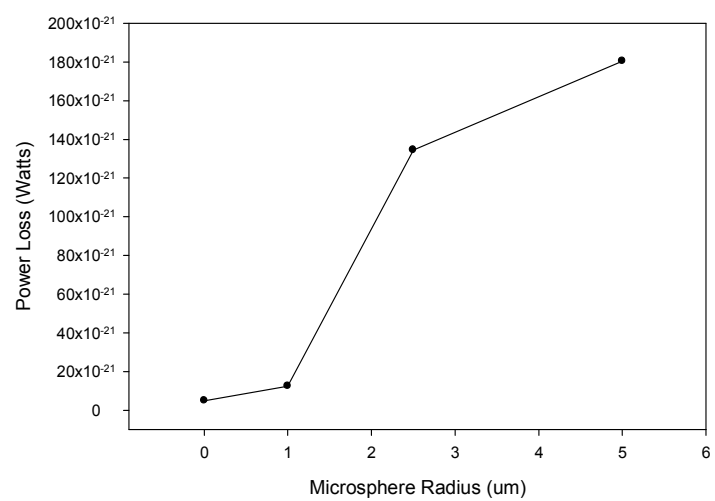

Coupled Power vs. Microsphere Distance

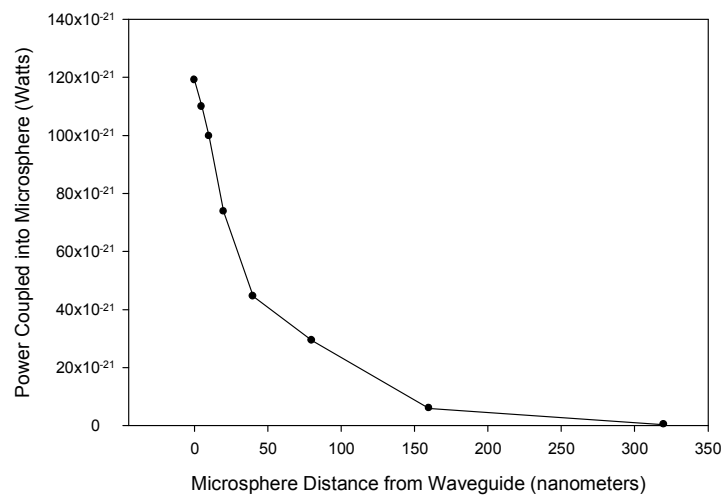

Fig 45 Optical Power Loss in WG vs. Microsphere Radius (Left) and Coupled Power in the Microsphere vs. distance from WG (Right) 

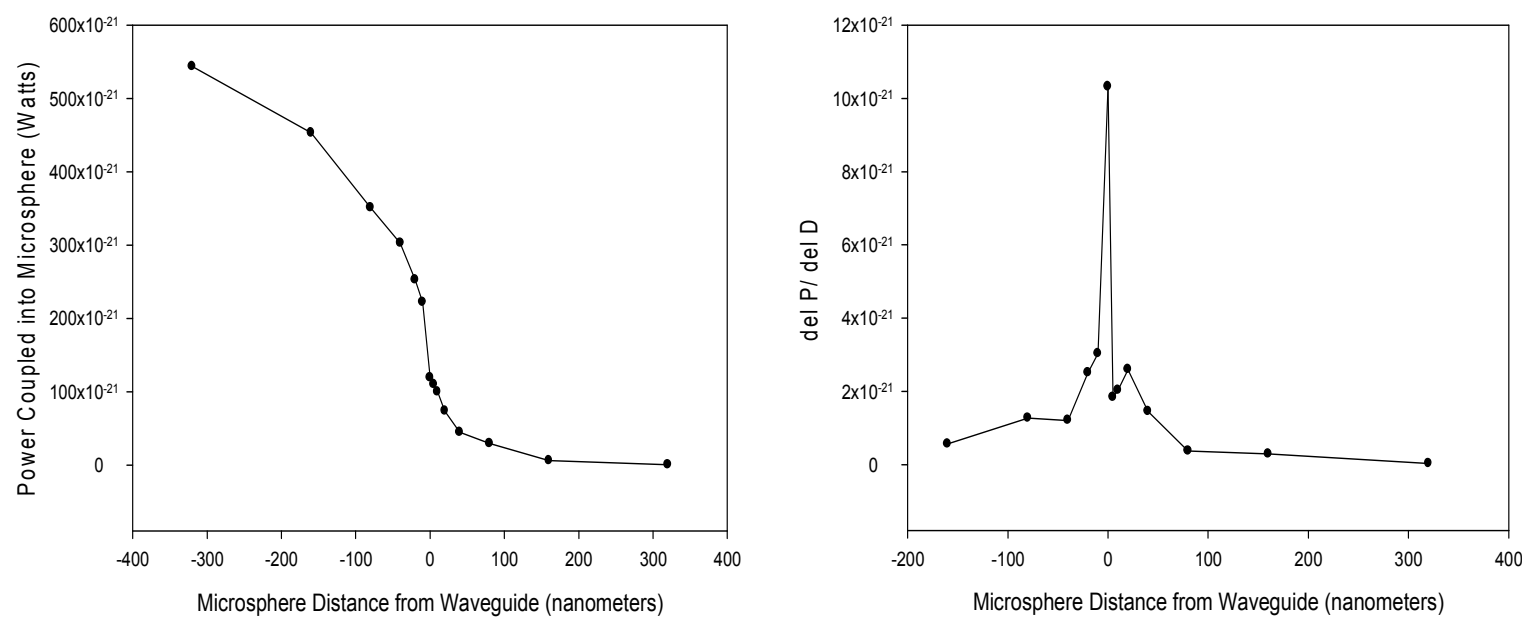

Output Power vs. Microsphere Distance

Output Power Rate Change vs. Microsphere Distance
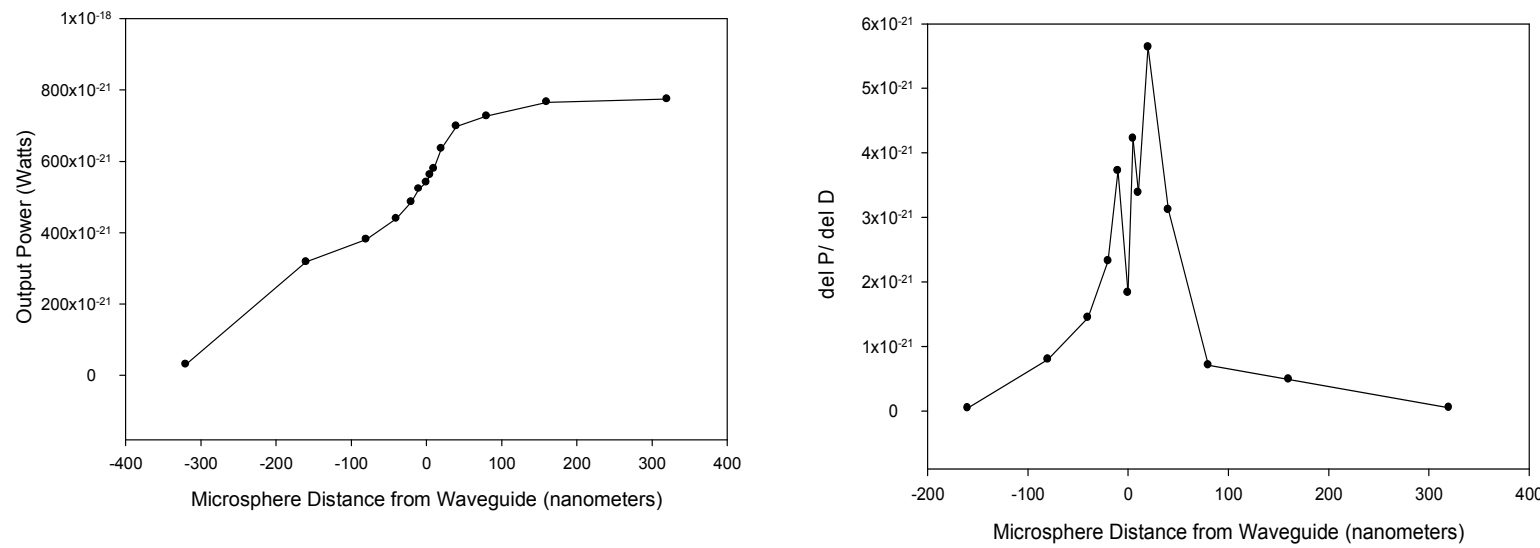

Fig 46 Microsphere Coupled Power (Top) and WG Output Power (Bottom) vs. Coupling Distance and Differential plots (Right)

The microsphere-waveguide coupling distance was varied by moving the microsphere away or into the waveguide and changes in the optical power coupled in the microsphere and output power at the end of the waveguide was characterized as shown in figure 46. The distances of around $10 \mathrm{~nm}$ inside and outside the waveguide were most sensitive to any power changes in the coupled resonator system. Beyond the distance of 150 $\mathrm{nm}$ almost no power was coupled into the microsphere. For a Gaussian wavelength range as 
the optical input coupled into the waveguide, certain resonance wavelengths show a spectral minimum (dips) at the output transmission spectrum. These are the resonant frequencies which undergo constructive interference in the microsphere resonator and hence shown as a reduction in intensity at the output.

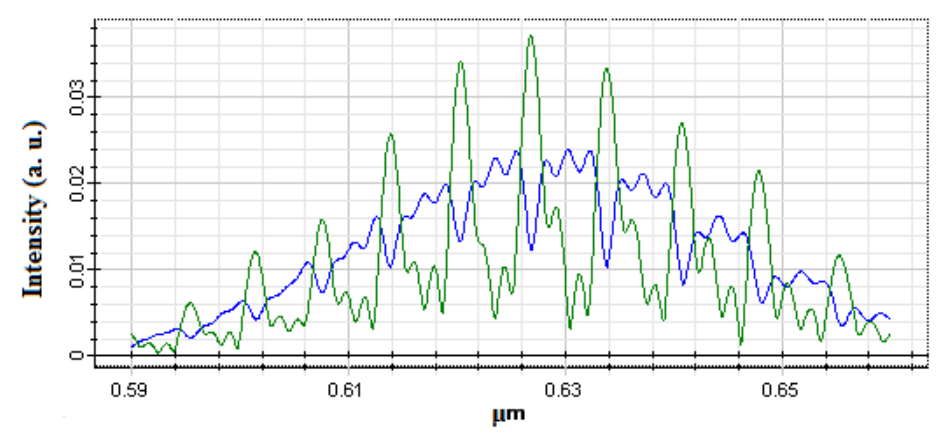

Fig 47 WGMs as Spectral Minima in WG Transmission (Blue) and WGM Peaks inside Microsphere Cavity (Green)

Any changes in the microsphere radius can alter the coupled intensity of whispering gallery modes into the microsphere as shown in figure 48 . A change in $35 \mathrm{~nm}$ of the radius can deform the resonance condition.
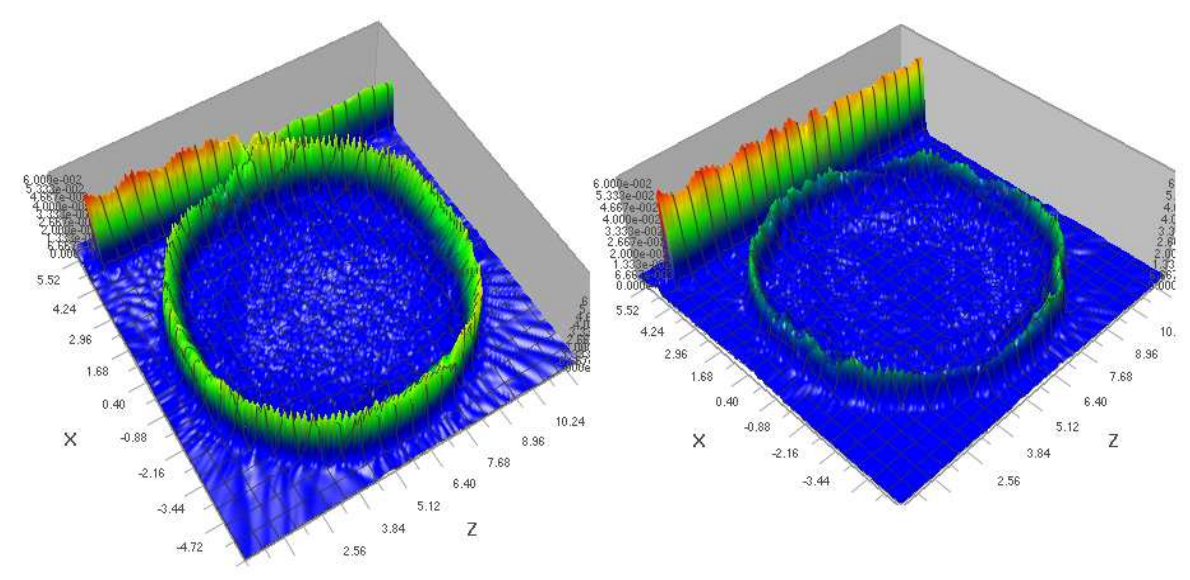

Fig 48 On-Resonance and Off-Resonance when the microsphere radius is increased by $35 \mathrm{~nm}$ 
Also, a shift of $1.194 \mathrm{~nm}$ of resonant spectral peaks is observed when the radius of the microsphere was increased by $35 \mathrm{~nm}$.

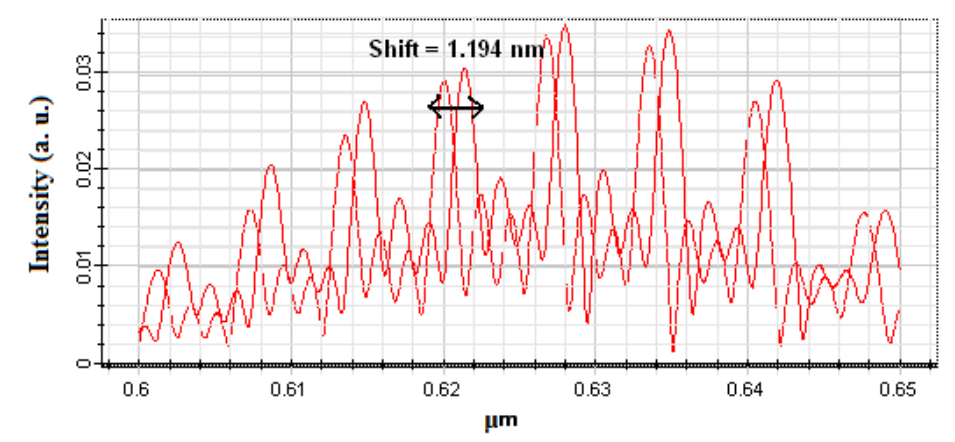

Fig 49 Shift of resonance peaks with the radius change of the microsphere

Hence, simulation study shows that any changes made in the cavity radius or refractive index of the surroundings can alter the on-resonance condition of the whispering gallery modes, and can be used as a sensing mechanism for molecular binding. Besides the presence of WGM, the microspheres exhibit internal coupling losses and external scattering losses which are seen in the next section.

\section{3-2-2 Scattering Loss Analysis Simulations}

Using equations $(2.46,2.53)$ of the theory chapter, the microsphere internal and external optical losses were simulated using MATLAB. The losses were characterized by changes in the refractive index, radius and surface roughness of the microsphere. 


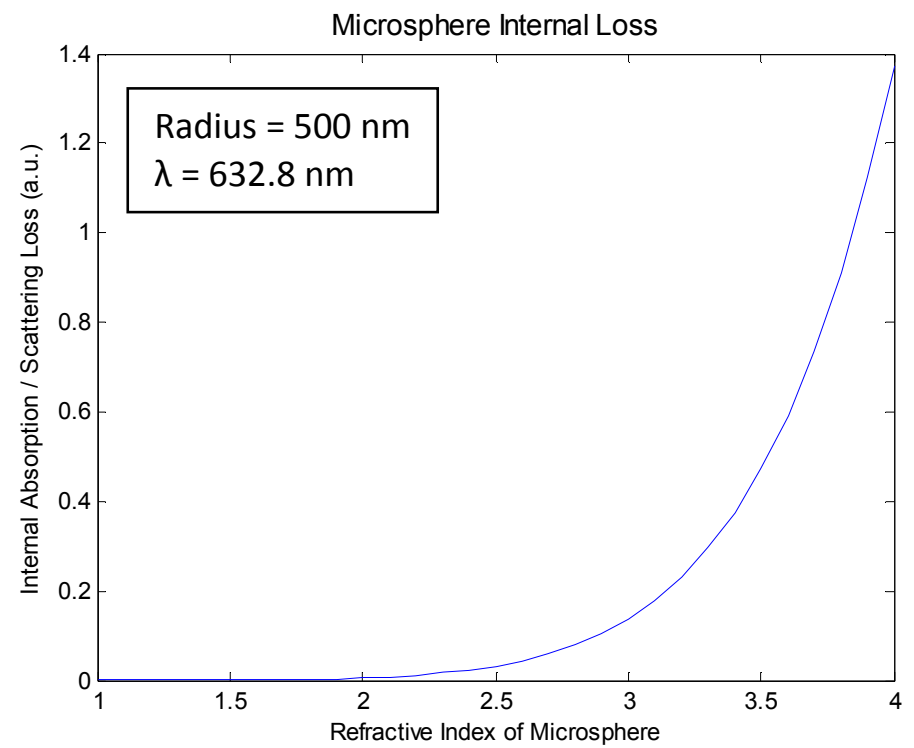

Fig 50 Microsphere Internal Optical Losses vs. Refractive Index

The above shown MATLAB plot was generated for the microsphere internal losses $\left(\boldsymbol{\alpha}_{\text {int })}\right.$ with respect to change of microsphere refractive index at a wavelength of $632.8 \mathrm{~nm}$ for a $1 \mu \mathrm{m}$ diameter microsphere. (The MATLAB program is given in the Appendix).

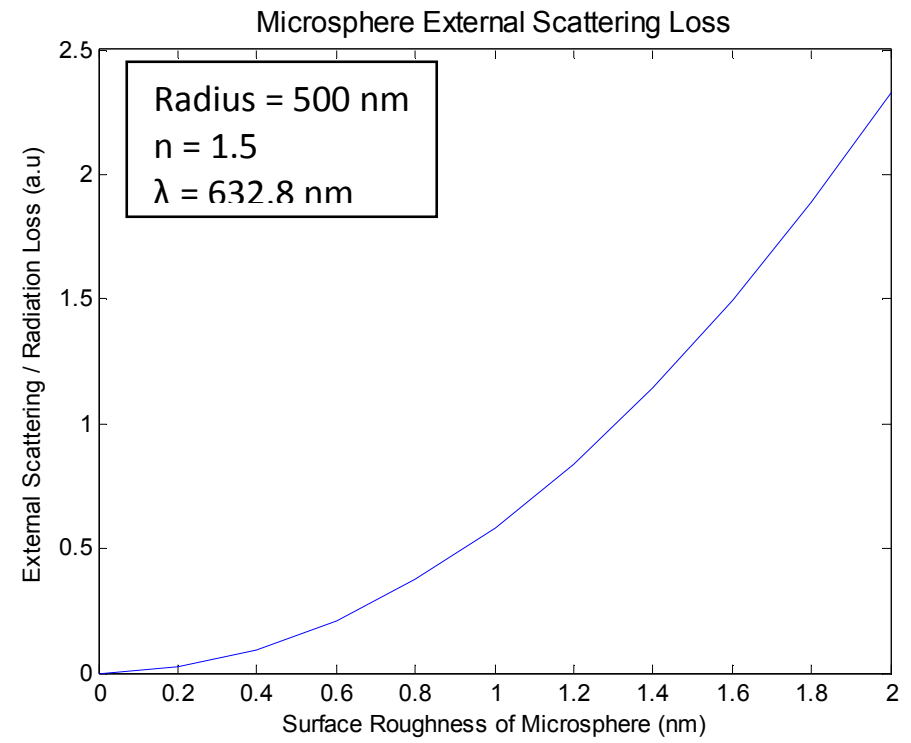

Fig 51 Microsphere External Optical Losses vs. Surface Roughness 
The MATLAB plot above is generated to analyze the external radiation losses $\left(\boldsymbol{\alpha}_{\text {ext) }}\right.$ with respect to surface roughness of the microsphere. By comparing the two plots from the internal and external scattering, we can conclude that the microsphere internal absorption losses were negligible for the polystyrene index $(n=1.5)$ but the external losses were significant at the polystyrene index of 1.5 and vary greatly with the surface roughness changes.

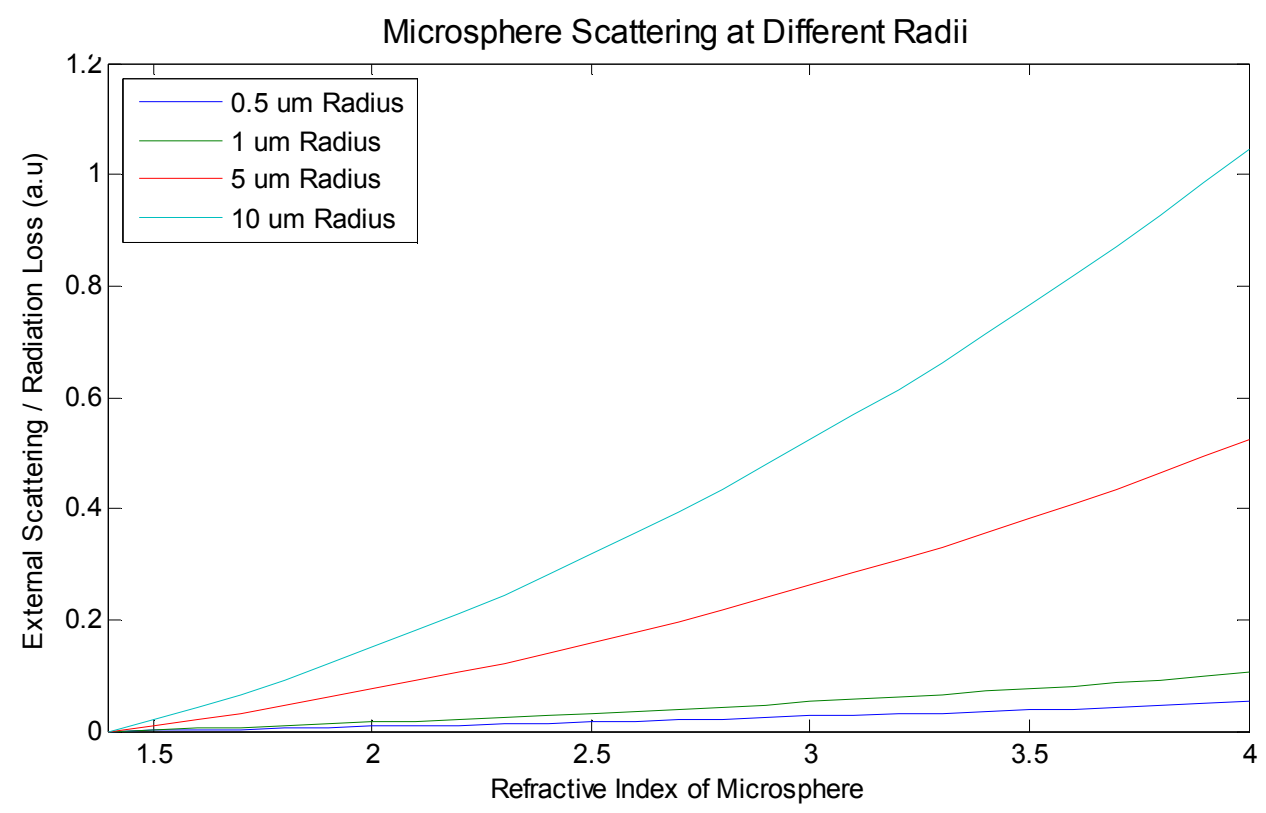

\section{Fig 52 Microsphere External Scattering Losses vs. Refractive Index at Various Radii}

The external scattering losses of microspheres at various radii with respect to refractive index are shown above in figure 52. As the radius is increased the photon path length and surface roughness parameters increase causing a higher scattering loss. 

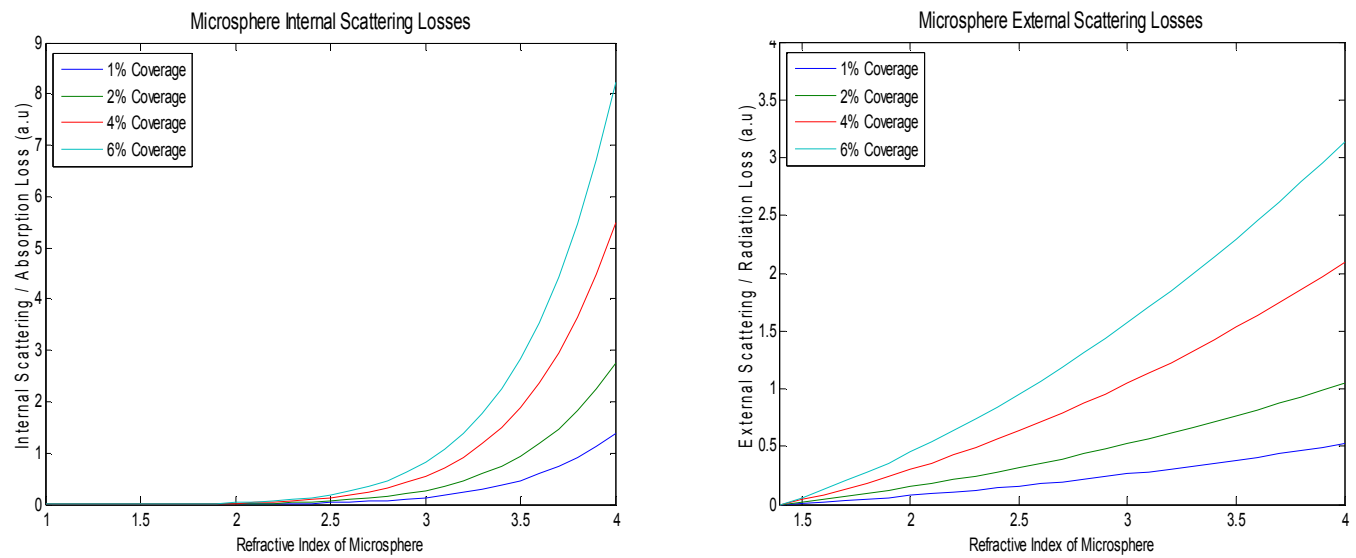

Fig 53 Microsphere Internal and External Losses vs. Refractive Index at Various

\section{Surface Coverages}

The Microsphere internal and external scattering losses corresponding to various surface coverage's are shown in the plots above (figure 53). The surface coverage factor ' $S$ ' was multiplied and plotted as $\mathbf{S} *\left(\boldsymbol{\alpha}_{\text {int }}\right)$ and $\mathbf{S} *\left(\boldsymbol{\alpha}_{\text {ext }}\right)$ with the coverage's varying as $1 \%$, $2 \%, 4 \%$ and $6 \%$.

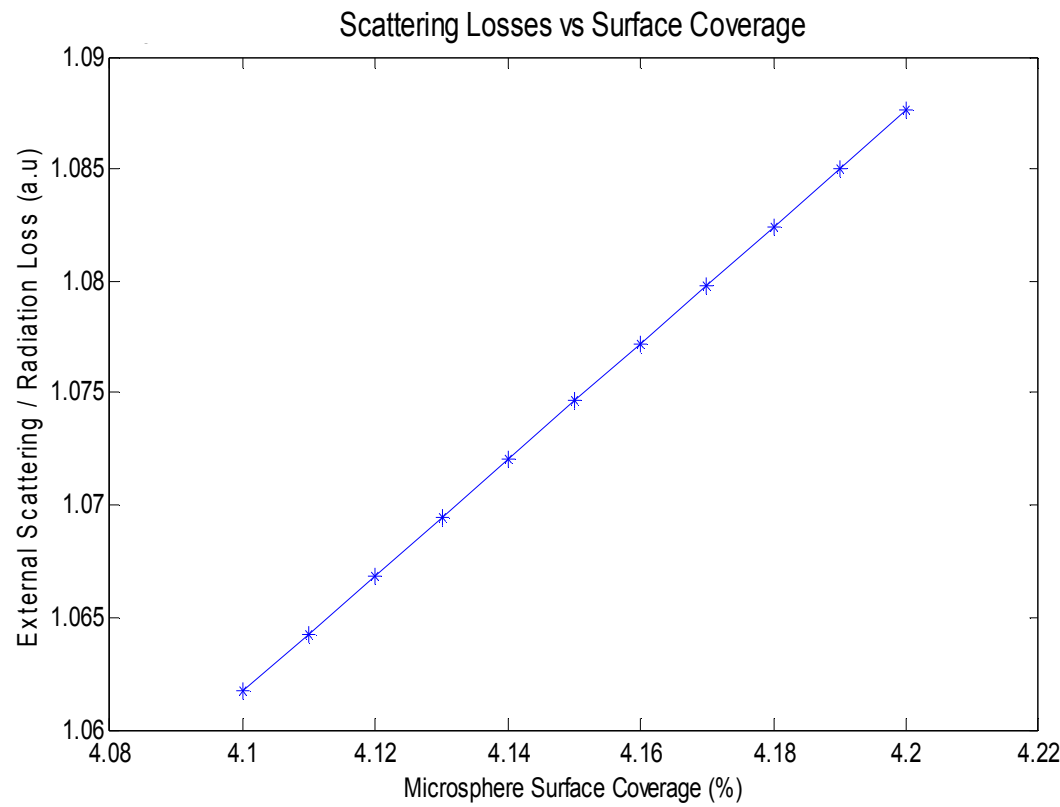

Fig 54 Microsphere External Losses vs. Microsphere Surface Coverages 
Assuming that the losses in the planar waveguide due to surface roughness are constant (As discussed in theory chapter), and neglecting the internal absorption losses into the microspheres at $n=1.5$. The microsphere coupled waveguide's output power change is greatly dependent on the external scattering losses of the microsphere $\mathbf{S} *\left(\boldsymbol{\alpha}_{\mathbf{e x t}}\right)$ and the surface coverage (\%). The MATLAB theoretical plot above (figure 54) shows the increase of external scattering losses by $\sim \mathbf{3 \%}$ due to an increase of surface coverage from $4.1 \%$ to $4.2 \%$. The simulated results can be compared to the experimental characterization for a planar waveguide-microsphere coupled system for study of microsphere surface coverage detection in the next section.

\section{3-2-3 Experimental Results}

The surface coverage of non-functionally attached polystyrene microspheres on a planar alumina waveguide was analyzed using two approaches. First, the microspheres were flown in a PDMS microchannel fabricated on alumina waveguide surface, and optically fluorescently excited using a $488 \mathrm{~nm}$ Yellow-Green Argon-Ion laser. The second approach was to optically excite the waveguide-coupled-microspheres using a $632.8 \mathrm{~nm}$ HeliumNeon laser. The results from two different approaches were compared to investigate the self-assembly of microspheres on alumina waveguide.

\section{3-2-3-1 Fluorescent Experiments}

The surface coverage of non-specific binding of Invitrogen Fluospheres (2\% solids) on a planar alumina waveguide was analyzed using $488 \mathrm{~nm}$ optical excitation of an ArgonIon laser. Carboxylate ions have been reported previously to be chemisorbed on alumina surfaces [82]. Carboxylate-modified fluorescent polystyrene microspheres with $500 \mathrm{~nm}$ radius and negative surface charge were used. The $2 \%(\mathrm{w} / \mathrm{v})$ microsphere solution was made by dilution in a medium solution (50 mM Sodium Phosphate, $50 \mathrm{mM}$ Sodium 
Chloride, $5 \mathrm{mM}$ Sodium Azide, 0.02\% Tween Detergent and DI water). The microchannel was first rinsed with ethanol at $5 \mu \mathrm{l} / \mathrm{min}$. for 10 minutes for cleaning purposes, followed by $2 \%$ microsphere solution flow at $0.5 \mu \mathrm{l} / \mathrm{min}$. for 60 minutes to achieve maximum surface coverage saturation. After the microsphere attachment on the waveguide (in the PDMS microchannel) reaches saturation then a variable flow of medium solution from $15 \mu \mathrm{l} / \mathrm{min}$. to $25 \mu 1 /$ min was flown to analyze the microsphere detachment rates in real time. The microsphere analyte target was characterized for mass transport and attachment/detachment events on the waveguide surface and the surface coverage was calculated. A high quality CCD camera (QImaging Retiga 2000R) with exposure time of $100 \mathrm{~ms}$ focused near the waveguide surface was used to capture an image inside a fixed region of the microchannel to analyze the light scattered by the attached microspheres. A MATLAB code measures the total pixel intensity of the scattered light in the captured image, which measures the surface coverage percentage as shown in Figure 55, 56 and 57.
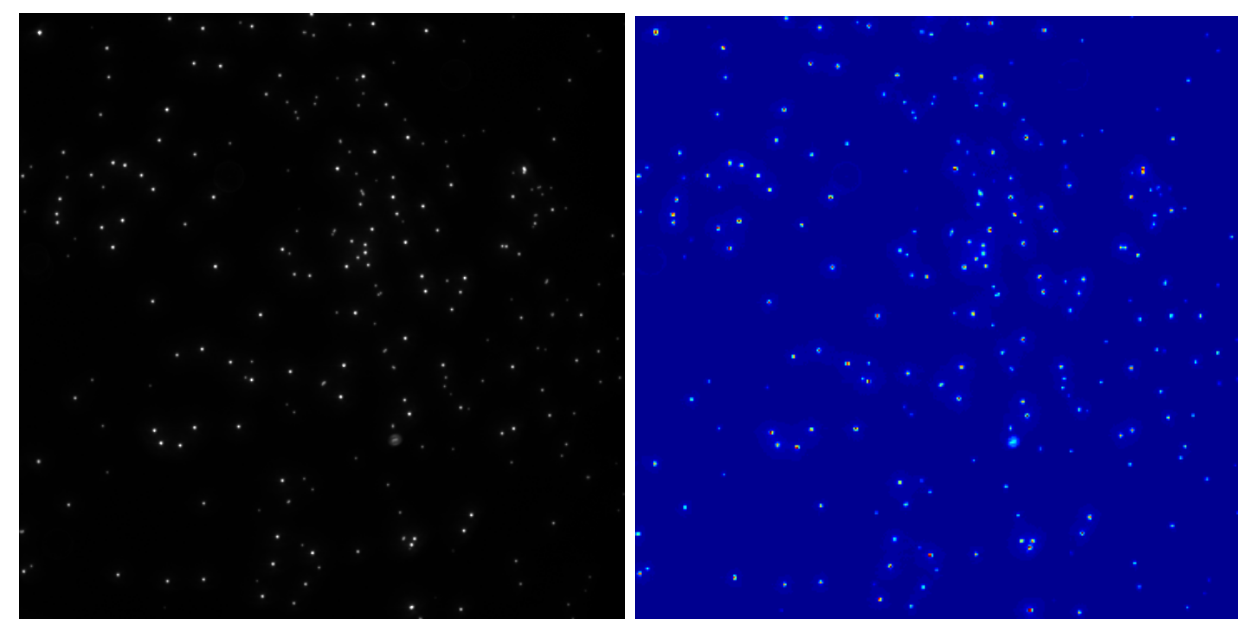

Fig 55 Microsphere Binding on Waveguide Surface (left) and MATLAB Processed Image (right) with Surface Coverage $4.4263 \%$ 

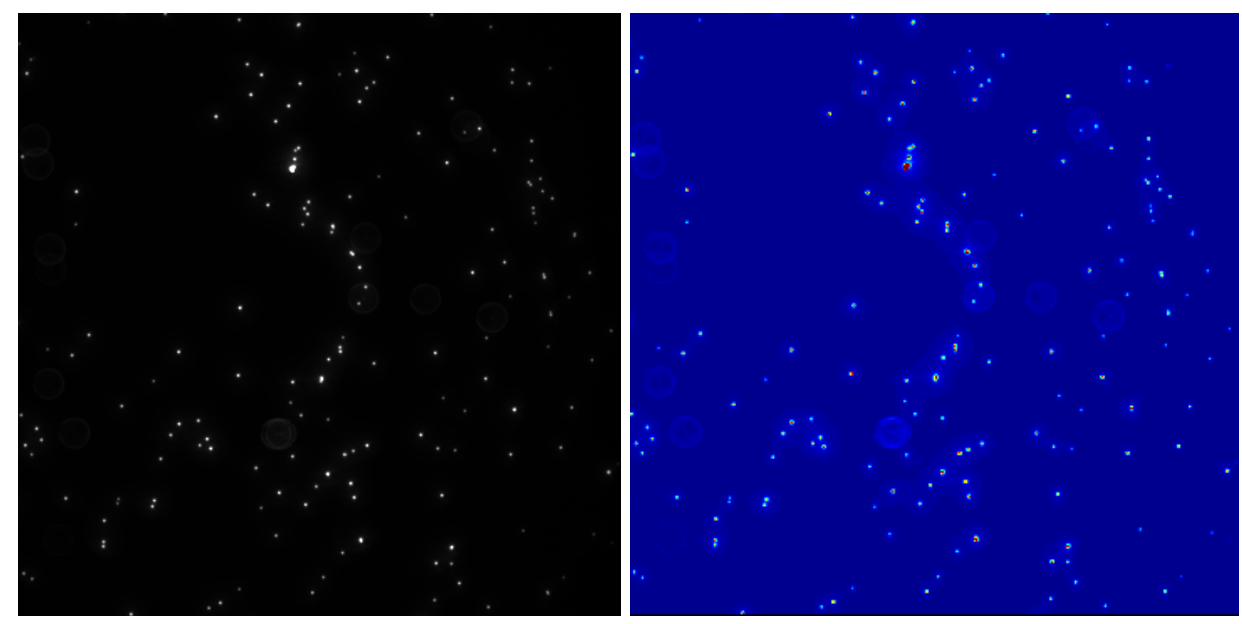

Fig 56 Microsphere Binding on Waveguide Surface (left) and MATLAB Processed Image (right) with Surface Coverage $4.268 \%$
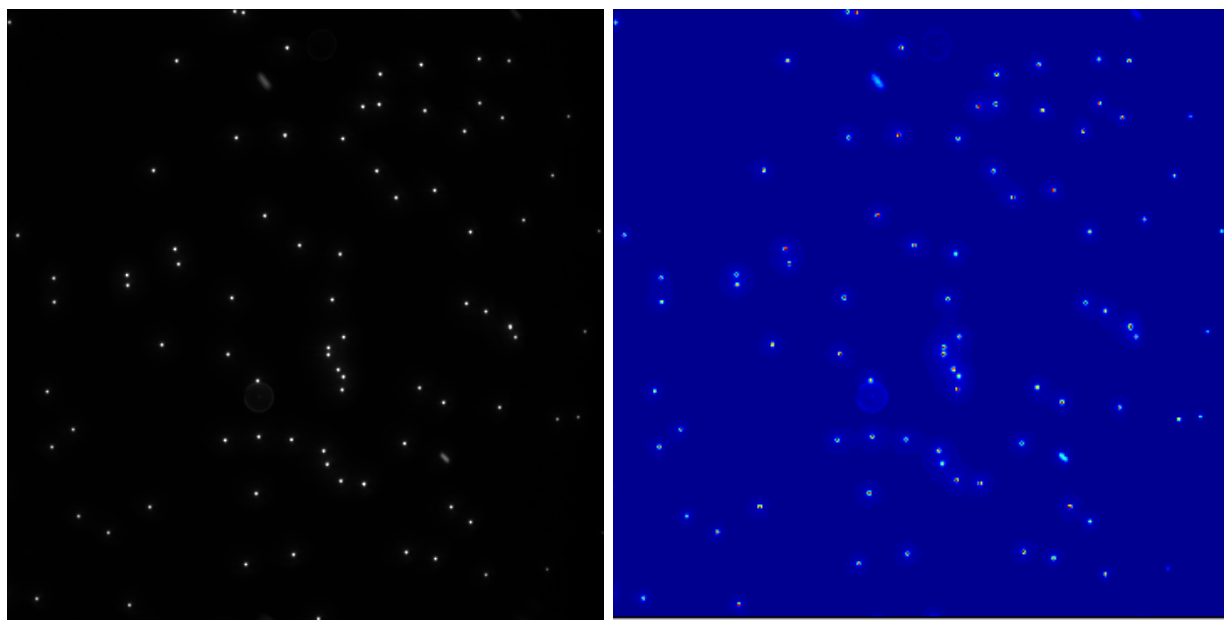

Fig 57 Microsphere Binding on Waveguide Surface (left) and MATLAB Processed Image (right) with Surface Coverage 4.0983 \% 


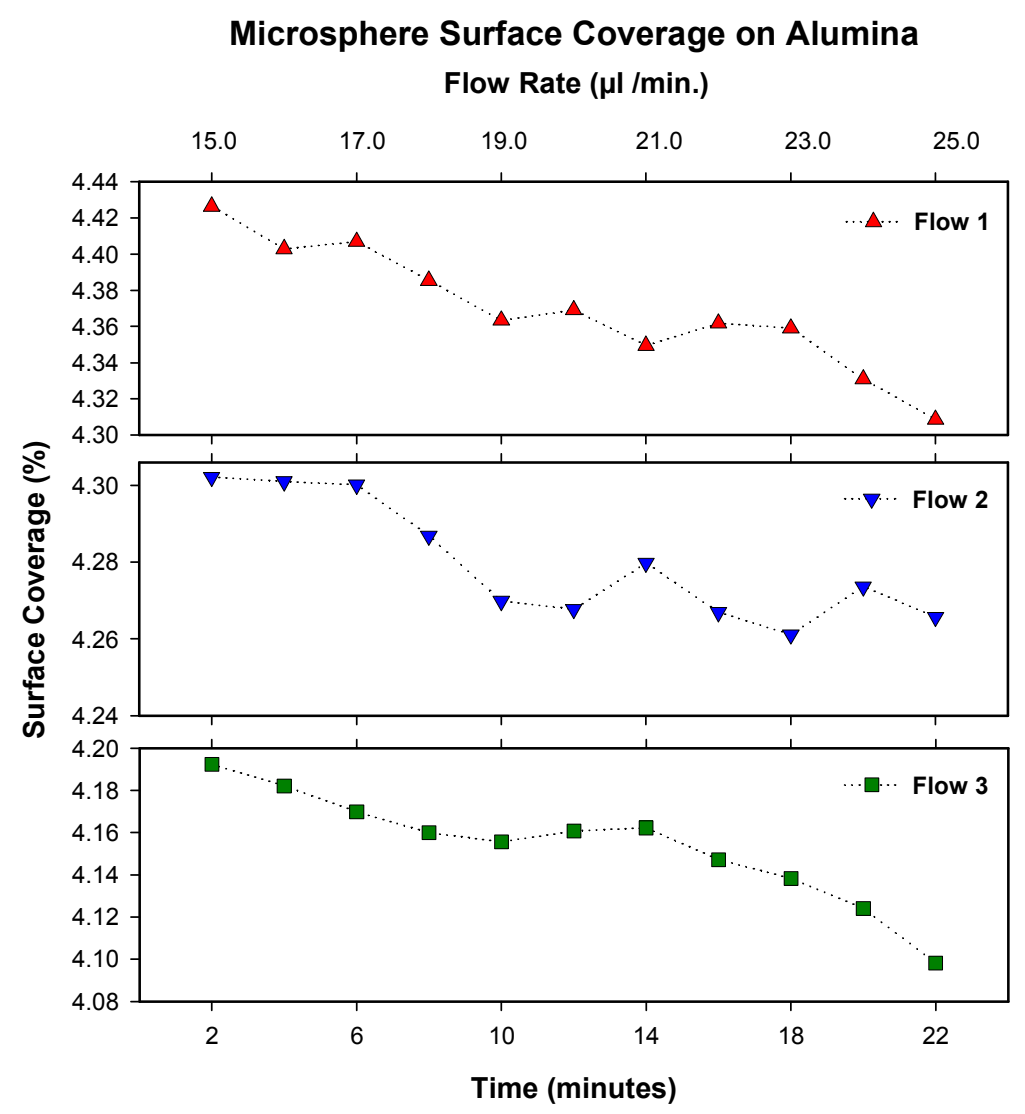

Fig 58 Real Time Measurements for the Microsphere Surface Coverage Binding on Alumina for Repetitive Flows

Consecutive flows of medium solution at different flow rates in time were measured and the salts and detergent in the medium solution were used to weaken the carboxylatemetal oxide bonds for detachment of microspheres. A reduction in surface coverage from $4.426 \%$ to $4.098 \%$ was observed for three flow measurements over 66 minutes with an incremental flow rate of $15 \mu \mathrm{l} / \mathrm{min}$. to $25 \mu \mathrm{l} / \mathrm{min}$. The minimum surface coverage detection limit for microspheres was calculated from the plot to be $8.6 \times 10^{-4} \%$, and the microsphere surface coverage changes in the microchannel with repetitive flows are shown in figure 58. 


\section{3-2-3-2 Prism Coupling Experiments}

The optical sensitivity of a planar alumina waveguide was measured to nonuniformly distributed, discrete adsorption events of carboxylate coated polystyrene microsphere on the waveguide surface and compared to fluorescently excited microsphere surface coverage.

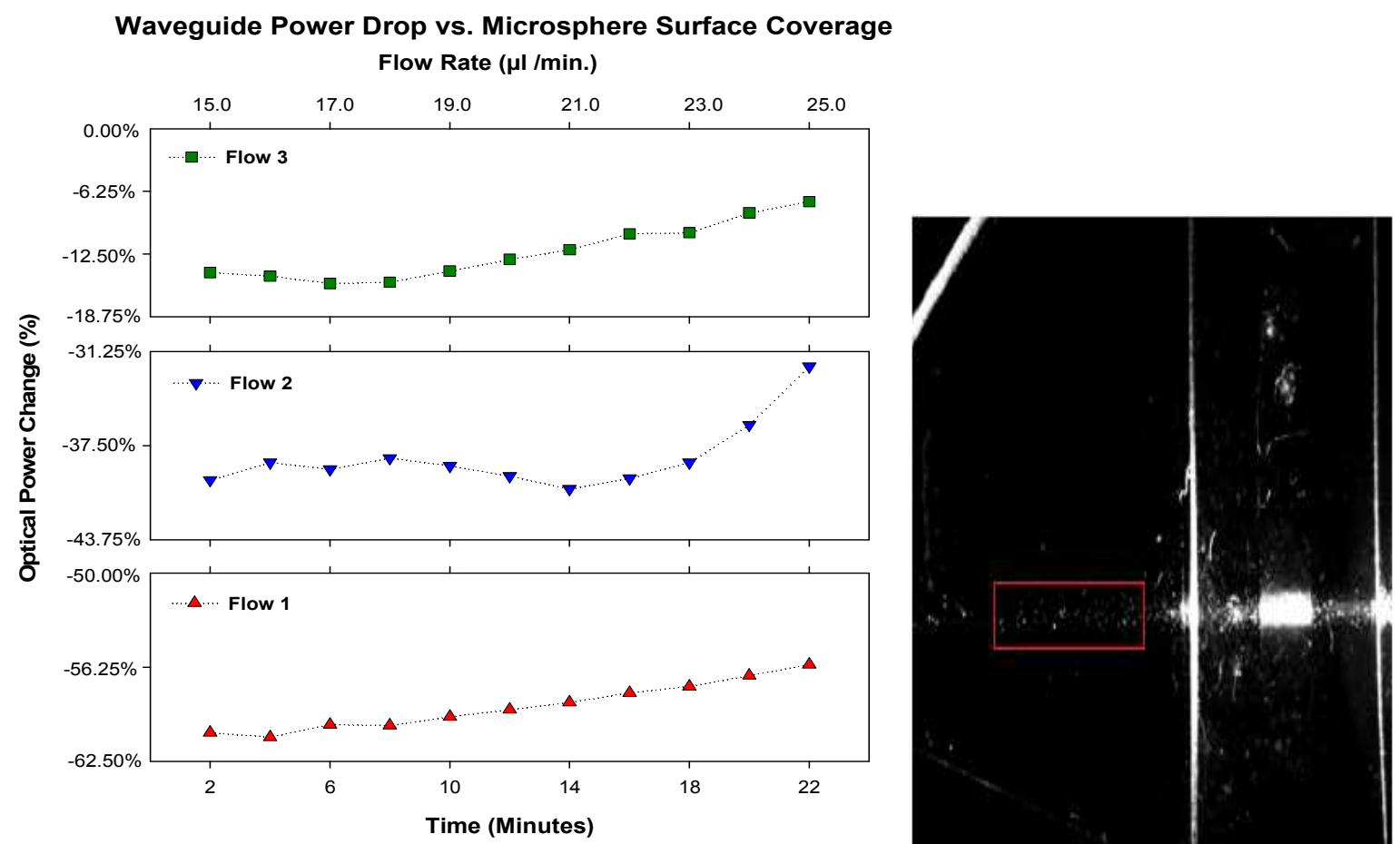

Fig 59 Optical Power Drop in the Alumina Waveguide due to Microsphere Binding (Left) and Microsphere Scattering inside PDMS Microchannel (Right)

The plots in figure 59 shows the percentage drop in optical power in a planar alumina waveguide, showing the maximum power drop of $61.25 \%$ at the maximum microsphere surface coverage of $4.426 \%$ and lowest drop of $7.5 \%$ with surface coverage of $4.098 \%$. The minimum surface coverage detection limit for optical power drop was calculated to be $0.192 \%$. With consecutive flows the microsphere detachment from alumina surface caused a lower power drop at the output. Hence the planar waveguide propagates higher optical intensity at the output when a lower surface coverage of microspheres is 
attached on the surface, which causes a reduction in microsphere scattering losses. Whereas a higher surface coverage results in a higher power drop at the waveguide's output. The discreet binding events in the microchannel were responsible for the changes in effective refractive index and the coupling coefficients of the waveguide-microsphere system. Therefore, comparable optical power changes were measured at the output.
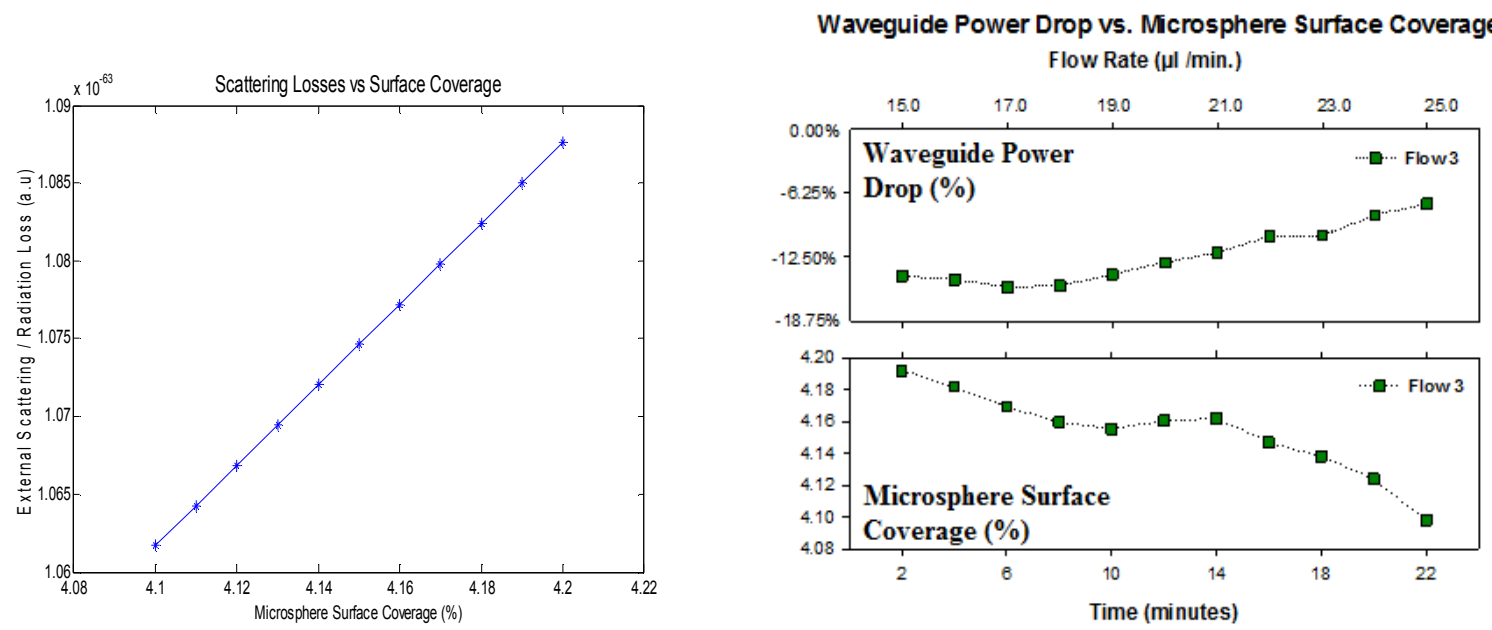

\section{Fig 60 Simulated Microsphere Scattering Losses and Experimental Surface Coverage and Waveguide Power Loss}

The MATLAB theoretical plot above (figure 60) shows an increase of microsphere external scattering losses by $\sim \mathbf{3} \%$ for an increase of surface coverage from $4.1 \%$ to $4.2 \%$. Whereas, the experimental plot on the right side however shows a drop of waveguide power of $\sim 6 \%$ when surface coverage was increased from $4.1 \%$ to $4.2 \%$. The other $3 \%$ power drop in the waveguide is assumed to be due to coupling losses between waveguide and microspheres and internal absorption losses which is a subject of future work.

\section{3-3 Whispering Gallery Modes Detection in GaN Disks}

The experimental plan to obtain Whispering Gallery Modes (WGM) will be reviewed in this section. Gallium Nitride microdisks were chosen as the desired material for 
presence of WGMs for its potential applications in photonics devices and specially LEDs. A passive detection of optical modes was characterized before analyzing the VCMs (Vertical Cavity Modes) in the active LED structures (as explained in section 3-4).

\section{3-3-1 Passive GaN Disk Simulations}

Opti-FDTD modeling software was used to design and analyze the WGMs in GaN Micro-Disks. The electromagnetic fields were characterized both inside and outside the disk cavity. A gaussian modulated plane wave was used to excite the disks externally, and to simulate the passive excitation of disks. Whereas Gaussian modulated spherical point sources were used to excite the disks internally and to investigate the WGMs generated in an active cavity. The Gallium Nitride micro-disks were used as photonic resonant structures for inducing whispering gallery modes for sensing applications. The Gaussian modulated wave-packet function is shown below:

$$
e^{-\frac{1}{2}\left(\frac{t-t_{o}}{W}\right)} \sin (\omega t)
$$

Where, $\boldsymbol{t}_{\boldsymbol{o}}$ is the time offset and $\boldsymbol{W}$ is the half width. 


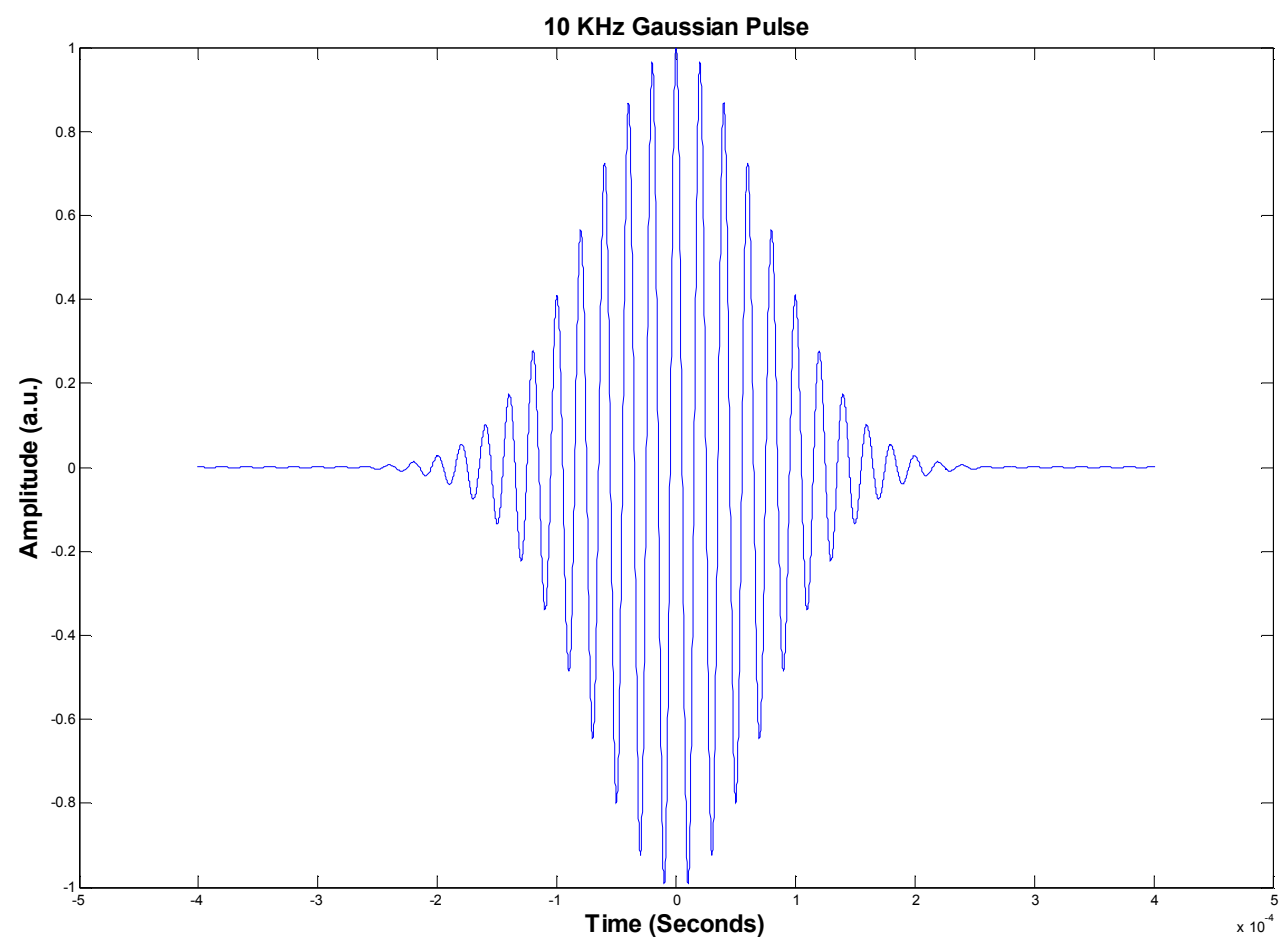

Fig 61 MATLAB Simulated Gaussian Modulated Wave-Packet Pulse

The whispering gallery mode simulations of a gallium nitride disk were similar to the microsphere-waveguide system seen in the previous section. The internally coupled spherical wave optical point sources inside the cavity were modeled, and the cavity modes were analyzed. A single point source positioned at the center of the GaN disk creates a spherical wave, and as all of the wave fronts are normal to the disk boundary, total internal reflection does not take place and no WGMs were observed. 


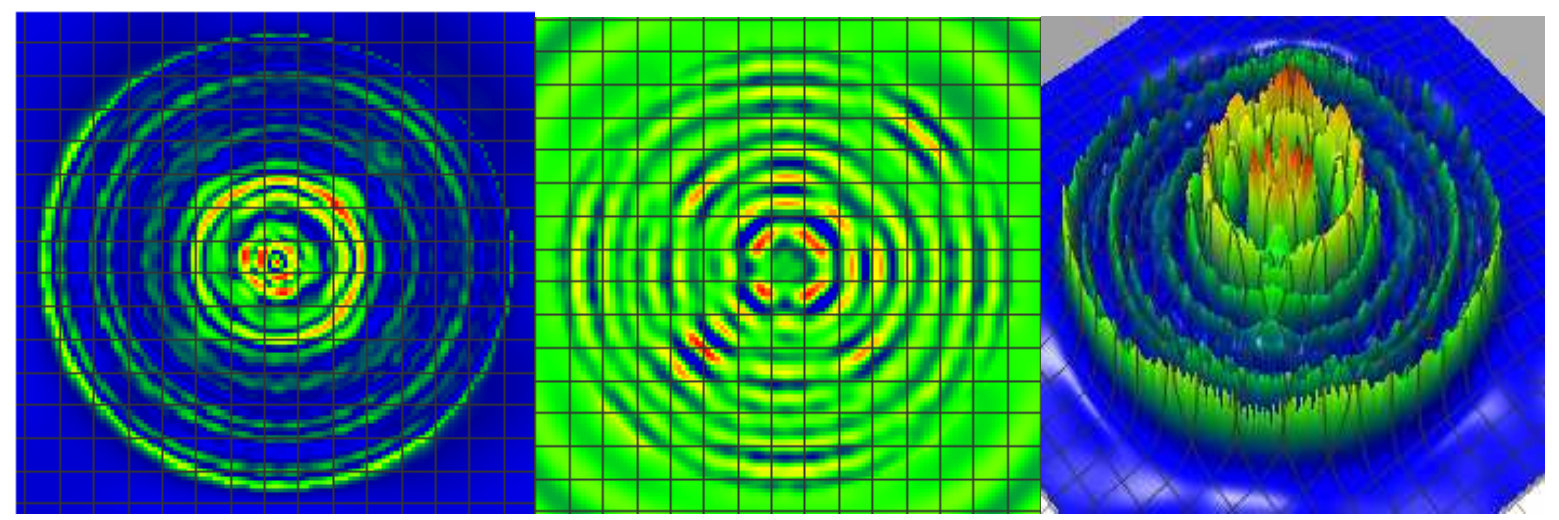

Fig 62 Internally Excited GaN Disk with a Single Point Source at the Center

The GaN disk of radius $1.65 \mu \mathrm{m}$ and thickness $200 \mathrm{~nm}$ was used with 8 centered point sources at a peak wavelength $423.32 \mathrm{~nm}$. The modeled disk is shown in the figure below.

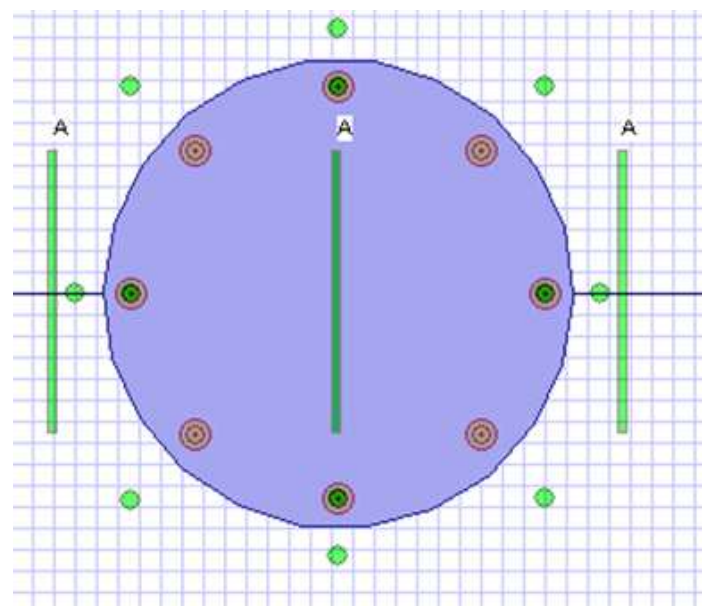

Fig 63 GaN Microdisk with Point Sources in Red and Observation Points and Planes in Green

The simulation shows multiple radial whispering gallery modes generated in the GaN disk and various positions of the point sources can excite different mode profiles in the disk. 

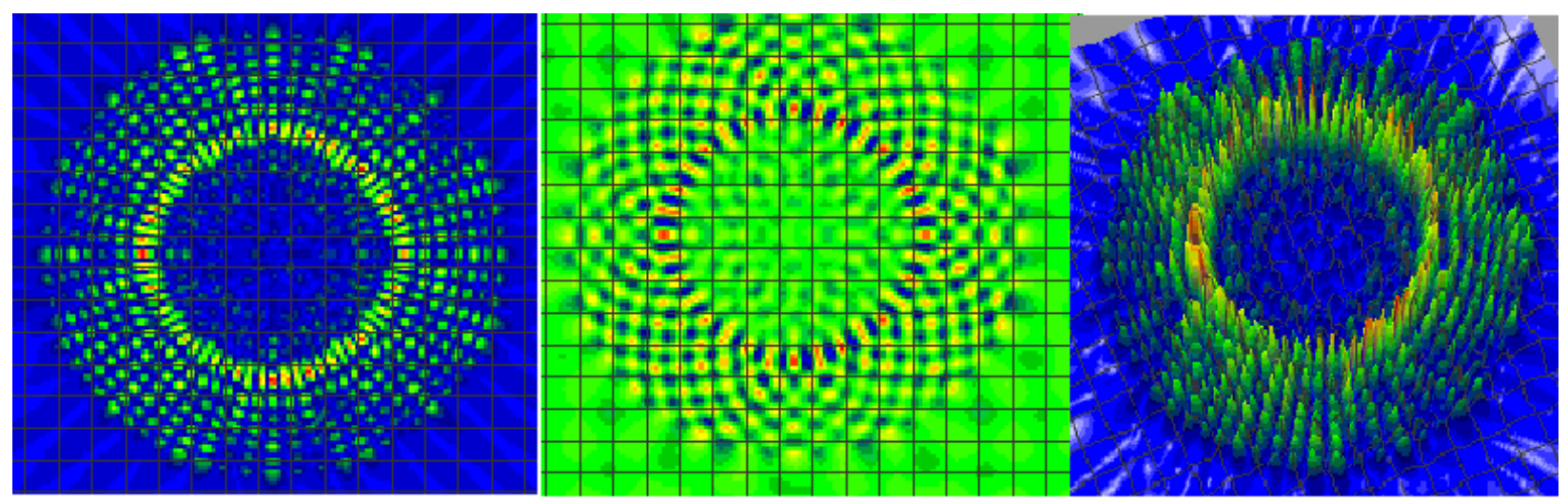

Fig 64 Opti-FDTD Simulations of GaN Disk with Multiple WGMs

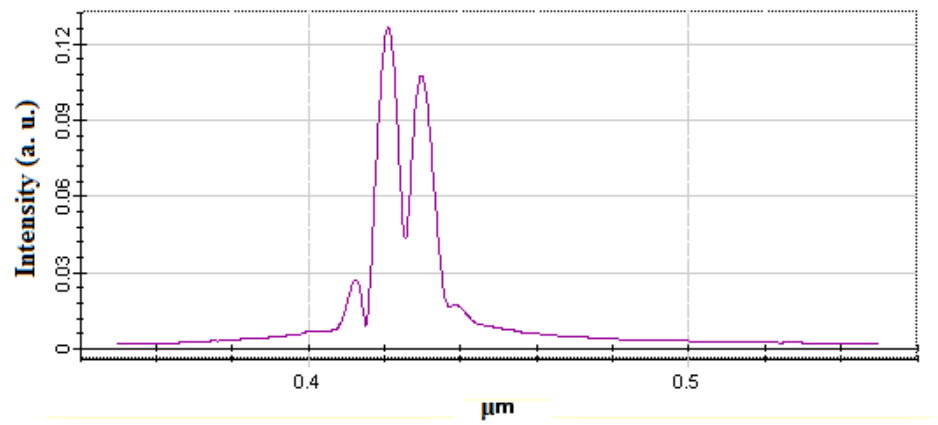

Fig 65 Resonant Wavelength Peaks inside the Microdisk Cavity

Gallium Nitride rings of the same outer radius was also simulated and compared to the disk structure. Due to the hollow structure the reflections from the internal walls of the ring were reduced which reduced the whispering gallery modes and showed mostly the guided wave modes. The ring structure acts as a looped planar waveguide and showed more optical losses as compared to a disk structure. 

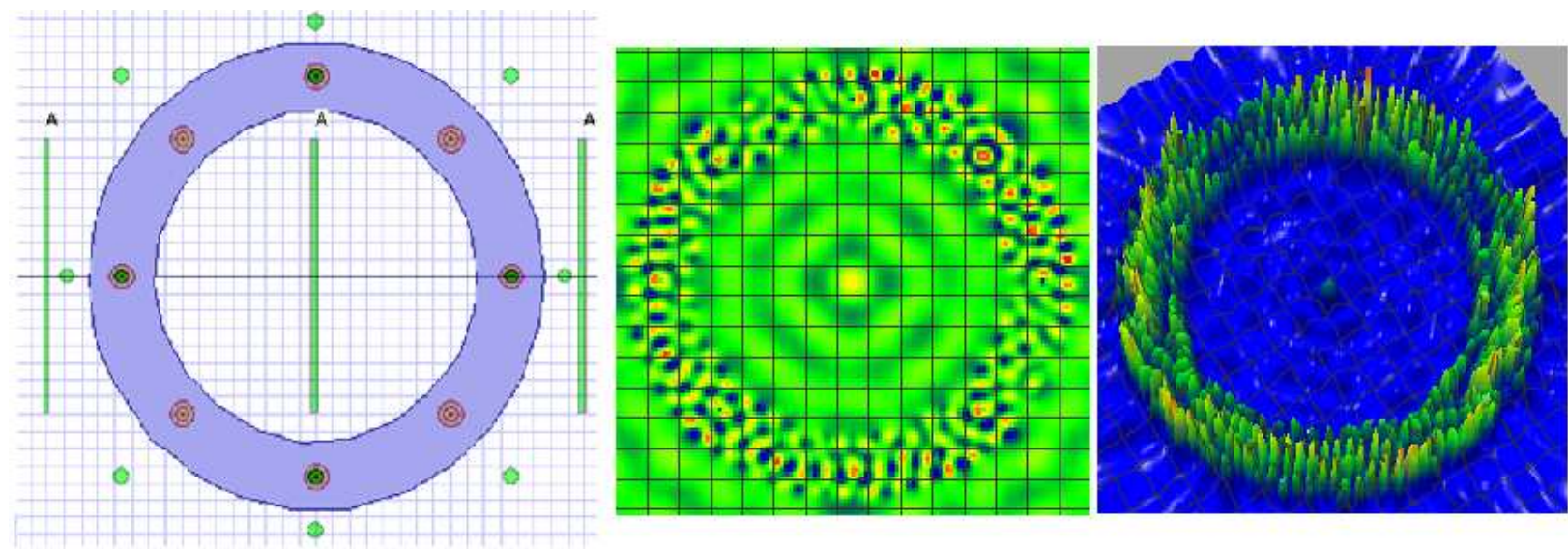

Fig 66 Guided-wave Optical Modes in a Microring Structure

The Microdisk structure has multiple radial whispering gallery modes but the first order fundamental mode is the most sensitive to the refractive index changes on the surface of the disk and can be used for sensing purposes. Therefore calculated positions for point sources were able to excite the first order mode only. The first order WGM is shown in the figure below.
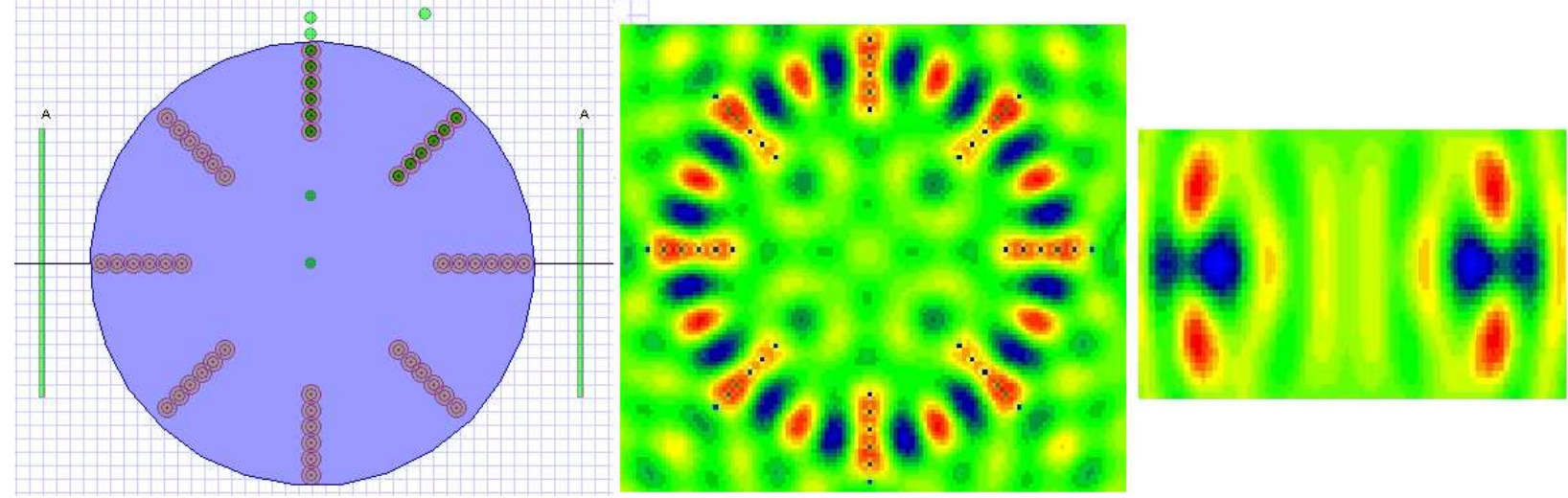

Fig 67 Excitation of First order WGMs (Left) and Side View of Vertical Mode (Right)

The separation between resonant peaks of the whispering gallery, radial and vertical cavity modes can be calculated and resonant peaks can be characterized for mode separation. 

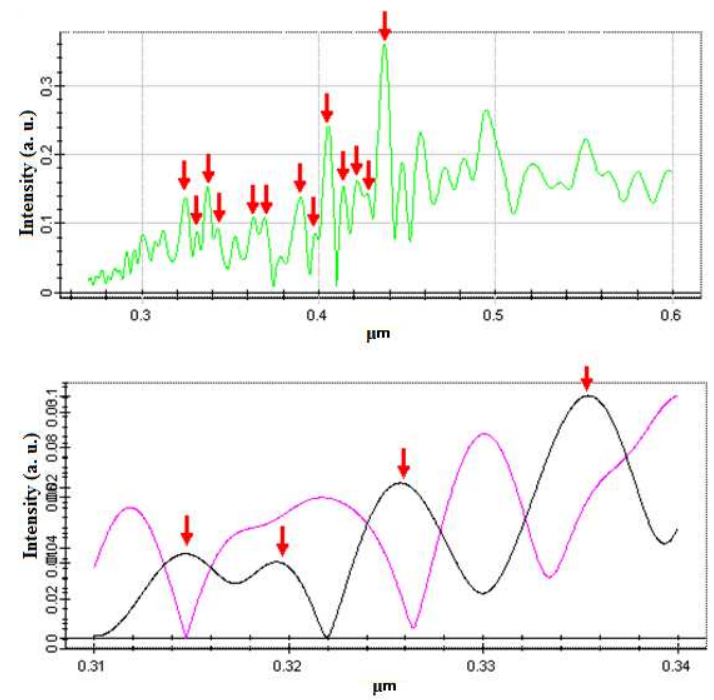

\section{(WGM)}

$\Delta \lambda_{\mathrm{w}}=\lambda^{2} / 2 \pi \mathbf{R n}$

(Radial)

$\Delta \lambda_{\mathbf{r}}=\lambda^{2} / 2 \operatorname{Rn}$

(Vertical Cavity)

$\Delta \lambda_{\mathrm{c}}=\lambda^{2} / \mathbf{L n}$

\section{Fig 68 Separation of Whispering Gallery, Radial and Vertical Cavity Modes}

The Microdisk dimensions can be designed to enhance WGM and reduce the effect of radial and vertical cavity modes or vice versa. A GaN disk on a GaN substrate was simulated to understand the effects of power loss to the substrate and to shift the mode profile towards the disk surface by designing appropriate disk and substrate dimensions. A $600 \mathrm{~nm}$ thick GaN disk on a $1 \mu \mathrm{m}$ thick GaN substrate was used to analyze an uneven WGM mode profile.

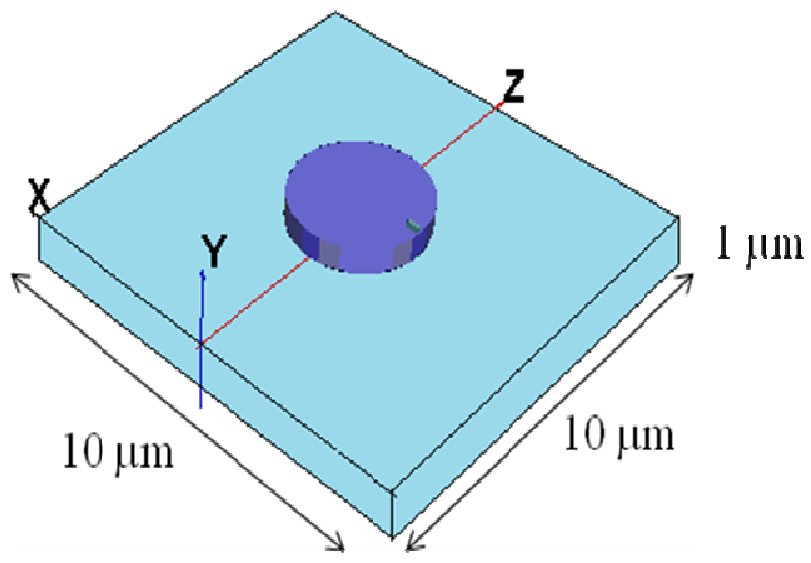

Fig 69 GaN Micro-disk Structure on a GaN Substrate 
The effective refractive index on one side of the disk was made higher (to understand the effect of R.I. variations on WGM) as compared to the other side which creates an asymmetric waveguide profile and changes the effects of WGM on two different sides of the disk as shown in figure below.

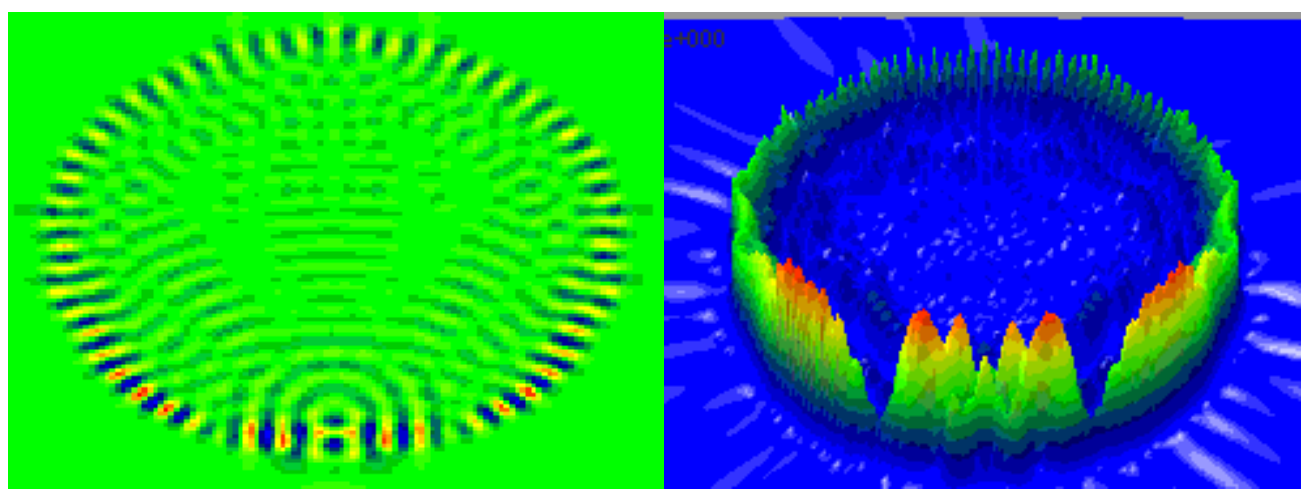

Fig 70 Asymmetric WGM Profile due to Nonlinear Effective Index (Substrate thickness $=1 \mu \mathrm{m})$

Figure 70 shows the WGM mode profile at its peak under the higher index region and dying out eventually on the other side of the disk. Variations of the refractive index of the disk medium and surroundings can be studied to analyze the WGM profile.

Next, the fabrication measures are implemented to improve the sidewall quality of $\mathrm{GaN}$ disks as the roughness greatly scatters the optical power out of the cavity and reduces the effect of any guided wave of whispering gallery modes. The experimentally measured spectral results of the whispering gallery modes will be compared with the simulation results in section 3-3-3. 


\section{3-3-2 Fabrication of GaN Disk Pattern}

Gallium Nitride micro-cylindrical structures were fabricated using standard microfabrication techniques. An un-doped GaN film of $2 \mu \mathrm{m}$ thickness was grown on a sapphire substrate using the MOVPE (metal organic vapor phase epitaxy) method [84]. The fabrication steps are shown in figure 71. E beam lithography technique was used to create PMMA soft masking layer, followed by Nickel evaporation and liftoff. Metal disks are used as a hard mask then to etch $\mathrm{GaN}$ disks. The fabrication details are explained next.

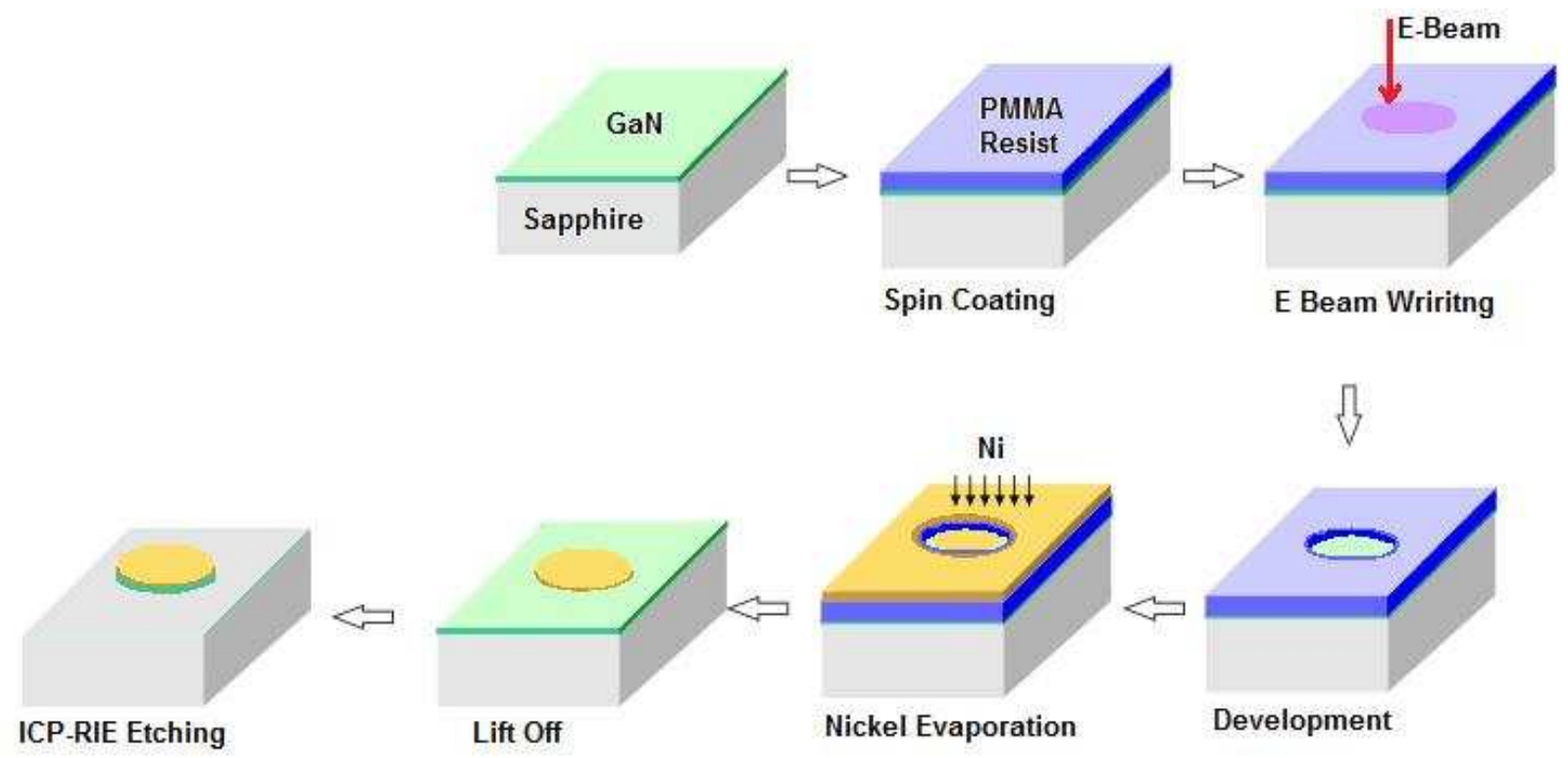

Fig 71 Gallium Nitride Disk Fabrication Steps

\section{3-3-2-1 E-Beam Lithography}

The electron beam lithography transfers a designed pattern from a CAD file to the lithography resist layer (Microchem PMMA A9 950K) (Polymethyl Methacrylate, anisole solvent, 9\% solids, 950,000 molecular weight) coated on the GaN surface. These patterns can eventually be transferred to form structures through various steps of metallization, lift 
off and etching. Photolithography techniques set limits to the resolution of feature sizes and diffraction effects takes place which would create striations in the resist pattern and the GaN disk sidewalls. Lithography resists are photon or electron sensitive, so their chemical properties changes when they are exposed to certain dose of photons or electrons. Therefore exposed parts are either developed away (Positive Resist) or stay intact (Negative Resist). The GaN samples were cleaned in acetone, methanol and DI water for 5 min. each and then blown dry with nitrogen gas. The samples were dried in the oven for $10 \mathrm{~min}$ at $120^{\circ} \mathrm{C}$. The PMMA resist was spun on the samples at 500 RPM for $5 \mathrm{sec}$., 0 RPM for $10 \mathrm{sec}$, and 5000 RPM for 50 seconds. The final resist thickness was measured to be between $0.9 \mu \mathrm{m}$ to 1 $\mu \mathrm{m}$. The samples were then pre-baked on a hot plate for 90 seconds at $180^{\circ} \mathrm{C}$. The e-beam lithography was performed using a scanning electron microscope (SEM) at an electron beam energy of $30 \mathrm{keV}$ and probe current of $40 \mathrm{pA}$, which scans the beam at a controlled rate over the PMMA resist. The electron beam column consists of an electron source, electron lenses (mechanism for beam deflection), beam blanker to turn the beam on or off, astigmator to correct astigmatism, aperture, alignment systems and electron detector to help focus and locating marks on the sample. Figure 72 shows the E-beam SEM used for this dissertation work. 


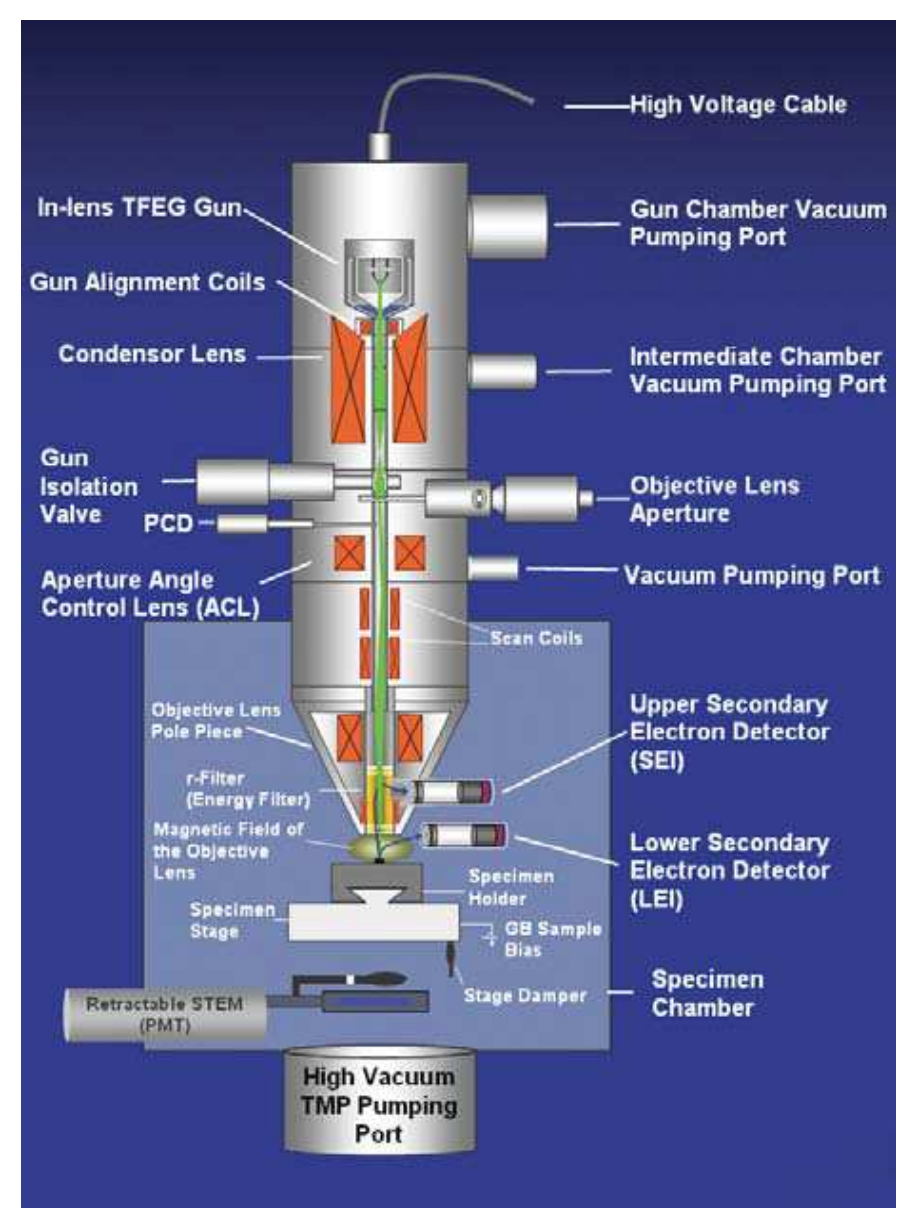

\section{Fig 72 Scanning Electron Microscope Components [85]}

The electron beam is generated from a conducting material by heating (thermionic emission) or applying electric field (field emission) enough to increase the electron energy above the work function of the conductor. The current fluctuation is minimized by operating the electron source in a ultra high vacumm (UHV) chamber with pressure upto $1.9 \mathrm{e}-4$ torr or better. The electron lenses focus the beam using electric or magnetic forces. As the primary electrons penetrate the PMMA resist layer, forward angular scattering occurs which broadens the beam diameter in the resist. By the time electrons reach the substrate (secondary electrons) they undergo large angular scattering back into the resist known as back scattering. Due to this back scattering, 'Proximity Effect' takes place, where the dose of electrons received by the resist features is affected. The main drawback of the proximity effect is that it broadens the beam diameter by $10 \mathrm{~nm}$, which changes the actual dose of 
exposure. The minimum practical resolution of $20 \mathrm{~nm}$ is achieved with a beam center to center spacing of $6.49 \mathrm{~nm}$. The beam broadening also causes 'Bias Effect' where the exposed features develop bigger than they were normally written. Hence the dose is over or under-exposed. Therefore a proper adjustement is required to the dose, by writing the pattern multiple times, until the pattern comes out the proper size. Higher beam voltages reduce these scattering effects and improve the beam range. The best area dose of e-beam developed for a Micro-chem (positive resist) PMMA (A9-950K) of $1 \mu \mathrm{m}$ thickness coated on $\mathrm{GaN}$ samples was found to be within $350-400 \mu \mathrm{C} / \mathrm{cm}^{2}$.

$$
\text { Dose } \times \text { Area }=\text { Exposure Time } \times \text { Current }
$$

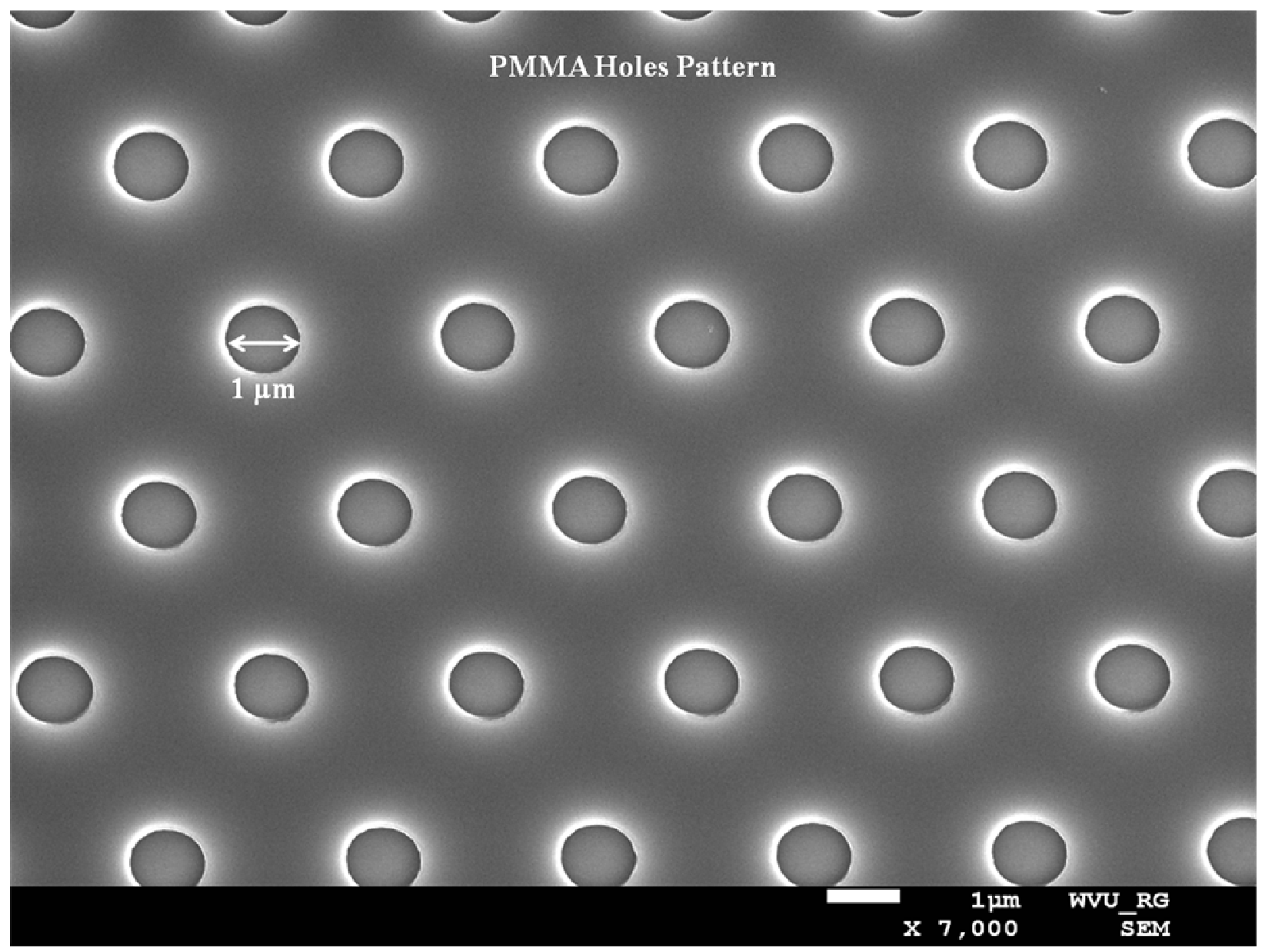

Fig 73 Developed PMMA Holes Pattern on GaN sample (Dose: $400 \mu \mathrm{C} / \mathrm{cm}^{2}$ ) 

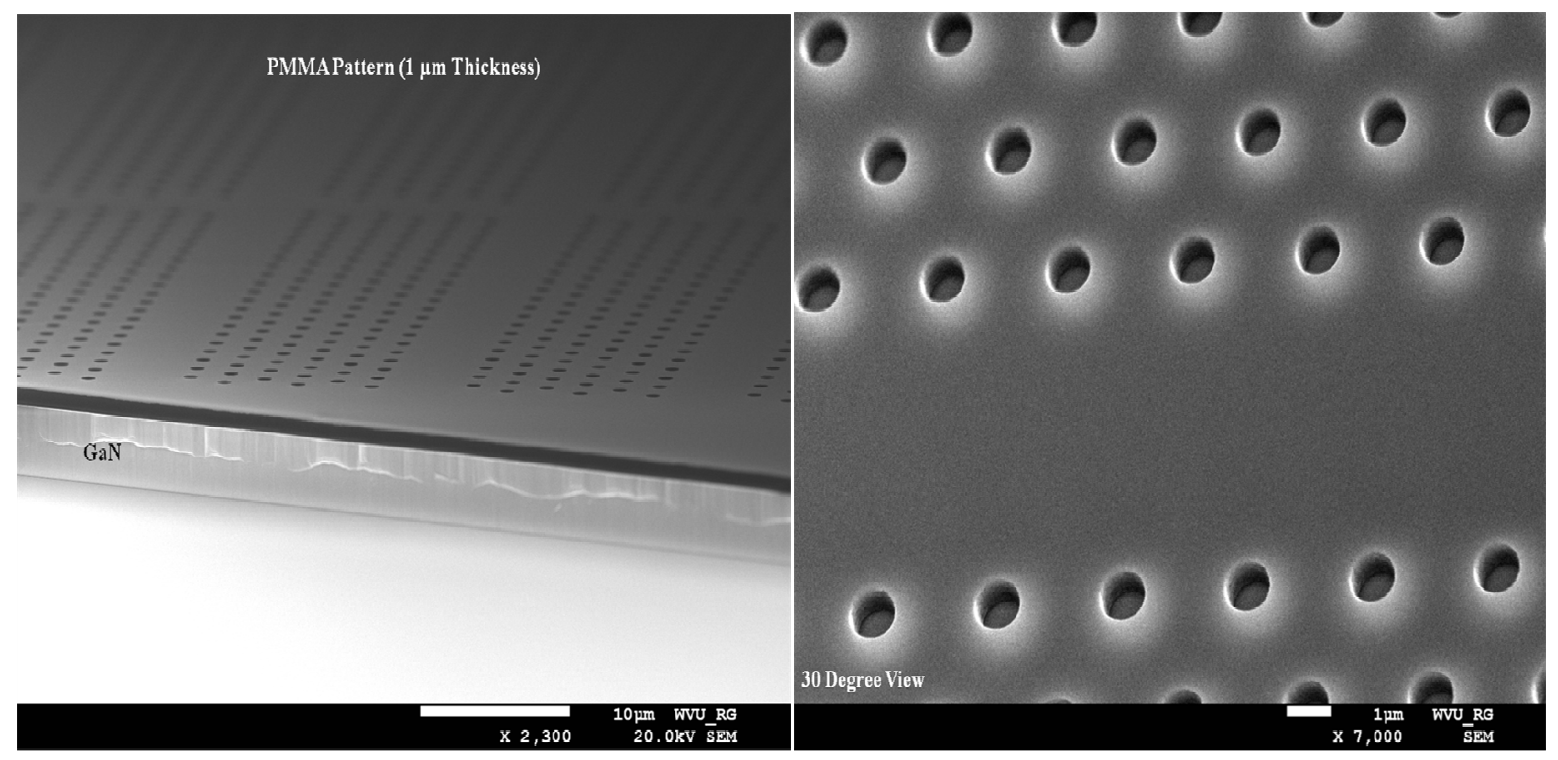

Fig 74 Developed PMMA Holes Pattern on GaN sample (Tilted View)

Various patterns of disk radius $500 \mathrm{~nm}, 2 \mu \mathrm{m}, 2.5 \mu \mathrm{m}, 3.5 \mu \mathrm{m}$ and $4.5 \mu \mathrm{m}$ were developed. The minimum disk edge to edge spacing was kept at $1 \mu \mathrm{m}$ with total area of each pattern to be $250 \times 250 \mu \mathrm{m}^{2}$. The pattern were developed in a 1:3 MIBK:IPA developer for 70 seconds without agitation. The sample was dipped in IPA solution for 15 secnds to stop development, then cleaned with DI water and blown dry with nitrogen. 


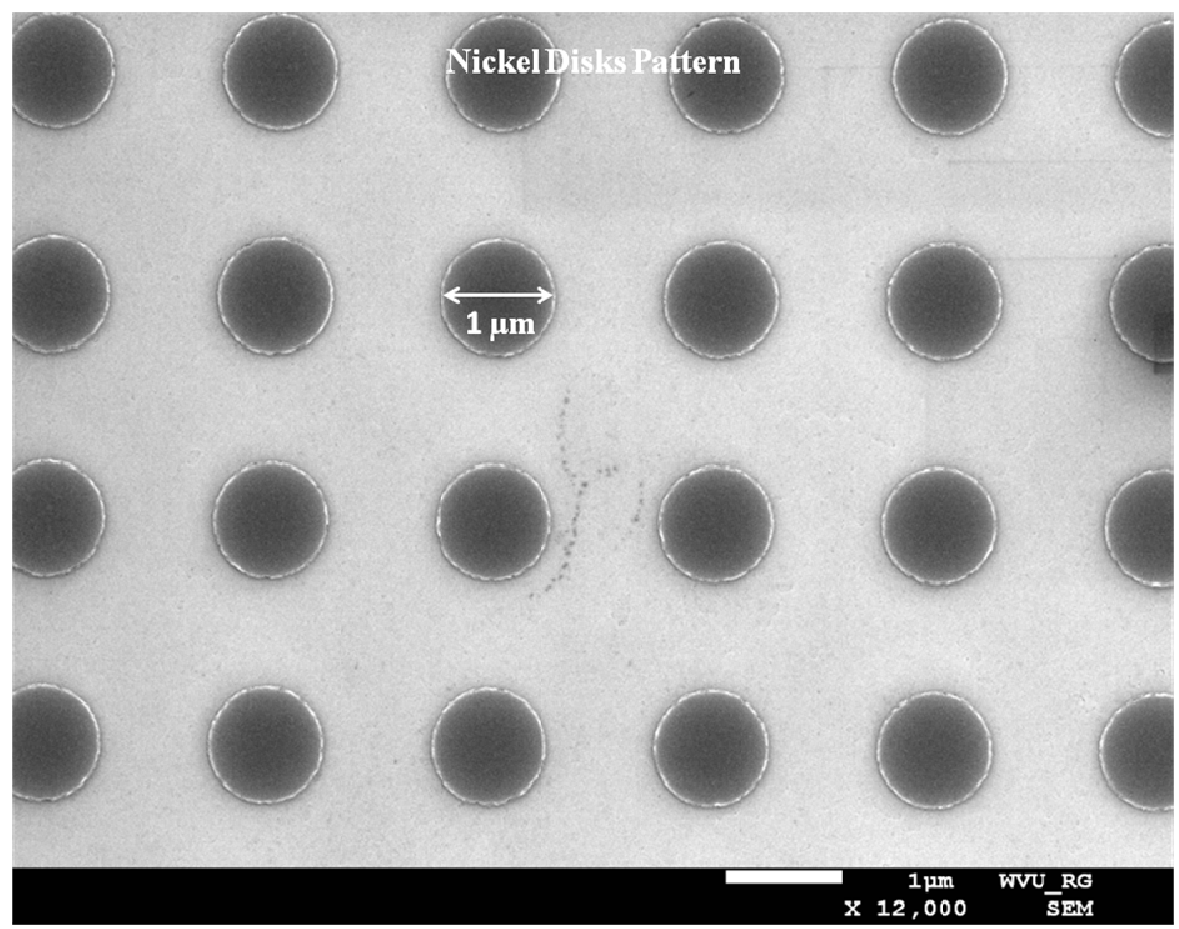

Fig 75 Nickel Disks as Hard Mask on GaN sample (After PMMA Lift off)

E-beam evaporation (Temescal BJD 2000) was used to deposit Nickel (100-150 $\mathrm{nm})$ at a rate of $1 \AA / \mathrm{sec}$. with a density of $8.91 \mathrm{~g} / \mathrm{cc}$ and a Z-ratio of 0.331 . Zero dome rotation enhanced the deposition unifromity and a better sidewall profile. The PMMA was then lifted off using PG remover for 30 minutes at $65^{\circ} \mathrm{C}$. The nickel disk pattern on GaN samples after PMMA lift off is shown in the figure above. The samples were ready for ICPRIE etching as discussed in the next section.

\section{3-3-2-2 ICP-RIE Etching}

The e beam lithography defines a pattern on the GaN samples, which can be used as a hard mask for etching and to transfer the pattern as GaN disks. The inductively coupled plasma (ICP) reactive ion etching (RIE) is a dry etching technique which involves gasphase etchants in plasma. A combination of chemical and physical processes takes place to define parameters like etch selectivity and directionality. Etch selectivity is the ratio of the 
etch rates of different materials in an etch process. The GaN: Ni etch selectivity using a (Trion Mini-lock III) etching system was found to be 8:1. Etch directionality measures the relative etch rate in different directions, usually vertical versus lateral. Isotropic etching occurs when etch rates are the same in all directions, whereas Anisotropic etching etches in one direction. Etch directionality is related to physical effects such as ion bombardment and sputtering. Vertical etching with a smooth sidewall is the focus in this dissertation. The etch chemistry was selected based on the directionality as well as smoothness of etched sidewalls.

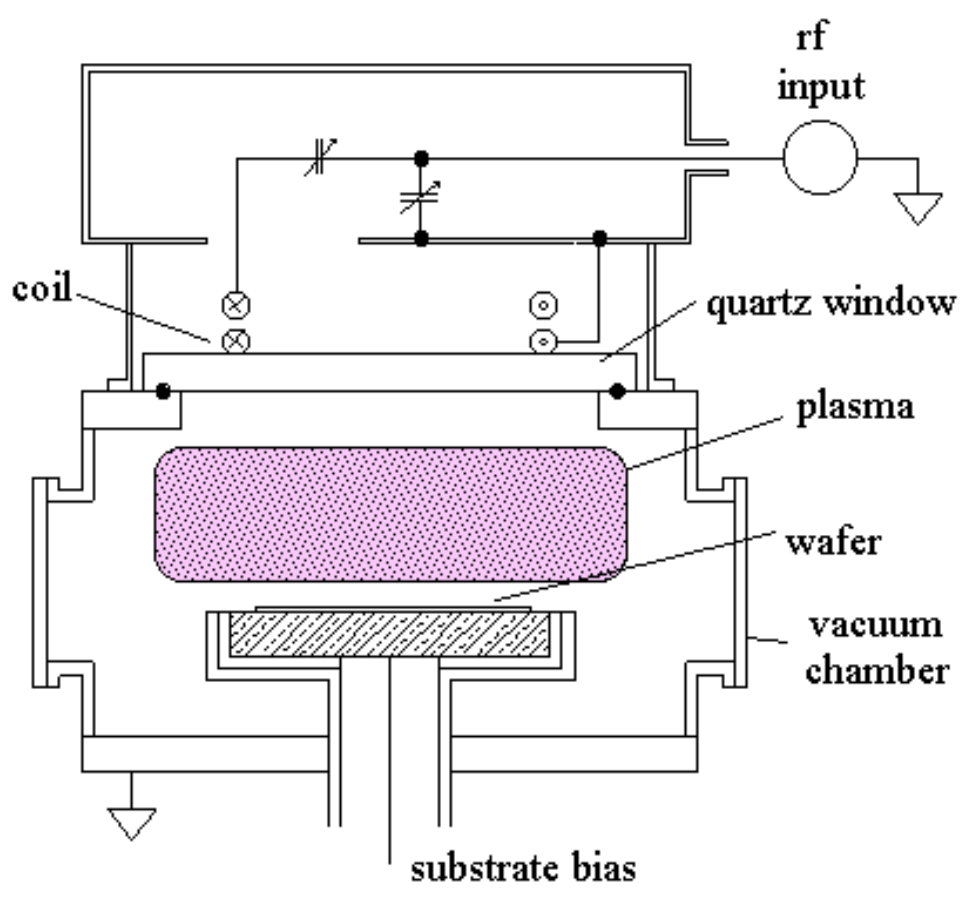

Fig 76 A Basic Plasma Etch System Schematic [86]

The chamber pressure of $10 \mathrm{mTorr}$ was used, and by applying a high electric field (ICP Power) across two electrodes, some of the gas atoms are ionized, producing ions, electrons and neutrals which form the plasma. The energy is supplied by a RF generator typically at $13.5 \mathrm{MHz}$, and due to difference in the mobility of electrons, positive ions and neutrals, a voltage bias is developed between the plasma and the electrodes. The plasma density (concentration of ions) and ion energy are coupled. As the RF power increases, 
plasma density is increased, and voltage bias increases, increasing the ion energy and the etch rates. For the RIE system the upper electrode is made bigger, which results in a larger voltage drop from plasma to the wafer, resulting in more energetic ion bombardment, which improves the directionality. Increasing the RF power or voltage drop too much can also cause wafer damage, therefore a recipe is created to achieve the target etching rate, smoothness and directionality. To achieve smoothness the DC Bias was kept lower ( $\sim 55 \mathrm{~V})$, which reduces the ion energy and wafer damage, and also improves the selectivity. The two species in the plasma used for etching are neutral chemical species $\left(\mathrm{Cl}_{2}\right)$, which etches the material chemically, and the positive ions $\left(\mathrm{BCl}_{3}\right)$ used for physical etching. The purpose of reactive neutral chemical species is to react with $\mathrm{GaN}$ and etch, with a volatile by product. The positive ions are accelerated towards the wafer due to a voltage drop between the plasma and the wafer, resulting in a more physical component of etching. The physical etching breaks bonds and makes material more prone to chemical reactions. However, the physical etching is more directional as compared to the reactive neutral chemical etching but also can cause wafer damage. The physical and chemical by products were removed using the gas outlet pump. The etching recipe used for $\mathrm{GaN}$ was $\left(\mathrm{BCl}_{3}: \mathrm{Cl}_{2}\right)(6: 34) \mathrm{sccm}$, at a chamber pressure of $10 \mathrm{mTorr}$, ICP power (Plasma Density) of $400 \mathrm{~W}$, RIE power (Ion Energy) of $150 \mathrm{~W}$ and a DC Bias voltage of $-85 \mathrm{~V}$. The samples were etched for 10 minutes to completely remove the Nickel mask $(100 \mathrm{~nm})$ creating a GaN disk MESA of $\sim 800 \mathrm{~nm}$ as shown in the figure below. Nickel etch rates were observed to be $10 \mathrm{~nm} / \mathrm{min}$. and $\mathrm{GaN}$ etch rates were $80 \mathrm{~nm} / \mathrm{min}$. A higher DC Bias of $-150 \mathrm{~V}$ bombards the sidewalls and generates damaged sidewalls. 


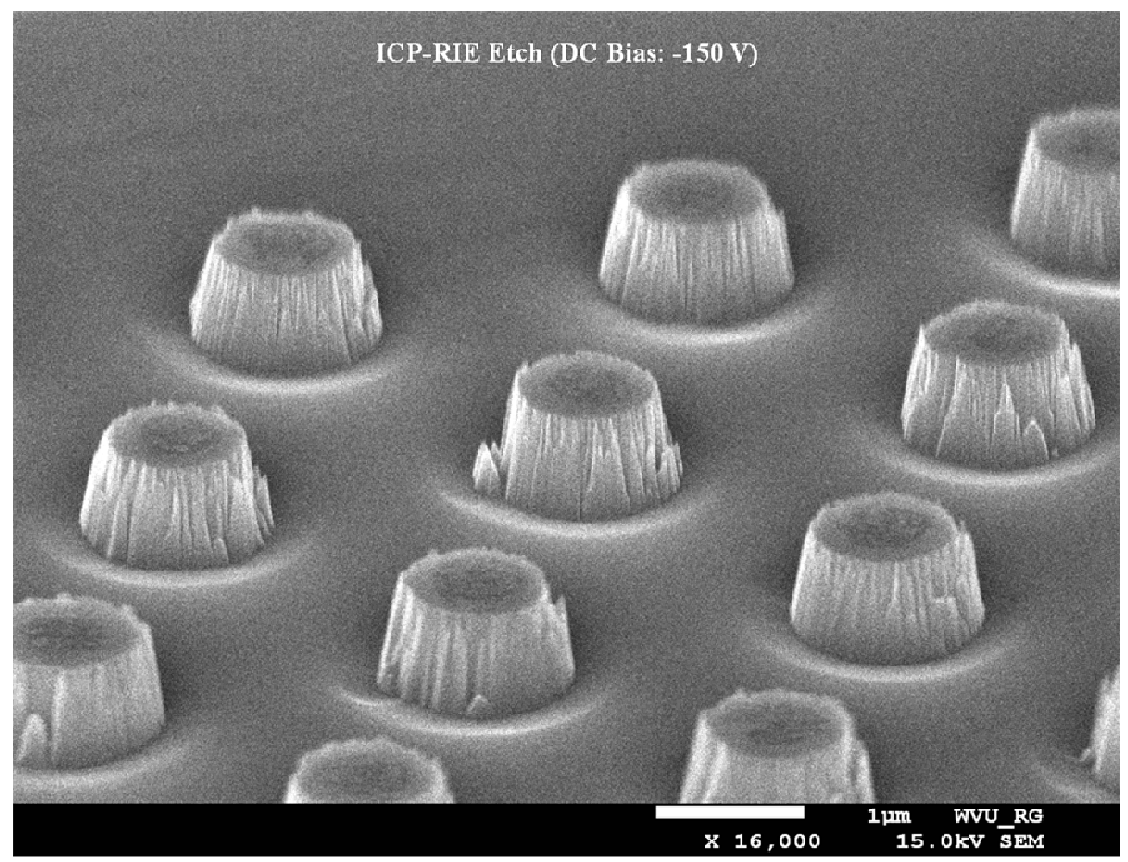

Fig 77 GaN Disk Pattern at a Higher DC Bias Voltage of -150 V Showing Ion Damage to Sidewall

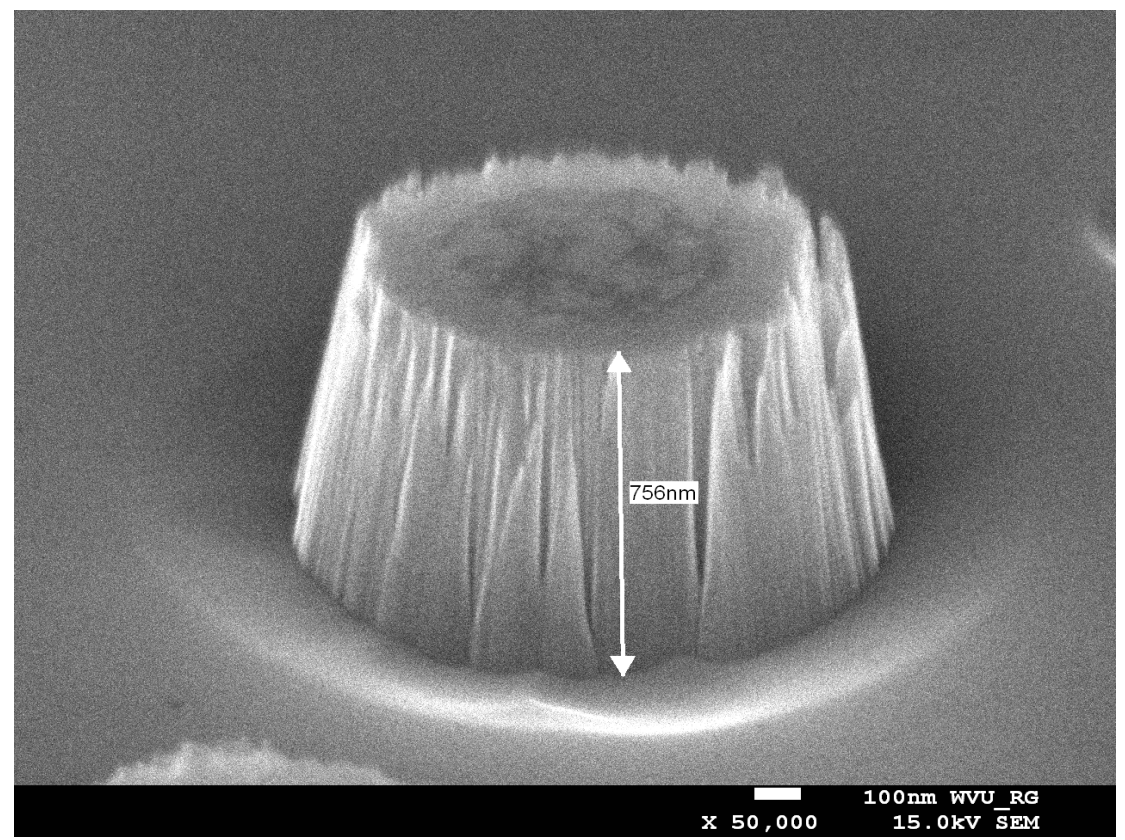

Fig 78756 nm Thick GaN Disk with Damaged Sidewall

As the DC Bias is reduced to $-85 \mathrm{~V}$, the ion energy is reduced, and sidewall quality 
is improved as seen in the figure below.
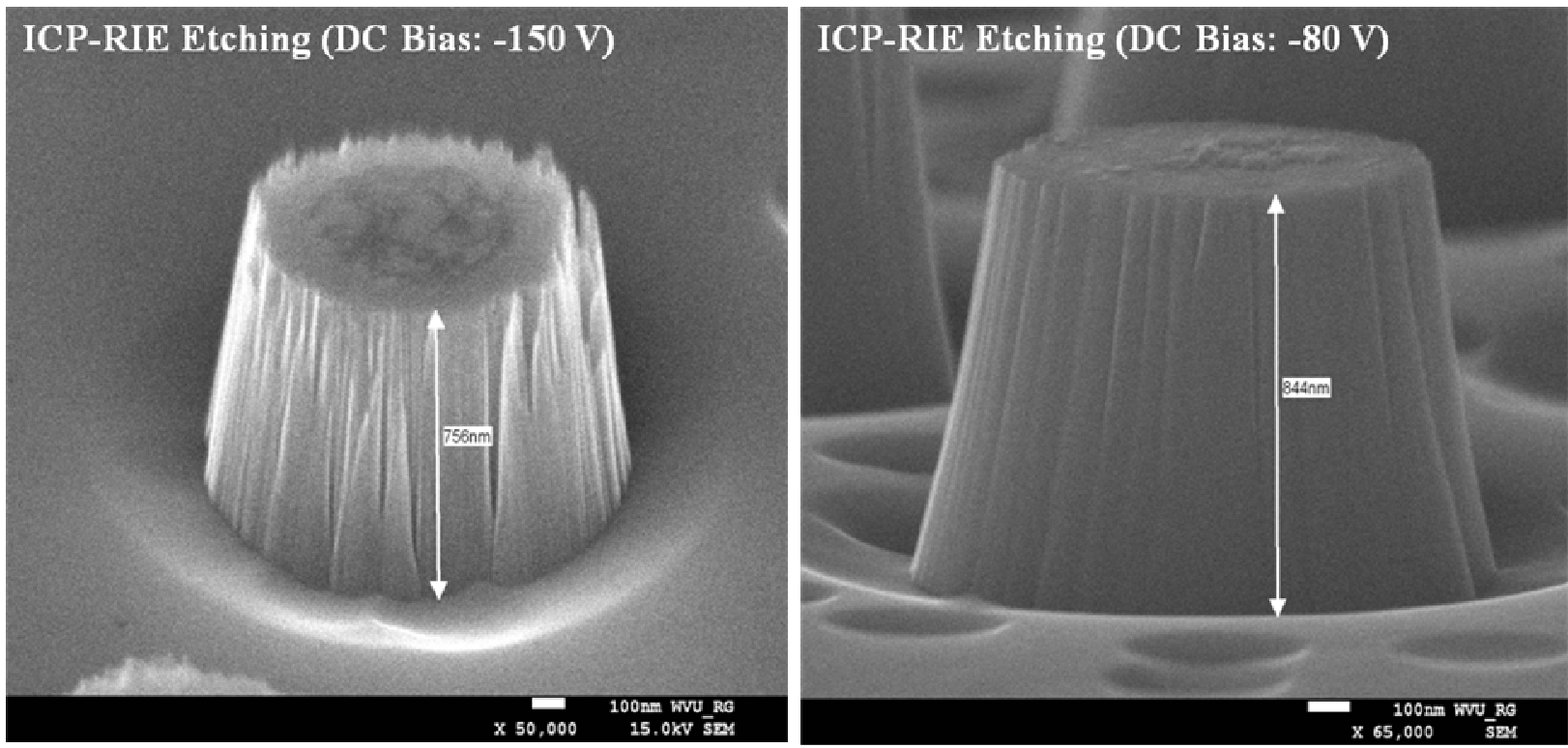

Fig 79 GaN Disks Sidewall Quality Improvement with Lower DC Bias

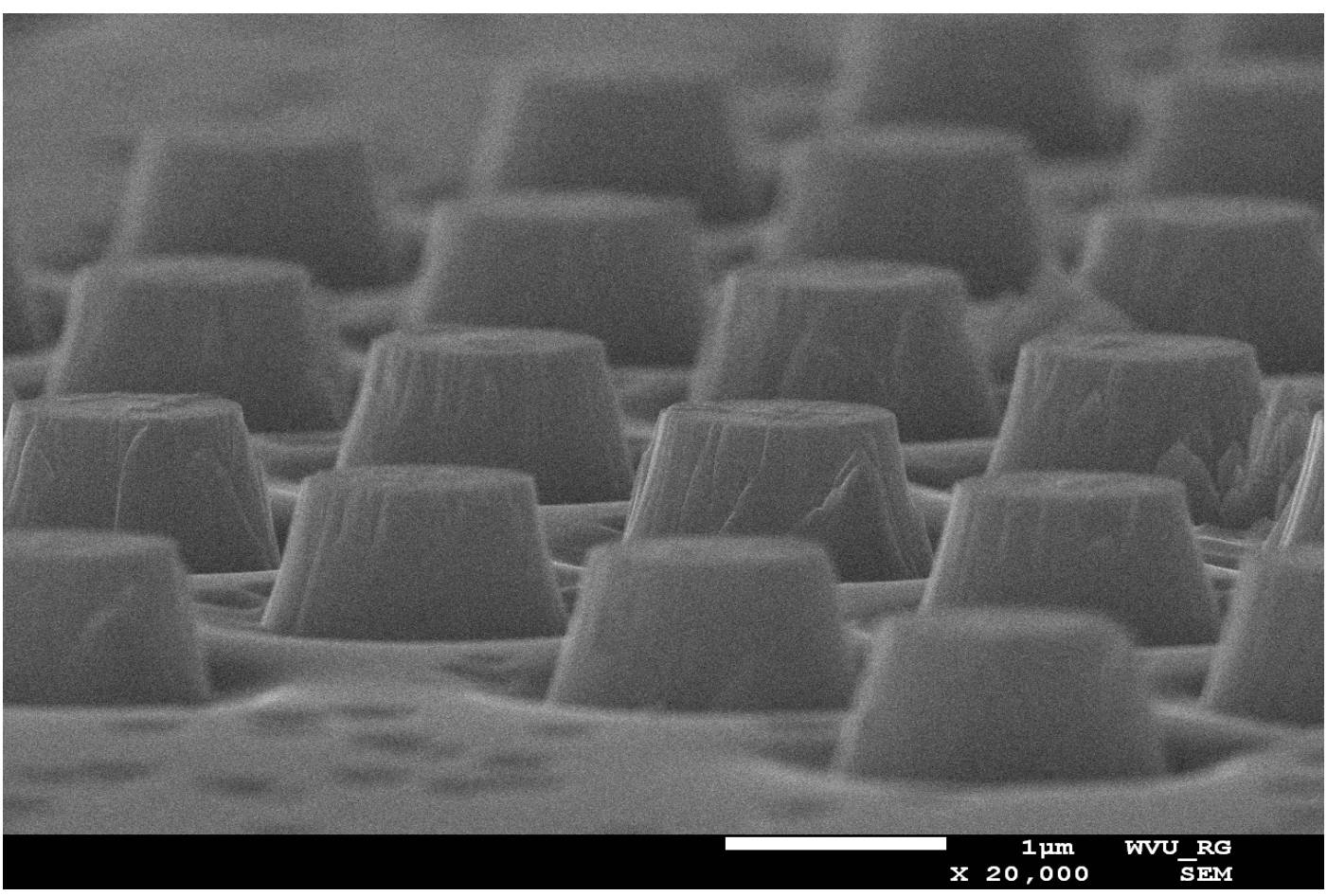

Fig 80 GaN Disks Pattern 
The sidewall angles were measured to be 75 degrees. For whispering gallery modes generation in GaN disks, the sidewall quality was emphasized as compared to the sidewall angles. The disk structures were circular trapezoidal in shape, with smoother sidewalls to prevent scattering of light at the edges.

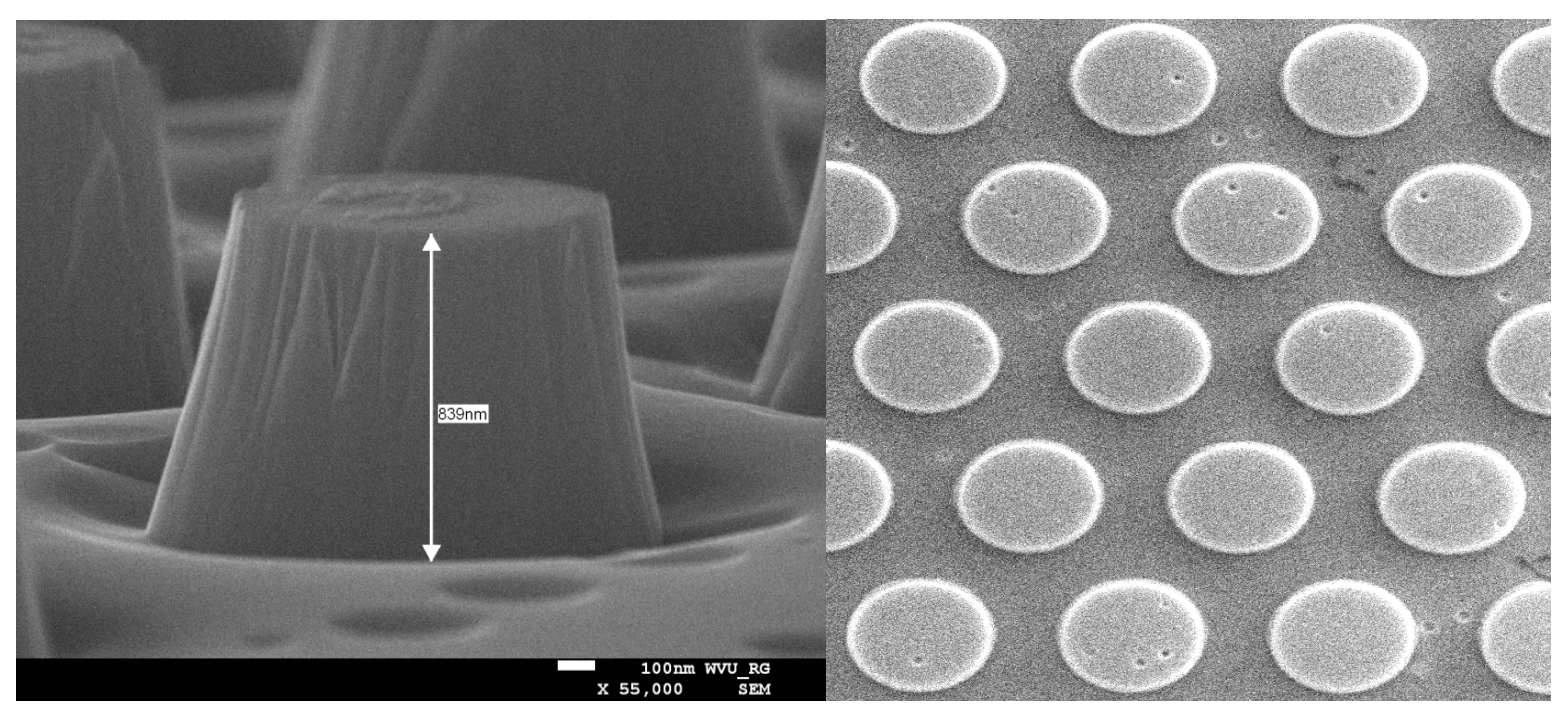

Fig 81 Side View and Top View of GaN Disks Pattern

The GaN disks pattern were fabricated using e-beam lithography and ICP-RIE etching techniques. The samples with disks of various radii were characterized optically for detection of whispering gallery modes as discussed in the next section.

\section{3-3-3 Optical Characterization of Whispering Gallery Modes}

The higher index GaN disks on lower index sapphire substrates were designed with sidewalls thicknesses, so that the optical mode propagates in the disks, and optical losses into the substrate can be prevented. The Opti-BPM simulation tool was used to solve for optical modes. The disks on the sapphire substrate can be simulated as a ridge waveguide with optical mode propagating in the GaN disks as shown in the simulated plot below. 


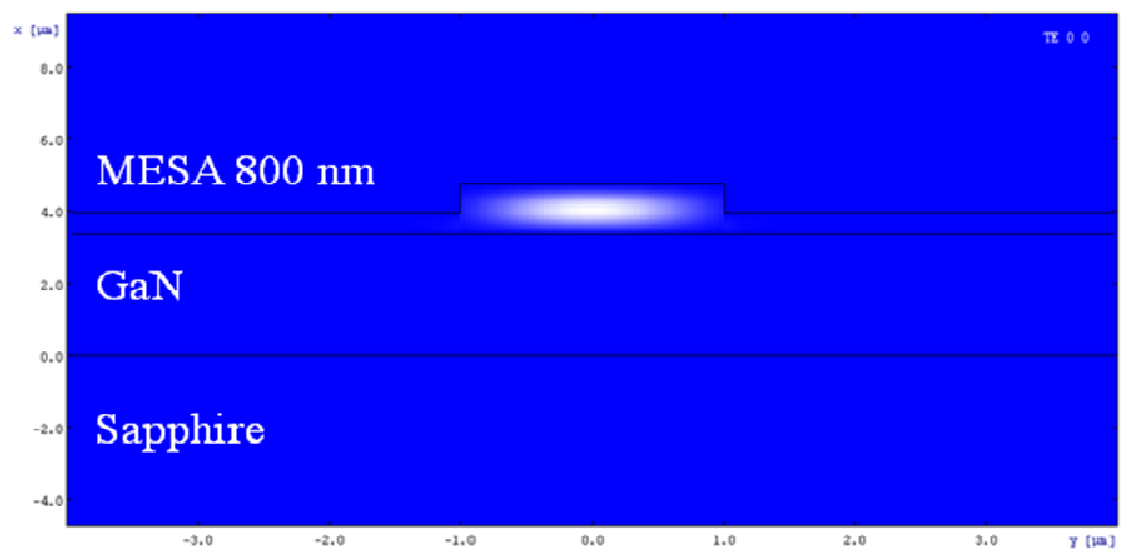

Fig 82 Optical Mode Propagation in GaN Disk $(800 \mathrm{~nm})$ Simulated as a Ridge Waveguide Structure

The optical coupling into the disks was achieved using a RadioShack blue LED with a peak wavelength of $463 \mathrm{~nm}$ (FWHM $30 \mathrm{~nm}$ ), optical lenses, microscope objective (10x), BWTEK optical fiber (200 $\mu \mathrm{m}$ core diameter) and a BWTEK UV-NIR spectrometer (0.8 $\mathrm{nm}$ spectral resolution). The optical bench coupling setup is shown in the figure below.

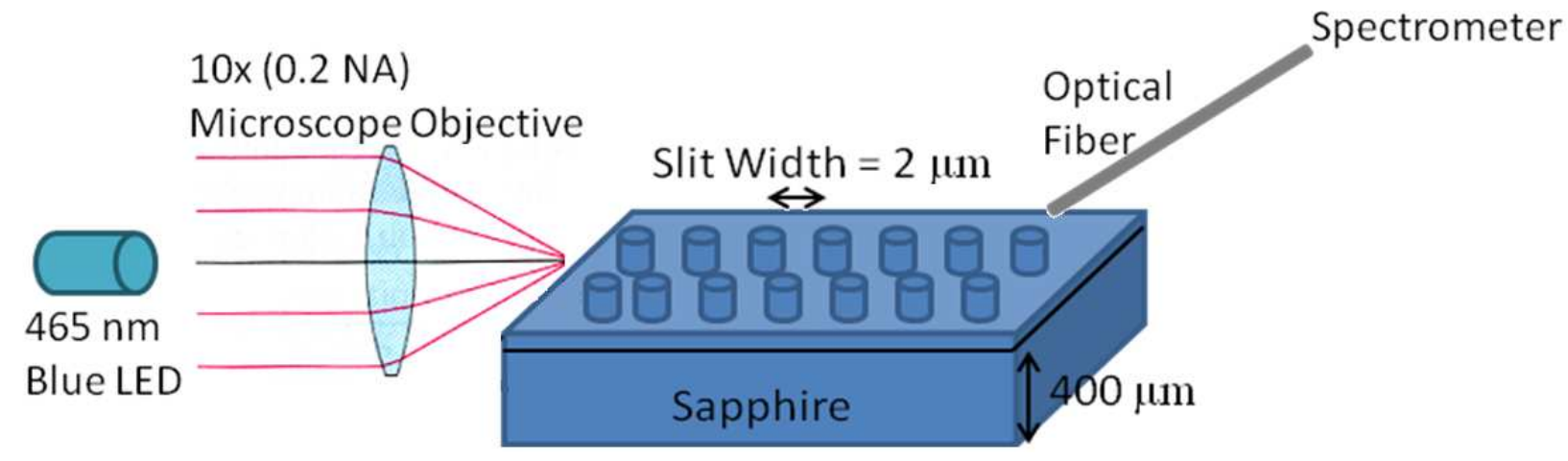

Fig 83 Optical Setup for WGM Coupling in GaN Disk Pattern

The high intensity output blue LED was coupled with a microscope objective to focus the light beam on the disk array. To couple light into the whispering gallery modes of the disk, and prevent any noise from the diffraction grating spectra (generally seen at $45^{\circ}$ ), the input light beam was coupled at a $10^{\circ}$ angle from the substrate surface. The output spectrum was collected at $20^{\circ}$ from substrate surface using a focusing lens, and aligned with 
the optical fiber. The whispering gallery modes of $\mathrm{GaN}$ disks $(n=2.45)$ correspond to the spectral minima at the output, and having a mode spacing which is defined by this relation:

$$
\Delta \lambda=\frac{\lambda^{2}}{2 \pi R n}
$$

GaN disks of radii $2 \mu \mathrm{m}, 3.5 \mu \mathrm{m}$ and $4.5 \mu \mathrm{m}$ were optically characterized with a mode spacing of $7 \mathrm{~nm}, 4 \mathrm{~nm}$ and $3 \mathrm{~nm}$ respectively, which satisfied the relation in equation 3.12. The WGM experimental spectra can be seen in the plots below.

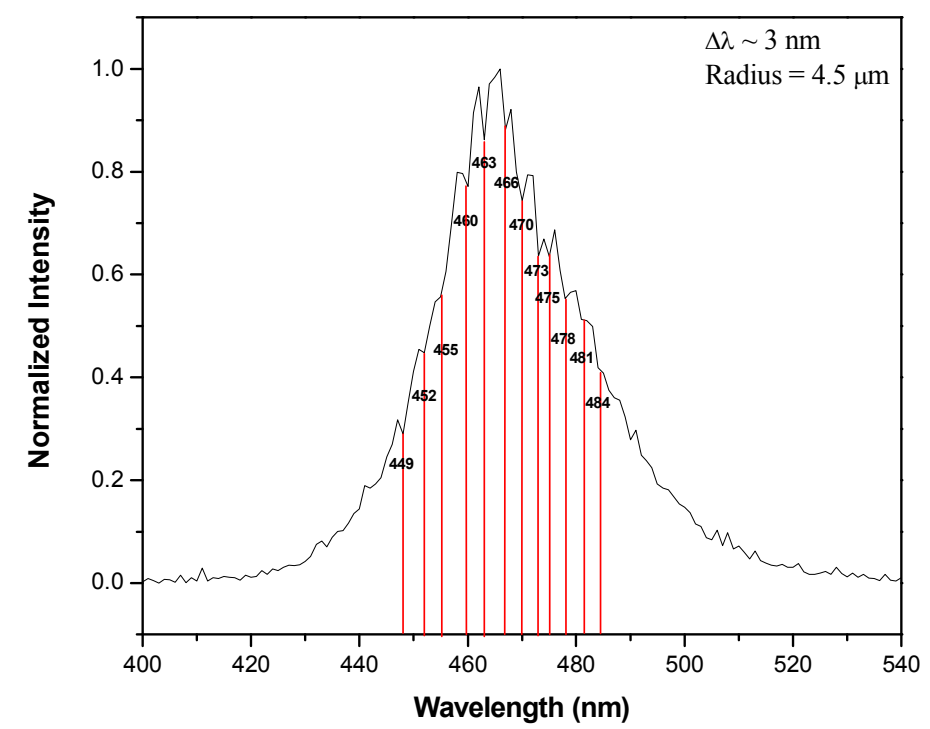

Fig 84 Whispering Gallery Modes as Spectral Minima for $4.5 \mu \mathrm{m}$ Radius Disk 


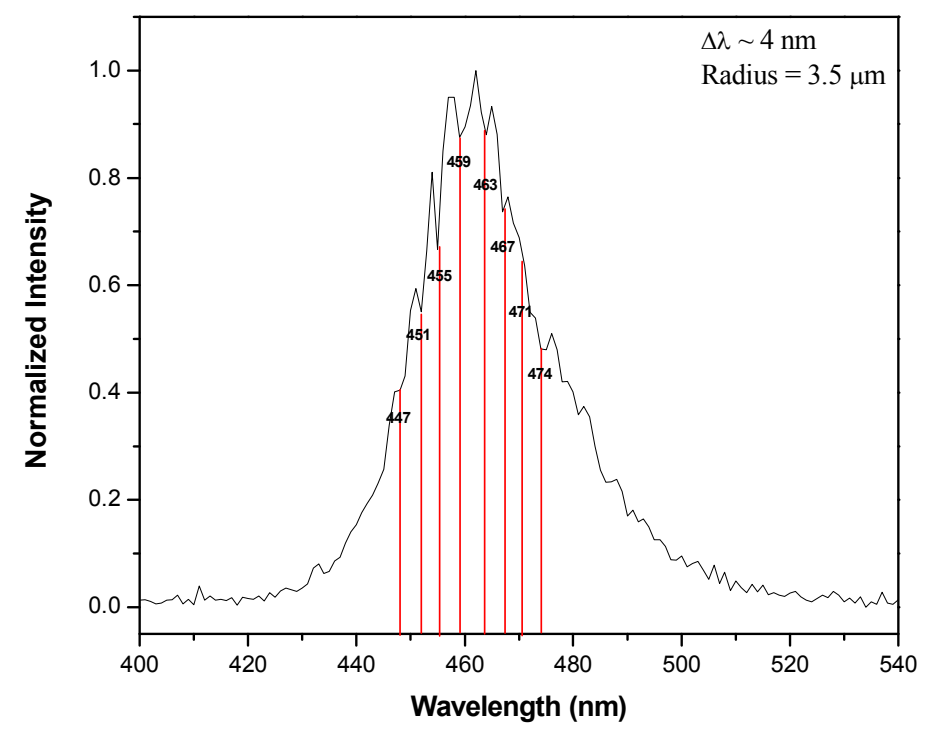

Fig 85 Whispering Gallery Modes as Spectral Minima for $3.5 \mu \mathrm{m}$ Radius Disk

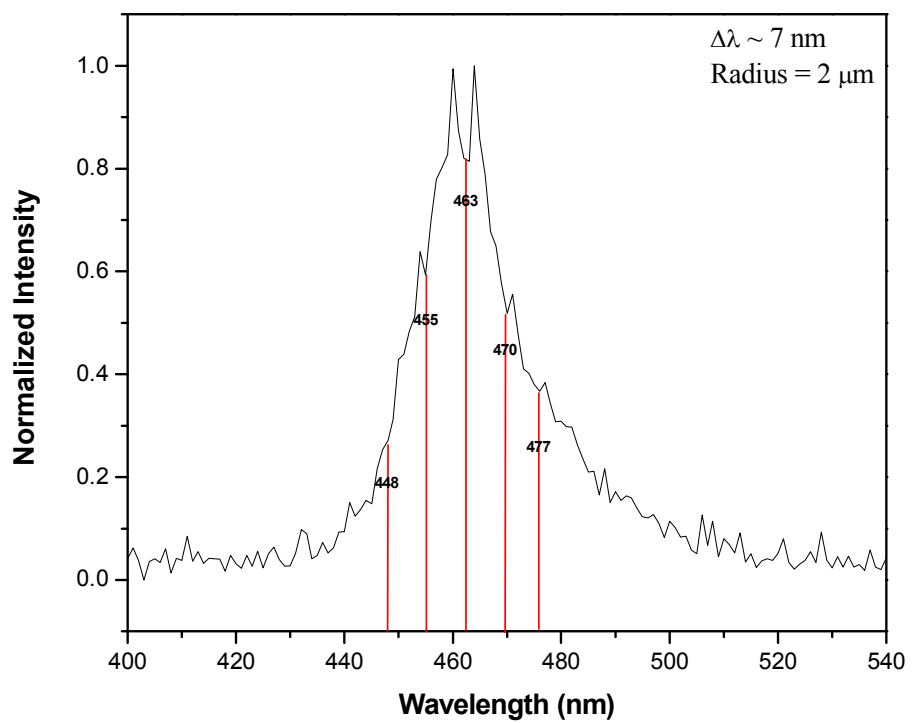

Fig 86 Whispering Gallery Modes as Spectral Minima for $2 \mu \mathrm{m}$ Radius Disk 


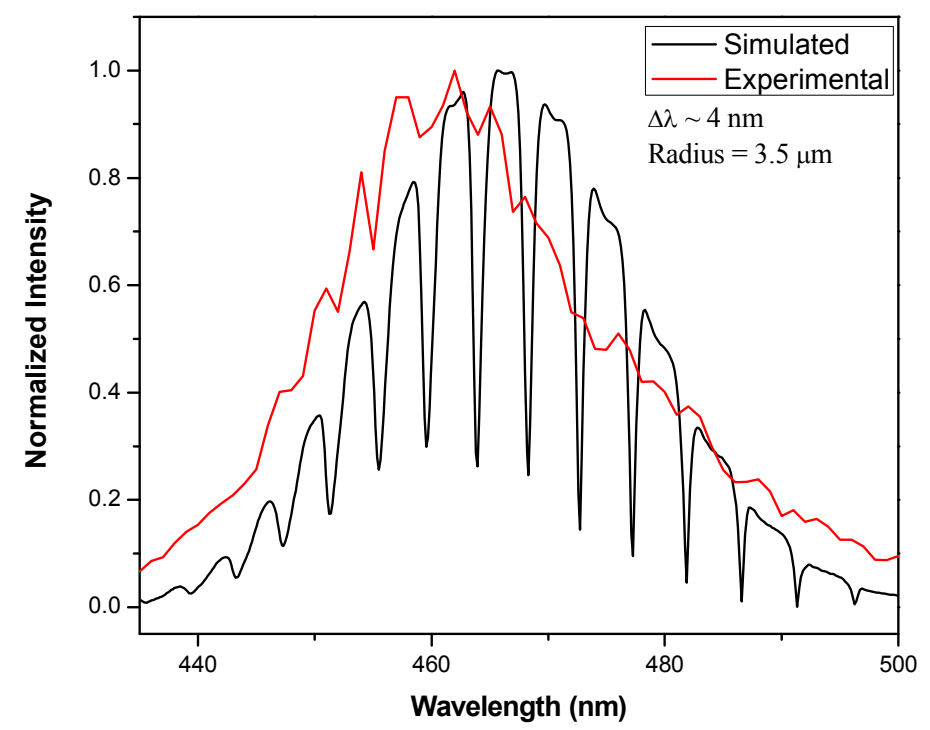

Fig 87 Matched Experimental and Simulated WGM Spectra for 3.5 $\mu$ m Radius Disk

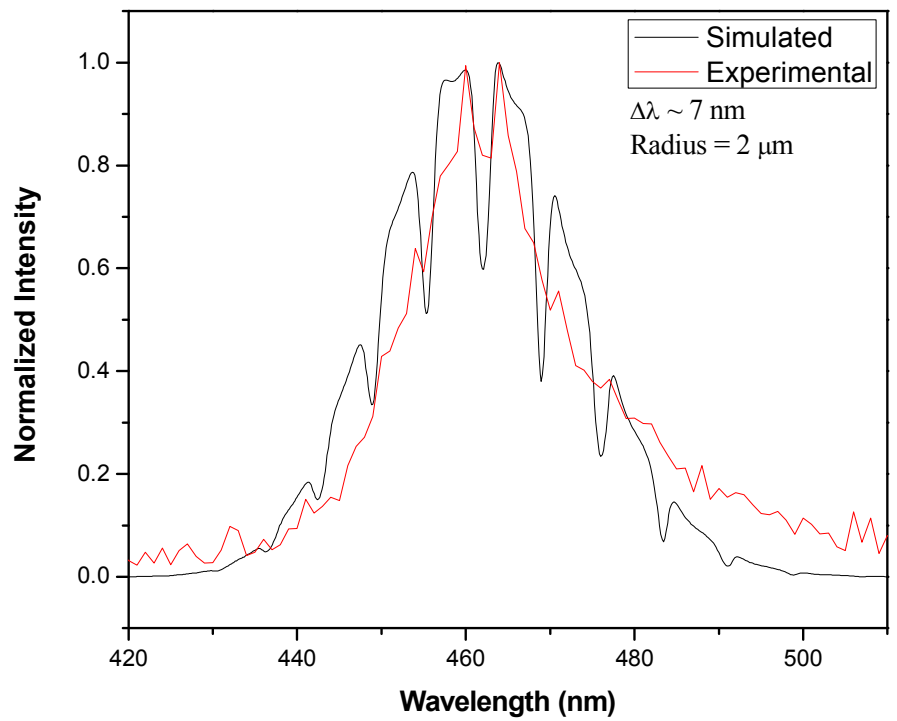

Fig 88 Matched Experimental and Simulated WGM Spectra for $2 \mu \mathrm{m}$ Radius Disk

According to relation 3.12, the decrease in radius of the disk will increase the mode 
spacing, (as a shorter path length will fit fewer resonance wavelengths) and was confirmed using simulations and experimentally. The GaN disk gratings with a 'center to center' distance of $3 \mu \mathrm{m}$ produced a grating resonance $(d \sin \theta=m \lambda)$ of $170 \mathrm{~nm}$ separation and were present outside the LED spectrum. The vertical cavity mode spacing was also calculated to be $29 \mathrm{~nm}$, which is similar to the FWHM of the LED source and most of the VCMs were present outside the LED spectrum. Therefore the spectral minima in the experimental and simulated plots were confirmed to be the presence of whispering gallery modes.

\section{3-4 GaN LED Simulations}

The finite difference time domain tool has been established as an important tool for nano photonics simulations. We used the software to model a variety of optical propagation phenomena in planar resonant waveguides, micro-spherical and micro-cylindrical structures for scattering, diffraction, reflection, refraction, absorption, gain, polarization and near field effects. The material anisotropy and non-linearity can also be modeled without any preassumptions for the optical field and its behavior. As seen in the previous sections the modeling was performed using an external plane wave light source for passive characterization of optical modes. In this section we will simulate GaN LED structures as an active (Internally excited) micro-resonator for analysis of vertical cavity modes (VCMs) and comparison of experimental and simulated LED EL (electroluminescence) spectra. A typical GaN LED structure is shown below. 


\section{GaN Disk LED Structure}

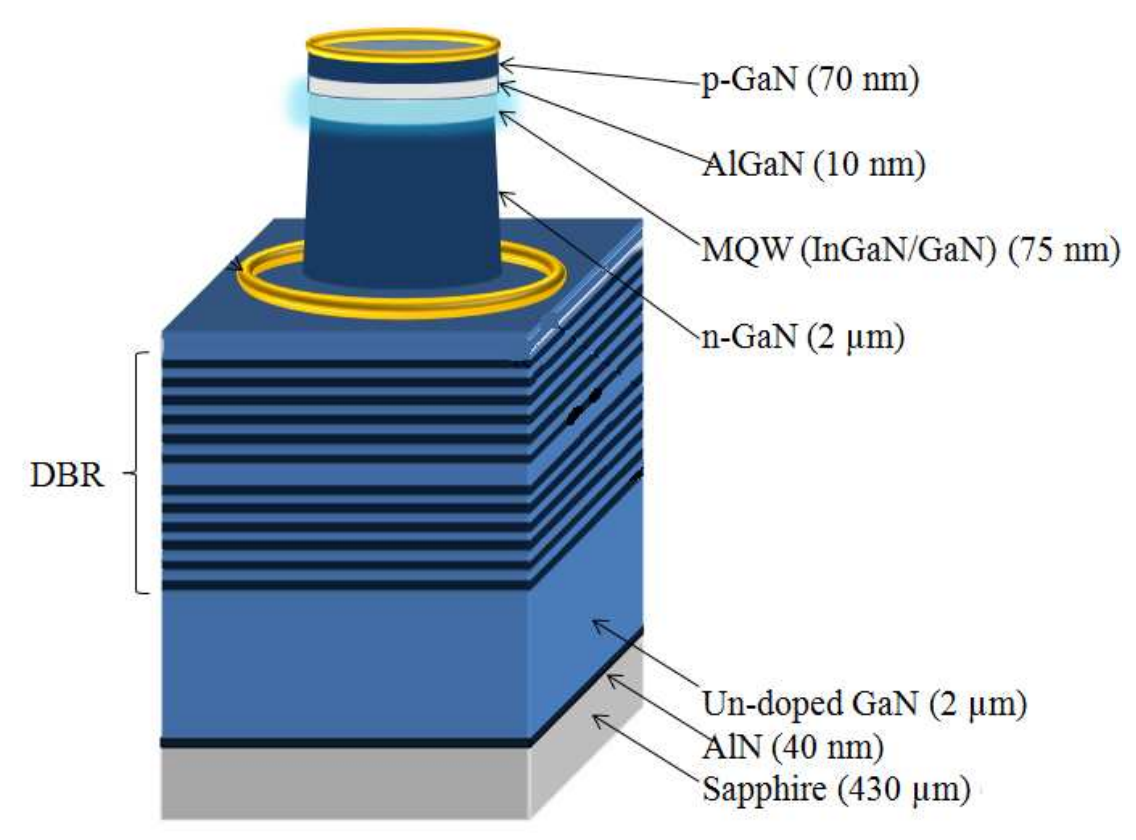

Fig 89 Gallium Nitride LED Structure [84]

The optimum conditions for mode generation and matching optical parameters with those of the experimental values was characterized by changing the refractive indices and thickness of LED layers within an error range of 0.02 refractive index units (RIU) and 10 $\mathrm{nm}$ respectively. This study was used to analyze the effects of various parameters on the behavior of vertical cavity modes (VCMs) and by changing LED parameters to enhance the VCMs for a higher optical emission. Instead of using an external source for coupling light into the LED cavity, rather multiple non-coherent point sources were used as light generation points inside the quantum well region of the LED disk. The VCMs generation due to the internal point sources was characterized and compared with experimental EL. 2$\mathrm{D}$ and 3-D simulation results to help in modeling the thicknesses of layers and to analyze the vertical mode profile, which greatly reduces the power loss into the substrate so the confinement of optical power into the disk can be enhanced. Another structure which can greatly improve light confinement in the LED cavity and reduce substrate losses is the use of a bottom DBR (Distributed Bragg Reflector). It was essential to simulate DBR structures 
before proceeding to LED simulations, and this is discussed in the next section.

\section{3-4-1 DBR Simulations}

$\mathrm{Al}_{x} \mathrm{Ga}_{1-\mathrm{x}} \mathrm{N} / \mathrm{GaN}$ digital alloy DBR structures grown using MOVPE (metal-organicvapor-phase-epitaxy) were used as the bottom LED reflectors [84]. The digital alloy periodicity with alternating GaN and AIN (Aluminum Nitride) layers was simulated for 6 , 12,18 and 25 periods.

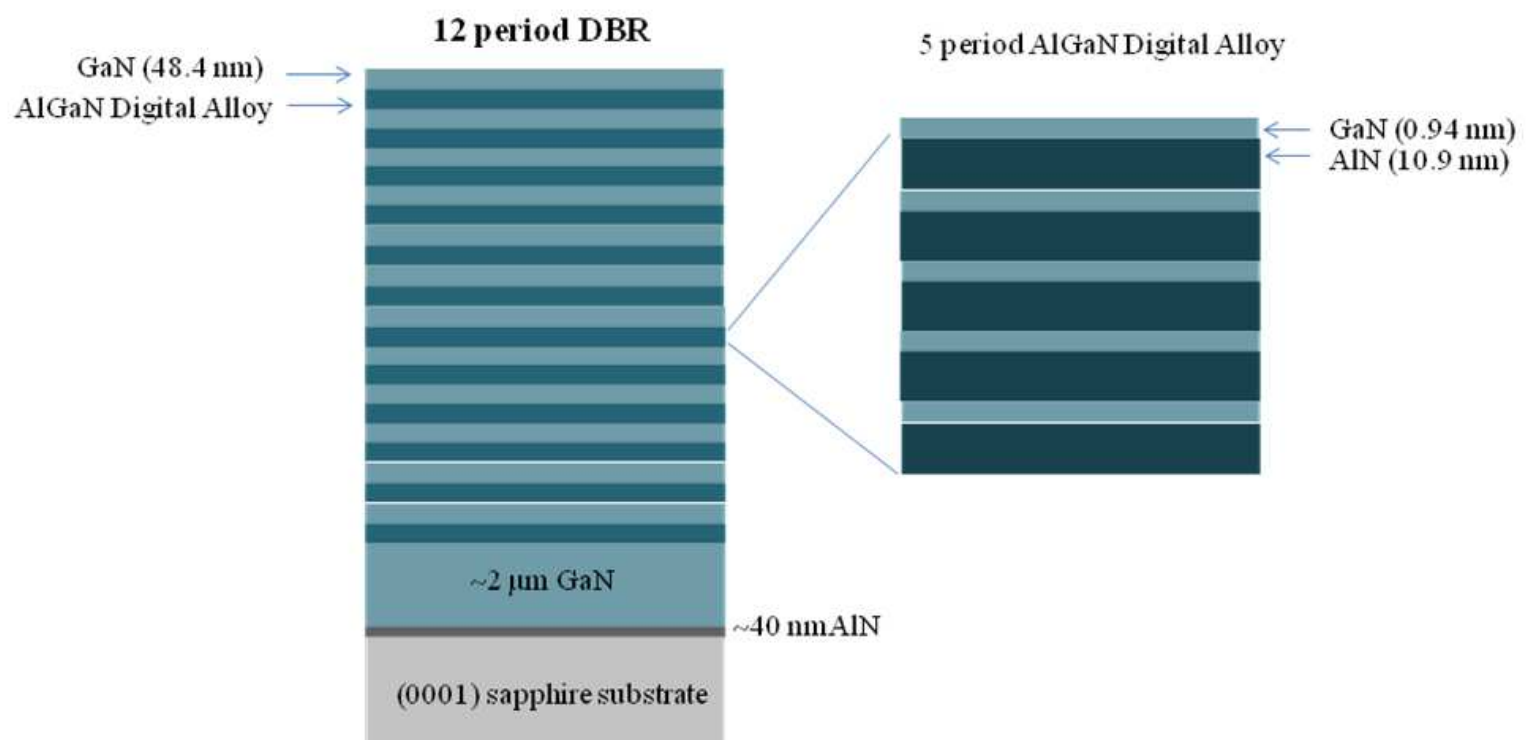

Fig 9012 Period Digital Alloy DBR Structure on Sapphire Substrate [84] 

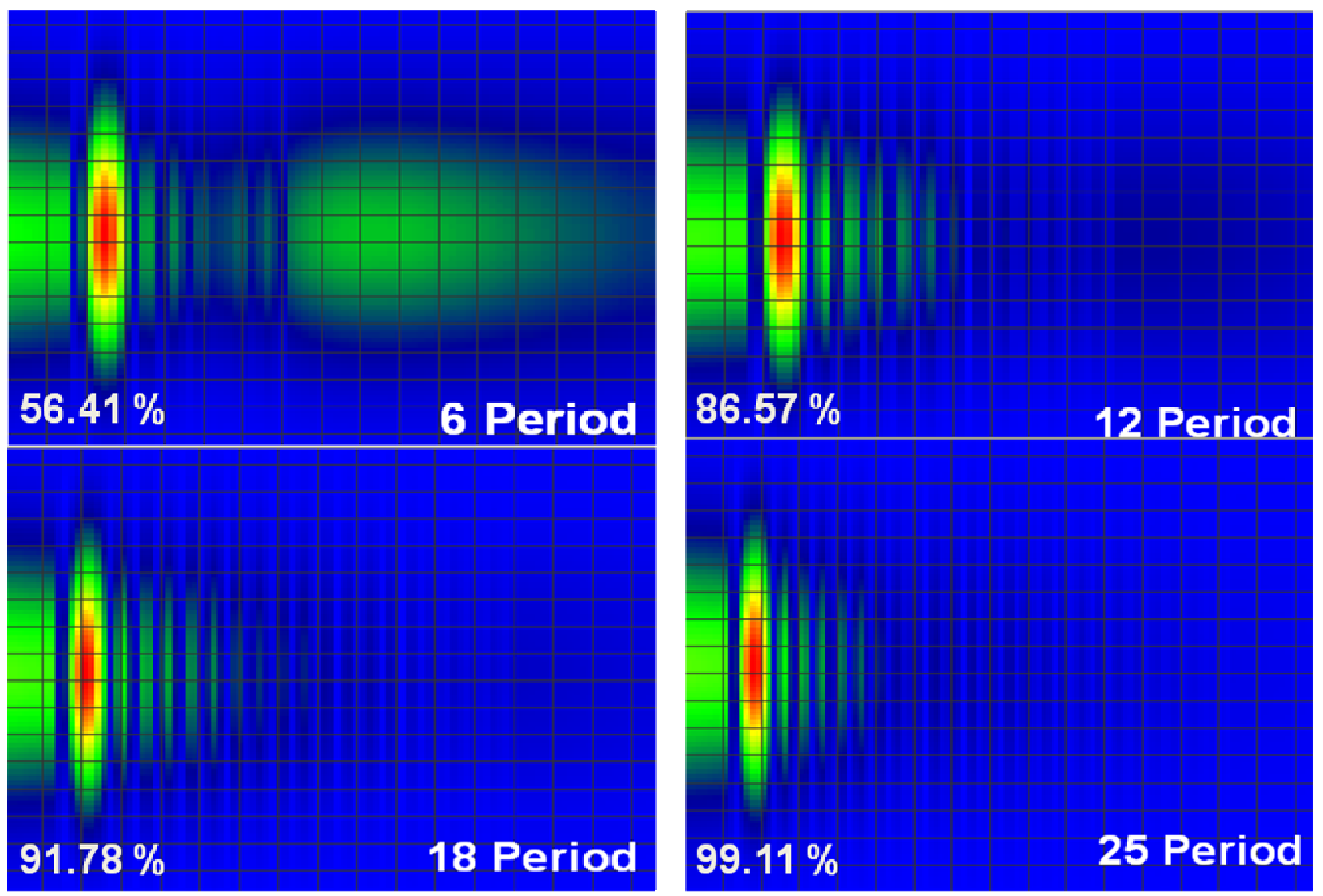

Fig 91 Electromagnetic Intensities for 6, 12, 18 and 25 Period DBR Reflectivity

The DBR reflectivity and bandwidth was characterized and compared with experimental reflectivities. The optical source used was a Gaussian modulated plane wave in space-time with a wavelength range of white light. The input power in the near field ( $500 \mathrm{~nm}$ ) was defined to be more centralized, to couple light along the axis of the DBR layers and to prevent scattering from edges, giving accurate results. A finite computational cell was defined as the simulation volume with the boundary conditions of a perfectly matched layer (PML), to absorb outgoing waves and reduce back-reflections [87]. 

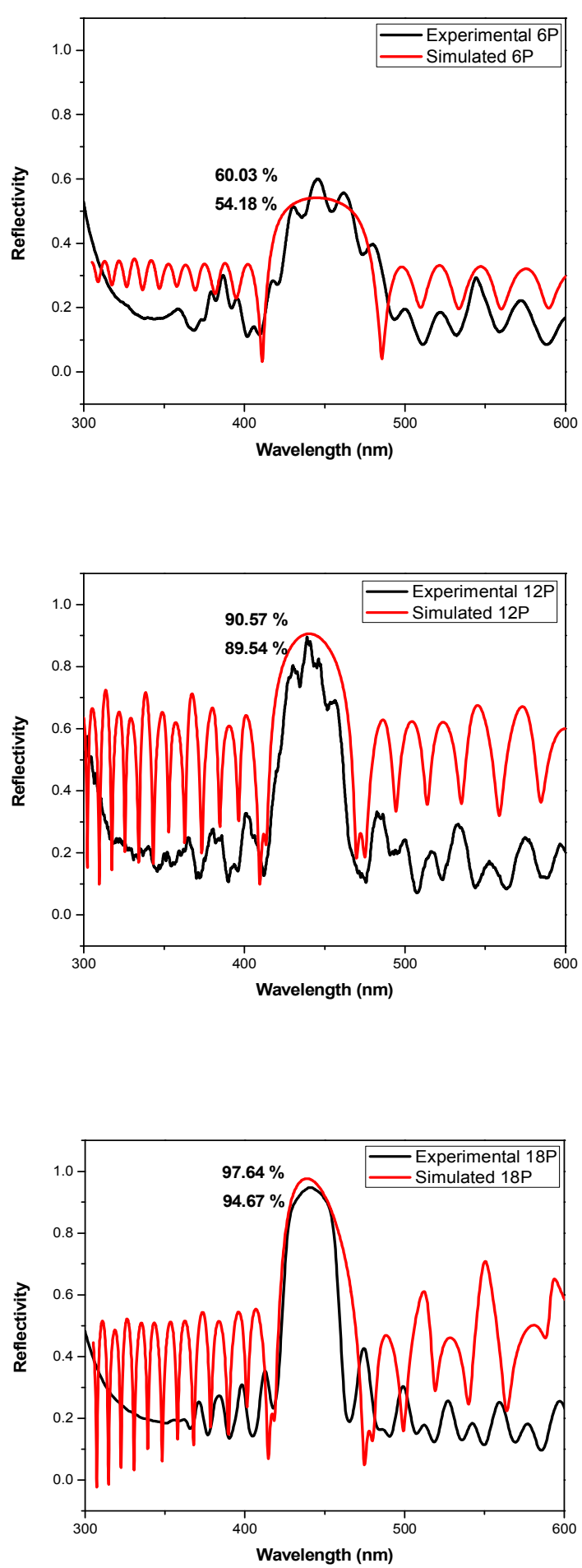

Fig 92 Experimental [88] and Simulated DBR Reflectivities for 6, 12 and 18 Periods 
The simulated DBR reflectivity and bandwidth agreed with the experimental results as shown in the plots.

\section{3-4-2 Passive LED Reflectivity Simulations}

The external excitation (passive) was used to analyze the presence of LED cavity modes before simulating active structures. The reflectivity simulations were performed for RCLEDs on DBR substrates. A plane wave centered at $450 \mathrm{~nm}$ with a FWHM of $100 \mathrm{~nm}$ was introduced at $0.5 \mu \mathrm{m}$ distance from the LED ( $\mathrm{p}-\mathrm{GaN})$ top surface and a reflectivity spectrum was captured. The simulated and experimental reflectivity plots were matched showing the presence of vertical-cavity modes (spectral minima).
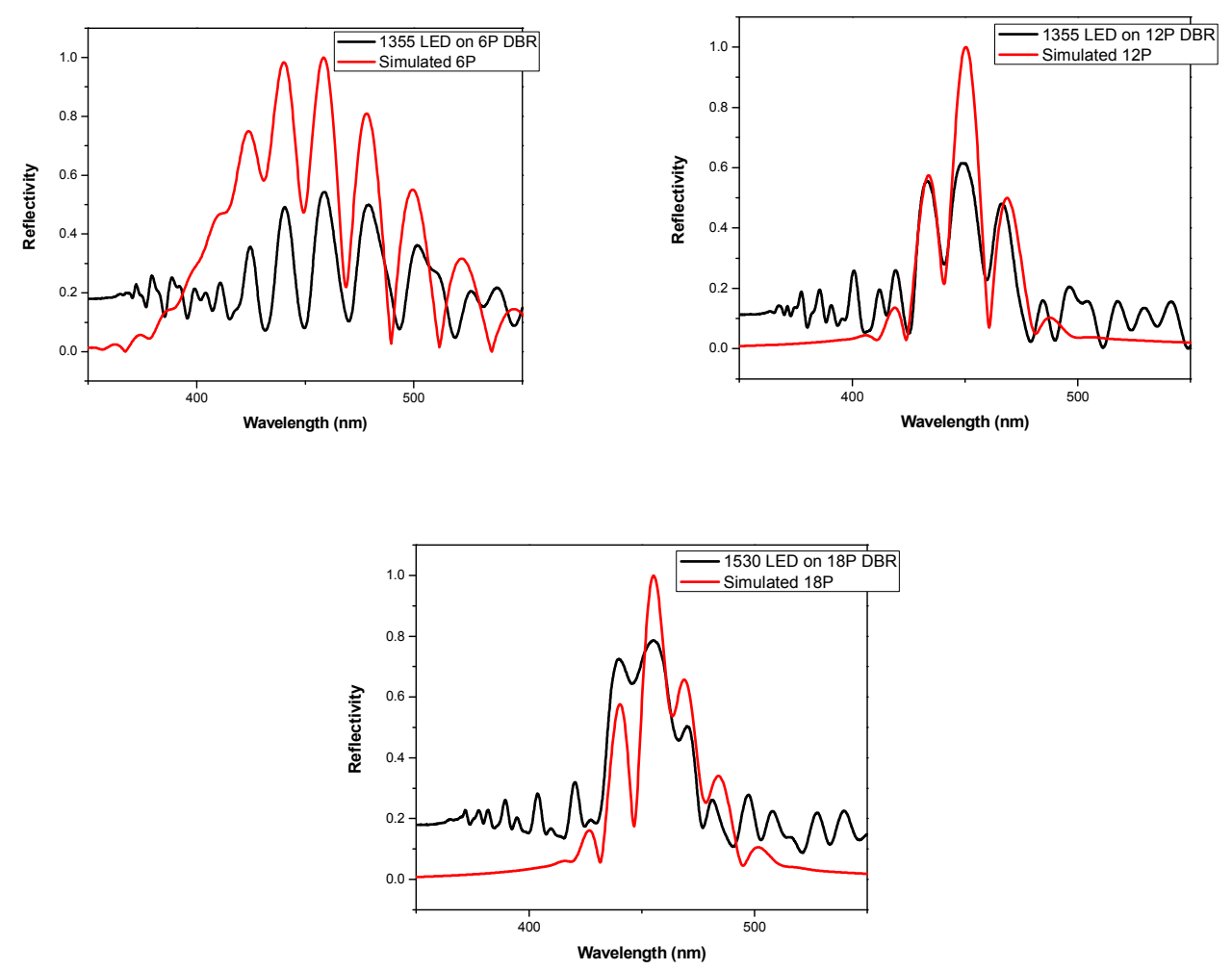

Fig 93 Experimental [88] and Simulated LED Reflectivities on 6, 12 and 18 Period DBR structures 
Analysis indicates that the resonant vertical-cavity modes are formed due to constructive interference of the optical fields in the cavity defined between the LED P-GaN surface (without top mirror) and the buried DBR, and the cavity between the quantum well and DBR. These locations of spectral minima observed in both experimental and simulated spectra are well matched, whereas the spectral intensities differ due to the non-absorptive medium used in the simulation. The simulated spectra were normalized with the maximum input intensity for comparison with the experimental reflectivities. The spectral minimum observed at $432 \pm 2 \mathrm{~nm}$ in LEDs with 6 period DBRs is related to the $8(\lambda / 2)$ resonance of the cavity with a thickness of $1730 \pm 10 \mathrm{~nm}$ between the LED surface and the buried DBR. In the LED with a 12 period DBR, the spectral minimum was observed at $440 \pm 2 \mathrm{~nm}$, which is related to the $7(\lambda / 2)$ resonance of the cavity with a thickness of $1540 \pm 10 \mathrm{~nm}$ defined between the quantum well and the buried DBR.

\section{3-4-3 Vertical Cavity Modes and RCLEDs Simulations}

The vertical cavity modes (VCMs) in RCLED structures were characterized using Opti-FDTD. Mode splitting was analyzed in the disk sidewall electromagnetic profile due to changes in refractive index of LED layers and the substrate. Various LED disk radii and thicknesses were used to characterize vertical cavity mode intensities. From the side view (figure 94) we can observe two distinct vertical cavity modes profiles and one single vertical cavity mode profile depending on the LED thicknesses and refractive indices of the surrounding layers. As the thickness of the disk is increased more vertical cavity modes were observed. 

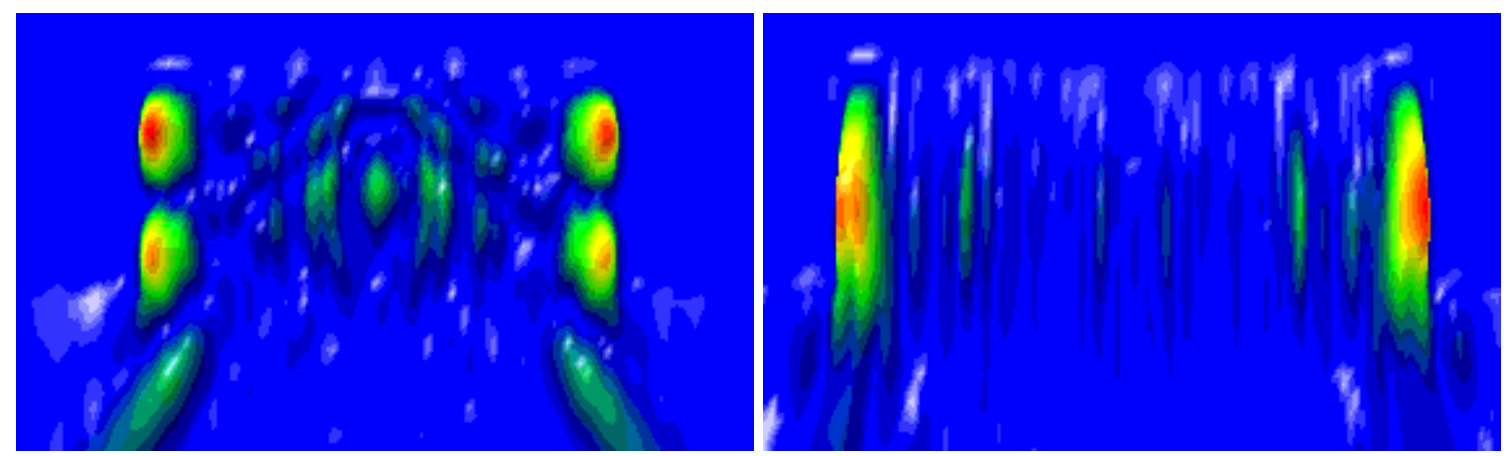

Fig 94 Side View of the Vertical Cavity Modes for a Longer and Shorter LED Cavity

2D simulations can be done to design the mode profile in the disk by choosing appropriate disk and substrate dimensions. Also the power loss into the substrate can be greatly reduced by increasing the disk thickness and shifting the mode profile upwards and away from the substrate.

Therefore the non-uniformity in refractive index on the surface of the disk can greatly change the behavior of vertical cavity modes. Also, increasing the refractive index on top of the disk can shift the mode profile upwards and would reduce the power loss to the substrate. The GaN LED structure was designed with a Distributed Bragg Reflector (DBR) to reduce the substrate power losses and improve the extraction efficiency of the LED. The figure below shows the VCM profile without a DBR.

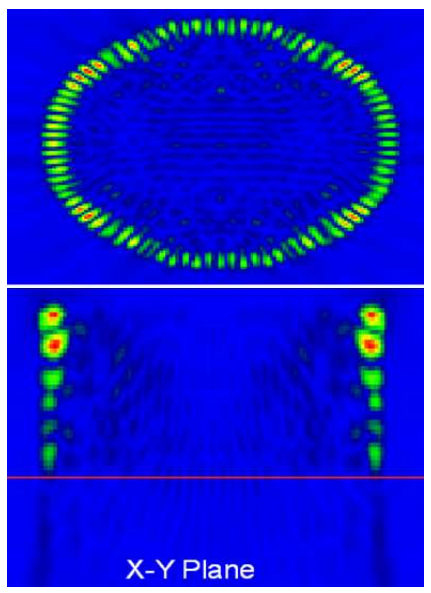

Fig 95 Top (WGM) and Side (VCM) View in LED Cavity (Disk Thickness $=2.15 \mu \mathrm{m})$ 
By simulating the same structure with a DBR the mode profile was shifted upwards and the power loss to the substrate was reduced as shown in the figure below.

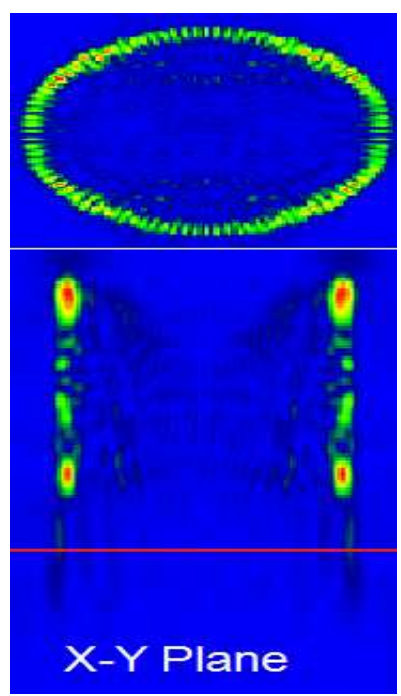

Fig 96 Mode Profile Shift and Reduction in Substrate Power Loss with a DBR LED
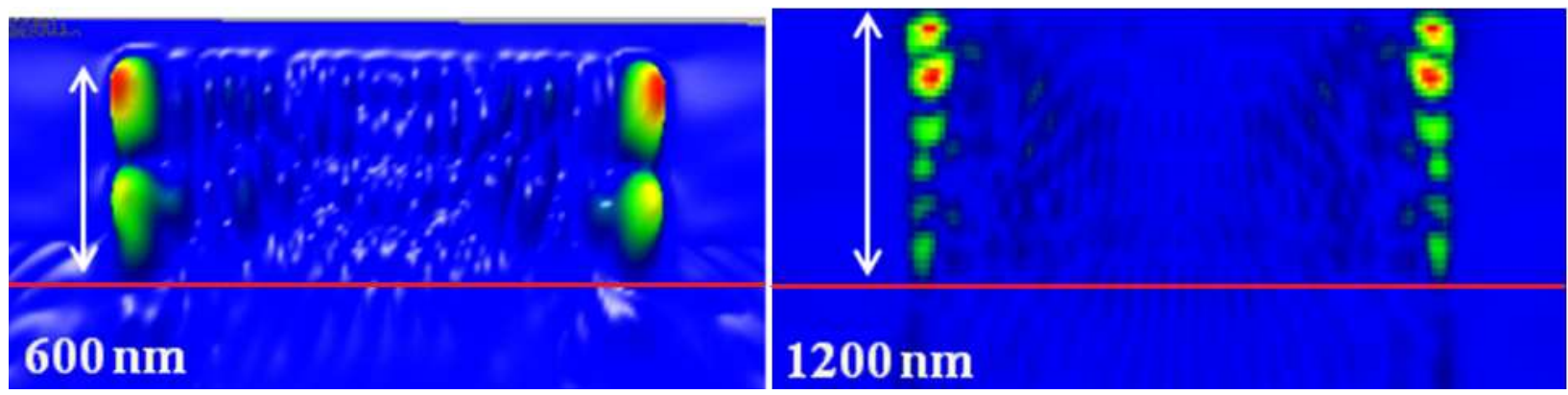

Fig 97 Mode Profile shift and Higher Modes for Longer LED Cavities

Also, the VCMs mode profile can be shifted upwards, reducing substrate loss, by increasing the length of the cavity (few wavelengths) and to enhance the VCM emission from LED top surface. Therefore thickness, indices and position of DBR in RCLEDs play an important role in excitation of optical modes, and properly designed can reduce the substrate losses and enhance emission.

The LED micro-cavity length and positioning of layers with respect to $(\lambda / 2)$ resonance of the cavity lays the basis for design of an RCLED. The cavity acts as a planar 
Fabry-Perot interferometer and the constructive or destructive interference of the fields are a function of the wavelength and cavity length.

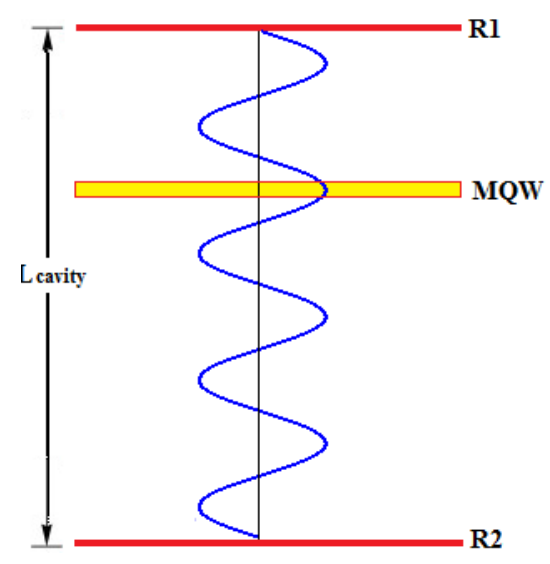

Fig 98 RCLED Cavity Length and Placement of Quantum Well on Anti-node

The cavity length is designed to be a function of $\lambda / 2$ with two reflective surfaces on top and bottom of the cavity. The reflectivity of top surface $\left(R_{1}\right)$ is kept lower than reflectivity of bottom surface $\left(\mathrm{R}_{2}\right)$, for light to emit out from the top. The Multi Quantum Well (MQW) is placed at an anti-node of the standing wave to constructively interfere the wave inside the designed cavity. The boundaries of the LED layers are kept at the nodes to reduce internal reflections and improve wave propagation. The optical intensity of the RCLED at a wavelength of $450 \mathrm{~nm}$ was simulated as a function of cavity length using MATLAB. 


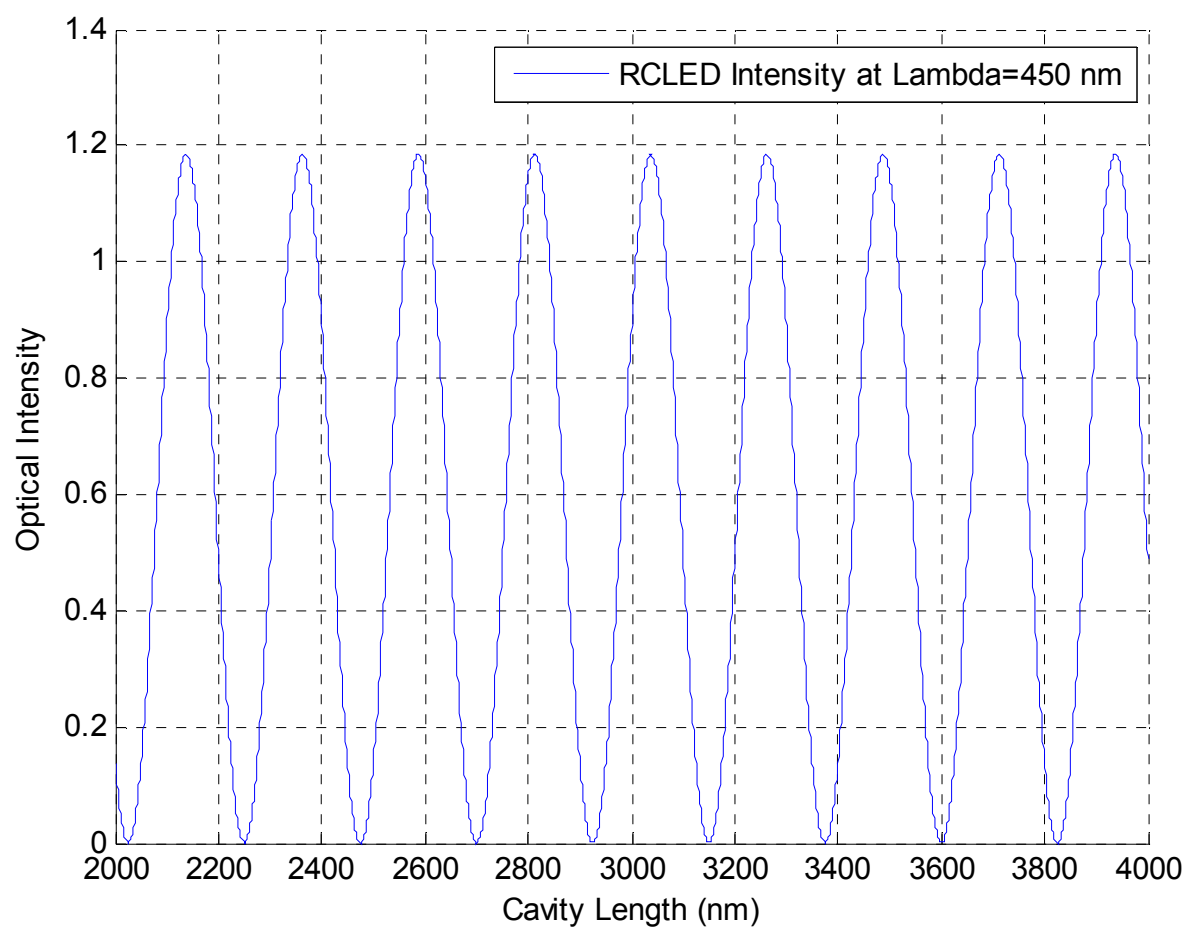

Fig 99 RCLED Optical Intensity as a Function of Cavity Length

As seen in the MATLAB plot, the optical intensity outside the LED drops to zero at resonant wavelengths as those wavelengths constructively interfere inside the cavity. Due to higher absorption losses or lower Q factors of the cavity, the intensity of resonant modes is weaker outside the cavity. As the Q-factors increase the strongly coupled resonant cavity modes can be extracted outside the cavity with an increased LED emission and a narrower FWHM. Opti-FDTD simulations were used to analyze this effect. A planar LED structure on a DBR substrate with non-resonant cavity thicknesses (as experimentally used) was simulated and compared to a designed RCLED structure. 
LED

\begin{tabular}{|l|l|l|l|l|}
2.45 & 2.4 & 2.48 & 2.45 & \\
p-GaN & $\begin{array}{c}\text { P-Al } \\
\text { GaN }\end{array}$ & MQW & $n-G a N$ & $90 \%$ DBR \\
\hline
\end{tabular}

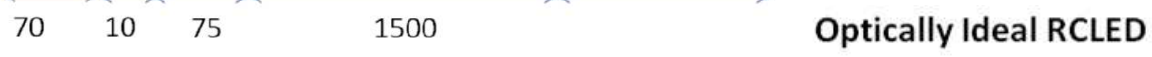

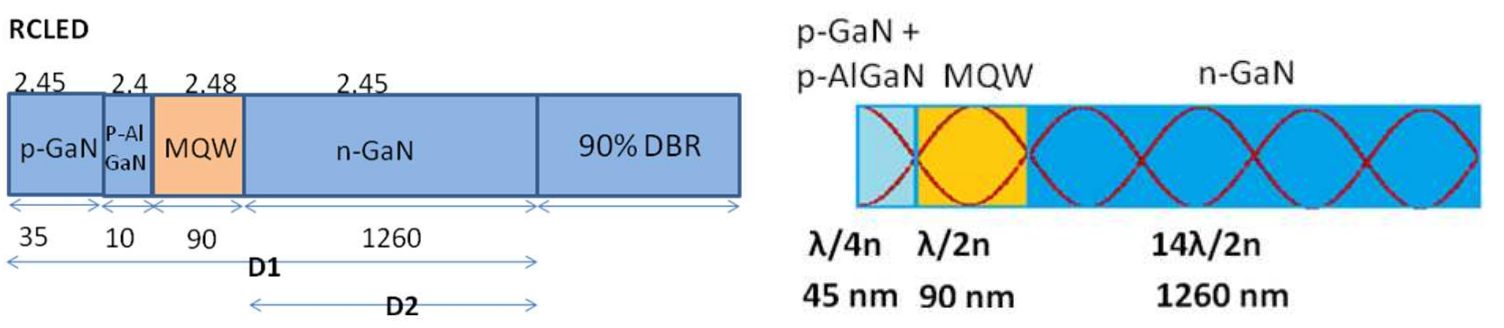

Fig 100 LED and Designed RCLED Structures (Showing Refractive Indices) and Optical Cavities D1, D2

The simulation shows an enhanced output emission intensity of the RCLED structure, and an improvement of $110 \%$ was observed for a peak wavelength of $441 \mathrm{~nm}$ and FWHM of $25 \mathrm{~nm}$.
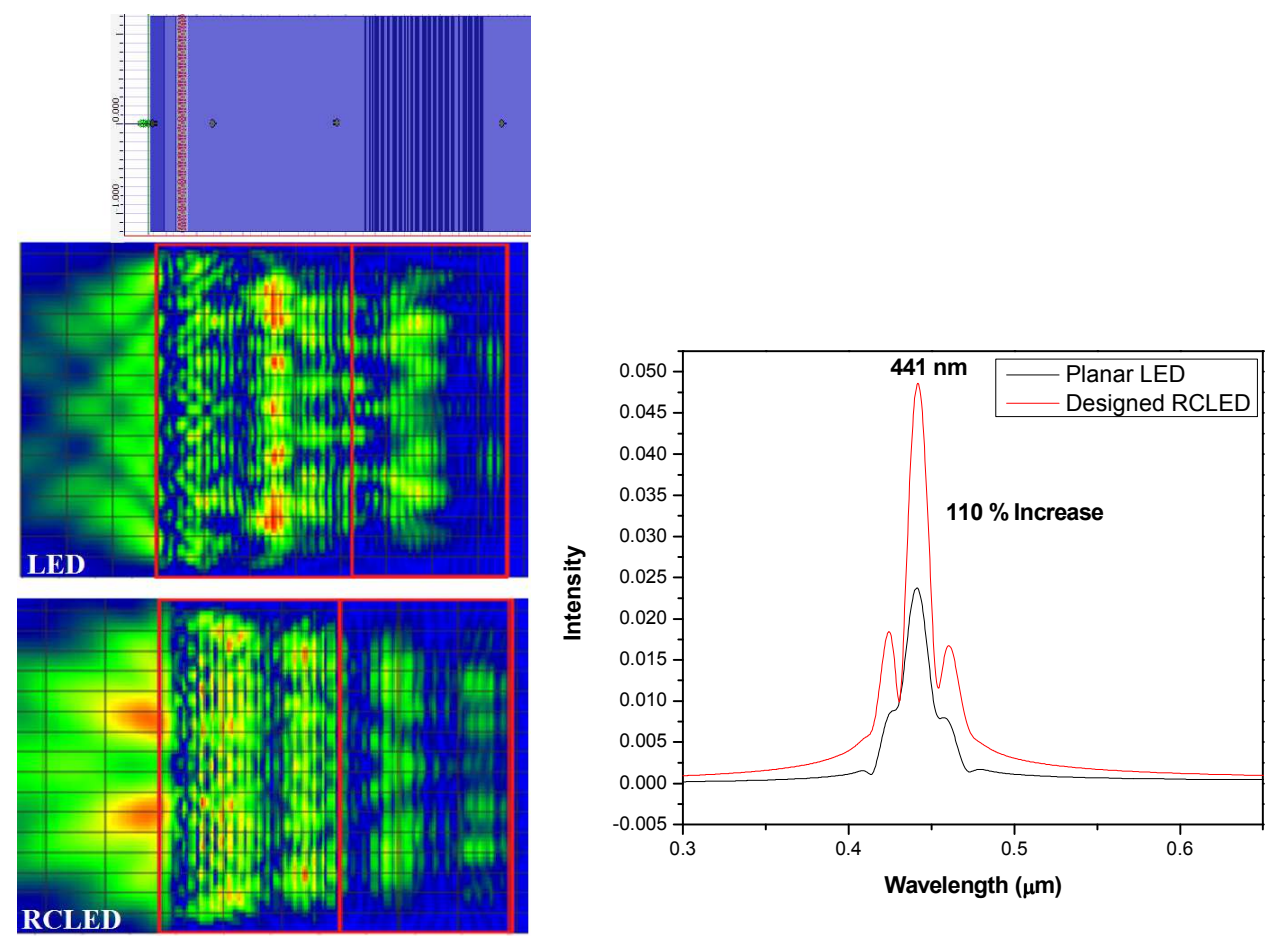

Fig 101 Simulated Intensity (Left) and Spectra (Right) for LED and Designed RCLED 
The optical emission was captured using observation points and planes from a distance of $500 \mathrm{~nm}$ above the LED P-GaN surface, and showed an improvement in the emission intensity. The spectral minima observed in the simulated LED EL spectrum were due to vertical cavity modes in the LED cavity. Next, LED EL spectra were simulated, analyzed for VCMs and matched with experimental emission.

\section{3-4-4 LED Electroluminescence Simulations}

The finite difference time domain (FDTD) method was used for active (Internally excited) LED EL simulations. The FDTD method is different from other modeling techniques such as the FEM (Finite Element Method), where a steady state solution is used as compared to the time-domain method of FDTD. The time-varying fields introduced into the simulation space propagate and interact with LED layers and the interference effects can be modeled, making it a useful tool for analysis of resonant cavity modes in LED devices. For Resonant Cavity (RC) LEDs, the quantum well emitting layer is surrounded by several optically thin layers, producing interference effects and also trapping light into the device due to high refractive indices of the layers. Understanding of the electromagnetic field distributions in the LED layers can not only improve the extraction efficiency but also reduce the optical losses present in the LEDs due to vertical cavity modes. The optical power in different layers of a DBR LED disk structure can be seen in the figure below. Ring n-metal and p-metal contacts were used to generate a uniform refractive index profile of the disk. 

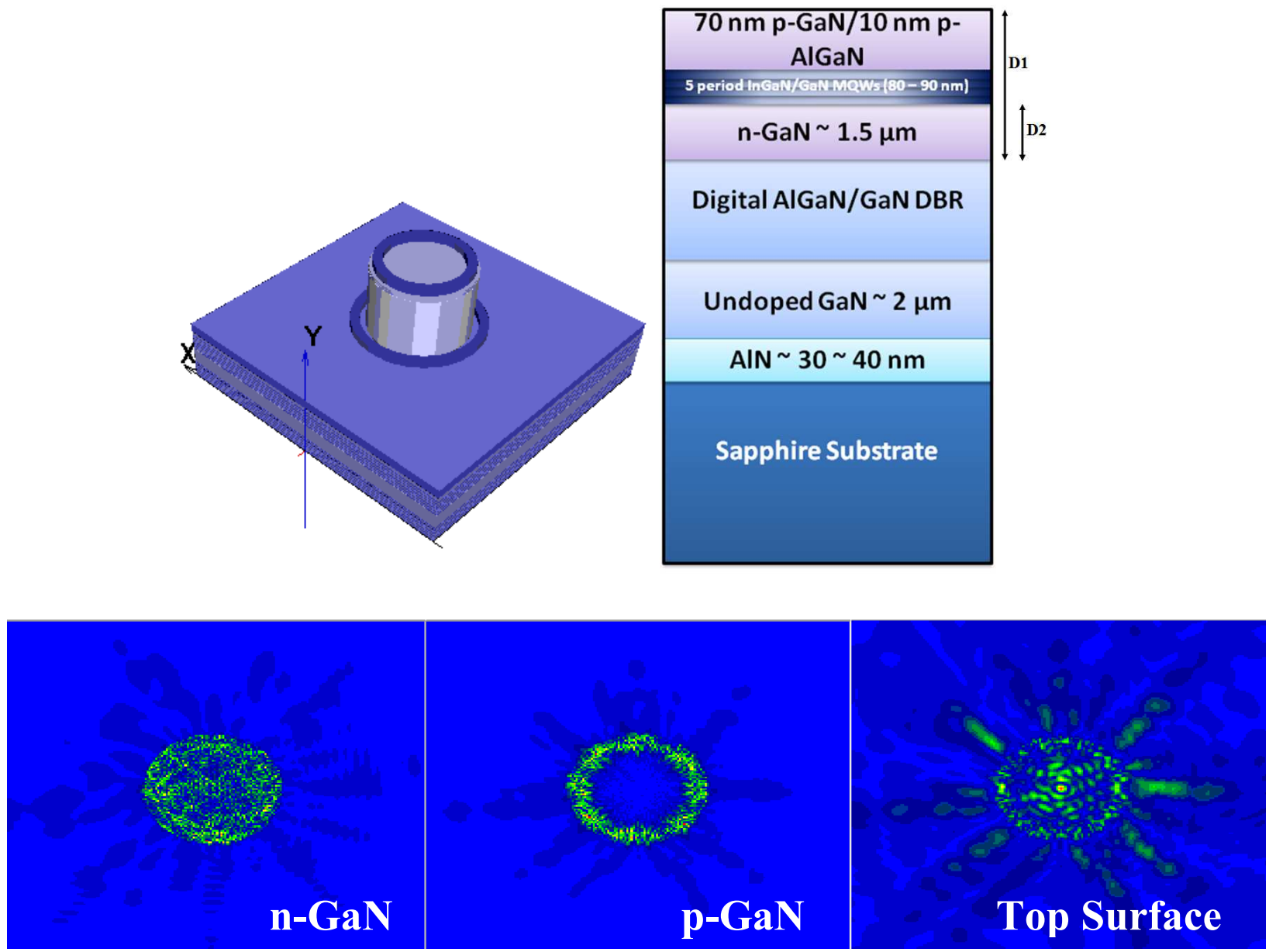

Fig 102 Simulated LED Structure and Optical Intensities in Different Regions of LED

To model these LED structures, non-coherent Gaussian modulated continuous spherical wave (GMCW) point sources were embedded into the quantum well region to simulate the optical emission from the active region of the LED. Multiple spherical wave point sources represent more localized quantum well emission as compared to a plane wave. 


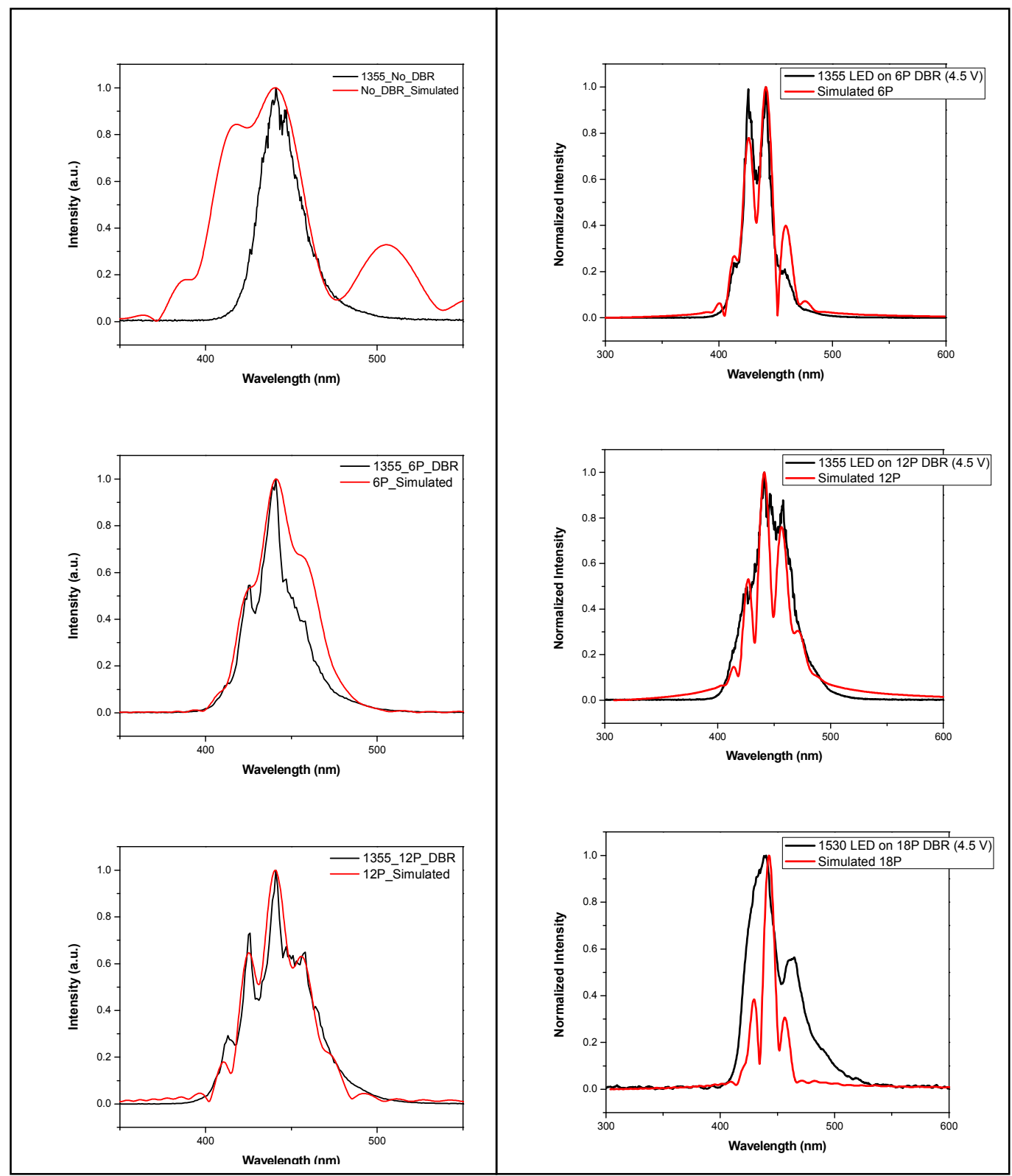

Fig 103 Simulated and Experimental [88] LED EL Plots at 4V (Left) and 4.5 V (Right) for No DBR, 6, 12 and 18 Period DBR LEDs

A simulation region size of $5 \times 10 \mu \mathrm{m}^{2}$ was uniformly discretized with a mesh size of $0.03 \mu \mathrm{m}$ and perfectly matched layer (PML) boundary conditions were used at the simulation region boundaries to absorb outgoing waves and reduce back-reflections [87]. 
The thicknesses and indices of the LED layers were designed and matched to experimental values within an error of $\pm 10 \mathrm{~nm}$ and \pm 0.02 RIU (Refractive Index Units). Observation points on the top and bottom of the LED structure were used to collect the spectral emission data. The resonant vertical cavity modes (spectral minima) observed in the simulated spectra matched well to the experimental EL spectra. The resonant dominant modes are approximately observed in both the experimental and simulated spectra at $432 \pm 2 \mathrm{~nm}$ and $451 \pm 2 \mathrm{~nm}$, corresponding to the cavity defined between the LED surface and the buried DBR $(1730 \pm 10 \mathrm{~nm})$, and between the quantum well and DBR $(1575 \pm 10 \mathrm{~nm})$, respectively. The vertical-cavity modes are an adequate representation of the field patterns formed due to constructive interference in the cavity and resonance at certain wavelengths, as seen in the output spectrum. In order to reduce the optical losses experienced by the vertical cavity modes, and to extract the trapped modes by improving the quality factors of the cavity, and designing the thicknesses/Indices of layers, we optimized the simulation of the RCLED structure using the FDTD method and then confirm the presence of resonant vertical cavity modes by also performing reflectivity measurements on DBR embedded LED structures. 


\section{Chapter 4}

\section{Summary and Conclusions}

Optoelectronic micro-resonators form a basis for the next generation compact, high speed and low power photonic circuits. Designing the shape, size and materials of the resonator can be tuned to enhance the MDRs (Morphology Dependent Resonances) with required polarization, bandwidth, frequency and emission spectrum. This opens doors to potentially develop new types of photonic devices like LEDs, lasers, optical switches, filters etc. Novel designs create a scope of challenging fundamental physics applications beyond current technologies. Fabry perot based planar optical resonators are utilized in every field of linear and nonlinear optics. When high performance and sensitivity of devices is required, micro-cavity morphology-dependent circular resonators based on total internal reflection become an alternative to their planar traditional counterparts. Photonic circuits utilizing strong optical confinement in planar waveguides, micro-disks, and micro-rings are promising candidates for large scale integration, and very sensitive to fabrication errors which can drastically effect the coupling efficiency. Accurate fabrication of optical coupling gaps $(<0.1 \mu \mathrm{m})$ by photo/e-beam lithography and etching is still a challenging task. In this dissertation we have reviewed the state of the art optoelectronic resonator tools, fabrication and characterization techniques and the challenges for potential of bio-sensing and enhanced emission intensities.

The first sensitivity and selectivity characterization of a resonant planar alumina waveguide stack was achieved, and the device performance with refractive index changes of surface loading, and also with the fabricated waveguide parameters was analyzed. The effective surface loading detection sensitivity was experimentally determined to be 20 $\mathrm{pg} / \mathrm{mm}^{2}$ with a bulk index sensitivity of $5.6 \times 10^{-4}$ Refractive Index Units (RIU) for aqueous sucrose solutions. Circular cavity MDRs based devices such as microspheres and microdisks were studied, which enhances and distributes the optical resonant frequencies as 
compared to a planar macro-cavity. The unique modal properties of passive WGMs (Whispering Gallery Modes) were researched and detected in micro-spheres/disks, including linewidth, tunability, bandwidth, Q-factors and peak shifts due to refractive index changes. Photonic sensors and filters are amongst the most developed devices exploiting WGMs. Simulations of planar waveguide sensor coupled with polystyrene microspheres showed the presence of WGMs, which was the main cause of scattering in microspheres and power losses in the waveguide. Surface coverage of microspheres on an alumina waveguide were experimentally detected to have a minimum surface coverage limit of $0.192 \%$ based on the sensitivity to waveguide output power.

Passive WGMs in GaN micro-disks were shown both in simulations and experimentally. A variation in mode spacing of $3 \mathrm{~nm}$ to $7 \mathrm{~nm}\left(\lambda^{2} / 2 \pi \mathrm{Rn}\right)$ was tuned in as the disk radius was reduced from $4.5 \mu \mathrm{m}$ to $2 \mu \mathrm{m}$. The disk index, thickness and material parameters can be used for sensing applications and whispering gallery resonator spectroscopy can be utilized to enhance interaction between photons and atoms/molecules. WGMs based biosensors have been reported in literature and typically detect presence of refractive index changes due to molecular binding producing spectral shift of the order of picometers [89]. The main advantages for this technique are improved selectivity, sensitivity, non-destructive and applicability to various samples. However, most WGM biosensors rely on measurements related to transmission spectra or scattering characteristics of an optical mode in the presence of a biomolecule. The sensors can also measure the change in phase or intensity of light at the waveguide output. Achieving high Q-factors of micro-resonators is a challenge and most crucial for sensitivity of the device. Fabrication challenges for improved Q factors have scope for future work, as lithography resolution and etching smooth sidewalls with lower surface scattering can be improved.

Another major application of micro-resonators is in novel generation of light sources such as Resonant Cavity (RC) LEDs. The active generation of vertical cavity modes in electrically pumped LEDs was characterized using simulations and experimentally. 
Wavelength-sized micro-disk LED structures were designed in which the electromagnetic fields can either be destructively suppressed or enhanced, with a narrowing of spectral linewidth and improvement in the spectral intensity. It was observed that the spontaneous emission from a MQW (Multi Quantum Well) can be significantly altered when it was placed at different locations in a high-loss cavity. This gives scope to explore various new micro-disk/ring designs for efficient control of spontaneous emission at the LED output. Proper design and placement of LED layers supports high-Q vertical cavity modes, and emission at one modal wavelength can be strongly enhanced. The main challenge is to develop cavity-enhanced LEDs with high-Q modes, which is not easily achieved in a bulk or hetero-structure MQW LEDs. Semiconductor Quantum Dots (QDs) are a recently proposed concept which can be explored in future work, to enhance cavity gain. The QDs can be fabricated using self assembly techniques and integrated as atomic emitters into the LED disk. The next challenge is to efficiently design micro-resonator geometrical properties to manipulate and increase the strength of coupling between QD and optical fields of the resonator. Alternatively, micro-resonators can be designed with non-symmetric (elliptical) geometries to obtain directional emission patterns with applications in lasing. In this work, the micro-disk geometries were considered for active generation of Vertical Cavity Modes (VCMs). Micro-cylindrical DBR RCLEDs greatly enhanced the VCMs and output emission with properly designed layer thicknesses and MQW (multi quantum well) placement in the cavity. Both experimental and simulated LED spectra confirm the presence of VCMs with matched spectral minima at $432 \mathrm{~nm}$ and $451 \mathrm{~nm}$ confirming VCMs related to $(\lambda / 2)$ cavity resonances.

Advancements in the micro/nano-fabrication technologies offer great potential to fabricate novel optical resonator structures with improved control in material systems. Future applications include nano-scale resonators for optical signal processing in low cost, high speed, large scale integration photonic circuits. Another area of interest is the enhancement of detection sensitivity for biosensors, with more compact and efficient optical structures with enhanced functionality. Continued development of a new range of materials such as quantum wires or nanotubes with special designed properties is expected to bring 
more high performance optical resonators. The emerging all-optical resonator designs demands strict requirements in accuracy, performance to develop advanced devices and structures. 


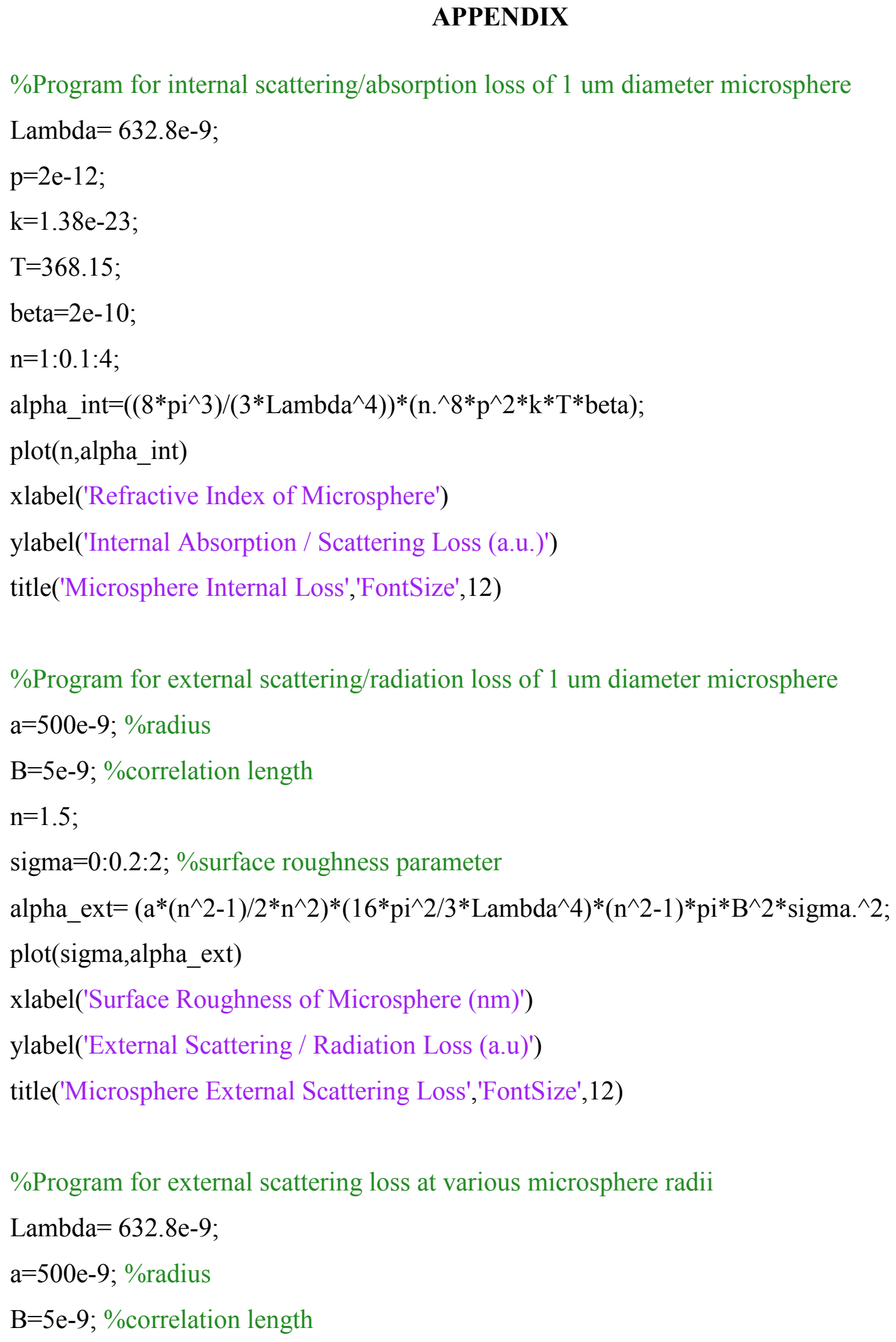




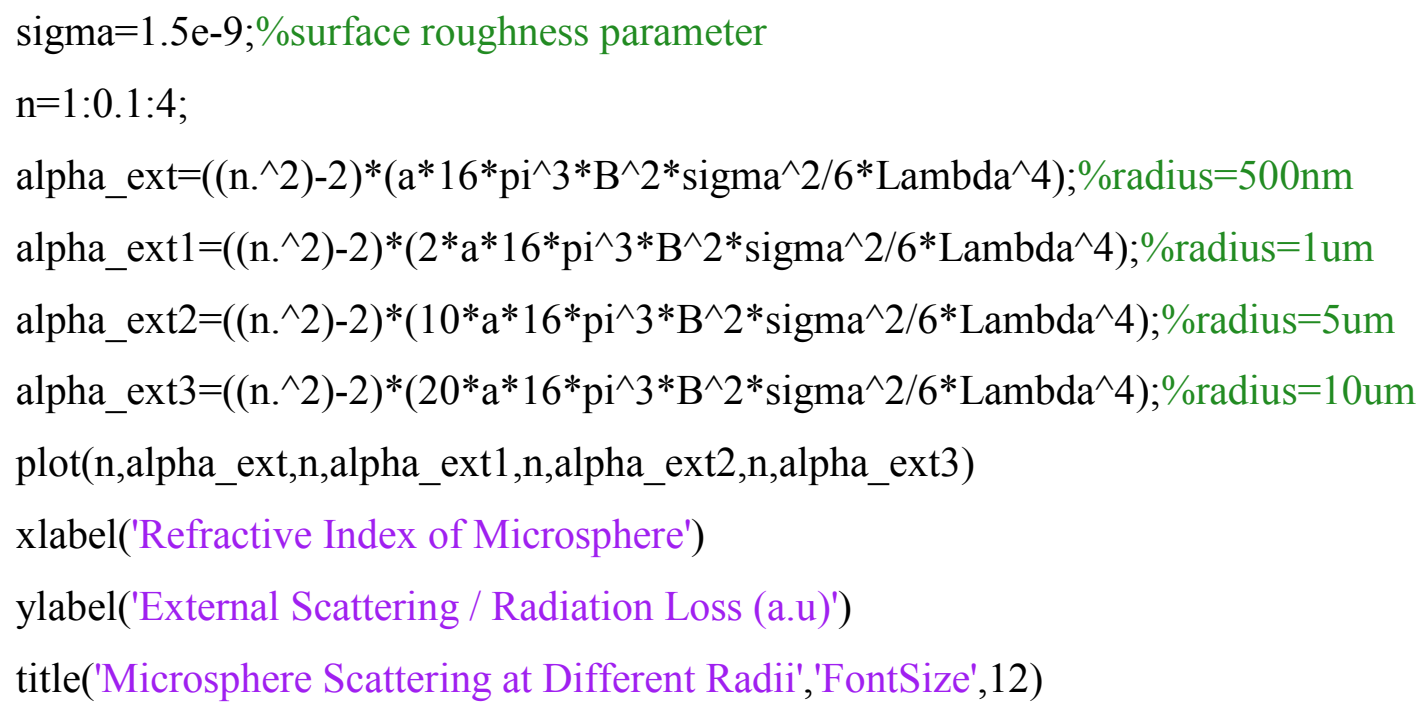




\section{REFERENCES}

1. R. Narayanaswamy, O.S. Wolfbeis, Optical Sensors, Springer, New York, 2004.

2. I.M. White, X. Fan, Opt. Express 16 (2008) 1020.

3. N.A. Mortensen, S. Xiao, J. Pedersen, Microfluid. Nanofluid.4 (2008) 117.

4. L. Rindorf, O. Bang, Opt. Lett. 33 (2008) 563.

5. Smith, L. M.; Saunders, J. Z.; Kaiser, R. J.; Hughes, P.; Dodd, C. R.; Cornell, C. R.; Heiner, C.; Kent, S. B. H.; Hood, L. E. "Fluorescence Detection in Automated DNA Sequence Analysis", Nature, 1986, 321, 674-679.

6. http://web.uvic.ca/ail/techniques/epi-fluorescence.html

7. G. Herzberg, "Infrared and Raman Spectra of Polyatomic Molecules", Van Nostrand Reinhold, New York, NY, 1945.

8. J. C. de Paula, http://www.haverford.edu/chem/302/Raman.pdf.

9. Paddle, B.M. 1996. Biosensors for chemical and biological agents of defense interest. Biosens. Bioelectron. 11 (11), 1079

10. http://en.wikipedia.org/wiki/Surface_plasmon_resonance.

11. Exploitation of Localized Surface Plasmon Resonance, Eliza Hutter, Janos Fendler, Advanced Materials, 16, No. 19, 2004.

12. Localized Surface Plasmon Resonance Biosensors, J. Zhao, X. Zhang, C. R. Yonzon, A. J. Haes, R. P. Van Duyne, Future Medicine, 2006.

13. Localized Surface Plasmon Resonance Spectroscopy and Sensing, Katherine A.Willets and Richard P. Van Duyne, Annu. Rev. Phys. Chem. 2007. 58:267-97.

14. "Guided-Wave Optical Biosensors", Vittorio M. N. Passaro, Francesco Dell'Olio, Biagio Casamassima and Francesco De Leonardis, Sensors, 25 April 2007,7, 508536.

15. "Resonant Detection of Nano to Microscopic Objects Using Whispering Gallery Modes", PhD Dissertation, Frank Vollmer, The Rockefeller University, 2004.

16. "Application of Ion Beam Assisted Thin Film Deposition Techniques to the Fabrication of a Biosensor Chip with Fieldability Potential for Important Biohazard Detection Applications", D. Lloyd, L Hornak, S Pathak, D Morton, I. Stevenson, 
47th Annual Technical Conference Proc., Soc. Of Vac. Coaters, ISSN 0737-5921 (2004).

17. P. Poloju, P. Samudrala, J. R. Nightingale, D. Korakakis, L. A. Hornak, "Characterization of Alumina Optical Waveguides Grown by Ion Beam Assisted Deposition for SPARROW Biosensors," MRS Fall 2006 Conference Proceedings.

18. S. Nakamura, "InGaN-based blue laser diodes," Selected Topics in Quantum Electronics, IEEE Journal of, vol. 3, pp. 712-718, 1997.

19. V. M. Bove and W. Sierra, "Personal Projection," SMPTE Imaging, pp. 17-21, 2004.

20. J. Piprek, Semiconductor Optoelectronic Devices: Academic Press, 2003.

21. Braun, G. Ihlein, J. U. Nokel, G. Shulz-Ekloff, F. Shuth, U. Vietze, O. Weib, and D. Wohrle, "Hexagonal microlasers based on organic dyes in nanoporous crystals," Applied Physics B., vol. 70, pp. 335-400, 2000.

22. T. Nobis, E. M. Kaidashev, A. Rahm, M. Lorez, and M. Grundmann, "Whispering gallery modes in nanosized dielectric resonators with hexagonal cross section," Physical Review Letters, vol. 93, pp. 1039031-1039037, 2004.

23. J. Wiersig, "Hexagonal dielectric resonators and microcrystal lasers," Physical Review A (Atomic, Molecular, and Optical Physics), vol. 67, p. 023807, 2003.

24. J. Wiersig, "Boundary element method for resonances in dielectric microcavities," Journal of Optics A: Pure and Applied Optics, vol. 5, pp. 53-60, 2003.

25. J. Wiersig, "Hexagonal dielectric resonators and microcrystal lasers," Physical Review A, vol. 67, p. 023807, 2003.

26. R. E. Slusher, A. F. J. Levi, U. Mohideen, S. L. McCall, S. J. Pearton, and R. A. Logan, "Threshold characteristics of semiconductor microdisk lasers," Applied Physics Letters, vol. 63, pp. 1310-1312, 1993/09/06/ 1993.

27. S. Ramo, J. R. Whinnery, and T. V. Duzer, Fields and waves in communication electronics, 3rd ed.: John Wiley \& Sons, 1993.

28. S. Simhony, E. M. Kosower, and A. Katzir, "Novel attenuated total internal reflectance spectroscopic cell using infrared fibers for aqueous solutions," Appl. 
Phys. Lett. 49, 253-254 (1986).

29. P. H. Paul and G. Kychakoff, "Fiber-optic evanescent field absorption sensor,'AAppl. Phys. Lett. 51, 12-14 (1987).

30. S. Simhony, I. Schnitzer, A. Katzir, and E. M. Kosower, "Evanescent wave infrared spectroscopy of liquids using silver halide optical fibers,' J. Appl. Phys. 64, 37323734(1988).

31. Chen H.C, 1985 - Theory of Electromagnetic waves. Mc Grow - Hill Book Company Inc., International Student Edition, Singapore, Page: 58.

32. Cheng D.K., 1989 - Field and wave. Addison Wesley publishing Company, $2^{\text {nd }}$ Edition, United States of America, Page: 323.

33. Qeshta M.N., and Al-Juaidi A.A., 2009 - Optical wave guide Sensor P: 1.

34. Sadiku M.N.O., 2001 - elements of electromagnetics, Oxford University Press, Inc, $3^{\text {rd }}$ Edition, New York, P: 389.

35. Lee, Donald L., Electromagnetic Principles of Integrated Optics, John Wiley \& Sons; (1986)

36. Prillor F., Gilles H., Girard S., larche M., Kaiser r., and Gazibegovic A., 2005 Goos - Hänchen and Imbert - Fedorov Shifts for leaky guided modes. J. Opt. Soc. Am., Am., Vol. 22, No. 6, Page: 1290.

37. D. Marcuse, Bell Syst. Tech. J. 48 (1969) 3187.

38. Palais, J.C., Fiber Optic Communications. Prentice Hall, April, 2002

39. Liang, A.H., The Rayleigh Scattering Loss of Isotropic Weakly Guiding Single-Mode Waveguides. IEEE Journal of Quantum Electronics. V28, n9, p1844-1847, 1992.

40. F. P. Payne, J. P. R. Lacey, "A theoretical analysis of scattering loss from planar optical waveguides", Optical and Quantum Electronics, 26 (1994) 977-986.

41. Ke Feng, Biolayer modeling and optimization for the SPARROW biosensor, PhD Dissertation, West Virginia University, 2007.

42. Ogilvy, J. A., Rough surfaces: Gaussian or exponential statistics? J. Phys. D: Appl. 
Phys 22, p1243-1251, 1989.

43. Ladouceur, F., Roughness, Inhomogeneity, and Integrated Optics. Journal of Lightwave Technology. V15, n6, p1020-1025, 1997

44. M. Kuznetsov, H. A. Haus, Radiation loss in dielectric waveguide structures by the volume current method, Oct 1983, Vol. QE-19, No. 10, IEEE Journal of Quantum Electronics.

45. Jin, G.H., Harari, J., Joannes, L., Vilcot, J.P., Decoster, D., Numerical analysis of the radiation losses due to surface roughness in integrated optics devices. IEEE photonic tech. letters, V8, n9, p1202-1204, 1996

46. J J Gerdes. Bidirectional eigenmode propagation analysis of optical waveguides based on method of lines. Electronics Letters, 30(7) 550, March 1994.

47. S. T. Peng and T. Tamir. TM mode perturbation analysis of dielectric gratings. Applied Physics, 7(35) 35-38, 1975.

48. Jose Rodriguez, R. D. Crespo, and S. Fernandez. Radiation losses on discontinuities in integrated optical waveguides. Optical Engineering, 38(11) 1896-1906, November 1999.

49. Al-Bader S. J. and Jamid H. A. Mode scattering by a non-linear step-discontinuity in dielectric optical waveguides. IEEE Transactions on Microwave theory and Techniques, 44(2) 218-224, February 1996.

50. P. Poloju, "Fabrication and Functional Analysis of SPARROW Biosensor," Master's thesis, Lane Dept. Elec. Eng., West Virginia University, Morgantown, WV, 2007.

51. Mehjez E.M., 2008 - Metamaterial optical wave guide sensor. Dar El-Arqum, P:10

52. Parriaux O. and Velduis G.J., 1998 - Normalized analysis for the sensitivity optimization of integrated optics evanescent - wave sensors. J. of light Tech., 16,4,p : $573-582$.

53. F. Vollmer, S. Arnold, "Whispering gallery mode biosensing: label-free detection down to single molecules", Nature Methods, Vol. 5, No. 7, July 2008.

54. Zvyagin, A. V.; Goto, K., Optical Society of America 1998, 15, 3003. 
55. D. W. Vernooy, V. S. Ilchenko, ${ }^{*}$ H. Mabuchi, E. W. Streed, and H. J. Kimble, HighQ measurements of fused-silica microspheres in the near infrared, February 15, 1998 / Vol. 23, No. 4 / OPTICS LETTERS.

56. Wei Liang, Yong Xu, Yanyi Huang, and Amnon Yariv, J. G. Fleming, and ShawnYu Lin, Mie scattering analysis of spherical Bragg "onion" resonators, 2004 Optical Society of America.

57. MARK KUZNETSOV AND HERMANN A. HAUS, Radiation Loss in Dielectric Waveguide Structures by the Volume Current Method, IEEE JOURNAL OF QUANTUM ELECTRONICS, VOL. QE-19, NO. 10, OCTOBER 1983.

58. M. L. Gorodetsky, A. A. Savchenkov, and V. S. Ilchenko, Ultimate Q of optical microsphere resonators, April 1, 1996 / Vol. 21, No. 7 / OPTICS LETTERS.

59. G. Kozyreff, J. L. D. Juarez, J. Martorell, "Whispering gallery mode phase matching for surface second order nonlinear optical processes in spherical microresonatots", Apr 2008, Physical Review A, 77, 043817.

60. J. D. Jackson, Classical Electrodynamics, 3rd ed. (Wiley, NewYork, 1999).

61. M. Vanhoutte, "Biosensors based on circular resonators with vertical out coupling structures", Master's thesis, Ghent University, 2007.

62. R. P. Wang and M. M. Dumitrescu, "Theory of Optical Modes in Semiconductor Microdisk Lasers," J Appl. Phys., vol. 81, pp. 3391-3397, 1997.

63. K. Zhang and D. Li, Electromagnetic Theory for Microwaves and Optoelectronics. Berlin: Springer, 1998.

64. http://agamemnon.cord.org/cm/leot/course01_mod07/mod01-07frame.htm

65. Kawano, K., Kitoh, T., Introduction to Optical Waveguide Analysis: Solving Maxwell's Equation and the Schrodinger Equation, Wiley-Interscience; 1 edition (July 20, 2001)

66. Marcuse, D., Integrated optics, IEEE Press; (1973)

67. K. S. Yee, "Numerical Solution of Initial Boundary Value Problems Involving Maxwell's Equations in Isotropic Media," Antennas and Propagation, IEEE Transactions on, vol. 14, pp. 302-307, 1966. 
68. A. Bondeson, T. Rylander, and P. Ingelstr“om, Computational Electromagnetics. New York: Springer, 2005, pp. 57-86.

69. "Meep," Nanostructures and Computation Wiki, http://abinitio.mit.edu/wiki/index.php/Meep. Dec 18, 2008.

70. A. Taflove and S. C. Hagness, Computational Electrodynamics: The FiniteDifference Time-Domain Method. Boston: Artech House, 2000.

71. OptiBPM 8.0, Optiwave Systems Inc., Ottawa, ON, Canada, (www.optiwave.com).

72. "Coupling coefficient in strongly coupled dielectric waveguides", Yasuharu Suematsu and Katsumi Kishino, Radio Science, Volume 12, Number 4, pages 587592, July-August 1977.

73. "Integrated-optical directional coupler biosensor", B. J. Luff, R. D. Harris, J. S. Wilkinson, R. Wilson and D. J. Schiffrin, Opt. Lett. 21, 618-620 (1996).

74. P. H. Paul and G. Kychakoff, "Fiber-optic evanescent field absorption sensor,' 'Appl. Phys. Lett., vol. 51, pp. 12-14 1, 1987.

75. S. Simhony, I. Schnitzer, A. Katzir, and E. M. Kosower, "Evanescent wave infrared spectroscopy of liquids using silver halide optical fibers,' J. Appl. Phys., vol. 64, pp. 3732-3734, 1988.

76. J. R. Nightingale, R. Goswami, J. Duperre, J. M. Dawson, L. A. Hornak, and D. Korakakis, "Use of IBAD and low temperature annealing for the fabrication of low loss, vertically stacked alumina waveguide structures," J. Vac. Sci. Technol. B, vol. 26, no. 5, Sep/Oct 2008.

77. P. Poloju, P. Samudrala, J. R. Nightingale, D. Korakakis, L. A. Hornak, "Characterization of Alumina Optical Waveguides Grown by Ion Beam Assisted Deposition for SPARROW Biosensors," MRS Conference Proceedings, Fall 2006.

78. P. Martin, R. P. Netterfield, and W. G. Sainty, "Modification of the Optical and Structural Properties of Dielectric $\mathrm{ZrO}_{2}$ Films by Ion-Assisted Deposition," J. Appl. Phys., vol. 55, issue 1, pp. 235-241, Jan. 1984).

79. X. Fan, I. M. White, H. Zhu, J. D. Suter, and H. Oveys, "Overview of novel integrated optical ring resonator bio/chemical sensors," Proc. SPIE, vol. 6452, 2007. 
80. H. A. Al-Abadleh, V. H. Grassian "FT-IR Study of Water Adsorption on Aluminum Oxide Surfaces," Langmuir, vol. 19, pp. 341-347, 2003.

81. K. Singh, S. Mohan, "Kinetic studies of the sucrose adsorption onto an alumina interface," Applied Surface Science, vol. 221, pp. 308-318, 2004.

82. Y. Wang, L. Lin, B.S. Zhu, Y.X. Zhu, Y.C. Xie, "Different dispersion behavior of glucose and sucrose on alumina and silica surfaces," Applied Surface Science, vol. 254, pp. 6560-6567, 2008.

83. R. Goswami, J. R. Nightingale, J. A. Duperre III, M. S. Lim, J. M. Dawson, A. Timperman, D. Korakakis and L. A. Hornak, "Surface Loading Sensitivity Characterization of a Resonant Planar Optical Waveguide Stack", IEEE Photonics Technology Letters, March 2012.

84. Lee Rodak, "Surfactants and Digital Alloys for Strain Relief in III-Nitride Distributed Bragg Reflectors and Related Heterostructures via Metal Organic Vapor Phase Epitaxy", PhD Dissertation, West Virginia University, 2011.

85. Operation manual, SEM-JSM-7600F Brochure, JEOL USA.

86. http://www.ece.tufts.edu/ hopwood/lab/plasmaetching.htm

87. J. P. Berenger, J. Comput. Phys. 114, 185 (1994).

88. K. Lee , A. Kadiyala, L. E. Rodak, R. Goswami, V. Kumbham, B. A. Bearce, J. Justice, J. Peacock, J. M. Dawson, L. A. Hornak, and D. Korakakis, "Enhanced Emission from $\operatorname{In}_{x} \mathrm{Ga}_{1-x} \mathrm{~N}$-based LED Structures Using III-Nitride based Distributed Bragg Reflector”, Materials Research Society Conference, 2011

89. V. S. Ilchenko and L. Maleki, "Novel Whispering-Gallery Resonators for Lasers, Modulators, and Sensors," Proc. SPIE, vol. 4270, pp. 120-130, 2001. 